\title{
High-Affinity Alkynyl Bisubstrate Inhibitors of Nicotinamide N-Methyltransferase (NNMT)
}

\section{Supporting Information Part 2: NMR Spectra}

\author{
Rocco L. Policarpo*, Ludovic Decultot*, Elizabeth May ${ }^{\dagger}$, Petr Kuzmič ${ }^{\S}$, Samuel Carlson ${ }^{\dagger}$, \\ Danny Huang*, Vincent Chu*, Brandon Wright*, Saravanakumar Dhakshinamoorthy ${ }^{\ddagger}$, \\ Aimo Kannt*, Shilpa Rani ${ }^{\ddagger}$, Sreekanth Dittakavi ${ }^{\ddagger}$, Joseph Panarese*, Rachelle Gaudet ${ }^{\dagger}$, \\ and Matthew D. Shair*
}

* Department of Chemistry \& Chemical Biology, Harvard University, Cambridge MA 02138, USA

${ }^{\dagger}$ Department of Molecular \& Cellular Biology, Harvard University, Cambridge MA 02138, USA

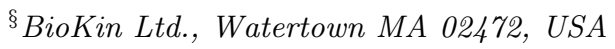

${ }^{\ddagger}$ Jubilant Biosys Ltd., Yeshwantpur Bangalore - 560 022, Karnataka, India

${ }^{\star}$ Sanofi Research and Development, Industriepark Hoechst, H823, D-65926, Frankfurt am Main, Germany 


\section{List of NMR Spectra}

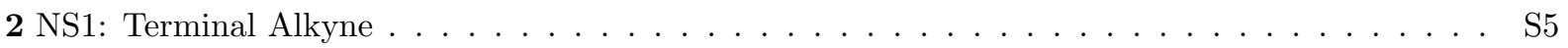

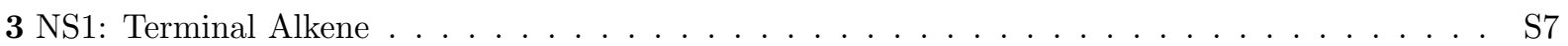

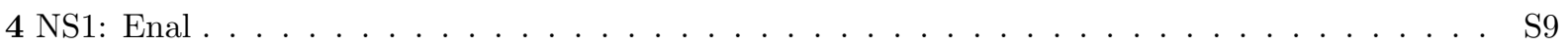

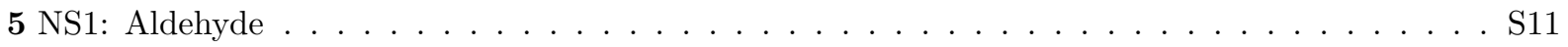

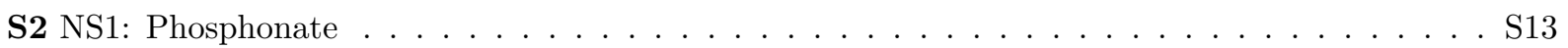

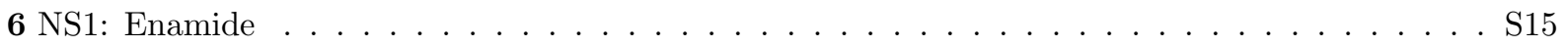

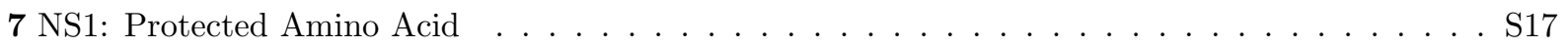

S3 TIPS Cleavage Product . . . . . . . . . . . . . . . . . . S19

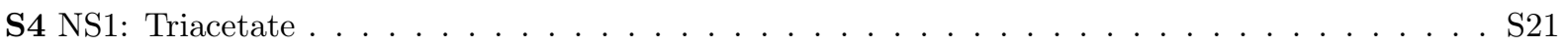

8 NS1: Nucleoside . . . . . . . . . . . . . . . . . . . . . . . . . S S23

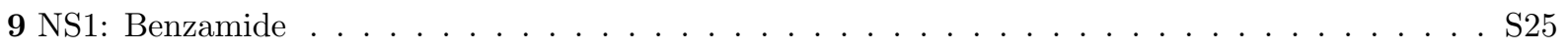

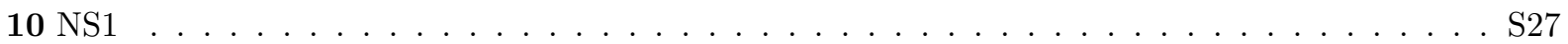

S6 Desthia-SAH: Methyl Ester . . . . . . . . . . . . . . . . . . . . . . S29

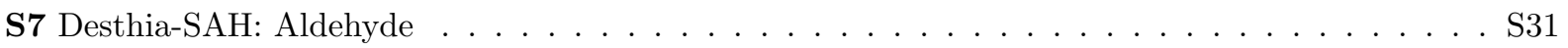

S8 Desthia-SAH: Enamide . . . . . . . . . . . . . . . . . . . . . S33

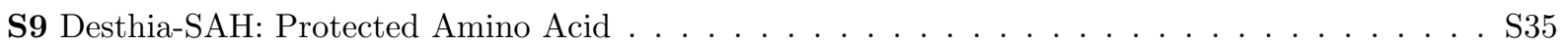

S10 Desthia-SAH: Triacetate . . . . . . . . . . . . . . . . . . S37

S11 Desthia-SAH: Nucleoside . . . . . . . . . . . . . . . . . . . . . . . S39

11 Desthia-SAH . . . . . . . . . . . . . . . . . . . . . . S41

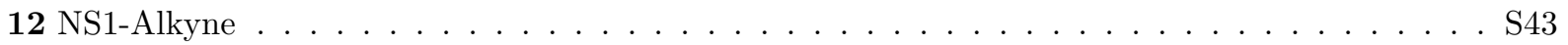

S12 NS1-Phenyl: Benzamide . . . . . . . . . . . . . . . . . S45

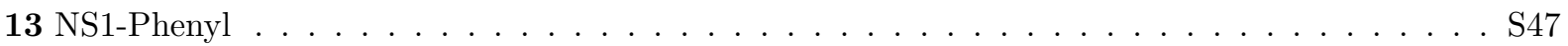

S13 NS1-6'Epi: Aldehyde . . . . . . . . . . . . . . . . . . . S49

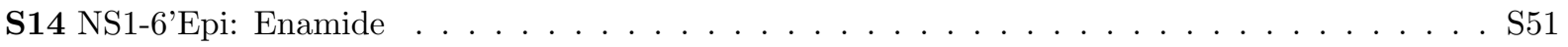

S15 NS1-6'Epi: Protected Amino Acid . . . . . . . . . . . . . . . . . S53

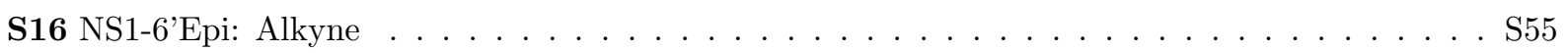

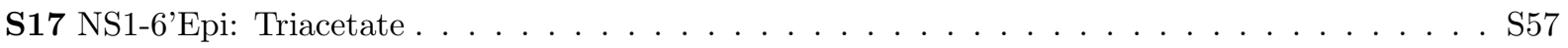

S18 NS1-6'Epi: Nucleoside . . . . . . . . . . . . . . . . . . . . . . . S59

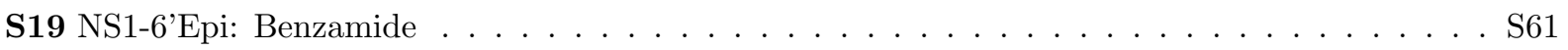

14 NS1-6'Epi . . . . . . . . . . . . . . . . . . . . . . . S63

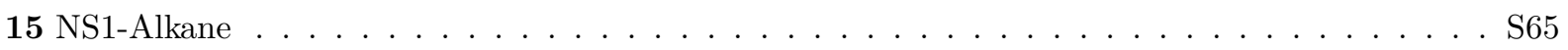

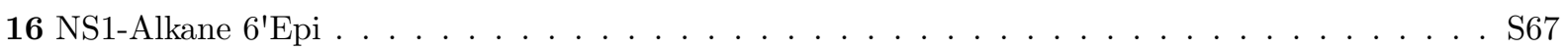

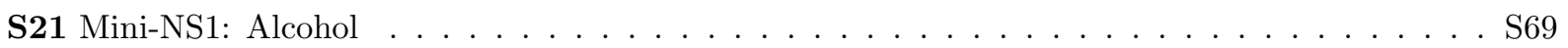

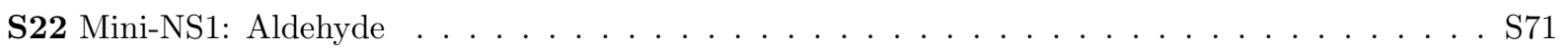

S23 Mini-NS1: Alkyne . . . . . . . . . . . . . . . . . . . . . S S73

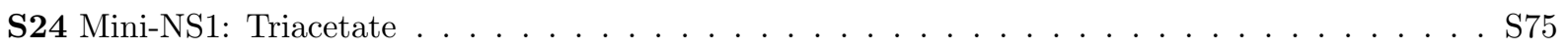

S25 Mini-NS1: Nucleoside . . . . . . . . . . . . . . . . . . . . . . S77

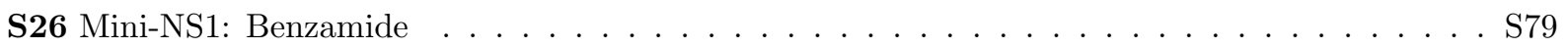

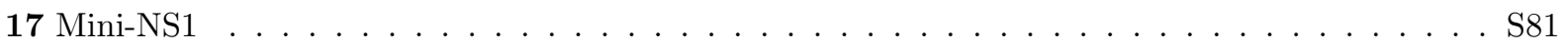

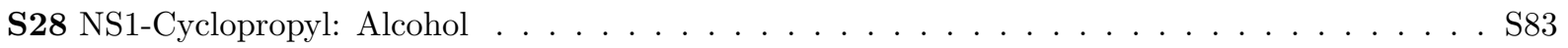

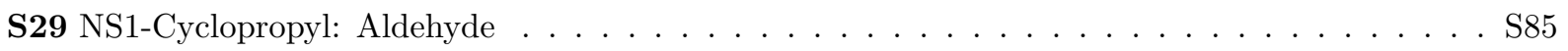

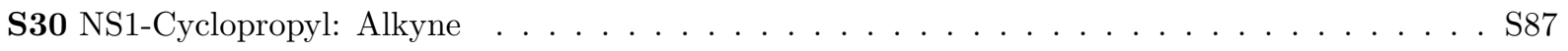


S31 NS1-Cyclopropyl: Triacetate . . . . . . . . . . . . . . . . . . S89

S32 NS1-Cyclopropyl: Nucleoside . . . . . . . . . . . . . . . . . . . . . . . S91

S33 NS1-Cyclopropyl: Benzamide . . . . . . . . . . . . . . . . . S93

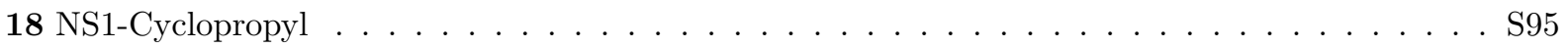

S34 NS1-Desadenine: Benzamide . . . . . . . . . . . . . . . . . . . S97

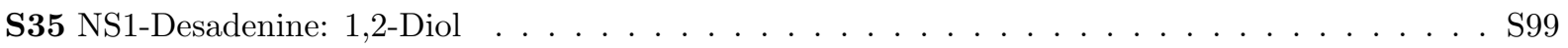

19 NS1-Desadenine . . . . . . . . . . . . . . . . . . . . . . . . . . S101

S36 NS1-Carboxylic Acid: Enoate . . . . . . . . . . . . . . . . . . . S103

S37 NS1-Carboxylic Acid: Methyl Ester . . . . . . . . . . . . . . . . . . S105

S38 NS1-Carboxylic Acid: Alkyne . . . . . . . . . . . . . . . . . . . . S107

S39 NS1-Carboxylic Acid: Triacetate . . . . . . . . . . . . . . . . . . . S109

S40 NS1-Carboxylic Acid: Nucleoside . . . . . . . . . . . . . . . . . . . . . S111

S41 NS1-Carboxylic Acid: Benzamide . . . . . . . . . . . . . . . . . S113

20 NS1-Carboxylic Acid . . . . . . . . . . . . . . . . . . . . S115

S42 NS1-Amine: Nitro-olefin . . . . . . . . . . . . . . . . . . . . . . S117

S43 NS1-Amine: Trifluoroacetamide . . . . . . . . . . . . . . . . . . . . . . S119

S44 NS1-Amine: Alkyne . . . . . . . . . . . . . . . . . . . . . . . S121

S45 NS1-Amine: Triacetate . . . . . . . . . . . . . . . . . . . . . S123

S46 NS1-Amine: Nucleoside . . . . . . . . . . . . . . . . . . . . . . . S125

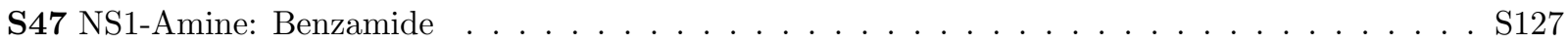

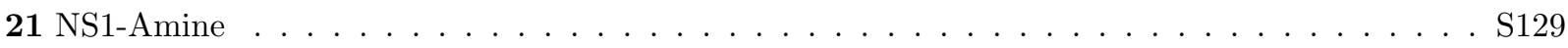

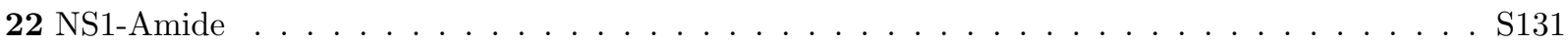

S48 NS1-MethylEster: Carbamate . . . . . . . . . . . . . . . . . . . . S133

S49 NS1-MethylEster: Triacetate . . . . . . . . . . . . . . . . . . . S135

S50 NS1-MethylEster: Nucleoside . . . . . . . . . . . . . . . . . . . . . S137

S51 NS1-MethylEster: Benzamide . . . . . . . . . . . . . . . . . . . S139

23 NS1-MethylEster . . . . . . . . . . . . . . . . . . . . . . . S141

24 NS1-AminoAmide . . . . . . . . . . . . . . . . . . . . . . . S143

S52 NS1-Urea: TIPS alkyne Alcohol . . . . . . . . . . . . . . . . . . . S145

S53 NS1-Urea: Alkynyl Alcohol _ . . . . . . . . . . . . . . . . . . . . . . . S147

S54 NS1-Urea: Nosyl Amine . . . . . . . . . . . . . . . . . . . . . . . . . . S149

S55 NS1-Urea: Triacetate . . . . . . . . . . . . . . . . . . . . . S151

S56 NS1-Urea: Nucleoside . . . . . . . . . . . . . . . . . . . . . . . . S153

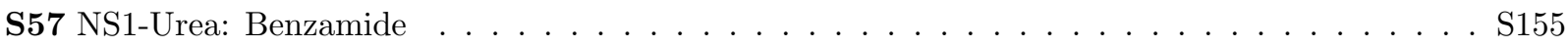

S58 NS1-Urea: Urea . . . . . . . . . . . . . . . . . . . . . . . . . . . . S157

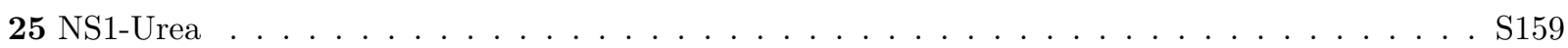

S59 Homo-NS1: Nitrile . . . . . . . . . . . . . . . . . . . . . . . . S161

S61 Homo-NS1: Enamide . . . . . . . . . . . . . . . . . . . . . . S163

S62 Homo-NS1: Protected Amino Acid . . . . . . . . . . . . . . . . . S165

S63 Homo-NS1: Alkyne . . . . . . . . . . . . . . . . . . . . . . . . S167

S64 Homo-NS1: Triacetate . . . . . . . . . . . . . . . . . . . . . S169

S65 Homo-NS1: Nucleoside . . . . . . . . . . . . . . . . . . . . . . . . S S171

S66 Homo-NS1: Benzamide . . . . . . . . . . . . . . . . . . . . . . . S173 


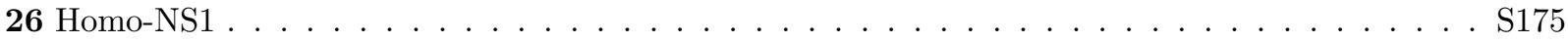

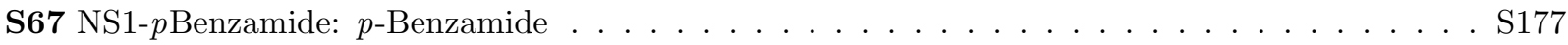

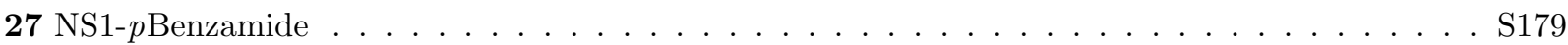

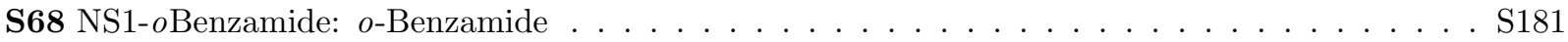

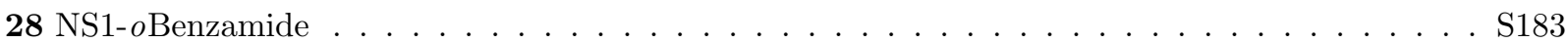

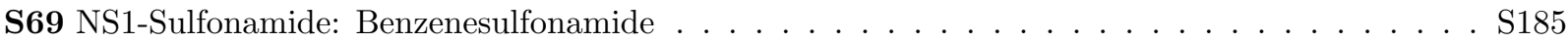

29 NS1-Sulfonamide . . . . . . . . . . . . . . . . . . . . . . . . . . . S187

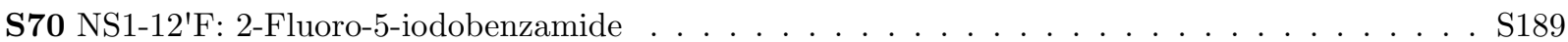

S71 NS1-12'F: Fluorobenzamide . . . . . . . . . . . . . . . . . . . S191

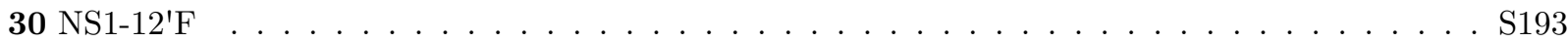

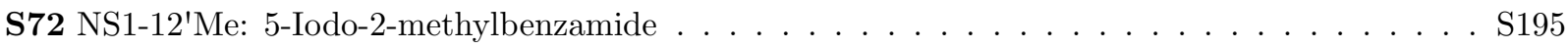

S73 NS1-12'Me: Methylbenzamide . . . . . . . . . . . . . . . . . . . . . . . S197

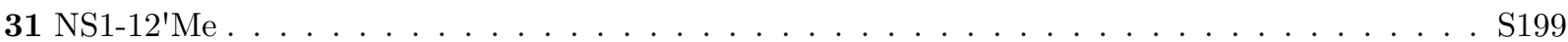

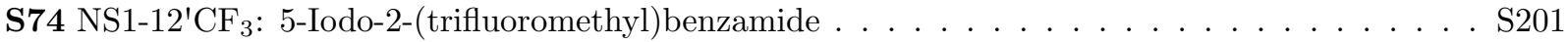

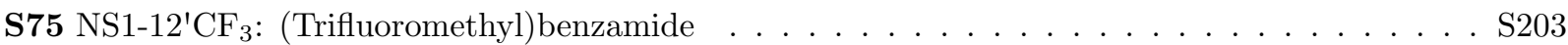

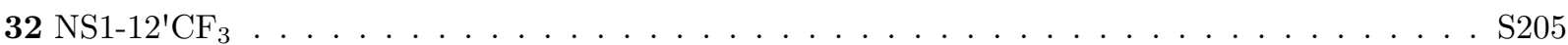

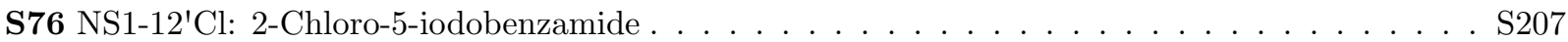

S77 NS1-12'Cl: Chlorobenzamide . . . . . . . . . . . . . . . . . . . . . S209

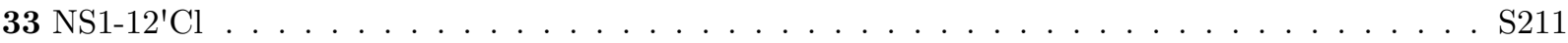

S78 NS1-Benzolactam6: Dihydro Isoquinolinone . . . . . . . . . . . . . . . . . . S213

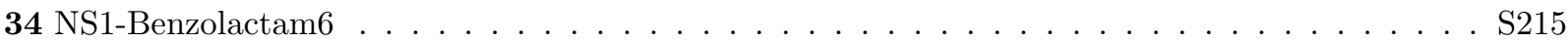

35 NS1-Benzolactam5 . . . . . . . . . . . . . . . . . . . . . . . . S217

S80 NS1-Methylenedioxy: Iodobenzamide . . . . . . . . . . . . . . . . . . . . . . . S219

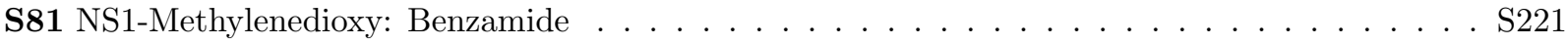

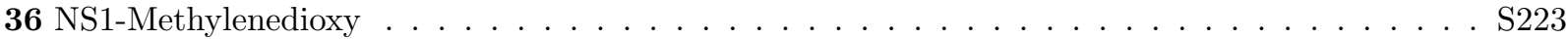

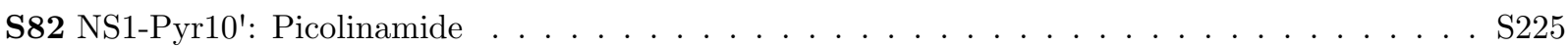

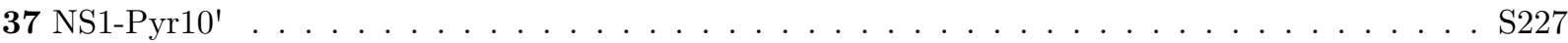

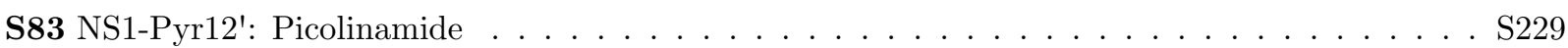

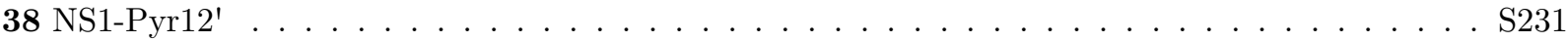

S84 NS1-Pyr13': Nicotinamide . . . . . . . . . . . . . . . . . . . . . S233

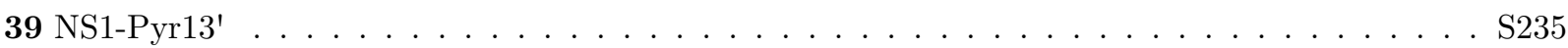

S85 NS1-Pyr14': Isonicotinamide . . . . . . . . . . . . . . . . . . . S237

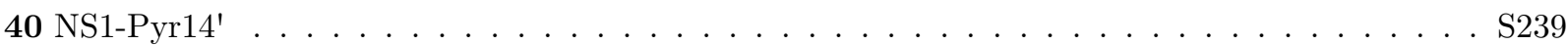

S86 NS1-Aminonaphthalene: Naphthalene . . . . . . . . . . . . . . . . . . . . . S241

41 NS1-Aminonaphthalene . . . . . . . . . . . . . . . . . . . . . . S243 


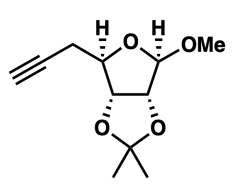

00
0
0
0
0
$i$

2, ${ }^{1} \mathrm{H}, 600 \mathrm{MHz}, \mathrm{CDCl}_{3}$

$\stackrel{G}{G}$

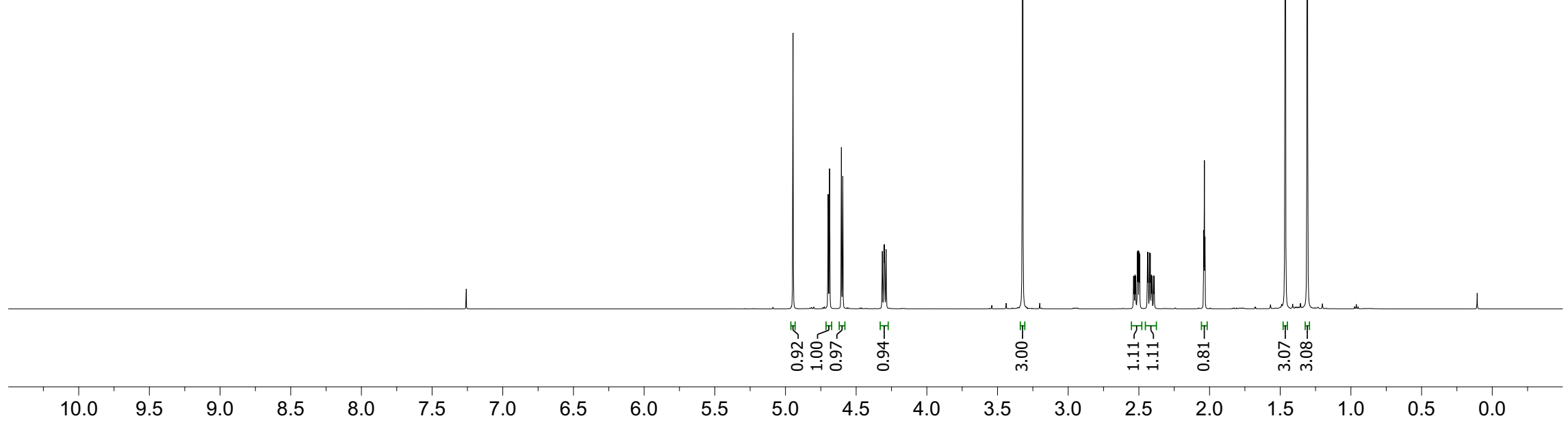




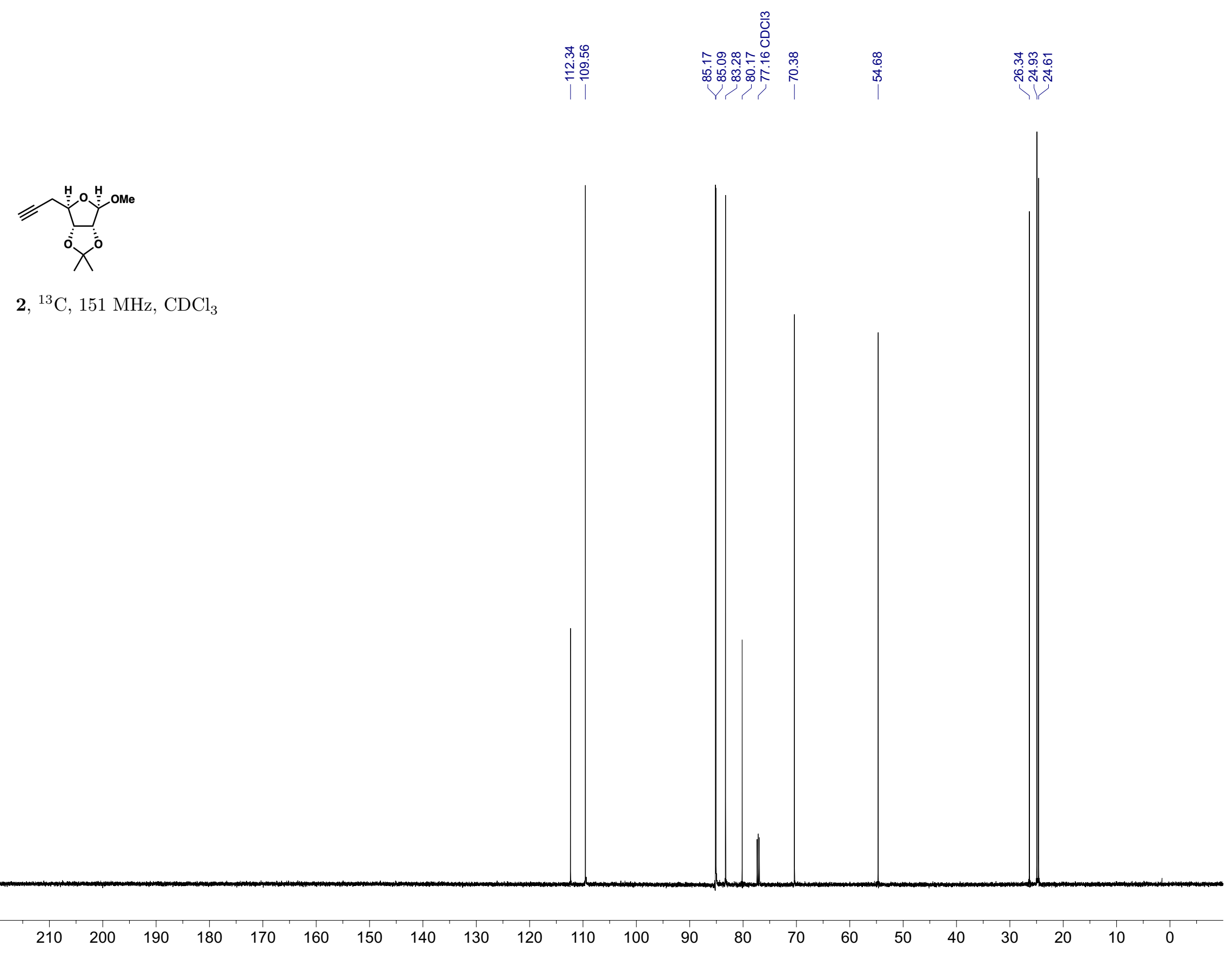




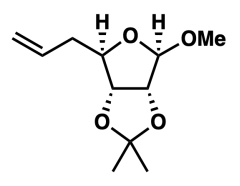

3, ${ }^{1} \mathrm{H}, 600 \mathrm{MHz}, \mathrm{CDCl}_{3}$

0
0
0
0
0
$i$

ปs

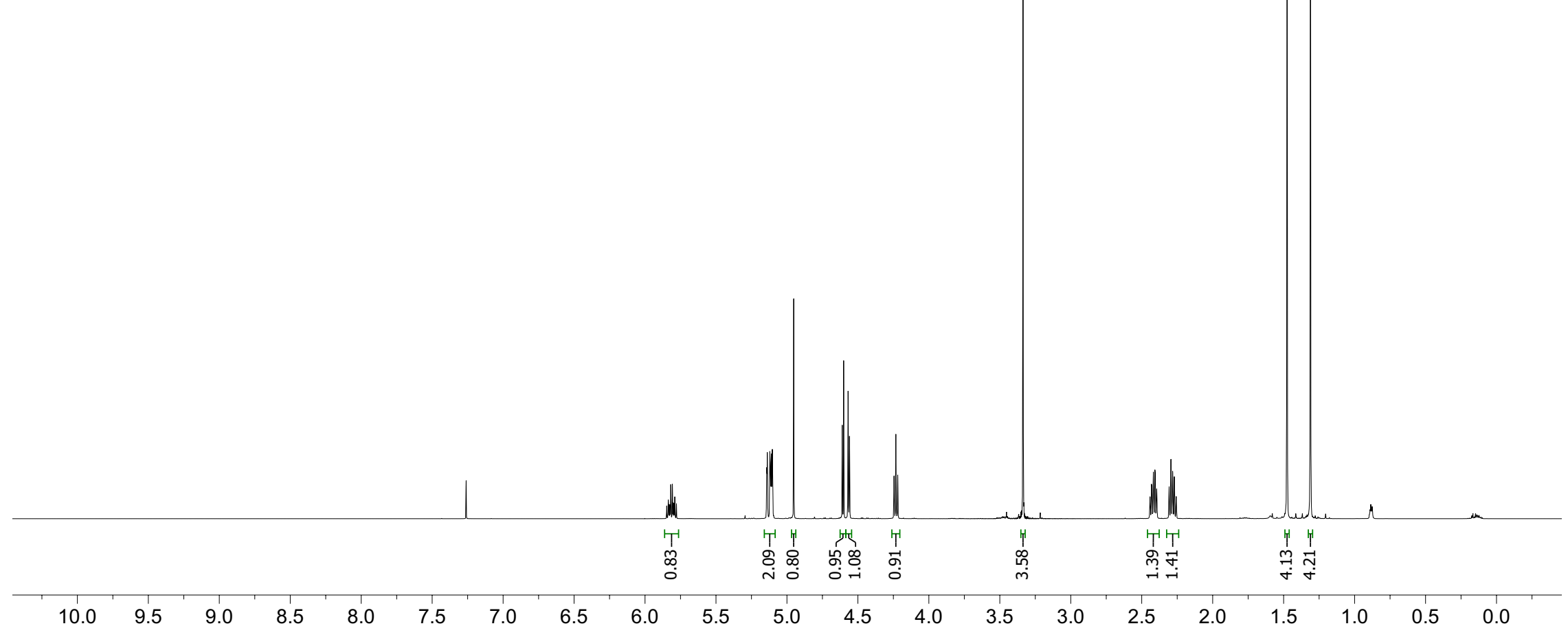




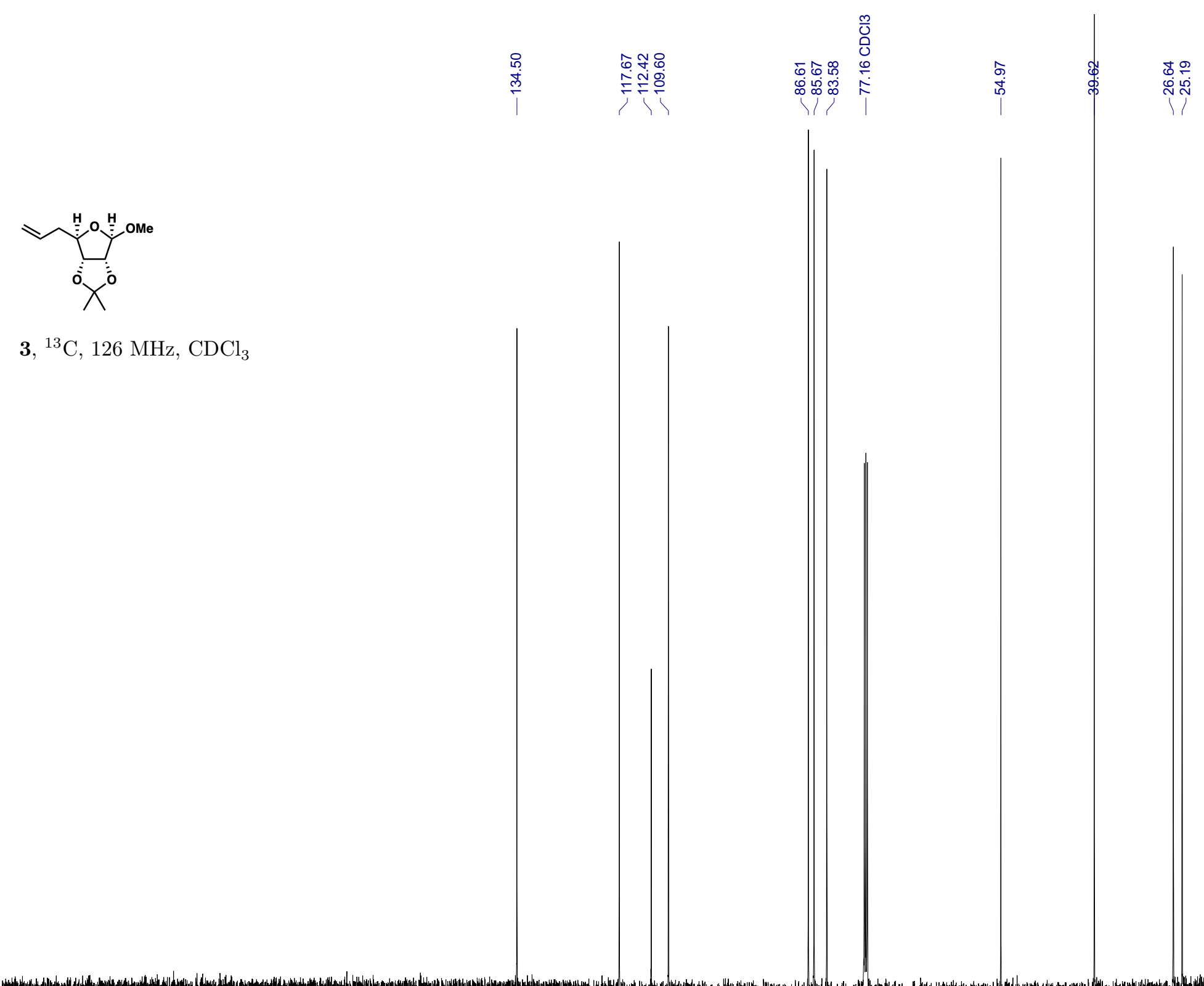

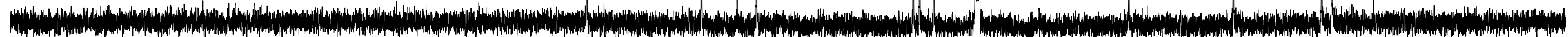




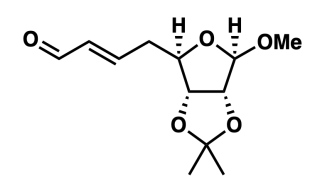

4, ${ }^{1} \mathrm{H}, 500 \mathrm{MHz}, \mathrm{CDCl}_{3}$

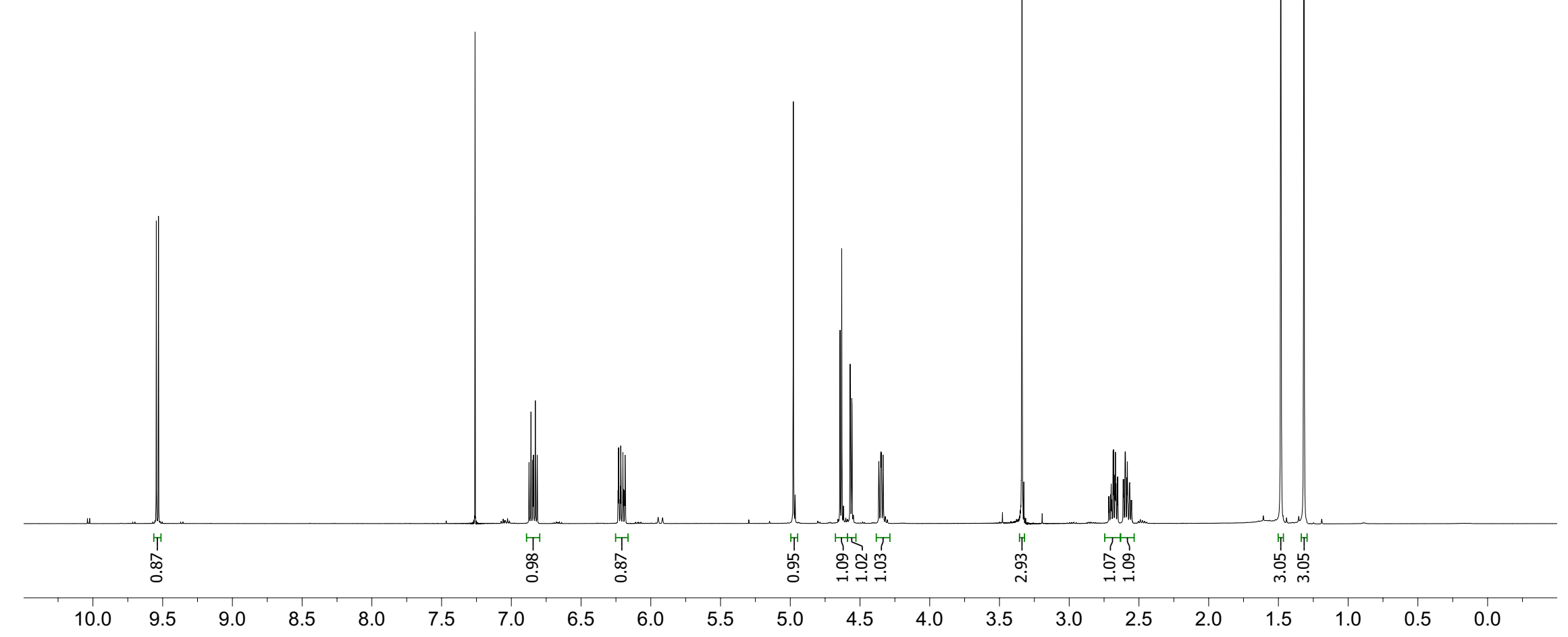




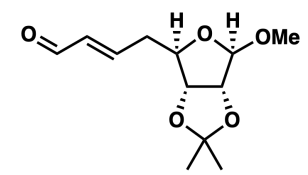

$4,{ }^{13} \mathrm{C}, 151 \mathrm{MHz}, \mathrm{CDCl}_{3}$

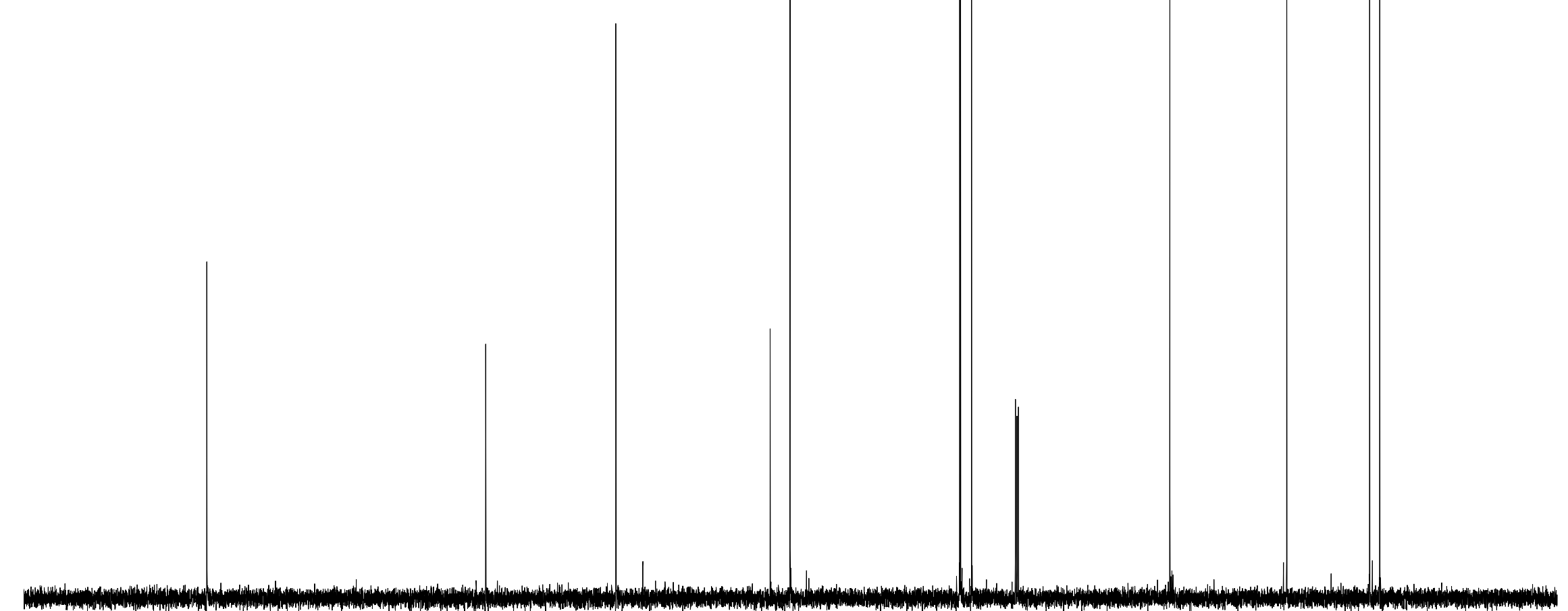




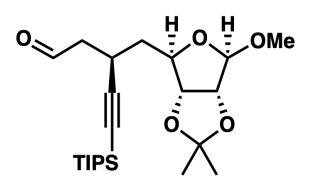

5, ${ }^{1} \mathrm{H}, 600 \mathrm{MHz}, \mathrm{CDCl}_{3}$

$\stackrel{\Xi}{\boxminus}$

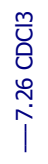

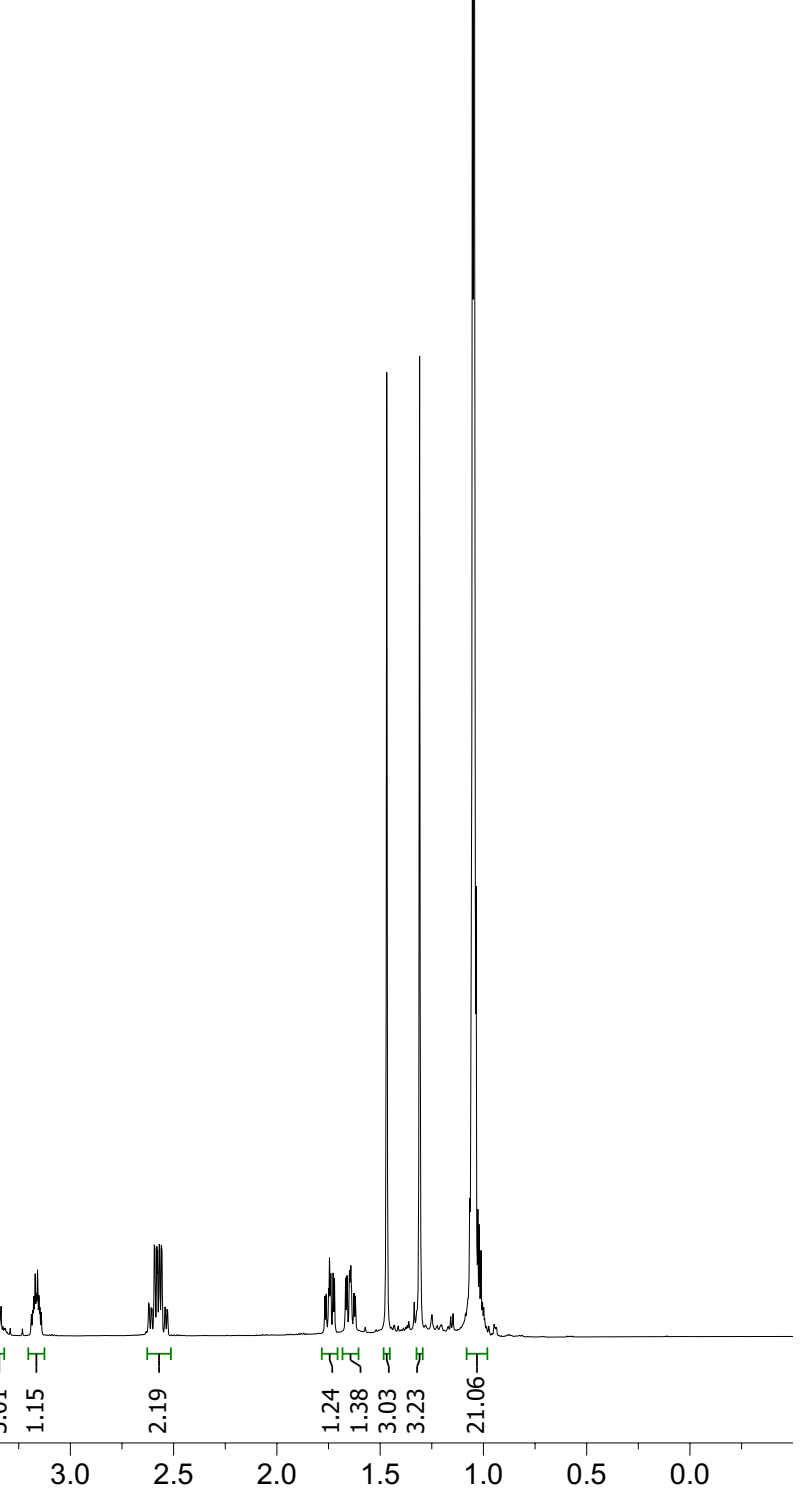




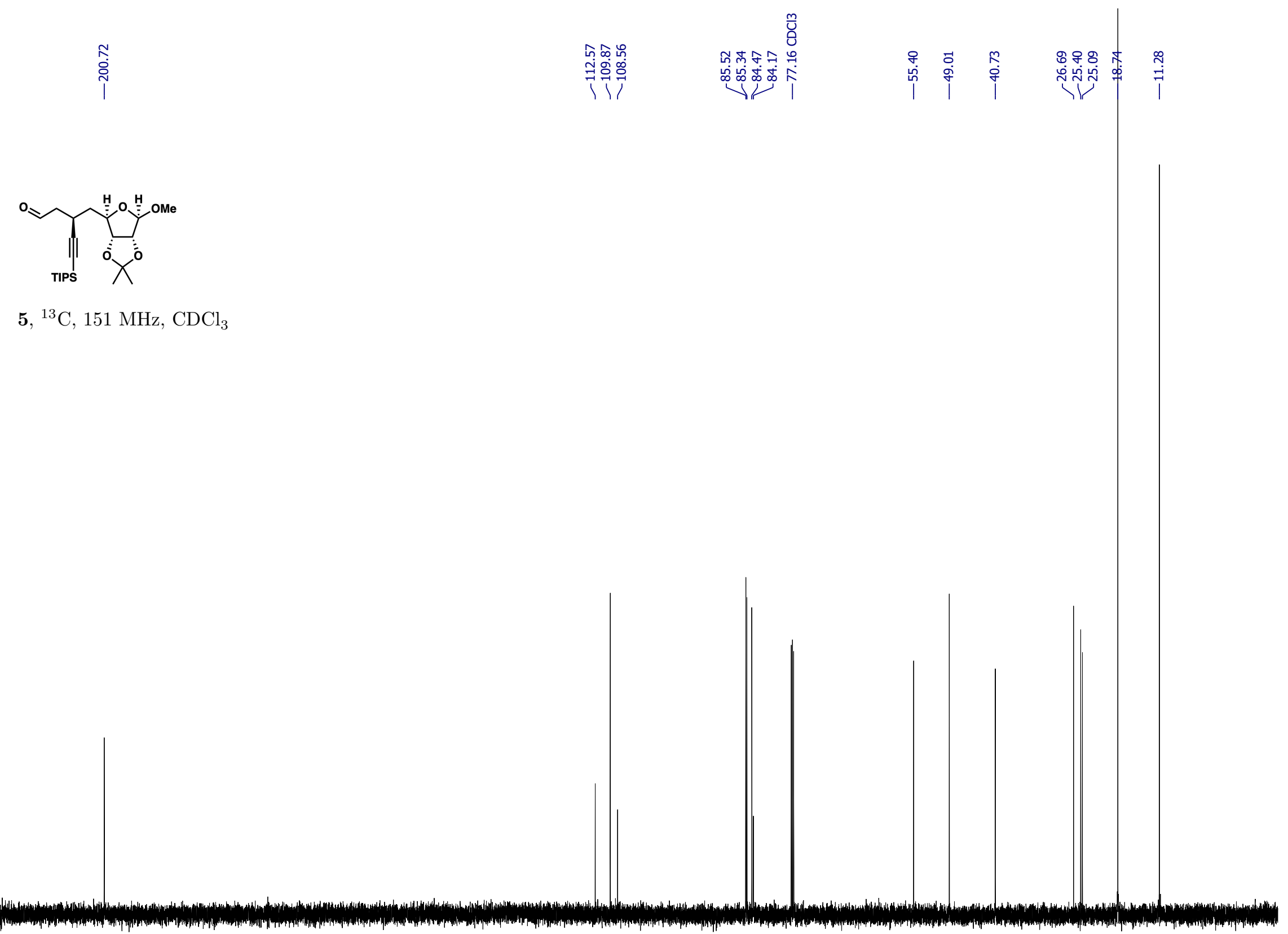

$210 \quad 200$

$\begin{array}{llll}190 & 180 & 170 & 160\end{array}$

$150 \quad 140$

$130 \quad 120$

$110 \quad 100 \quad 90$

80

70

$60 \quad 50$

$40 \quad 30$

20 


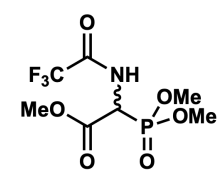

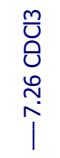

S2, ${ }^{1} \mathrm{H}, 600 \mathrm{MHz}, \mathrm{CDCl}_{3}$

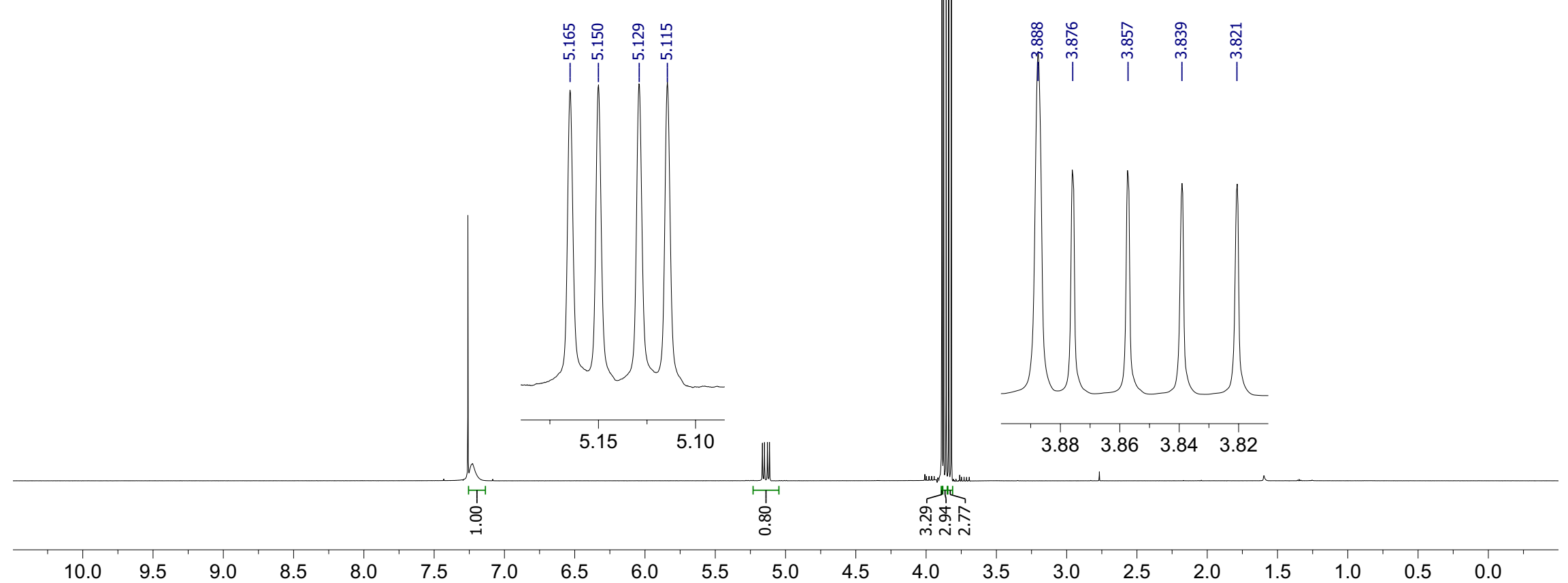


도ำ ํำ

นึ่

14

$\prod_{\mathrm{O}}^{\text {OMe }}$

S2, ${ }^{13} \mathrm{C}, 126 \mathrm{MHz}, \mathrm{CDCl}_{3}$ 


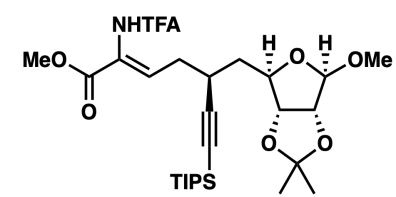

$\frac{m}{0}$
0
0
0

6, ${ }^{1} \mathrm{H}, 600 \mathrm{MHz}, \mathrm{CDCl}_{3}$

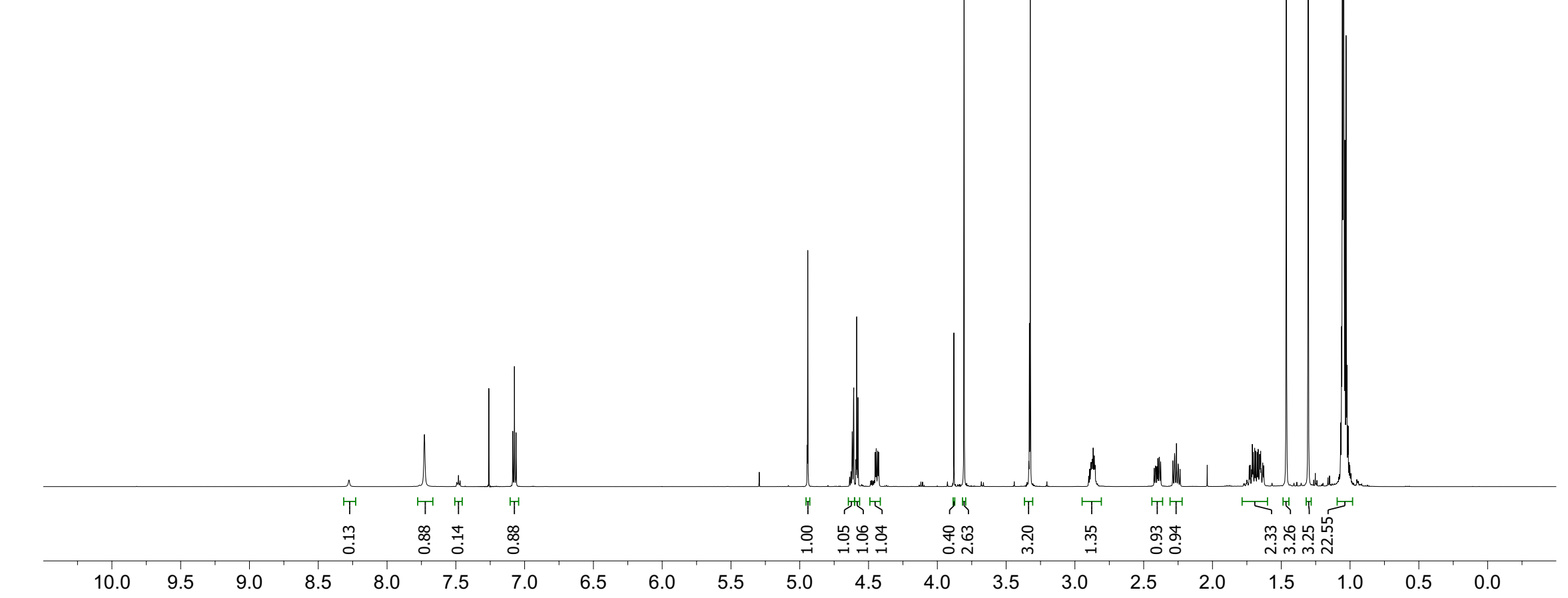




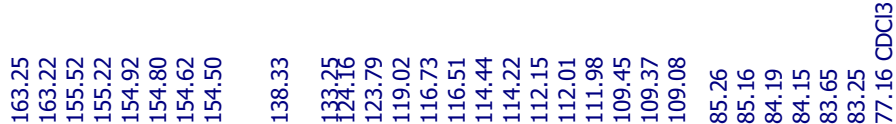

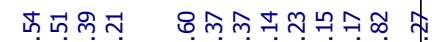

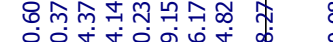

$\checkmark 1$

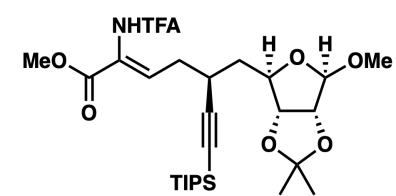

6, ${ }^{13} \mathrm{C}, 126 \mathrm{MHz}, \mathrm{CDCl}_{3}$

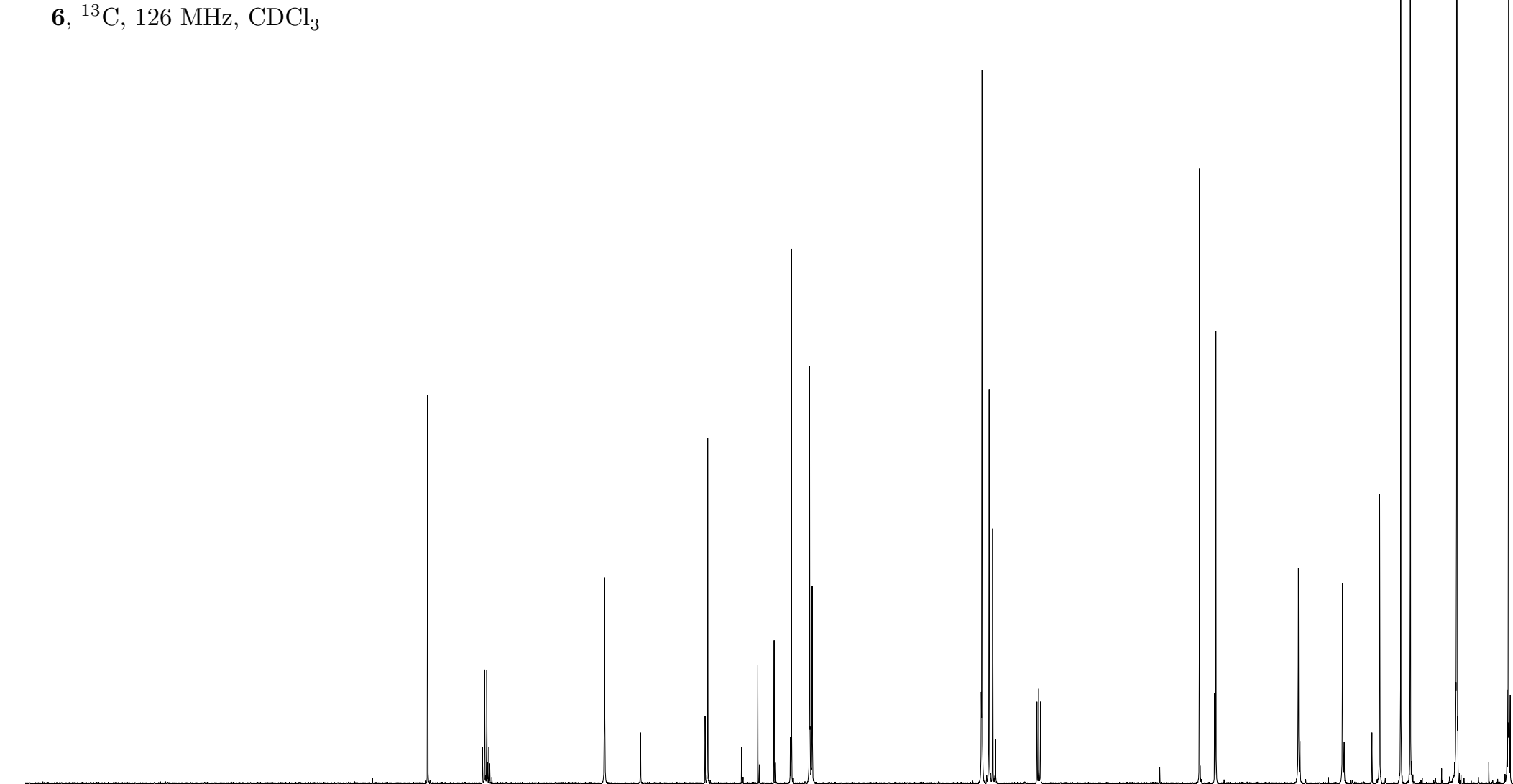

$\begin{array}{llll}190 & 180 & 170 \quad 160\end{array}$

150

$120 \quad 110$

80

70

$60 \quad 50$

$40 \quad 30$

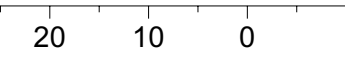




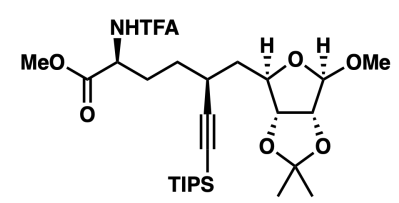

7, ${ }^{1} \mathrm{H}, 600 \mathrm{MHz}, \mathrm{CDCl}_{3}$

\section{.}




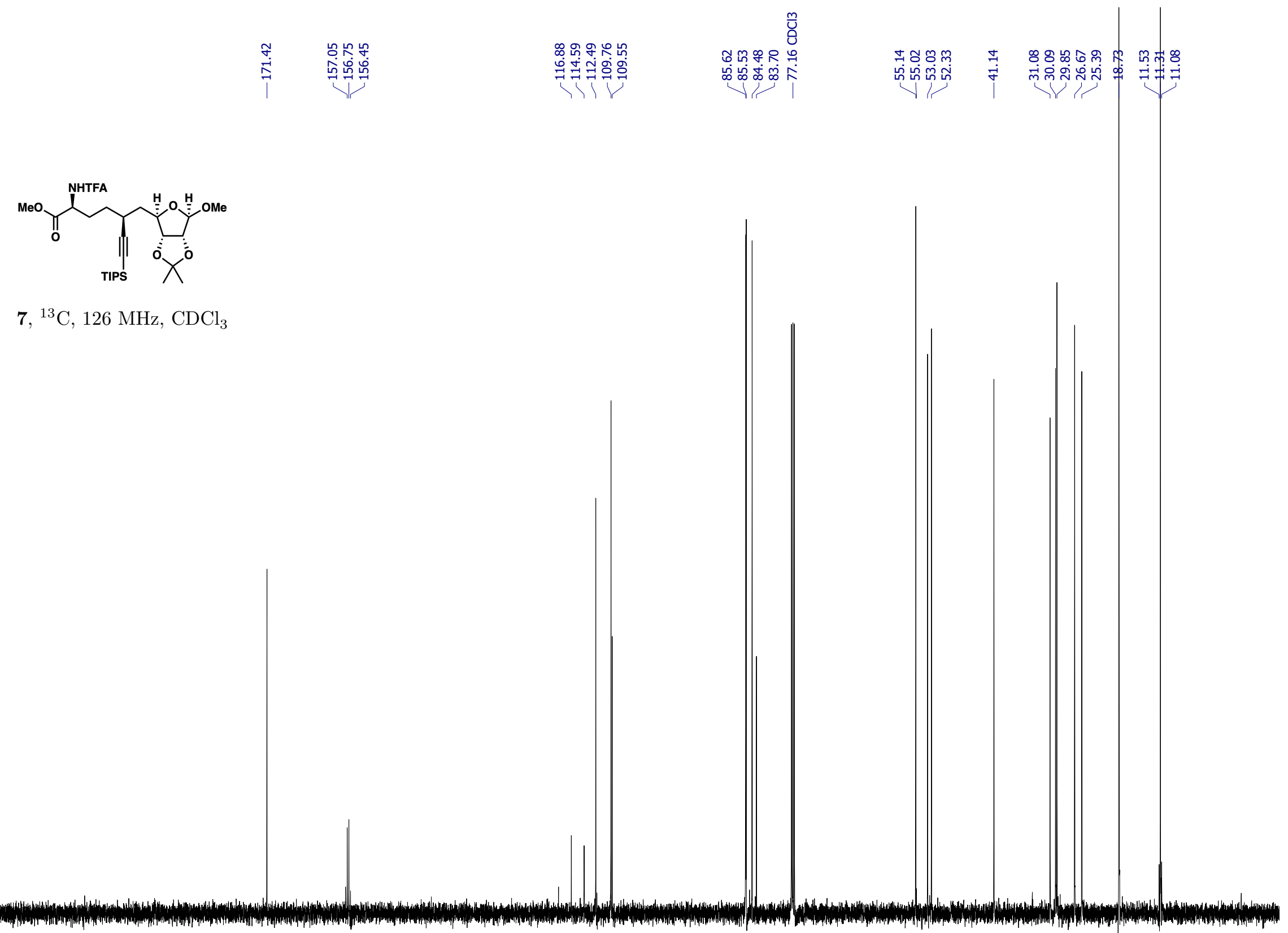

${ }^{3} \mathrm{C}, 126 \mathrm{M}$

$\begin{array}{llllll}210 & 200 & 190 & 180 & 170 & 160\end{array}$

$150 \quad 140$

$130 \quad 120$

$\begin{array}{lll}110 & 100 & 90\end{array}$

80

70

$60 \quad 50$

$40 \quad 30$

$20 \quad 10$ 


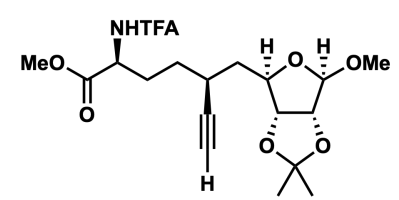

S3, ${ }^{1} \mathrm{H}, 600 \mathrm{MHz}, \mathrm{CDCl}_{3}$

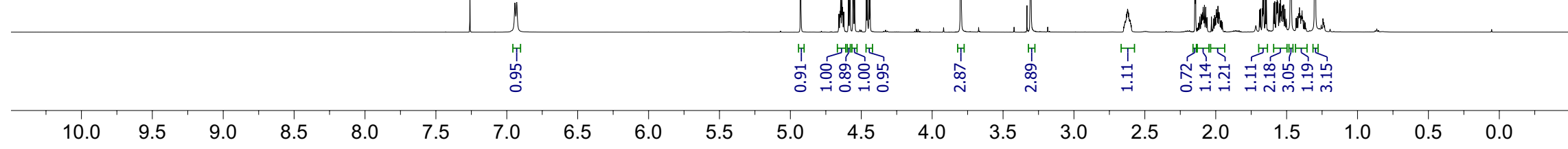




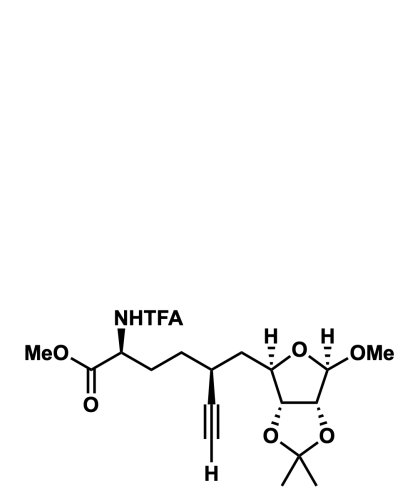

S3, ${ }^{13} \mathrm{C}, 126 \mathrm{MHz}, \mathrm{CDCl}_{3}$

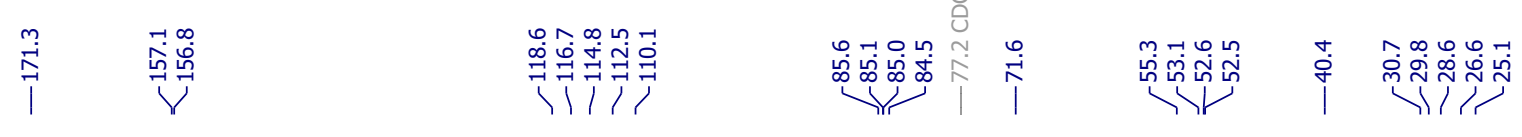

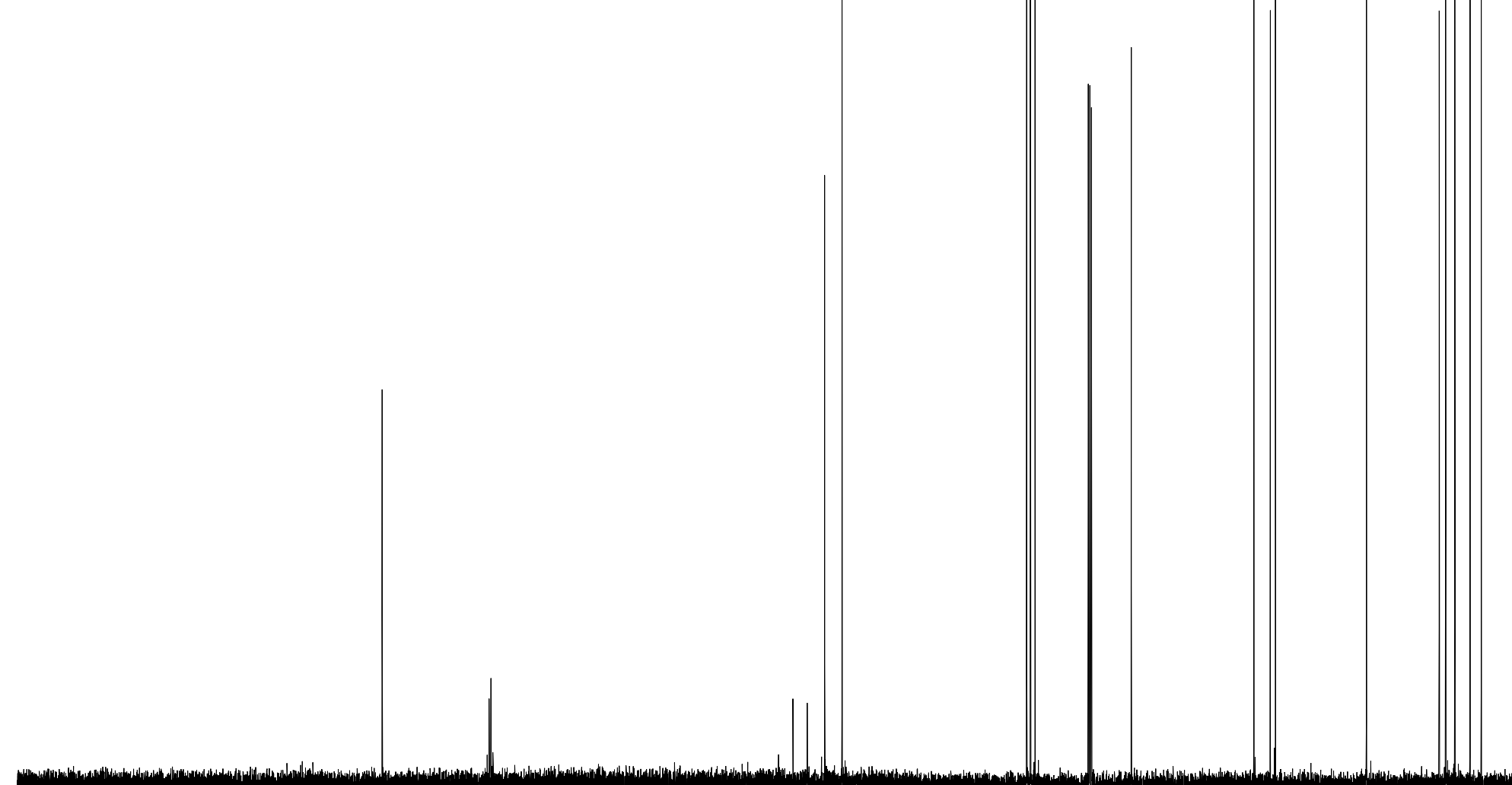

1. 


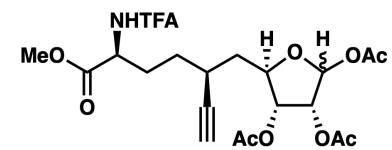

$\mathbf{S} 4,{ }^{1} \mathrm{H}, 600 \mathrm{MHz}, \mathrm{CDCl}_{3}$

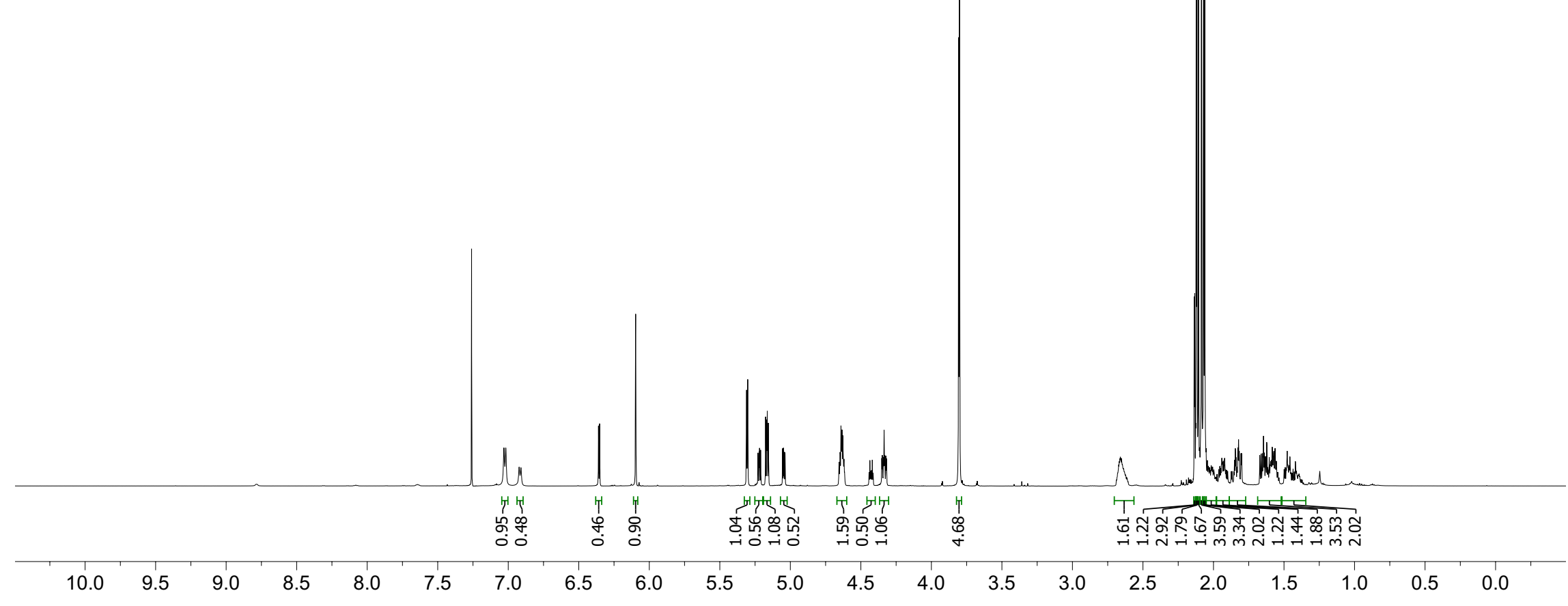




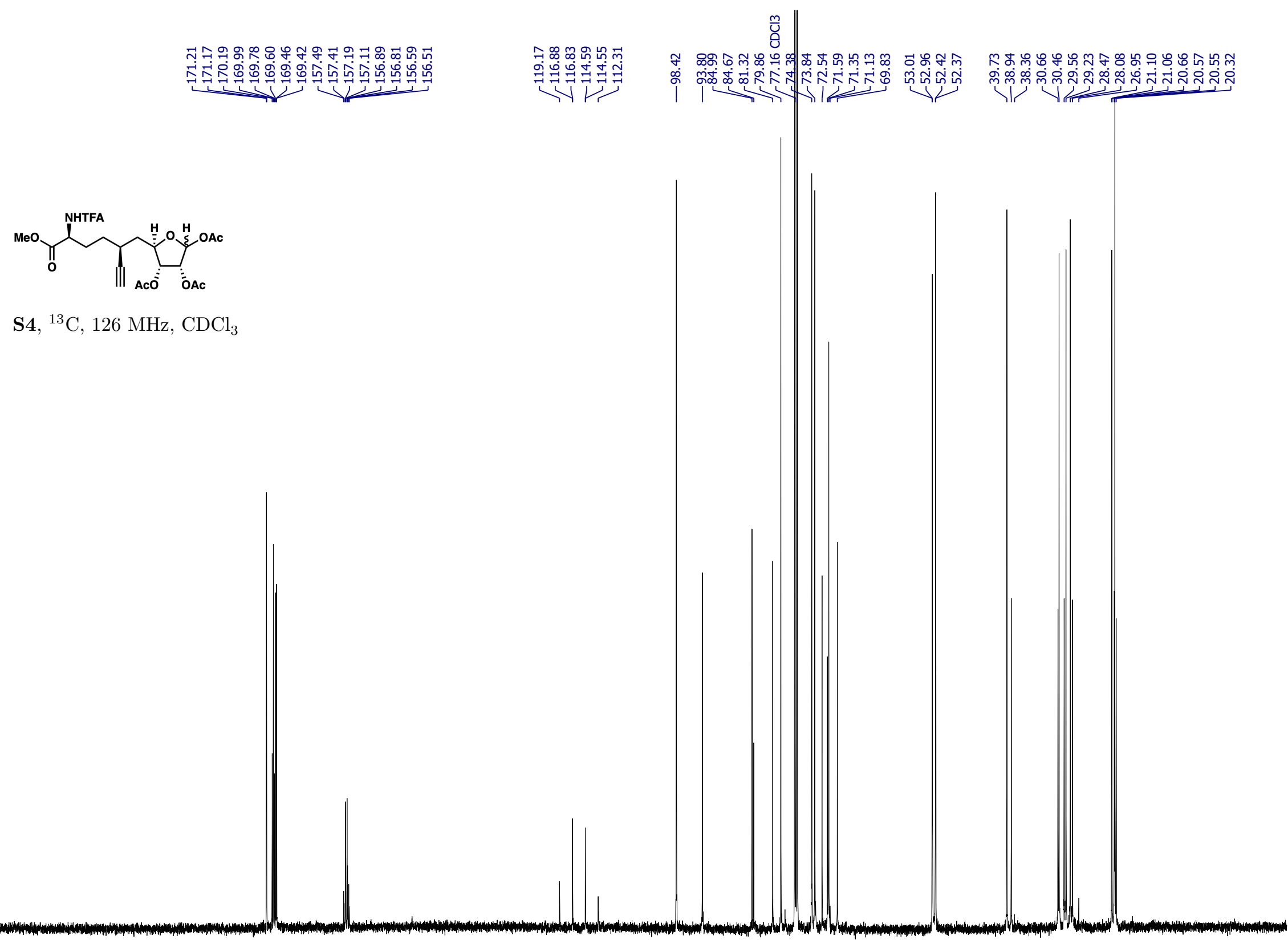

210200 190 180 $170 \quad 160$ 150 130 120 $110 \quad 100$ 90 $80 \quad 70$ 60 50 $40 \quad 30$ $20 \quad 10$ 0 


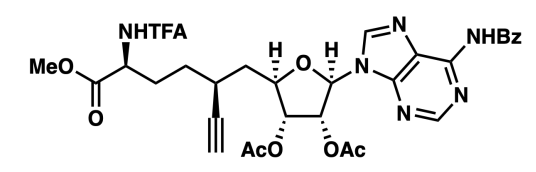

$8,{ }^{1} \mathrm{H}, 500 \mathrm{MHz}, \mathrm{CDCl}_{3}$

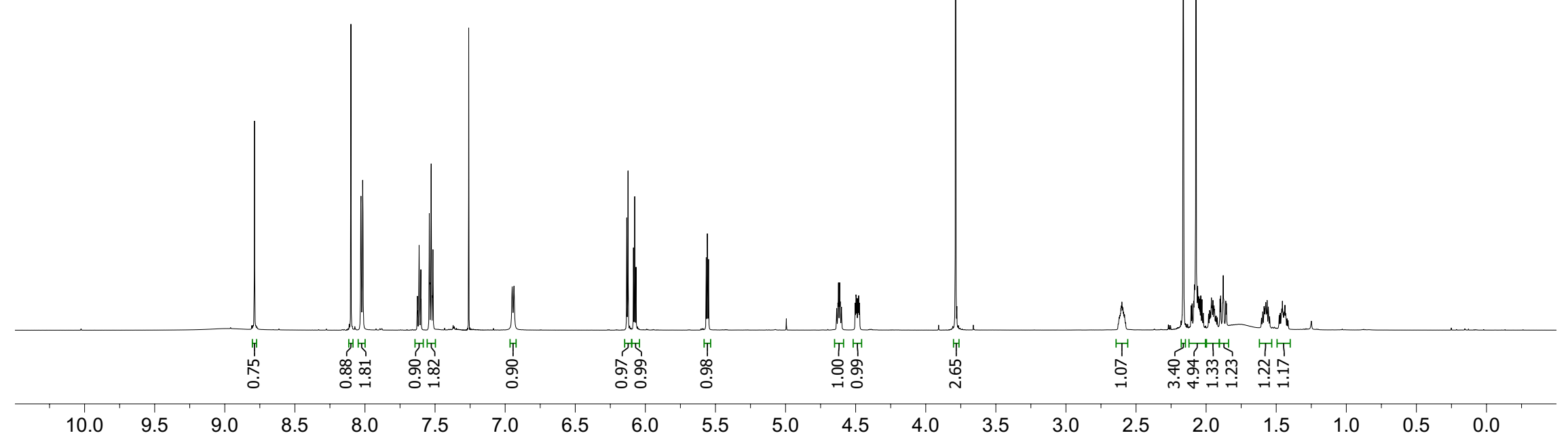




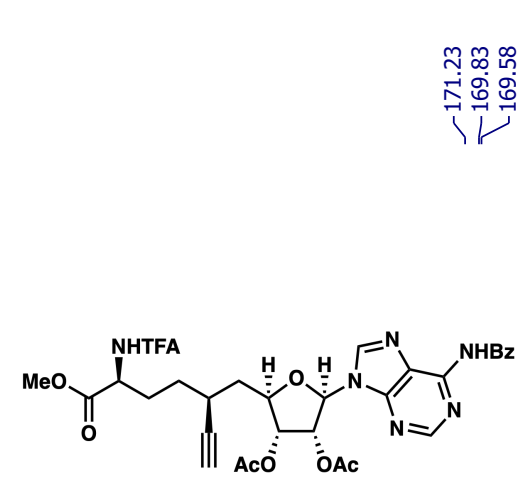

$\stackrel{0}{0}$

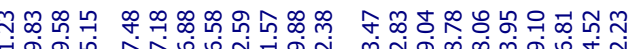

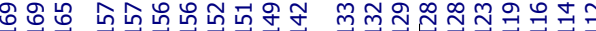

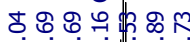

(1)

sin

त1 $1>1$

잉

री

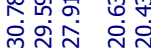

$8,{ }^{13} \mathrm{C}, 126 \mathrm{MHz}, \mathrm{CDCl}_{3}$ 


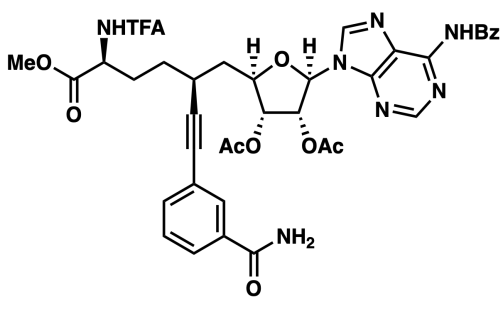

9, ${ }^{1} \mathrm{H}, 600 \mathrm{MHz}, \mathrm{CD}_{2} \mathrm{Cl}_{2}$

诧

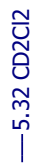

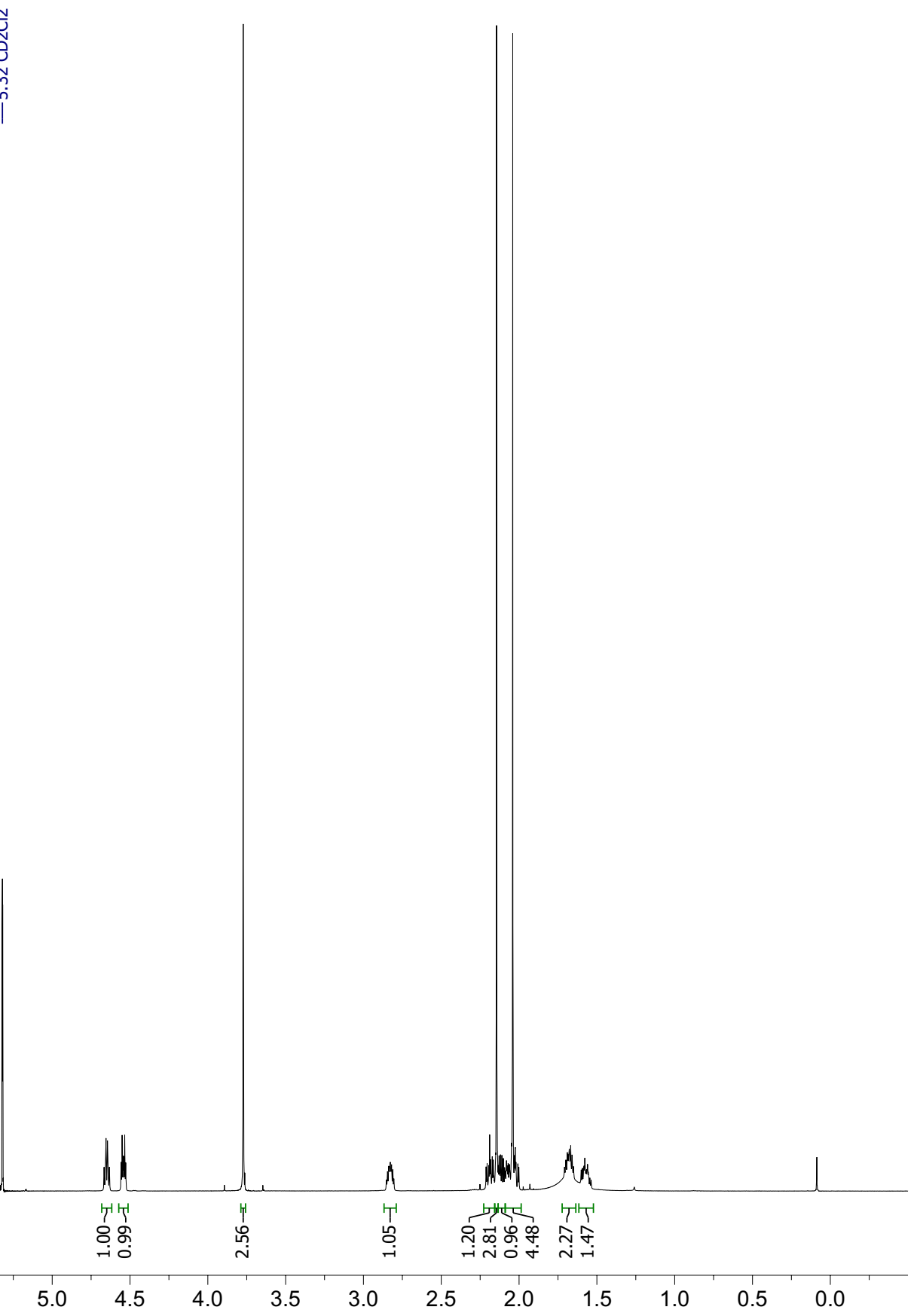




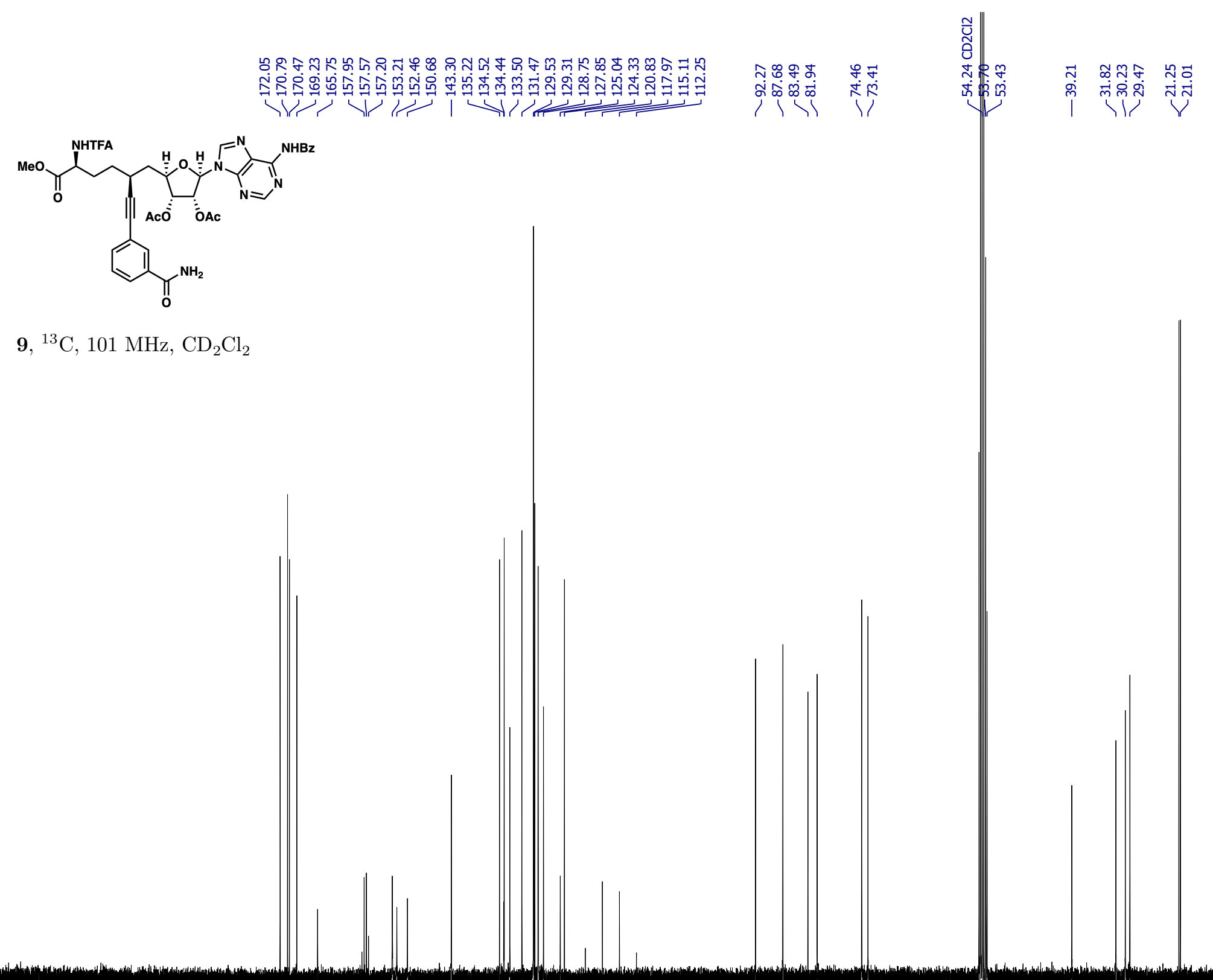




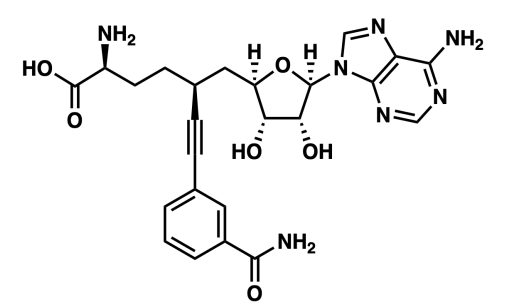

10, ${ }^{1} \mathrm{H}, 600 \mathrm{MHz}, \mathbf{C D}_{\mathbf{3}} \mathbf{C N} / \mathrm{D}_{2} \mathrm{O} / d-\mathrm{TFA}$

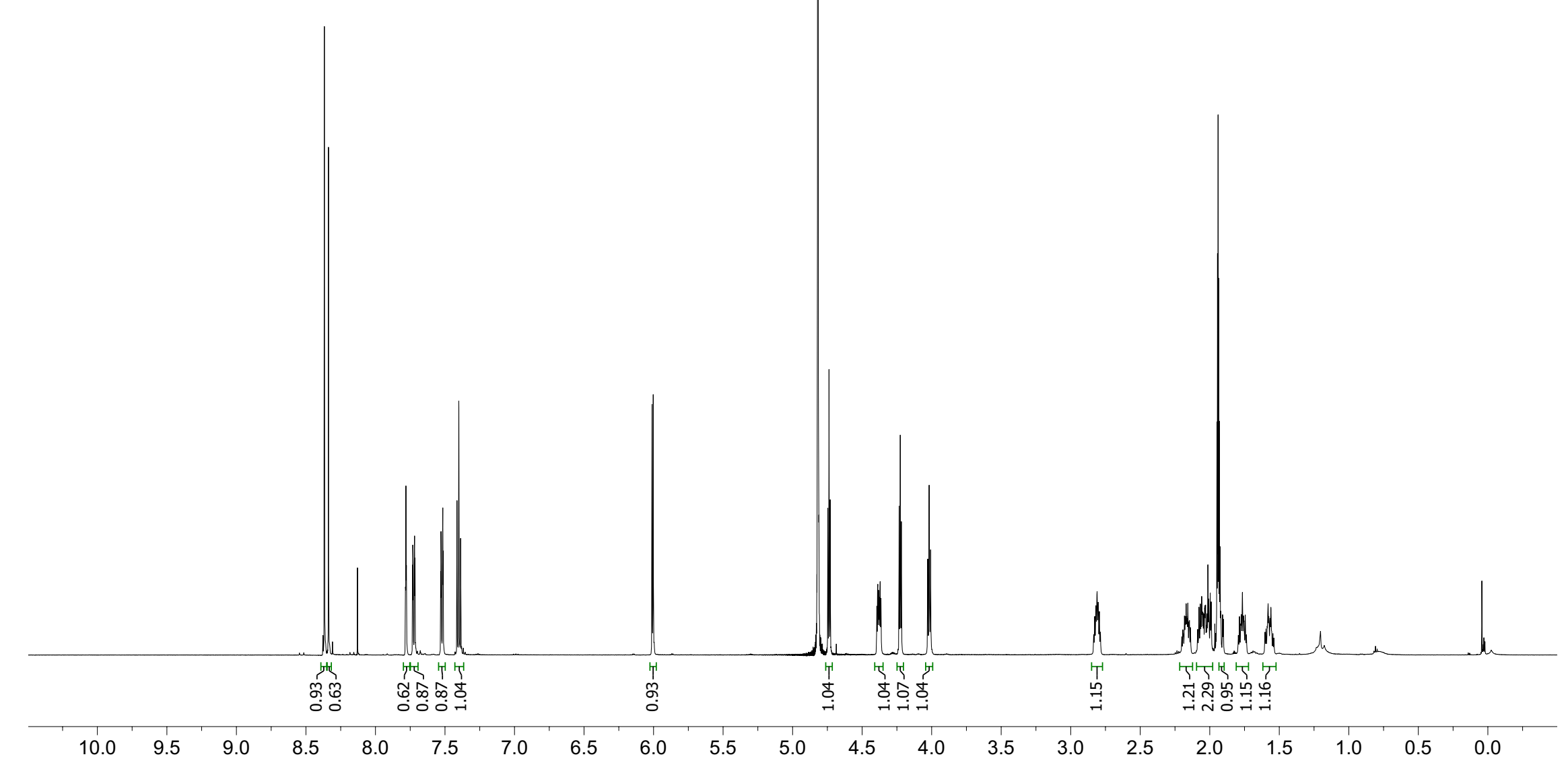




\begin{tabular}{|c|c|c|c|c|c|}
\hline 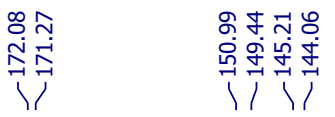 & 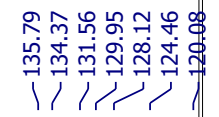 & 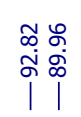 & 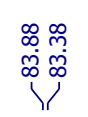 & 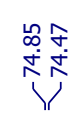 & \\
\hline
\end{tabular}

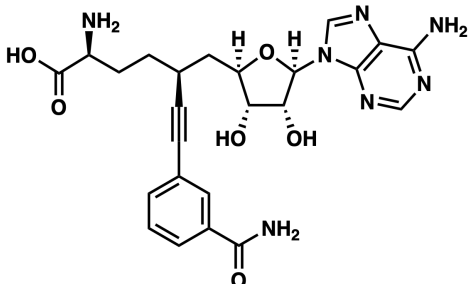

10, ${ }^{13} \mathrm{C}, 101 \mathrm{MHz}, \mathbf{C D}_{3} \mathbf{C N} / \mathrm{D}_{2} \mathrm{O} / d-\mathrm{TFA}$

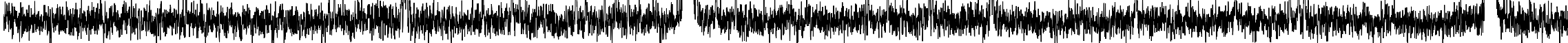

170160

150140

120

$110 \quad 100 \quad 90$

80

70

$60 \quad 50$

$40 \quad 30$

20

$10 \quad 0$ 


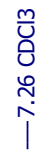

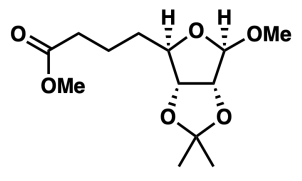

S6, ${ }^{1} \mathrm{H}, 600 \mathrm{MHz}, \mathrm{CDCl}_{3}$

స్తి

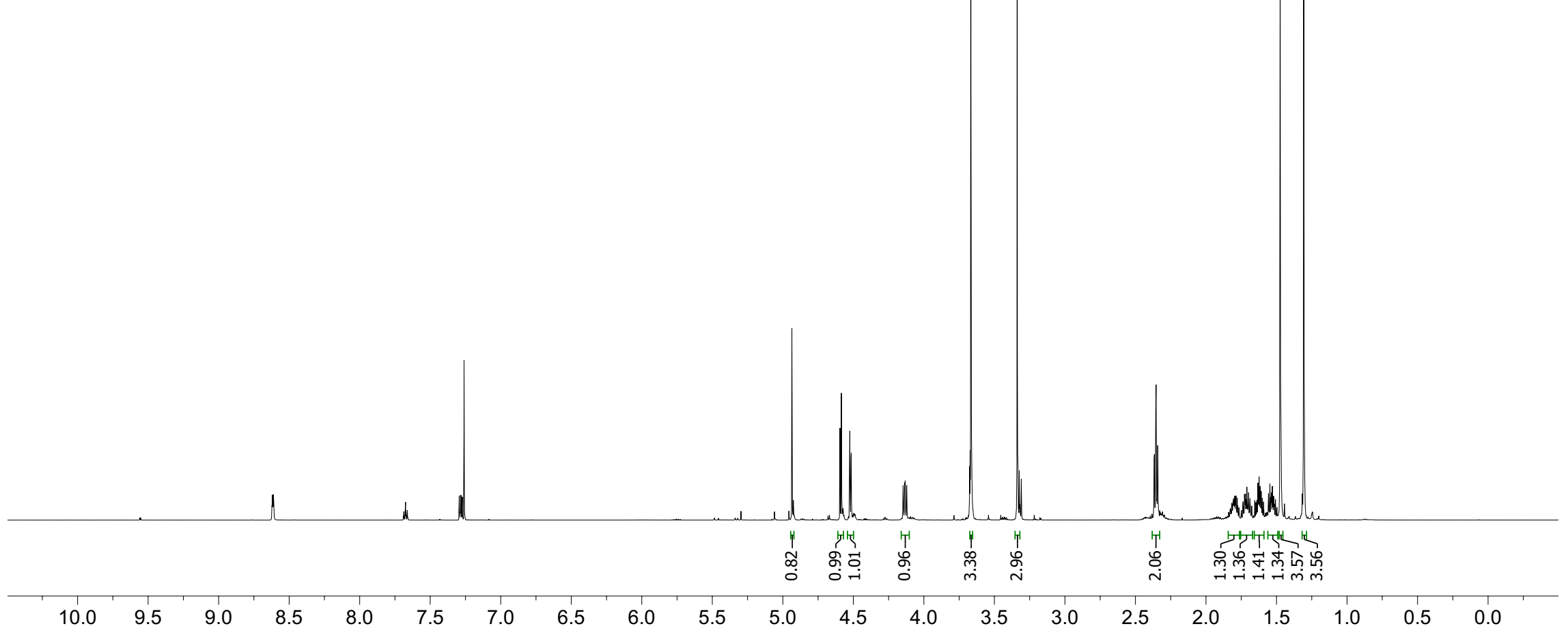




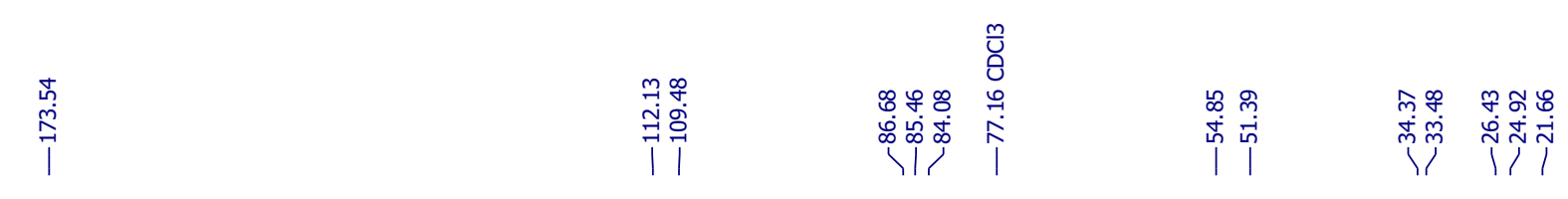

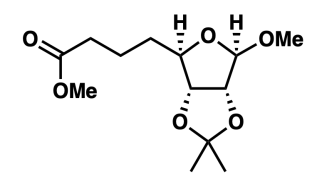

S6, ${ }^{13} \mathrm{C}, 151 \mathrm{MHz}, \mathrm{CDCl}_{3}$

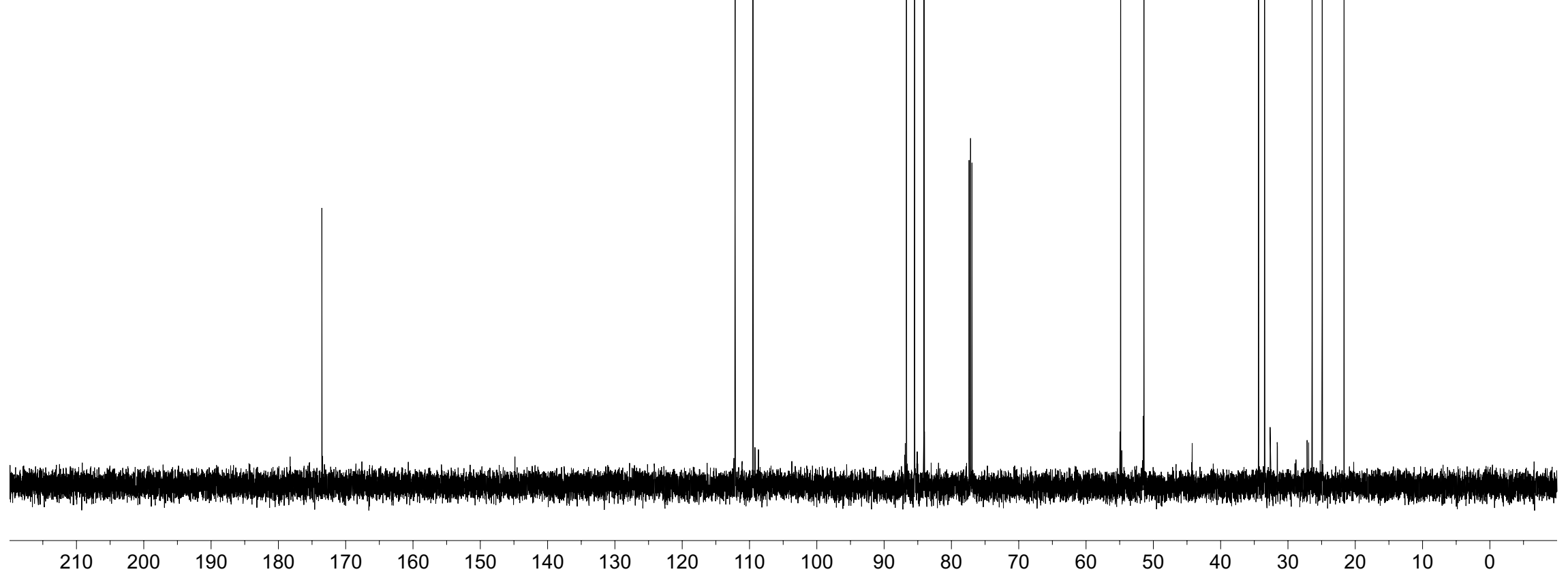




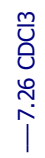

$\stackrel{\mathscr{U}}{\bullet}$

S7, ${ }^{1} \mathrm{H}, 600 \mathrm{MHz}, \mathrm{CDCl}_{3}$

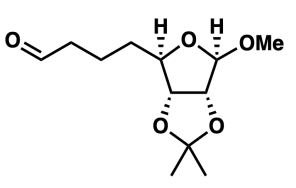

19.0

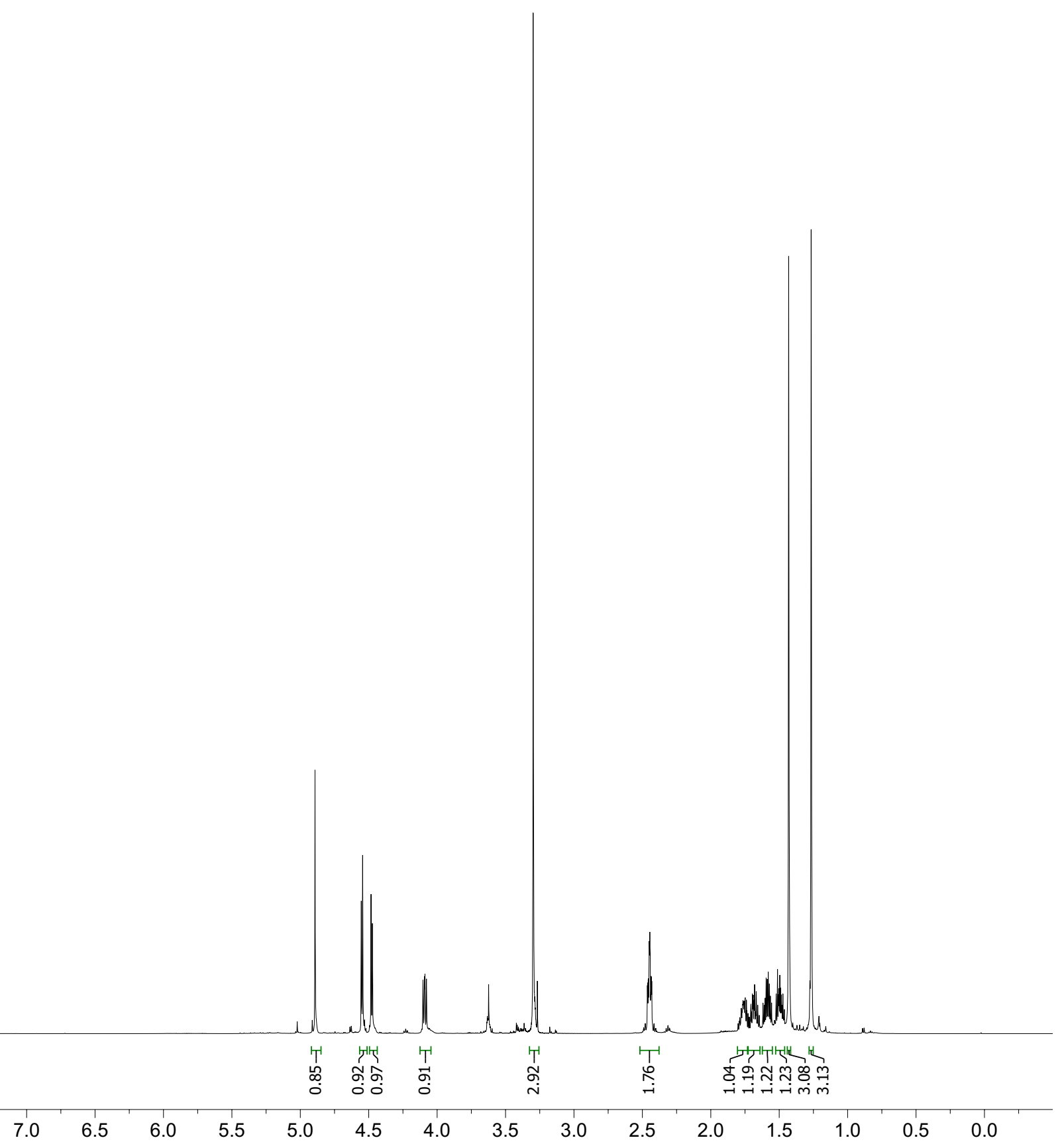




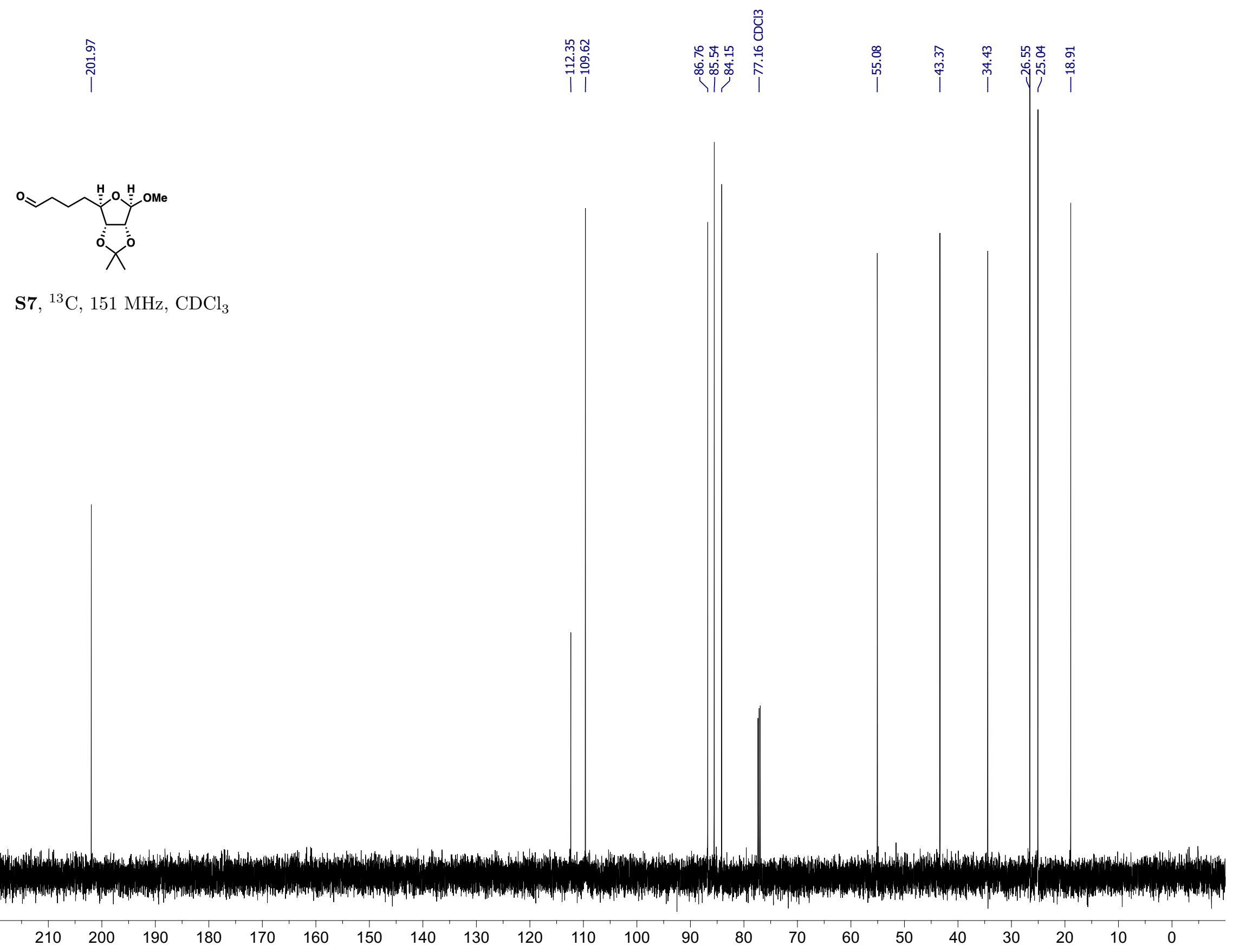




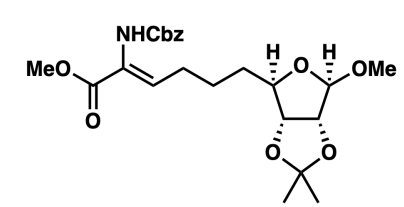

S8, ${ }^{1} \mathrm{H}, 600 \mathrm{MHz}, \mathrm{CDCl}_{3}$

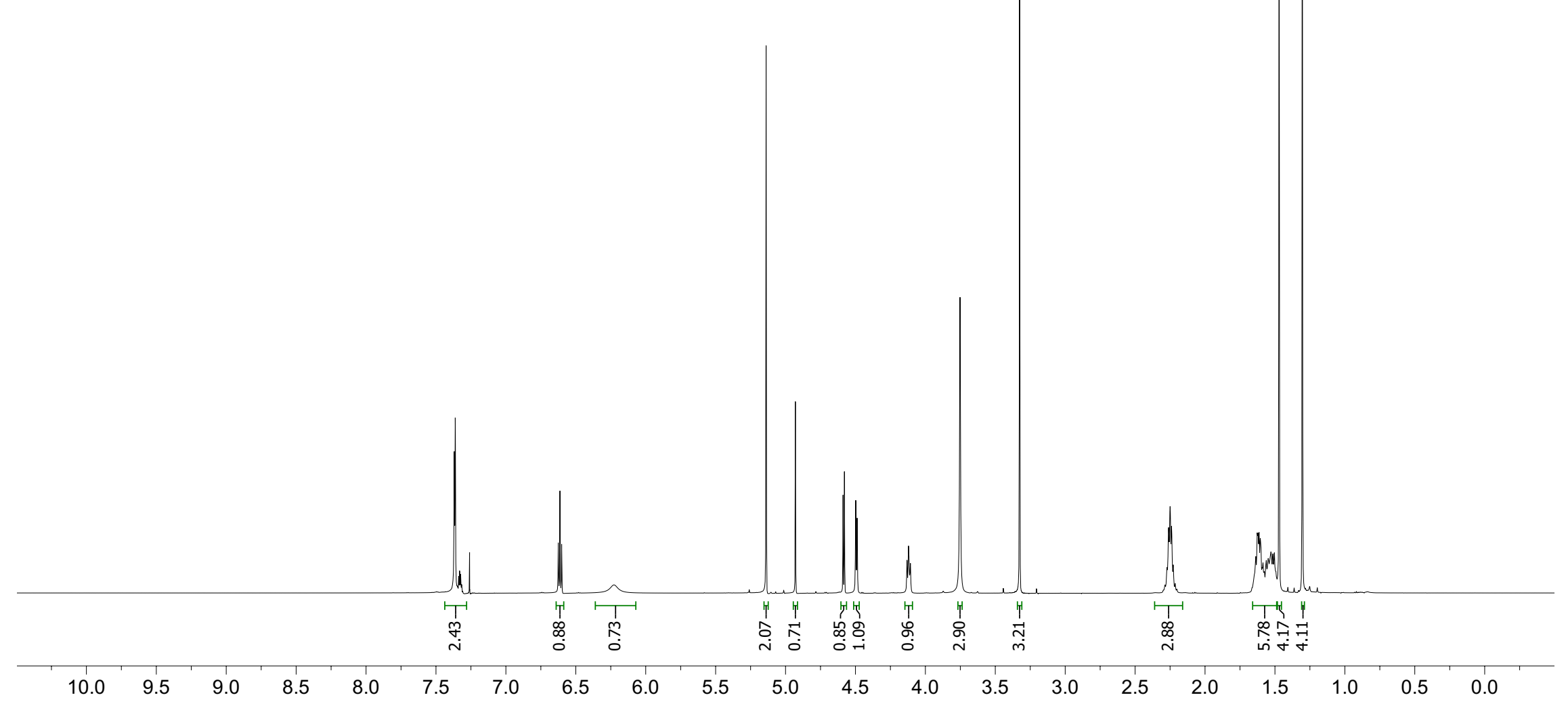




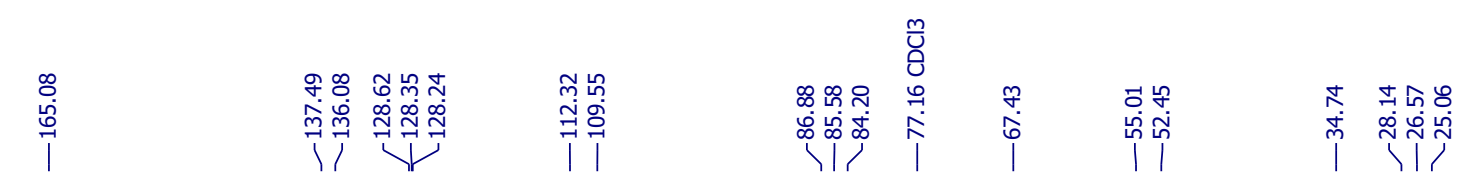

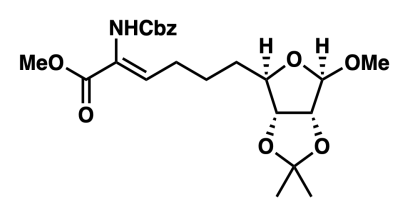

S8, ${ }^{13} \mathrm{C}, 151 \mathrm{MHz}, \mathrm{CDCl}_{3}$ 


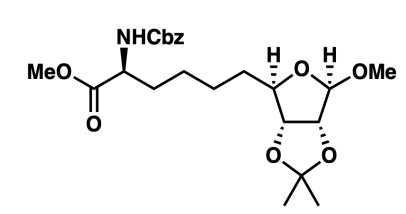

S9, ${ }^{1} \mathrm{H}, 600 \mathrm{MHz}, \mathrm{CDCl}_{3}$

\section{.}

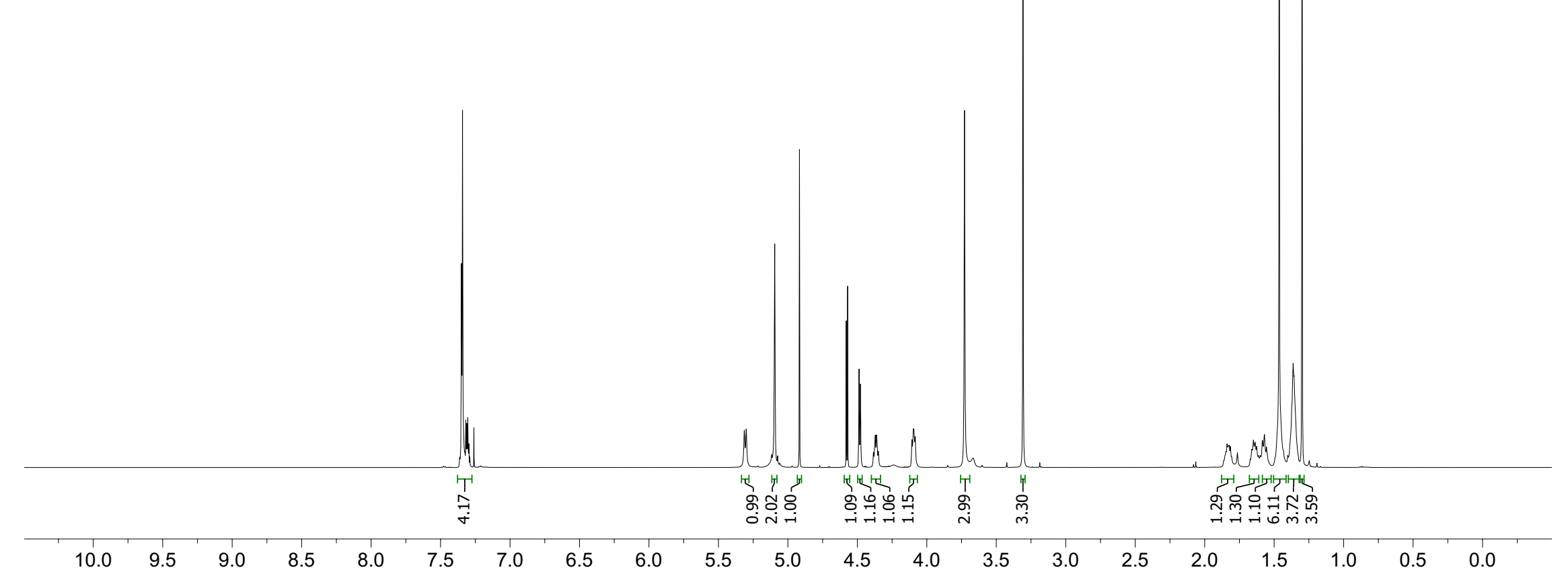




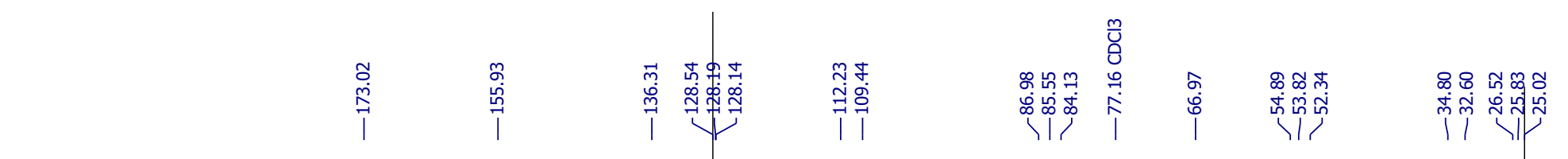

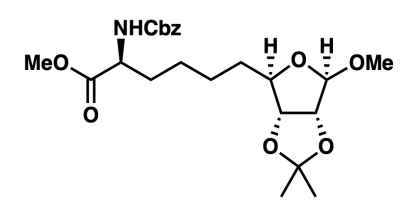

S9, ${ }^{13} \mathrm{C}, 151 \mathrm{MHz}, \mathrm{CDCl}_{3}$ 


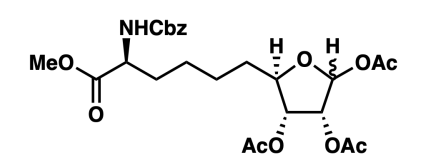

S10, ${ }^{1} \mathrm{H}, 600 \mathrm{MHz}, \mathrm{CDCl}_{3}$

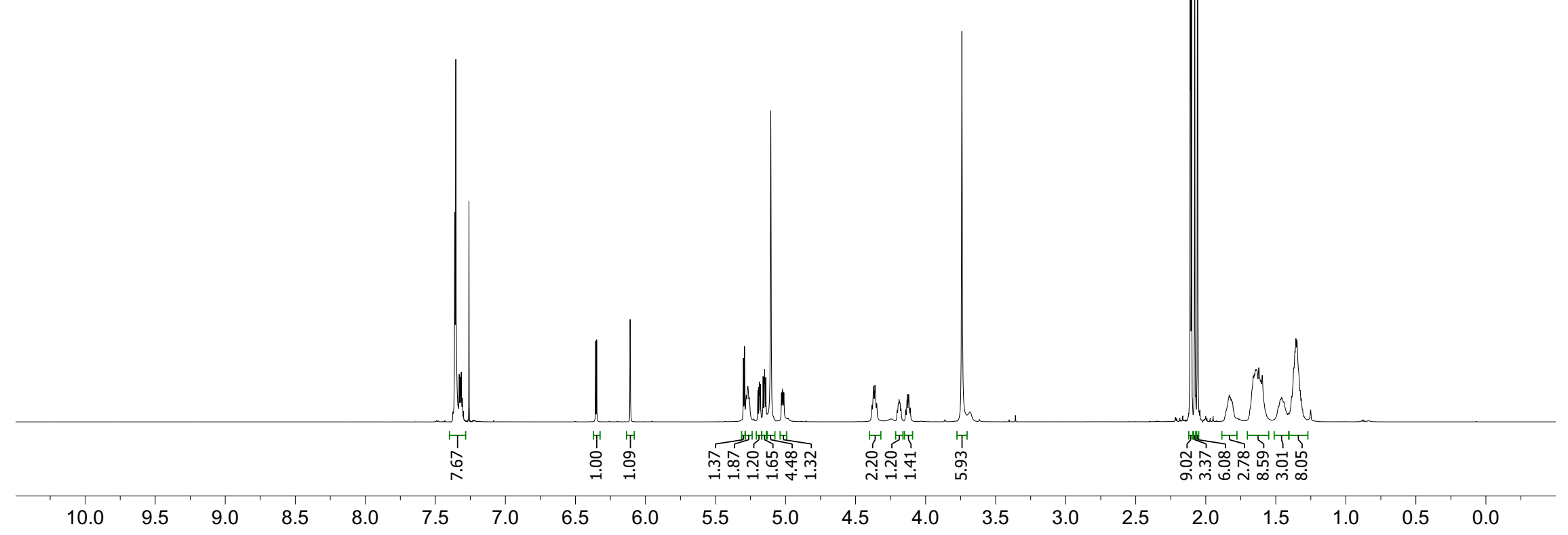




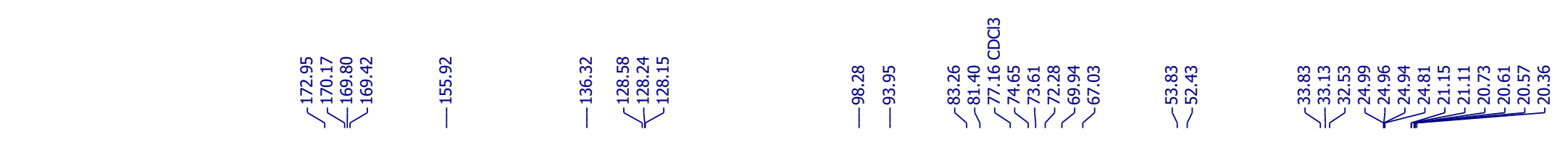

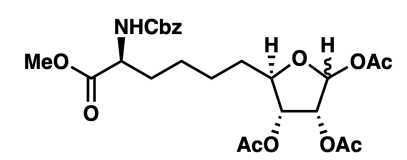

S10, ${ }^{13} \mathrm{C}, 126 \mathrm{MHz}, \mathrm{CDCl}_{3}$ 


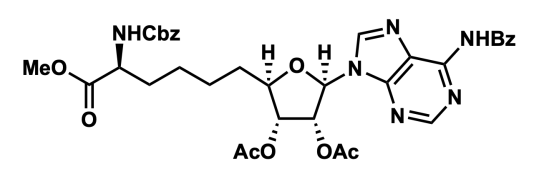

S11, ${ }^{1} \mathrm{H}, 600 \mathrm{MHz}, \mathrm{CDCl}_{3}$

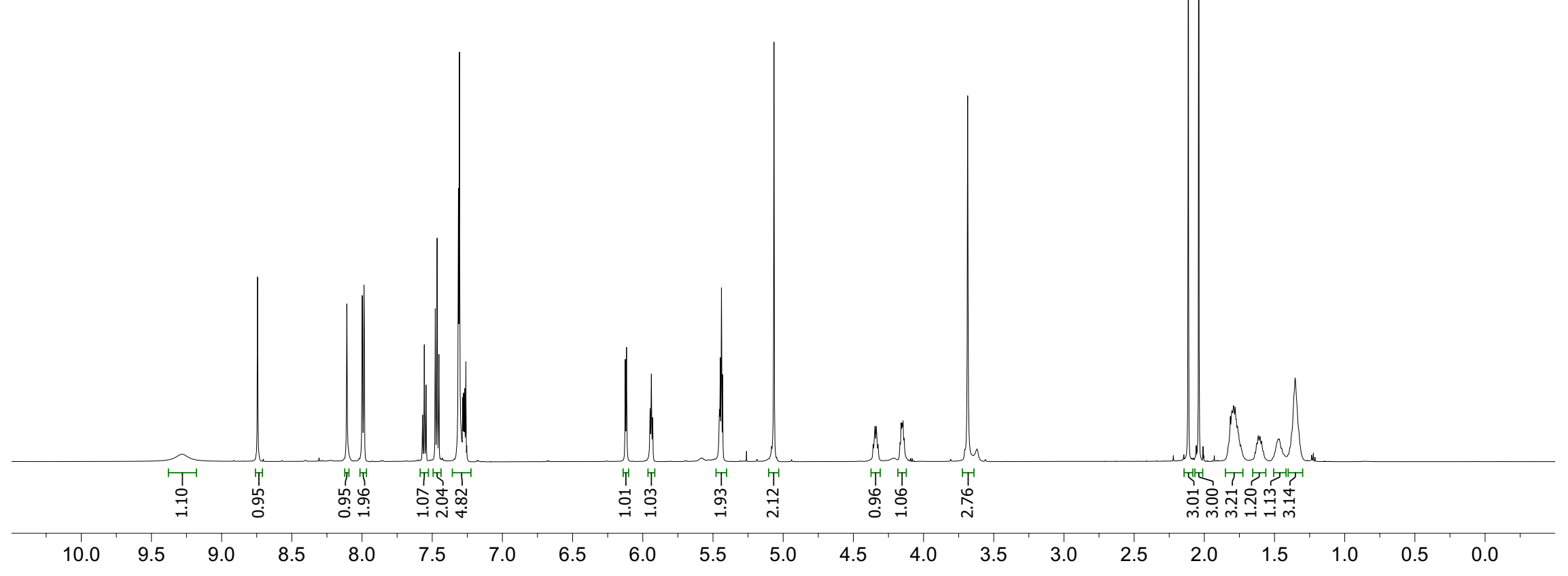



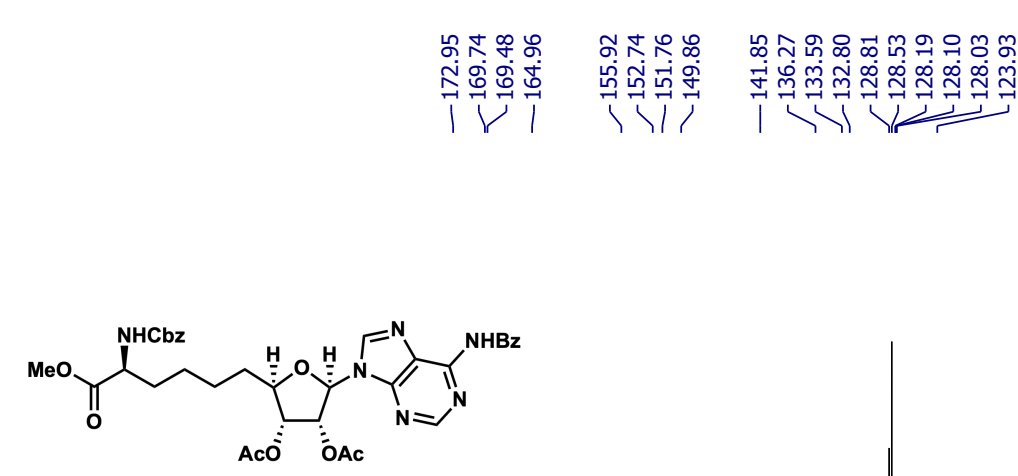

\begin{tabular}{|c|c|c|c|}
\hline 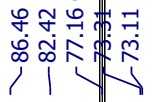 & $\begin{array}{l}\text { ò } \\
\text { o } \\
1\end{array}$ & م. & 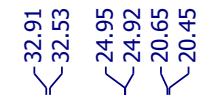 \\
\hline
\end{tabular}

$\mathbf{S 1 1},{ }^{13} \mathrm{C}, 126 \mathrm{MHz}, \mathrm{CDCl}_{3}$

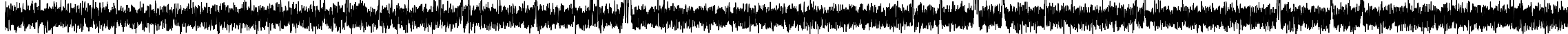




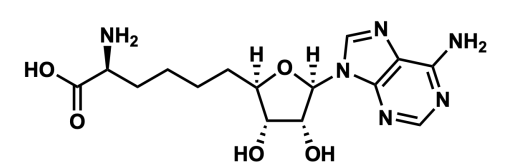

11, ${ }^{1} \mathrm{H}, 600 \mathrm{MHz}, \mathrm{D}_{2} \mathrm{O}$

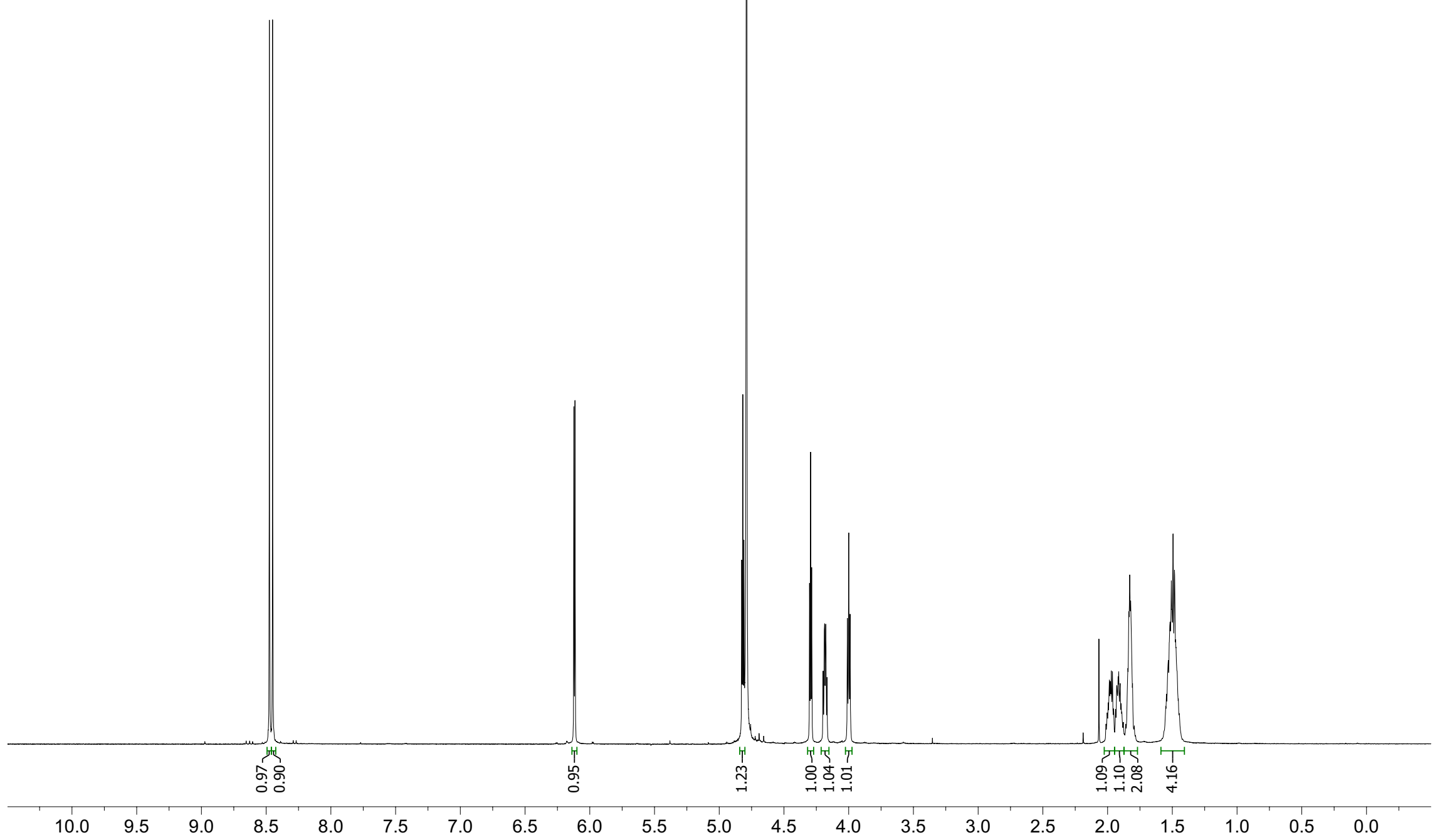




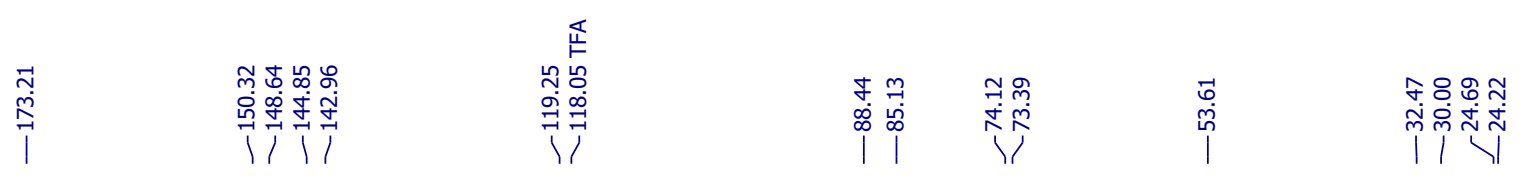

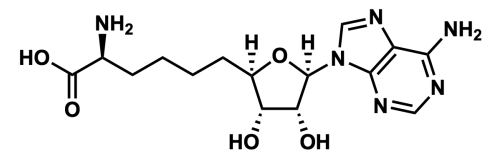

11, ${ }^{13} \mathrm{C}, 101 \mathrm{MHz}, \mathrm{D}_{2} \mathrm{O}$ 


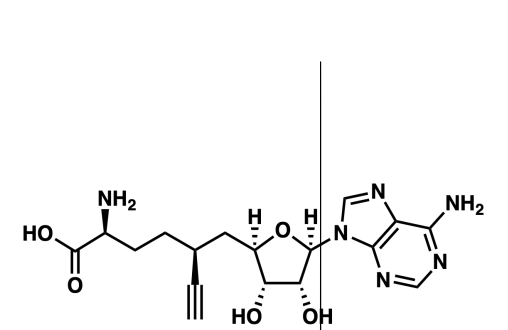

$\mathbf{1 2},{ }^{1} \mathrm{H}, 500 \mathrm{MHz}, \mathrm{CD}_{3} \mathrm{CN} / \mathbf{D}_{\mathbf{2}} \mathbf{O} / d-\mathrm{TFA}$

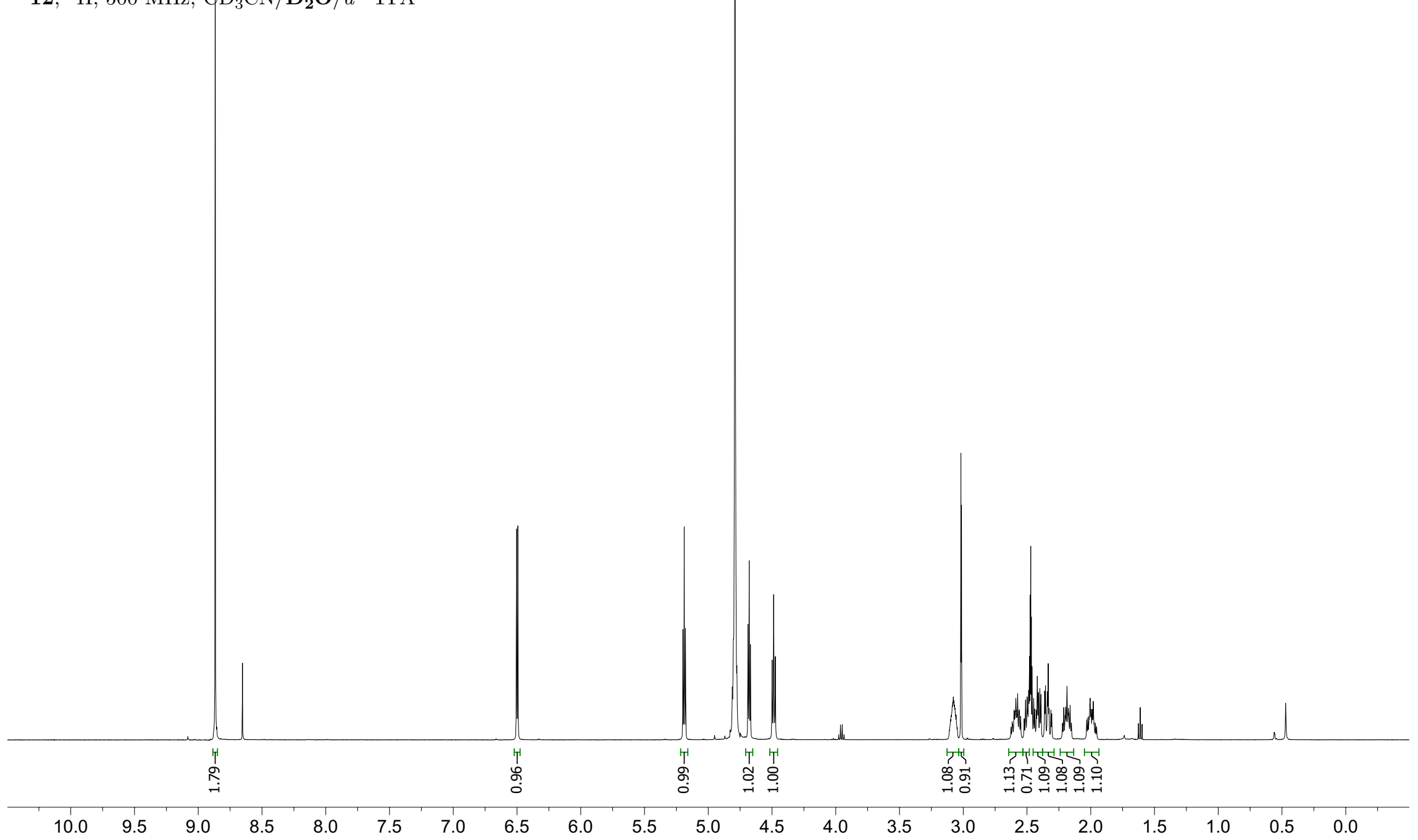




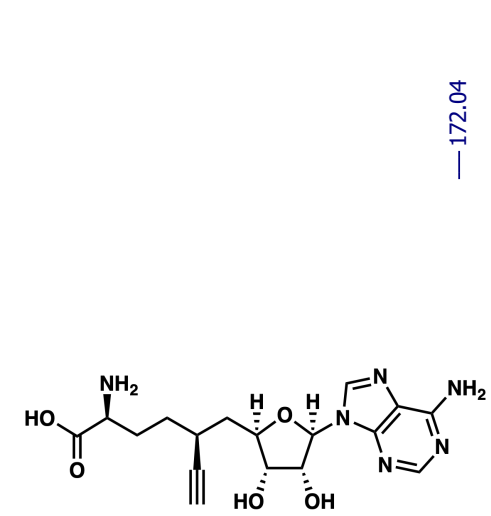

$\mathbf{1 2},{ }^{13} \mathrm{C}, 126 \mathrm{MHz}, \mathbf{C D}_{\mathbf{3}} \mathbf{C N} / \mathrm{D}_{2} \mathrm{O} / d-\mathrm{TFA}$

Mow

$\begin{array}{llll}190 & 180 & 170 & 160\end{array}$

$150 \quad 140$

$130 \quad 120$

$\begin{array}{lll}110 & 100 & 90\end{array}$

$80 \quad 70$

$60 \quad 50$

$40 \quad 30$

$20 \quad 10 \quad 0$ 


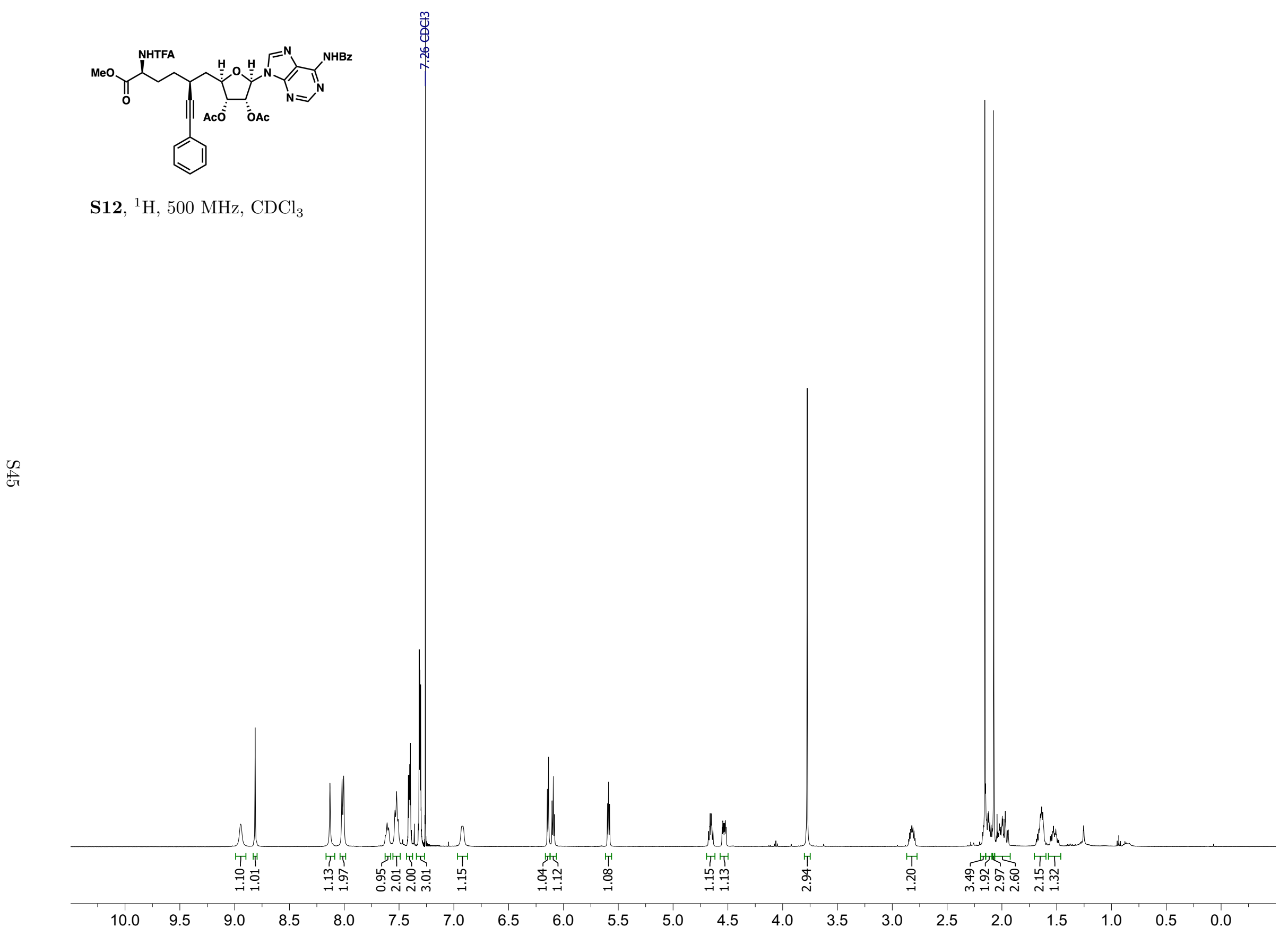




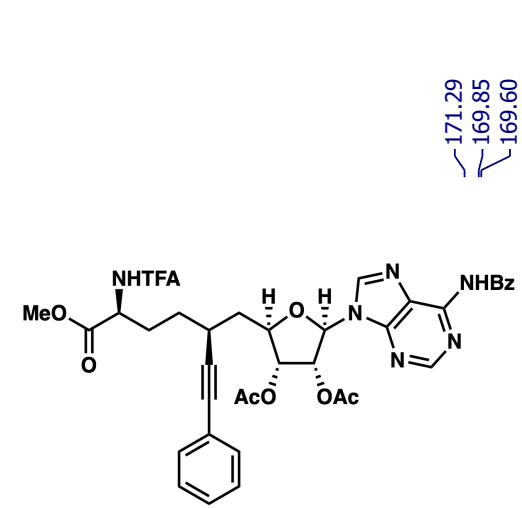

要

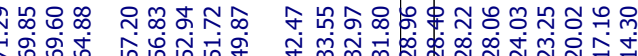

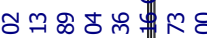
वंmmin

$\frac{20}{4}$

î

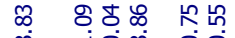

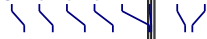

ला1

S12 ${ }^{13} \mathrm{C}, 101 \mathrm{MHz}, \mathrm{CDCl}_{3}$

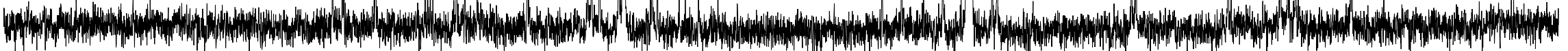

170160

$150 \quad 140$

130

20

90

$80 \quad 70$

60

50

$40 \quad 30$

20 


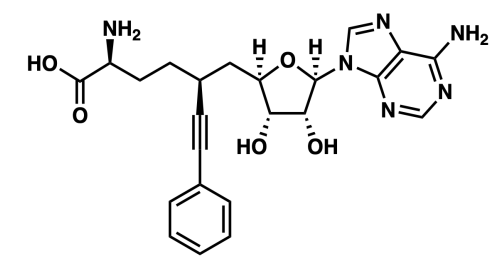

13, ${ }^{1} \mathrm{H}, 600 \mathrm{MHz}, \mathrm{CD}_{3} \mathrm{CN} / \mathbf{D}_{\mathbf{2}} \mathbf{O} / d-\mathrm{TFA}$

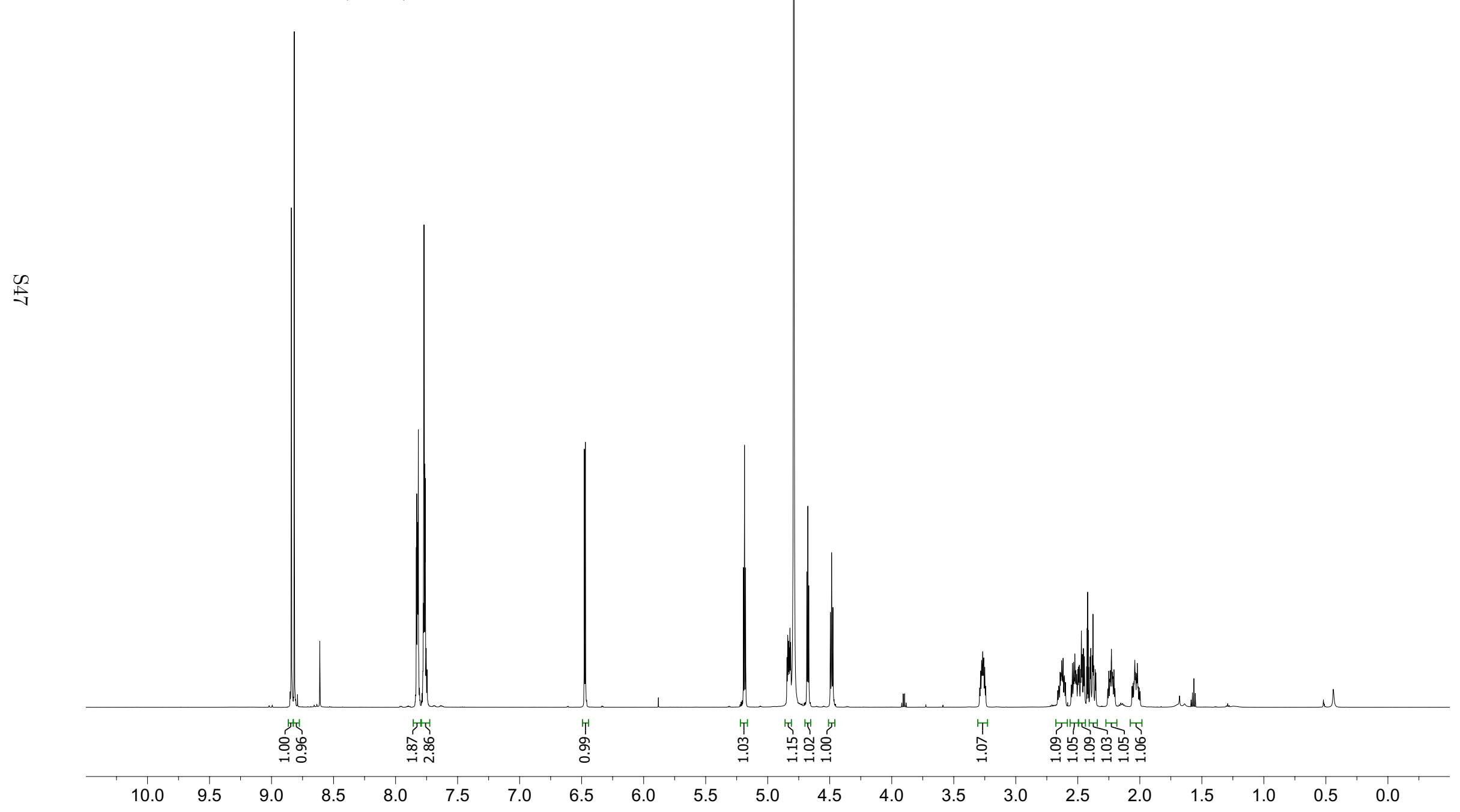




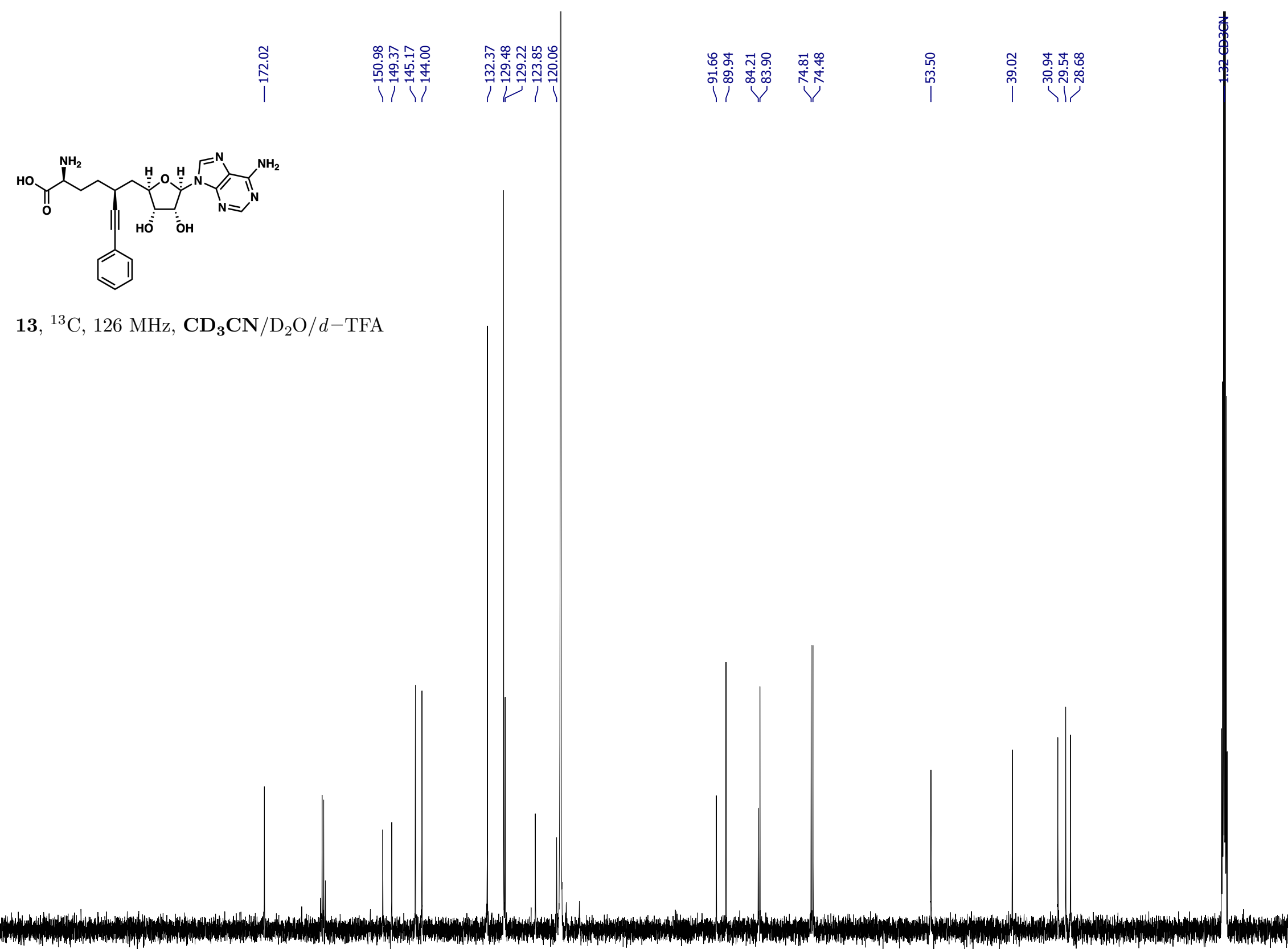

$210 \quad 200$

$170 \quad 160$

$150 \quad 140$

$130 \quad 120$

$\begin{array}{lll}110 & 100 \quad 90\end{array}$

$80 \quad 70$

$60 \quad 50$

$40 \quad 30$

$20 \quad 10$ 


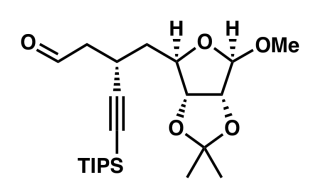

S13, ${ }^{1} \mathrm{H}, 600 \mathrm{MHz}, \mathrm{CDCl}_{3}$

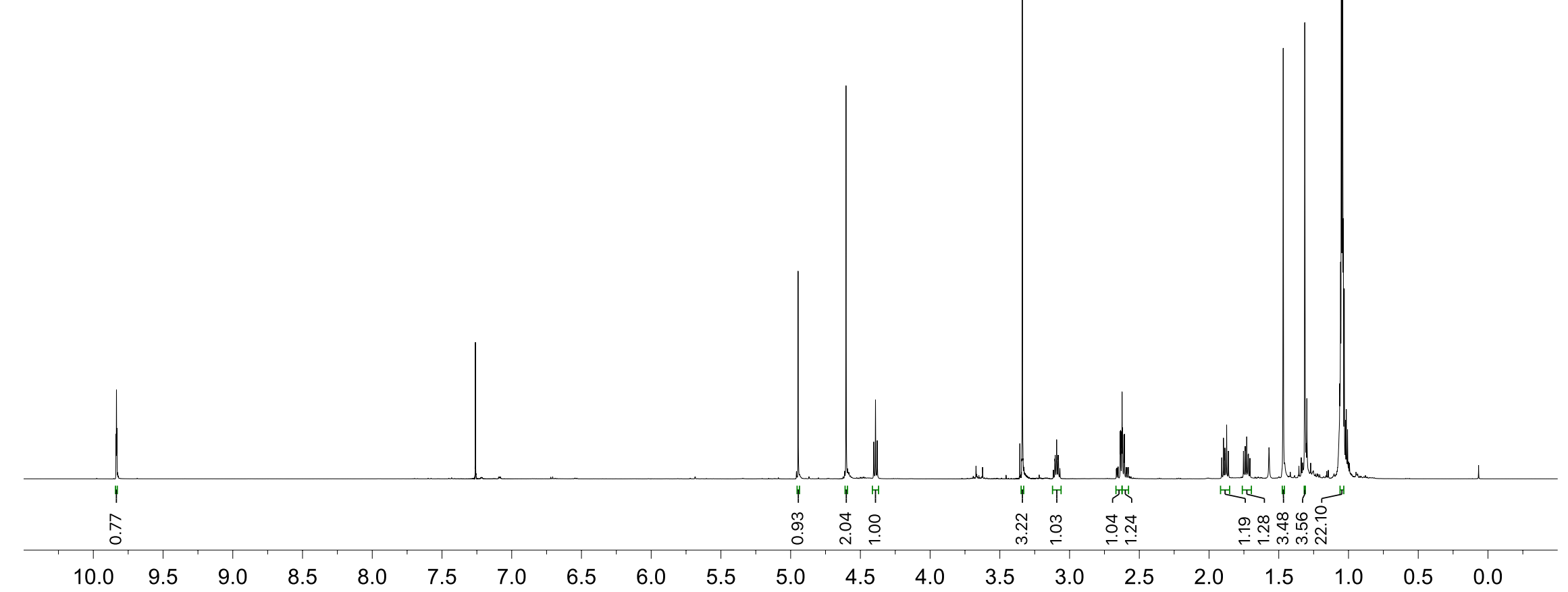




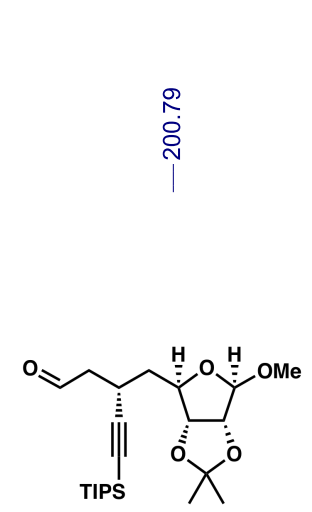

S13, ${ }^{13} \mathrm{C}, 126 \mathrm{MHz}, \mathrm{CDCl}_{3}$

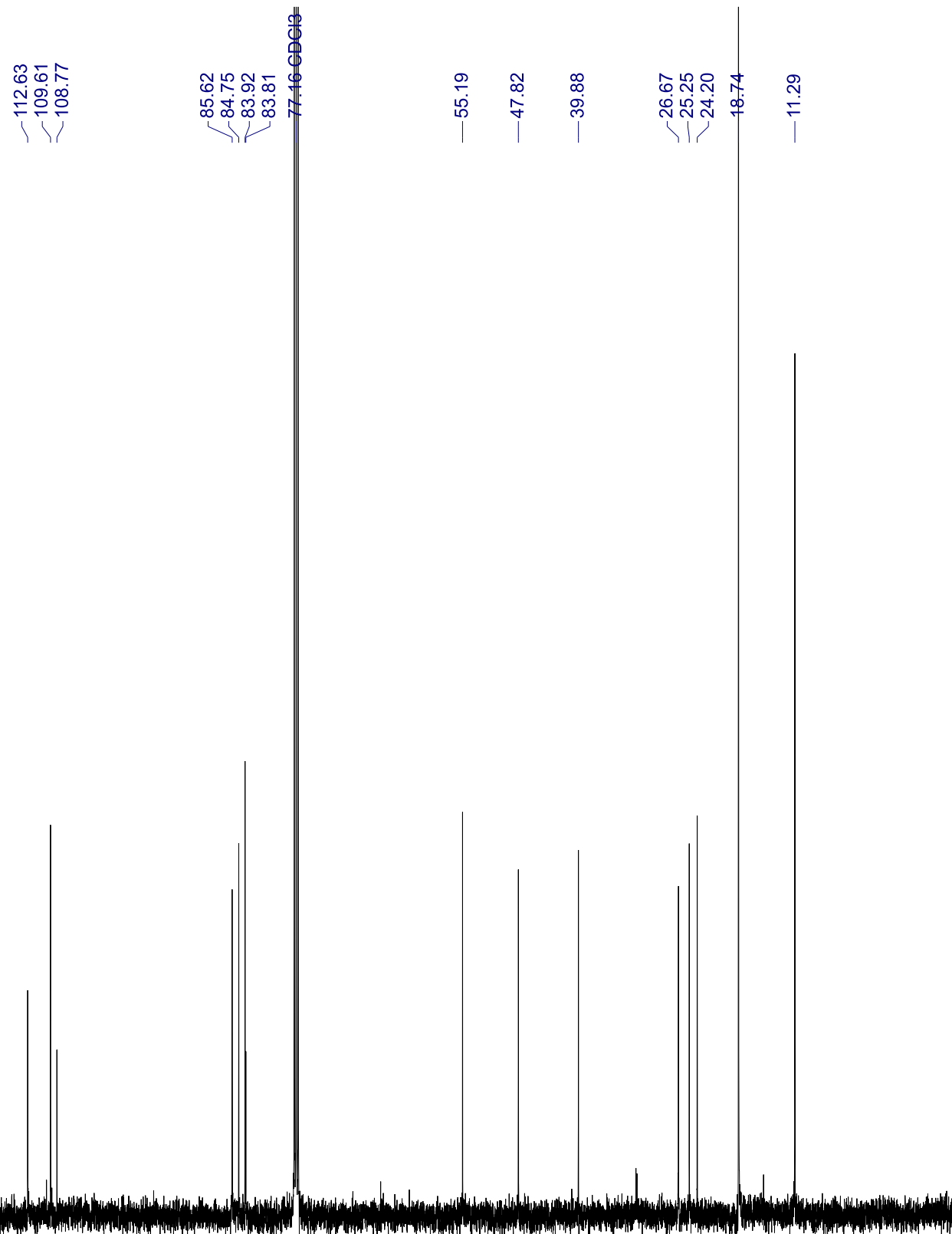




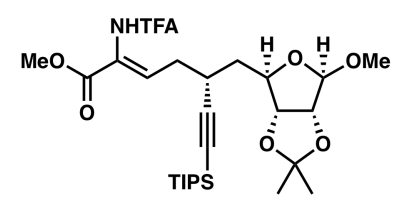

$\frac{m}{0}$
0
0
$\stackrel{0}{i}$
$i$

$\mathbf{S 1 4},{ }^{1} \mathrm{H}, 600 \mathrm{MHz}, \mathrm{CDCl}_{3}$

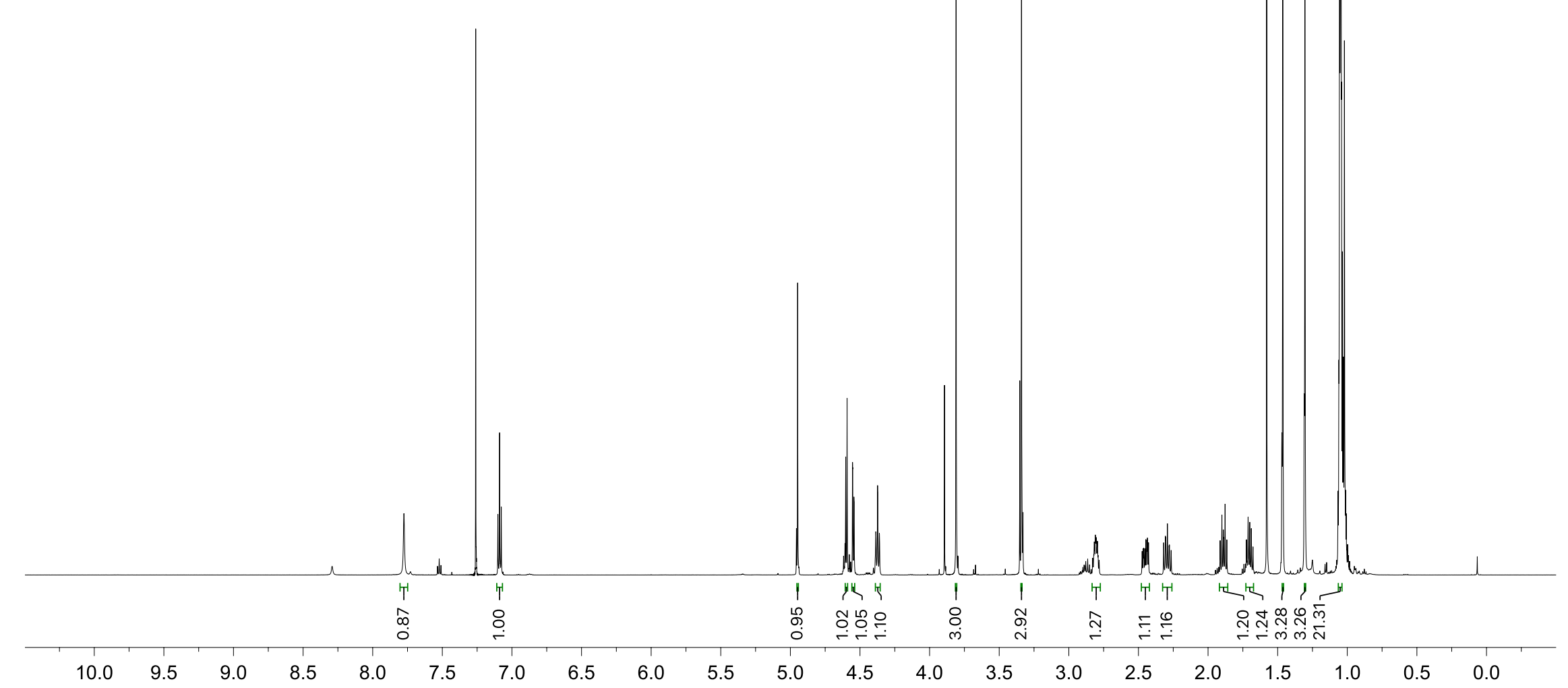



กิ ㅅำ

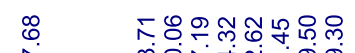

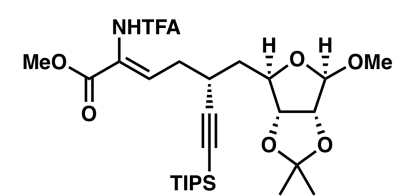

S14, ${ }^{13} \mathrm{C}, 101 \mathrm{MHz}, \mathrm{CDCl}_{3}$

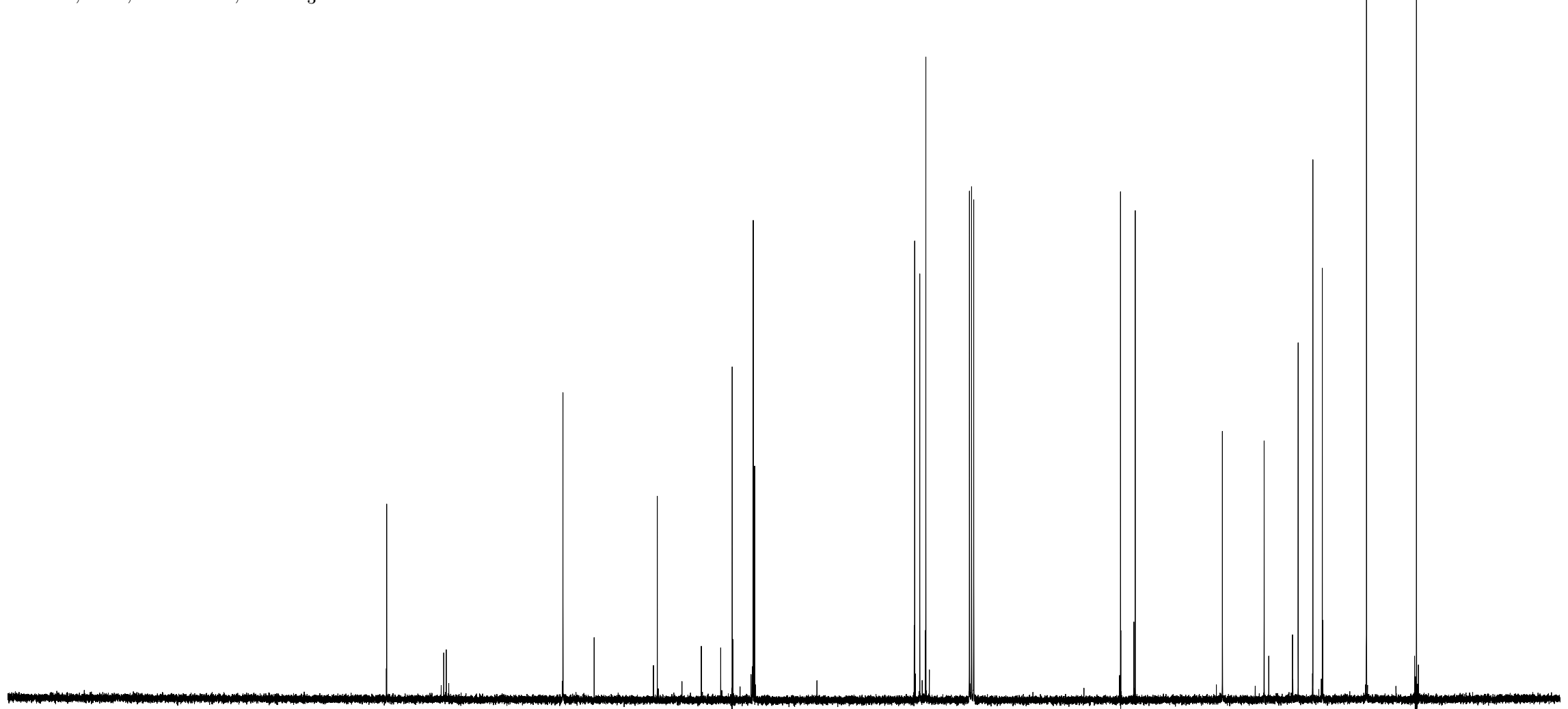




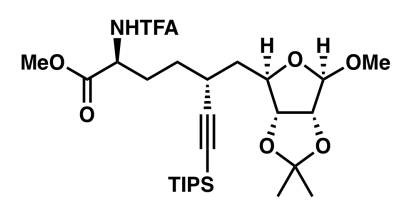

$\mathbf{S 1 5},{ }^{1} \mathrm{H}, 500 \mathrm{MHz}, \mathrm{CDCl}_{3}$

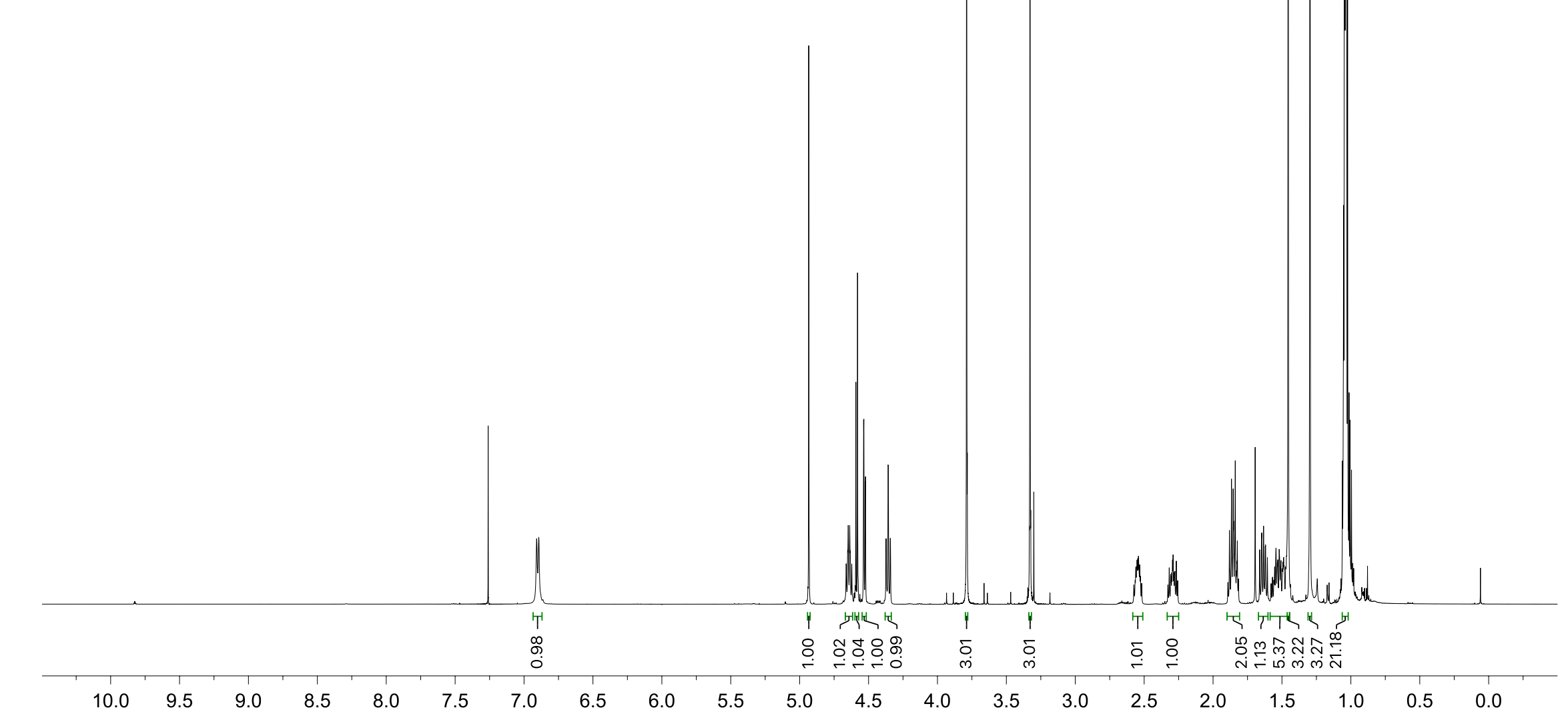




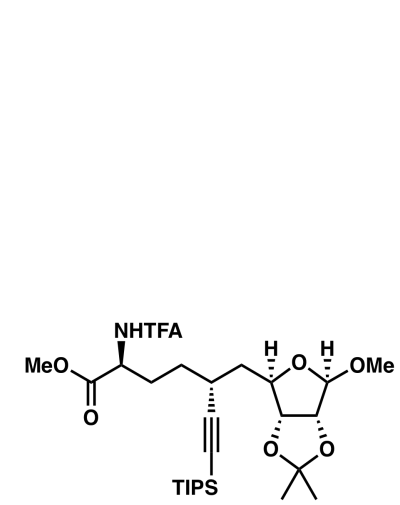

S15, ${ }^{13} \mathrm{C}, 126 \mathrm{MHz}, \mathrm{CDCl}_{3}$

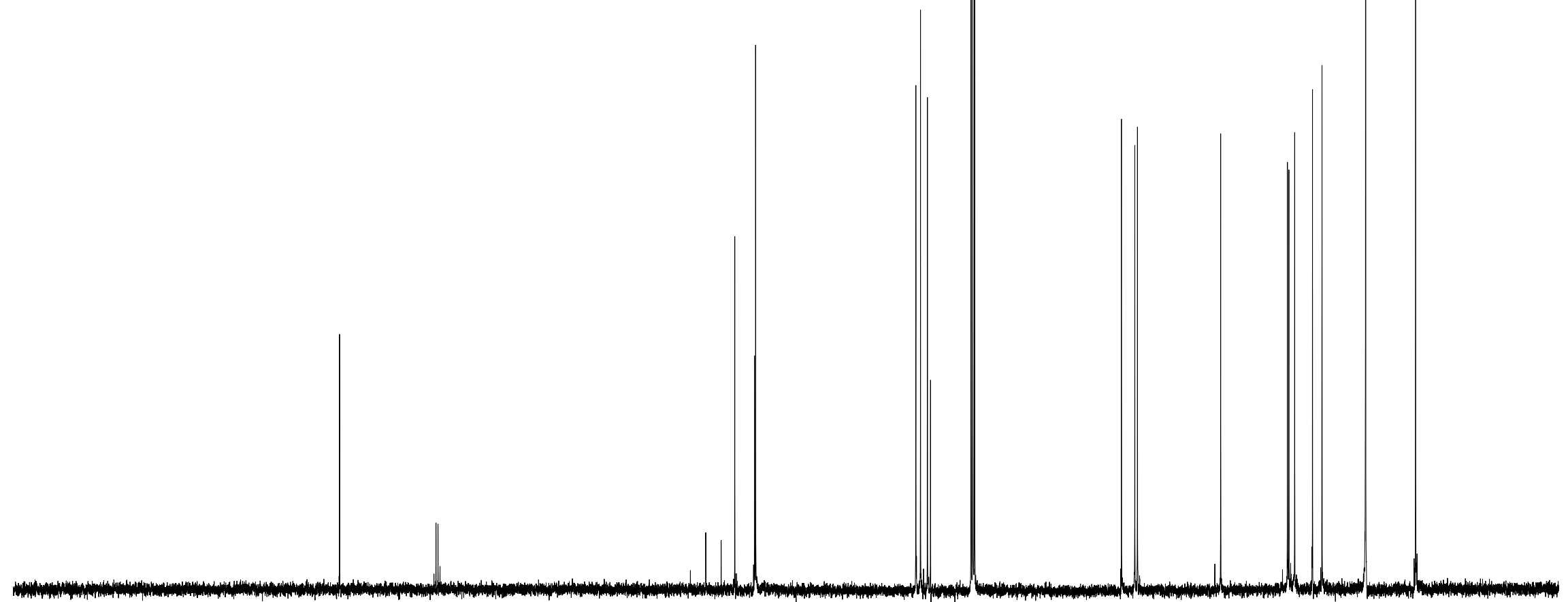




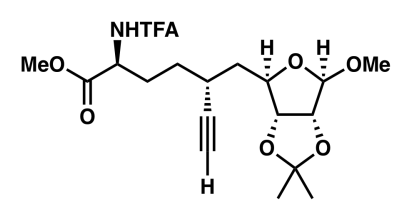

S16, ${ }^{1} \mathrm{H}, 500 \mathrm{MHz}, \mathrm{CDCl}_{3}$

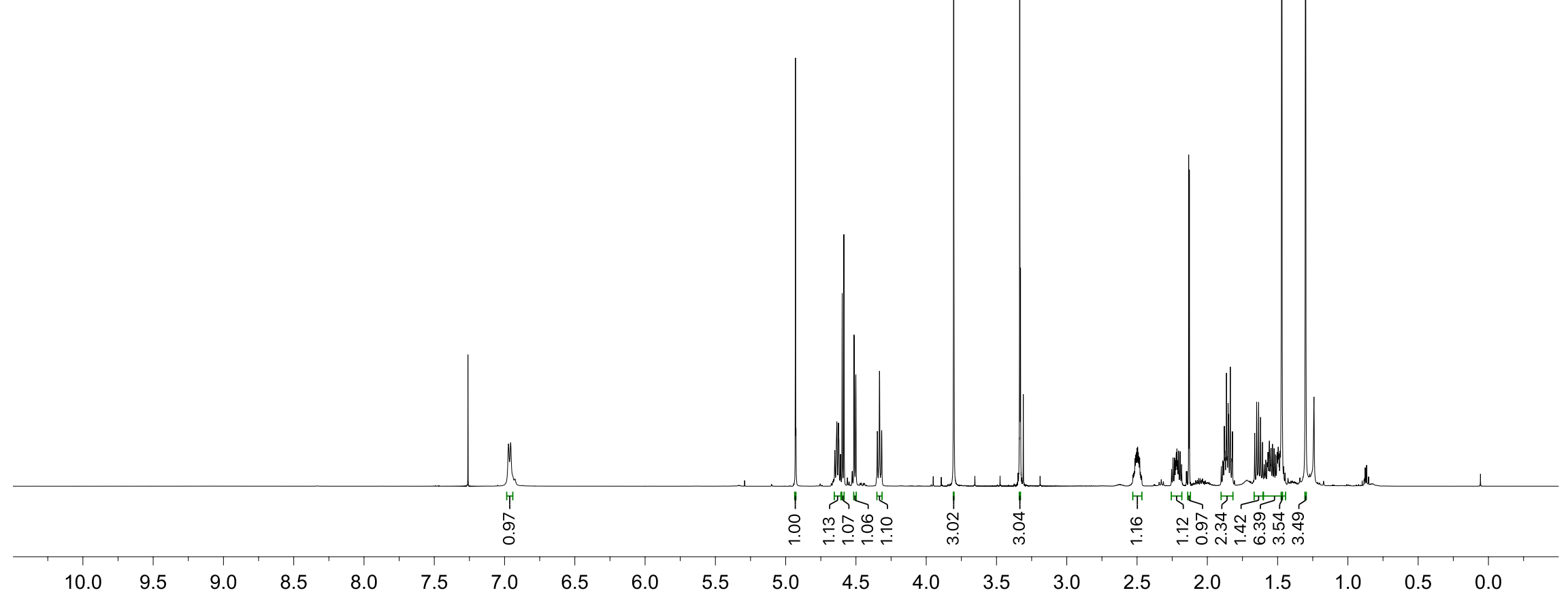




il

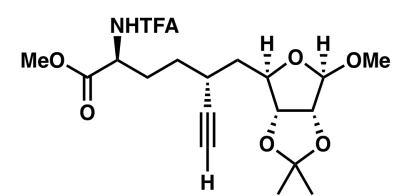

S16, ${ }^{13} \mathrm{C}, 126 \mathrm{MHz}, \mathrm{CDCl}_{3}$ 


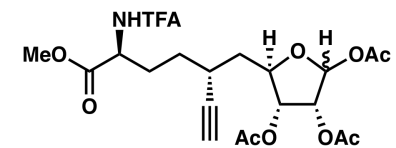

$\mathbf{S 1 7},{ }^{1} \mathrm{H}, 500 \mathrm{MHz}, \mathrm{CDCl}_{3}$

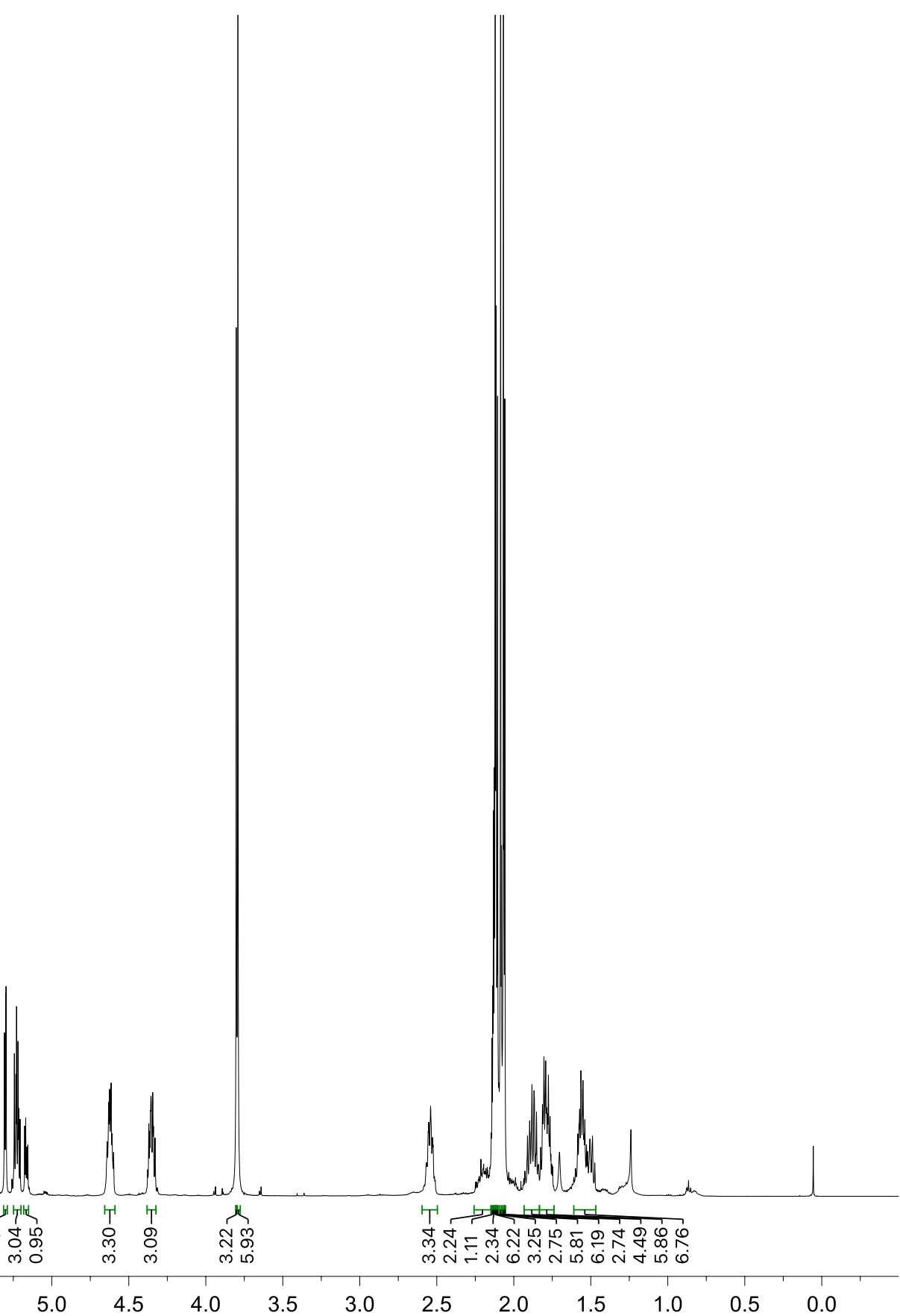




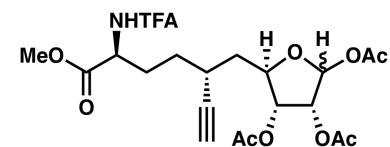

$\mathbf{S 1 7},{ }^{13} \mathrm{C}, 126 \mathrm{MHz}, \mathrm{CDCl}_{3}$

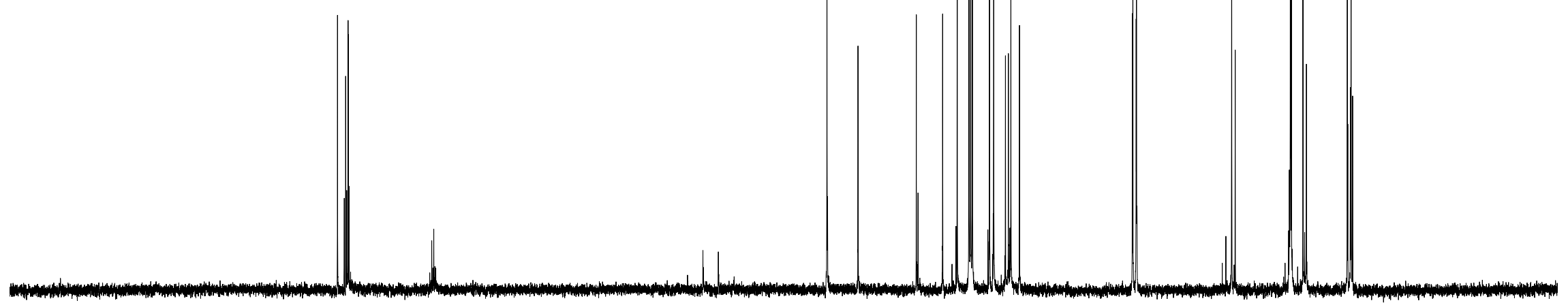




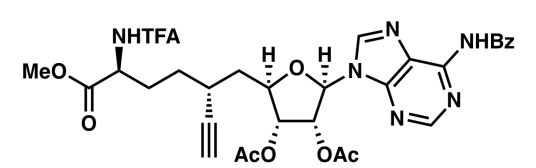

S18, ${ }^{1} \mathrm{H}, 500 \mathrm{MHz}, \mathrm{CDCl}_{3}$

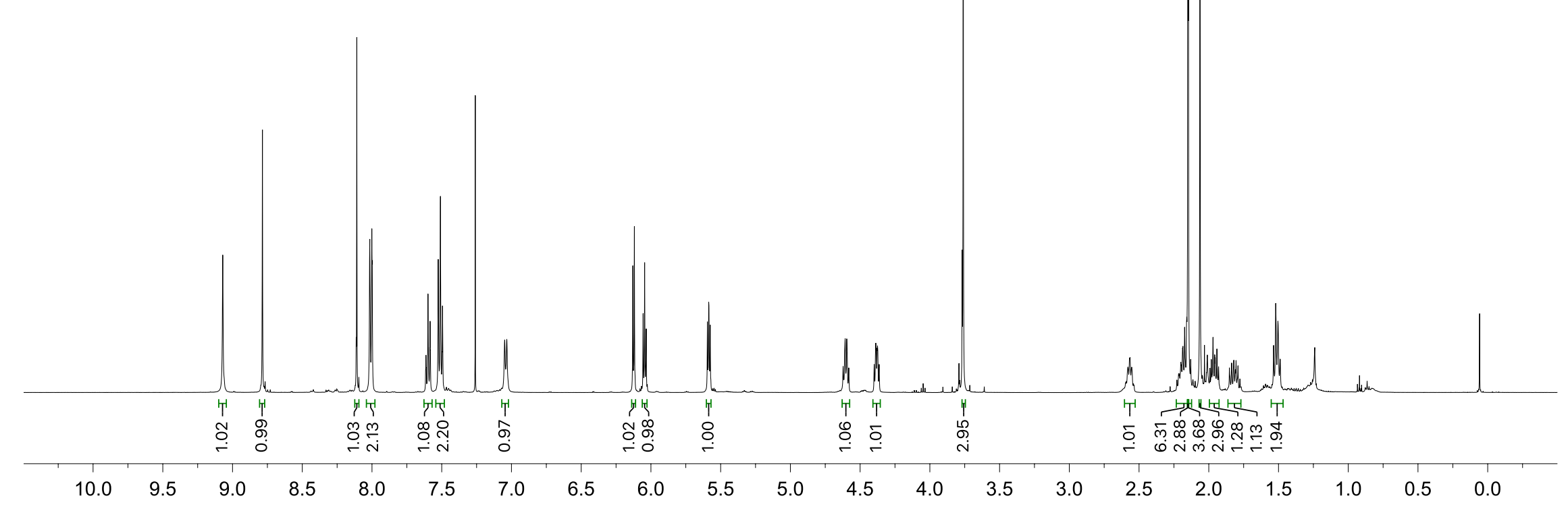




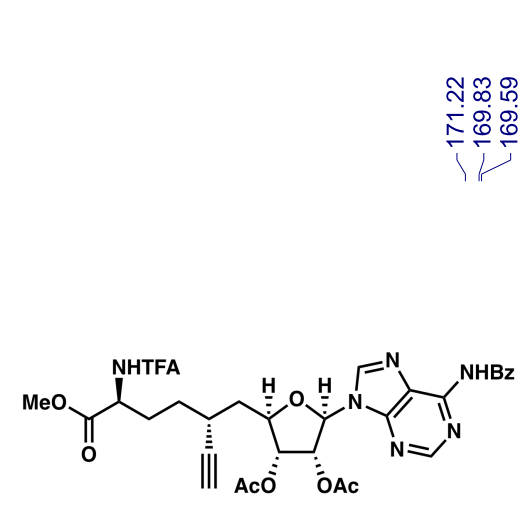

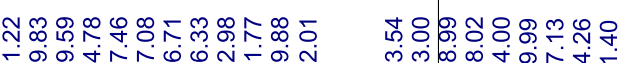

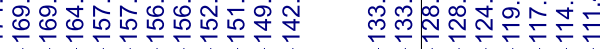

응

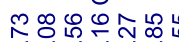
काष्र

$ㅇ ㅡ$

$11>1<1$

น)

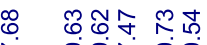

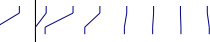

S18, ${ }^{13} \mathrm{C}, 100 \mathrm{MHz}, \mathrm{CDCl}_{3}$

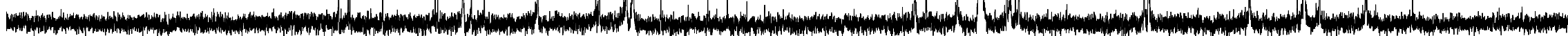




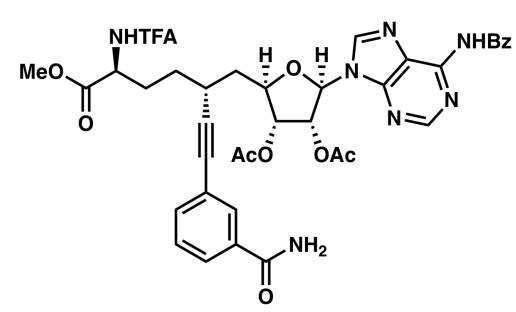

S19, ${ }^{1} \mathrm{H}, 600 \mathrm{MHz}, \mathrm{CDCl}_{3}$

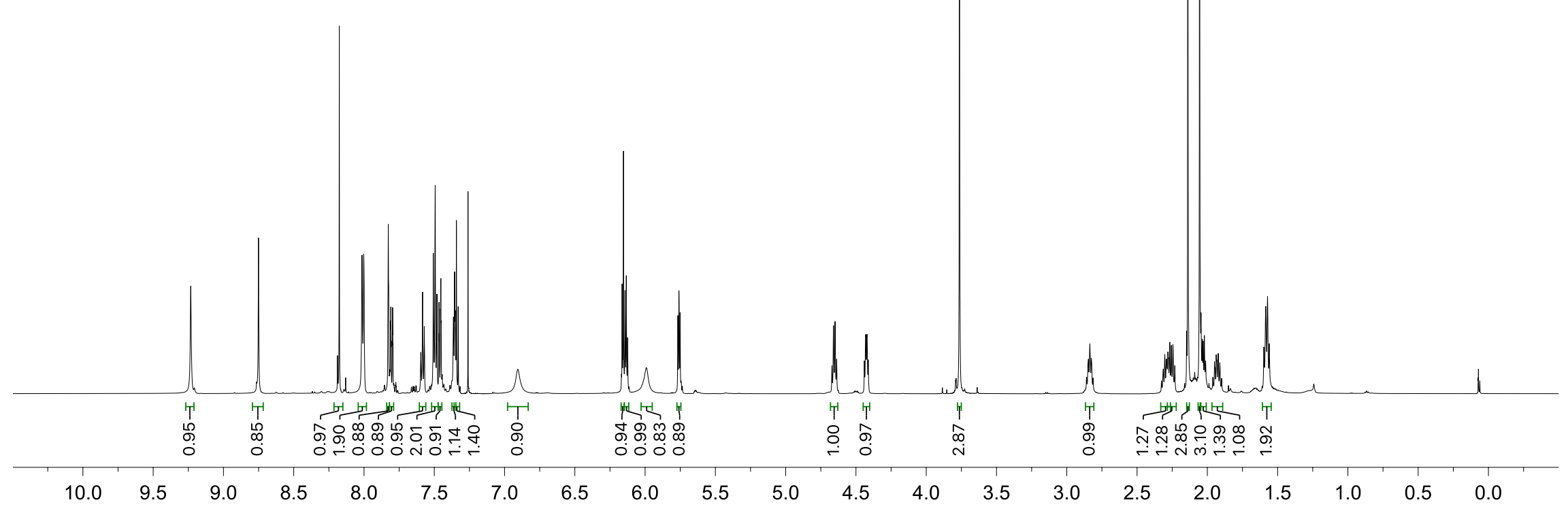




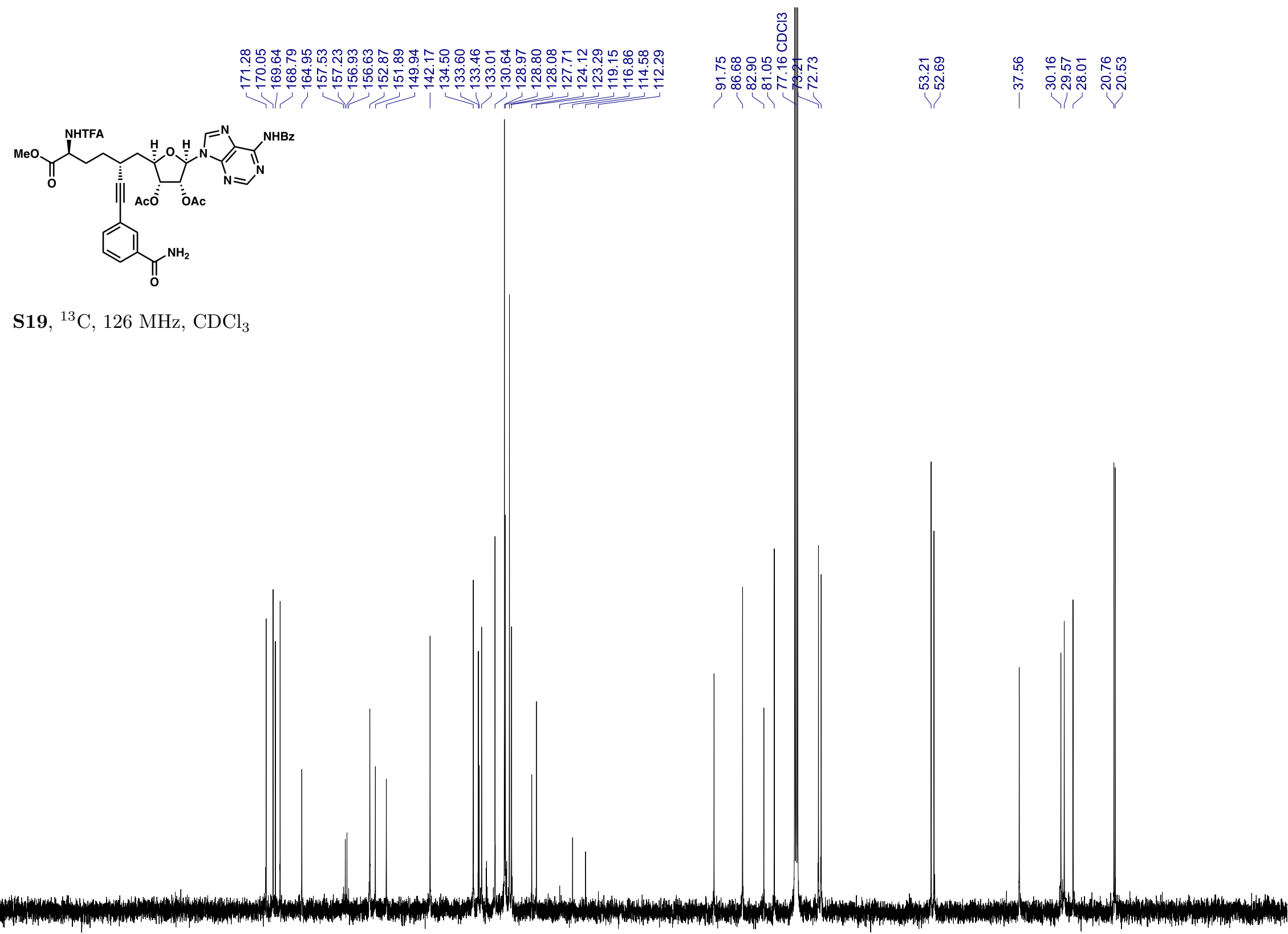

$210 \quad 200$ 


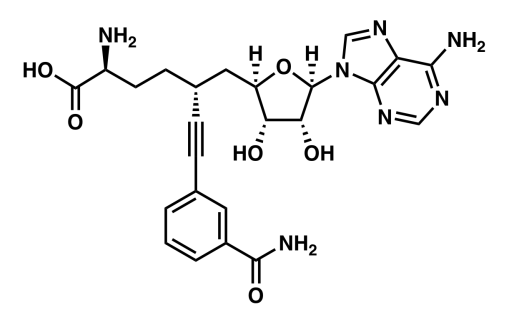

14, ${ }^{1} \mathrm{H}, 600 \mathrm{MHz}, \mathbf{C D}_{\mathbf{3}} \mathbf{C N} / \mathrm{D}_{2} \mathrm{O} / d-\mathrm{TFA}$

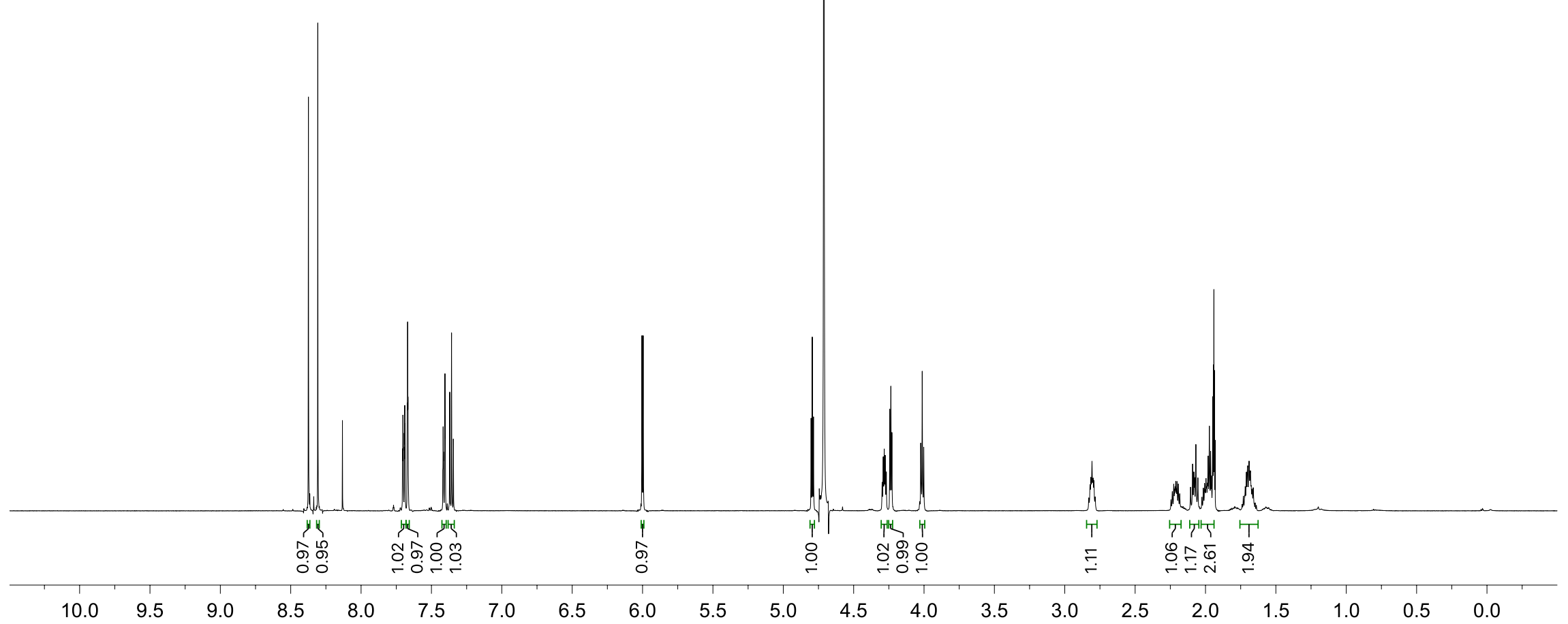




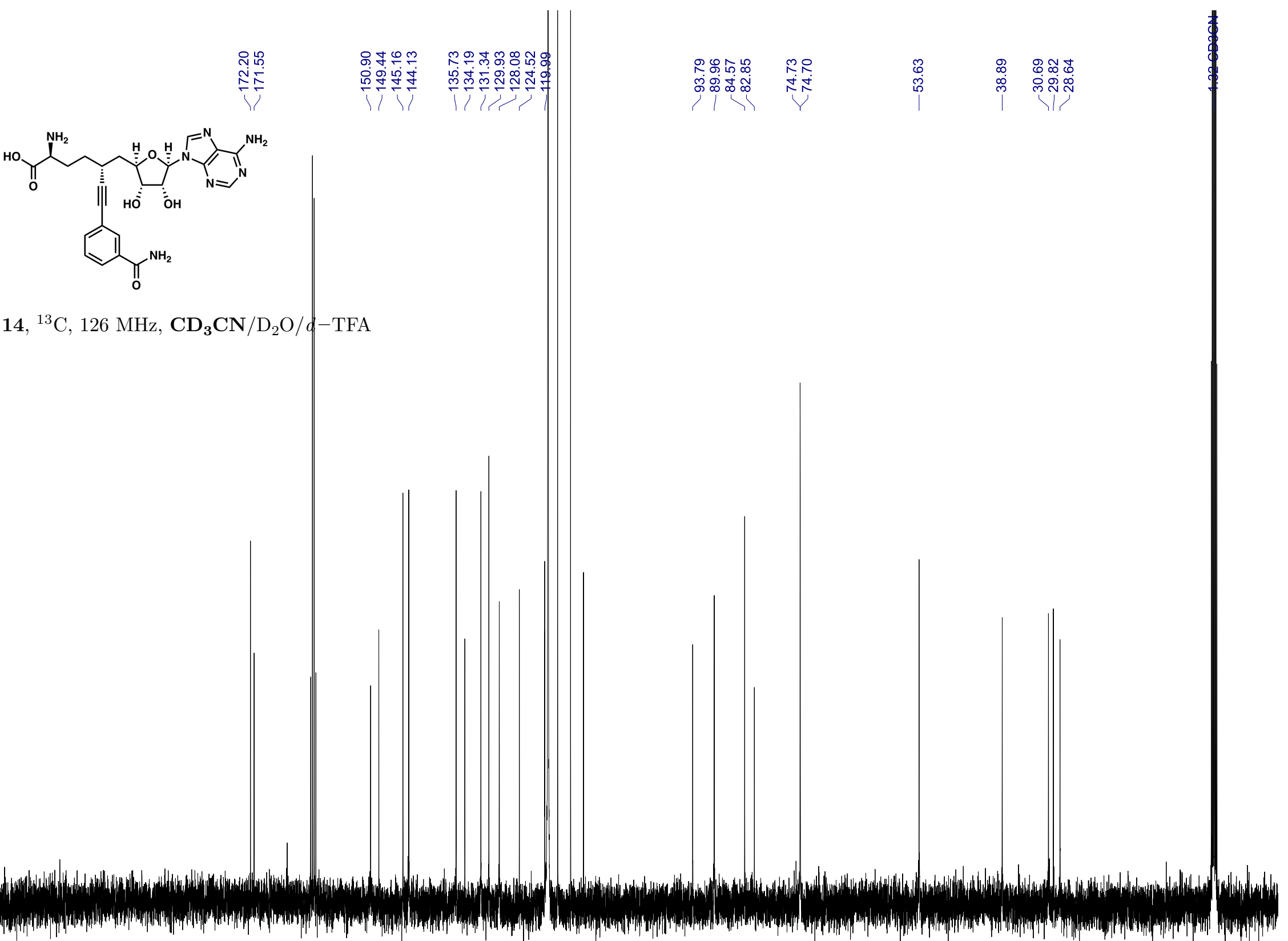

$\begin{array}{llllll}210 & 200 & 190 & 180 & 170 & 160\end{array}$

150

130

$120 \quad 110 \quad 100$

90

80

60

50

$40 \quad 30$

$20 \quad 10$

0 


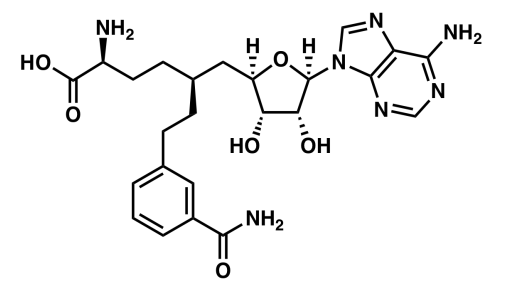

15, ${ }^{1} \mathrm{H}, 600 \mathrm{MHz}, \mathbf{C D}_{\mathbf{3}} \mathbf{C N} / \mathrm{D}_{2} \mathrm{O} / d-\mathrm{TFA}$

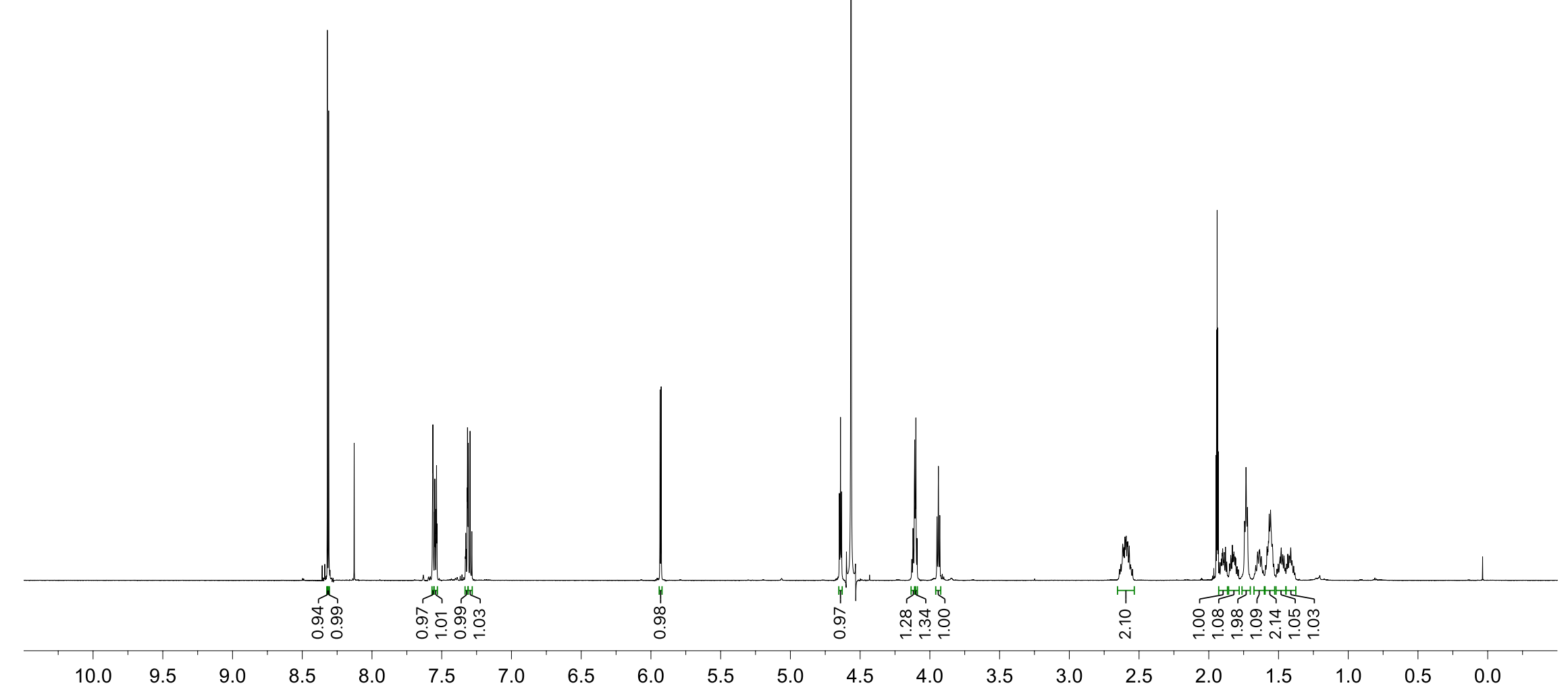




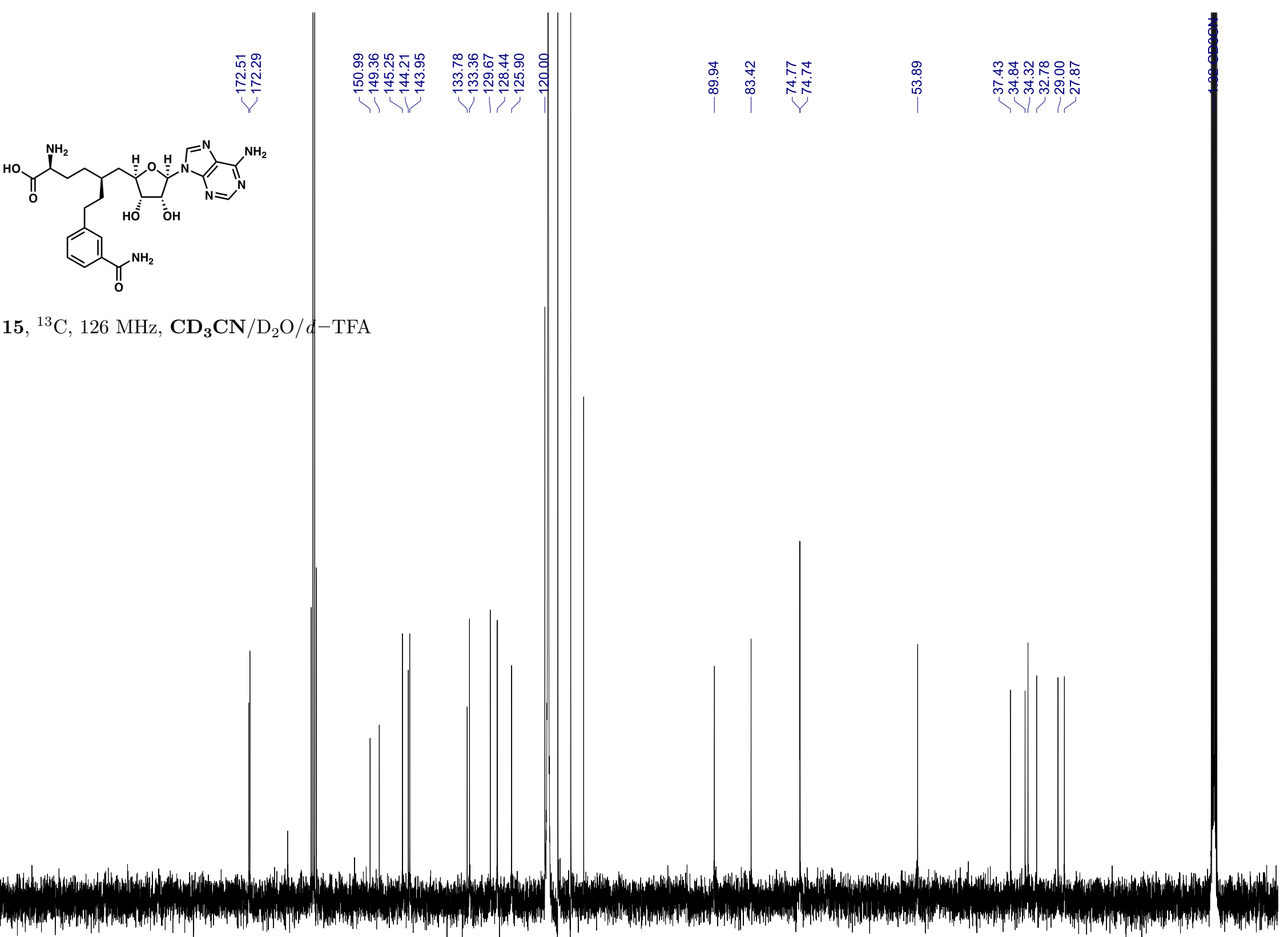

$210 \quad 200 \quad 190$

180

$170 \quad 160$

150

130

120

110

100

90

80

60

50

40

30

20

10 


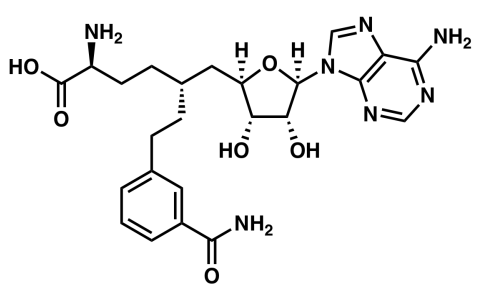

16, ${ }^{1} \mathrm{H}, 600 \mathrm{MHz}, \mathbf{C D}_{\mathbf{3}} \mathbf{C N} / \mathrm{D}_{2} \mathrm{O}$

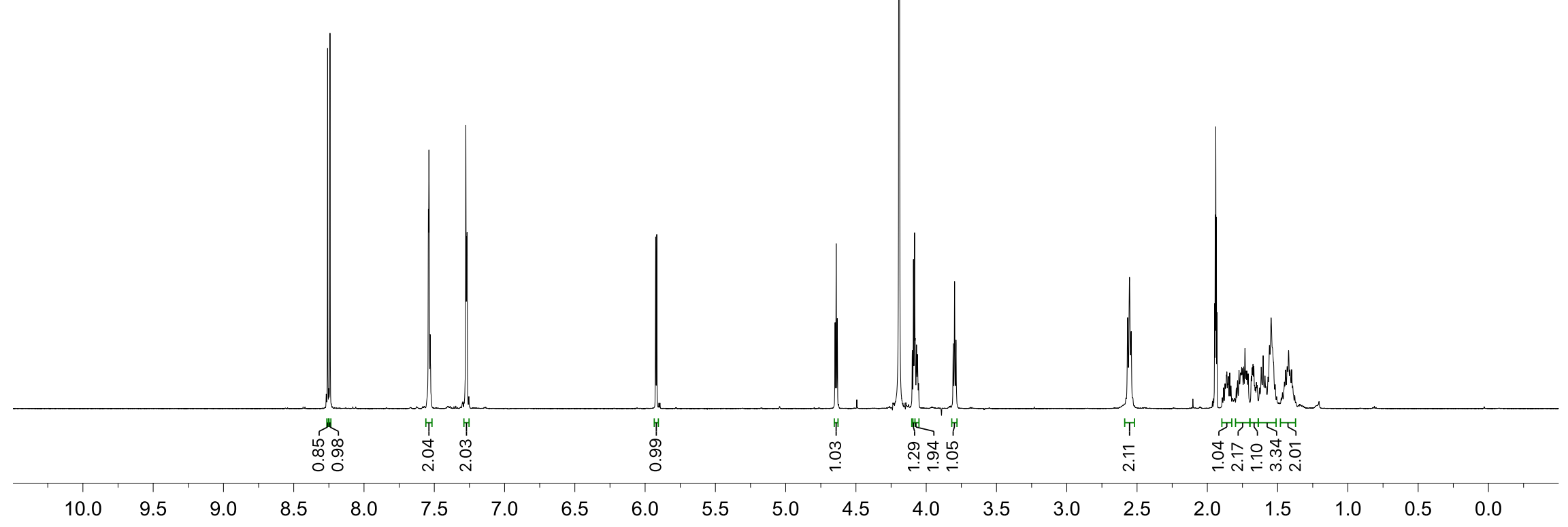




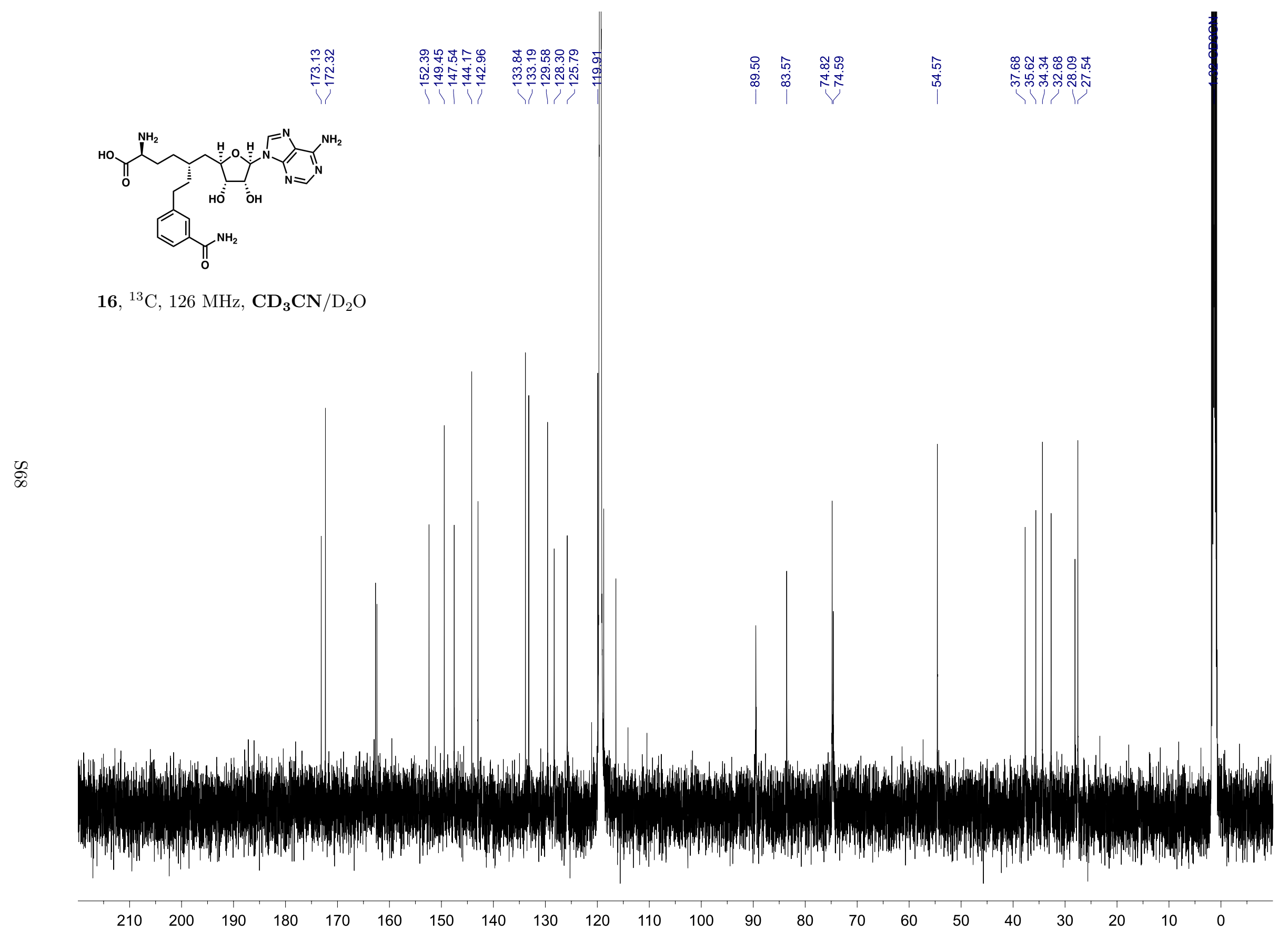




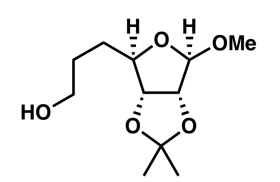

S21, ${ }^{1} \mathrm{H}, 600 \mathrm{MHz}, \mathrm{CDCl}_{3}$

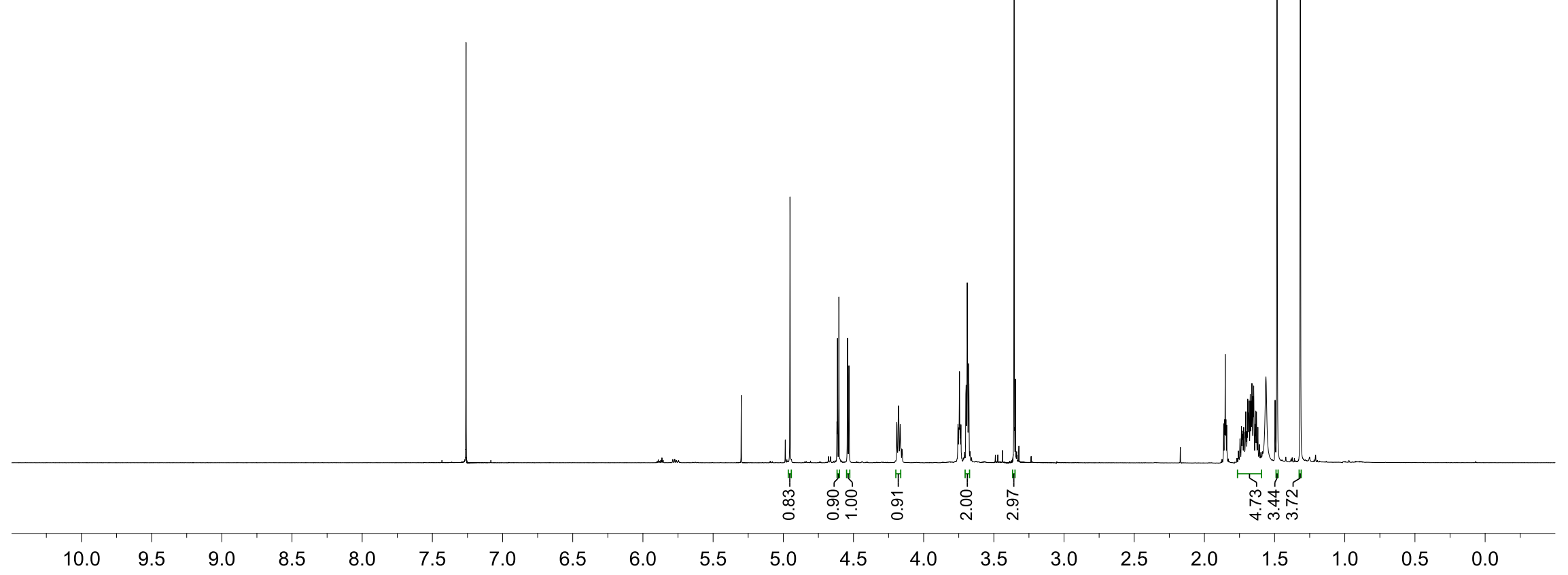




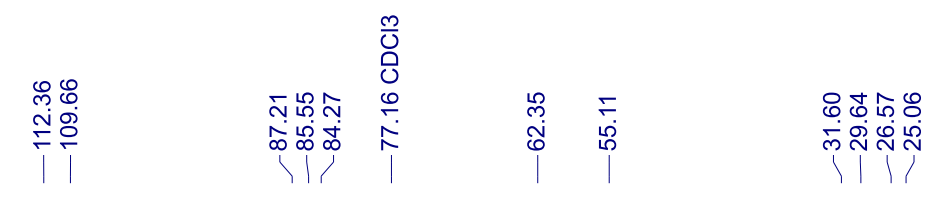

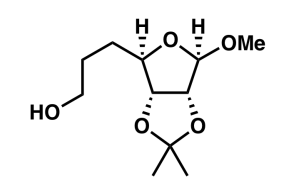

S21, ${ }^{13} \mathrm{C}, 151 \mathrm{MHz}, \mathrm{CDCl}_{3}$

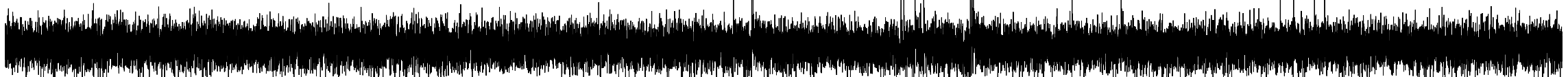




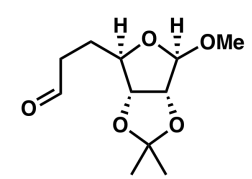

S22, ${ }^{1} \mathrm{H}, 500 \mathrm{MHz}, \mathrm{CDCl}_{3}$

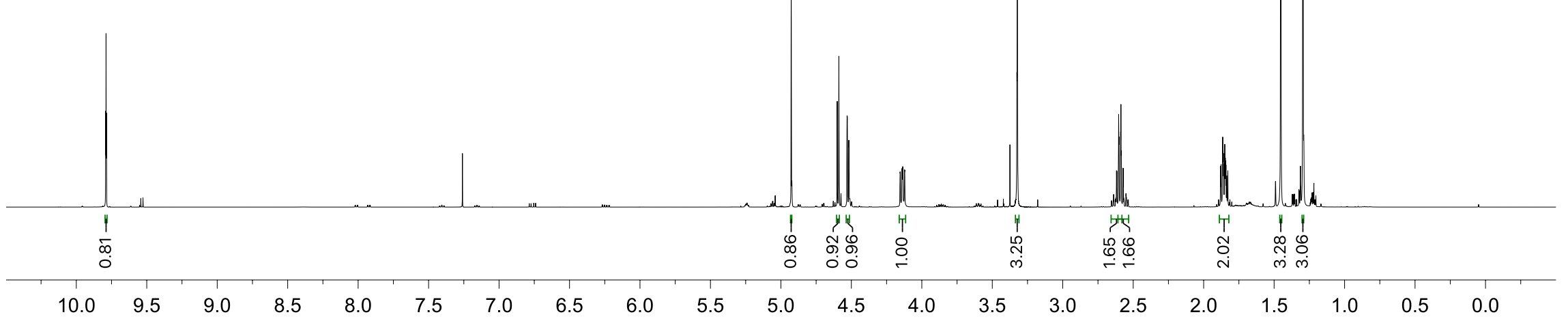




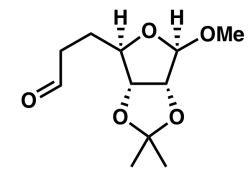

S22, ${ }^{13} \mathrm{C}, 151 \mathrm{MHz}, \mathrm{CDCl}_{3}$

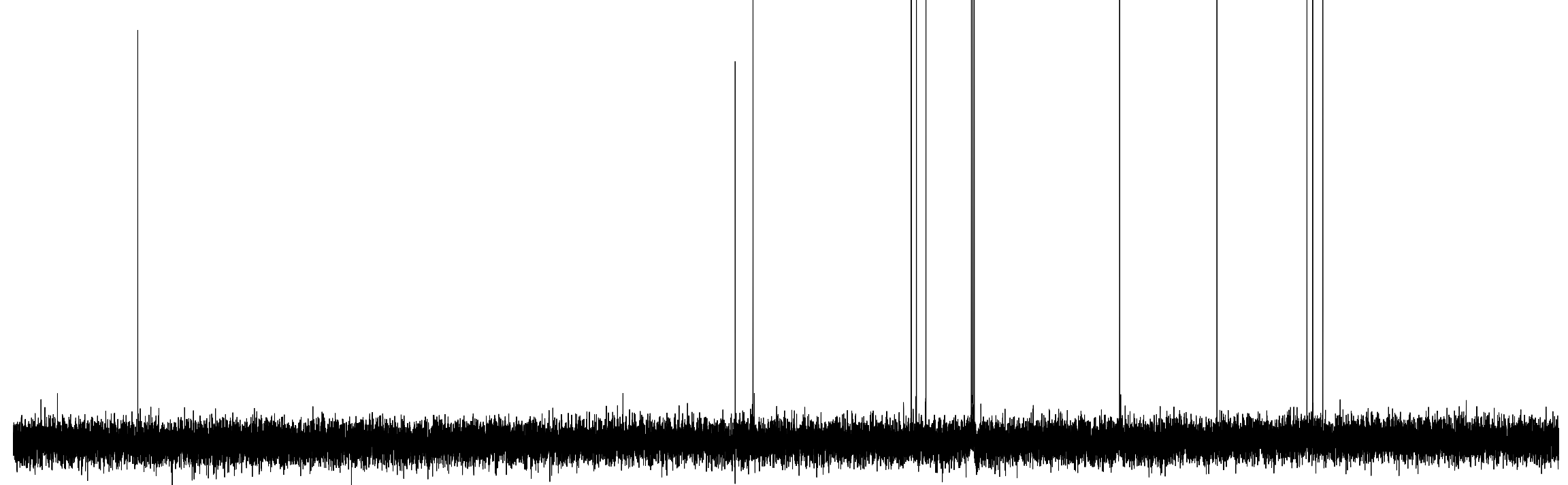




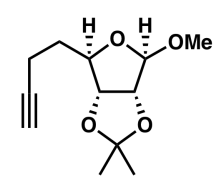

S23, ${ }^{1} \mathrm{H}, 600 \mathrm{MHz}, \mathrm{CDCl}_{3}$

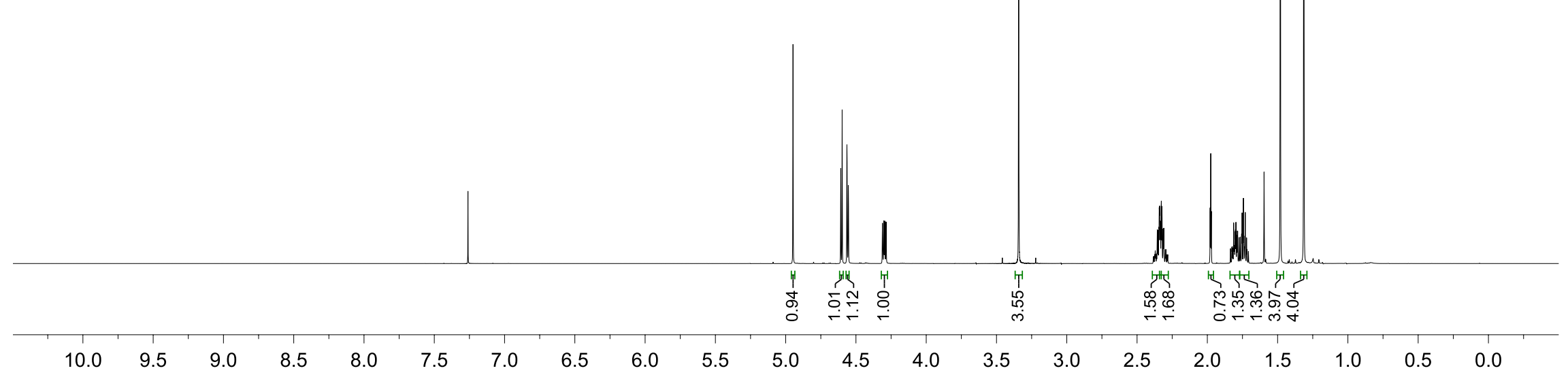




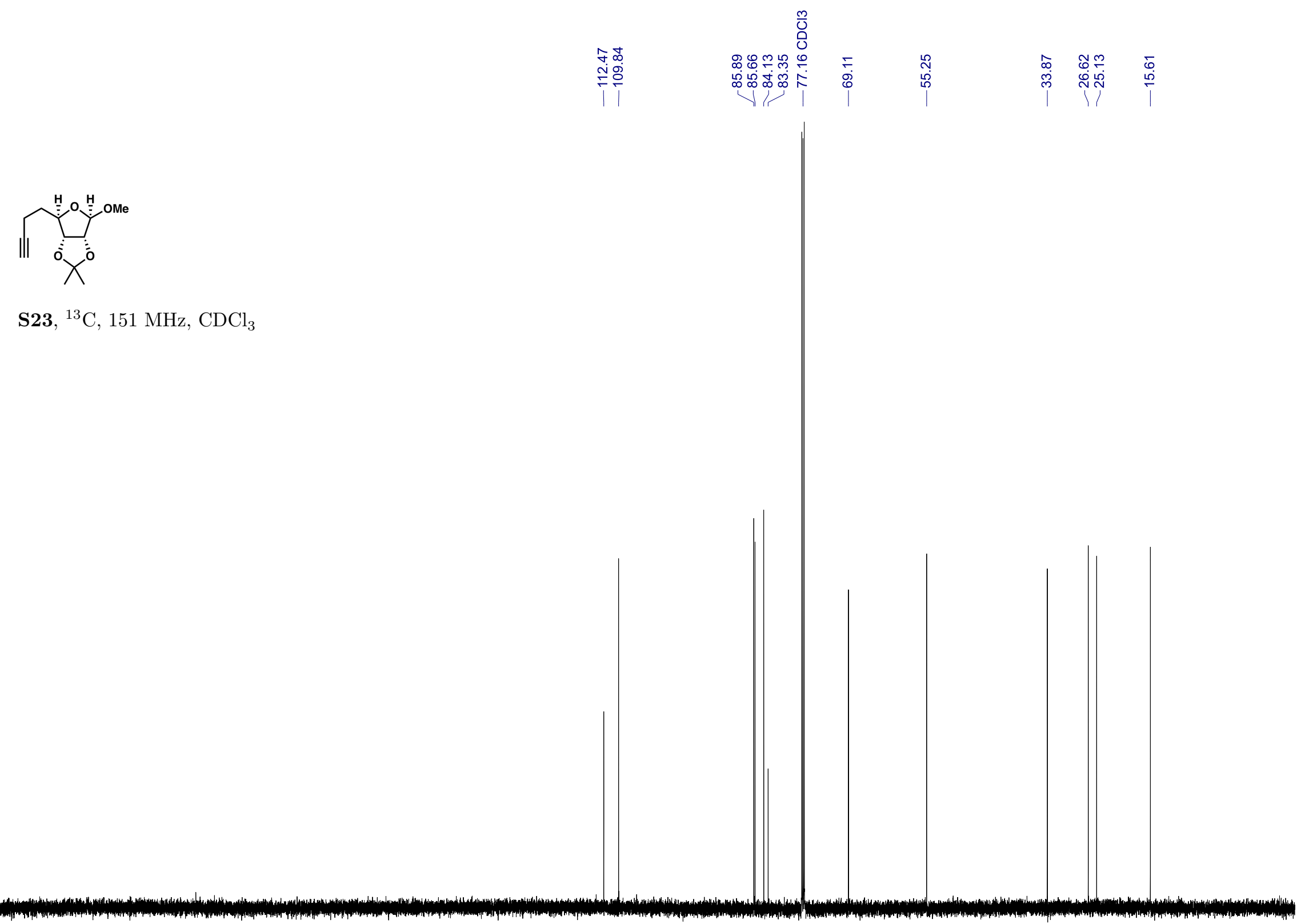




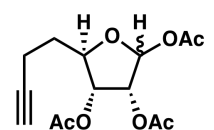

S24, ${ }^{1} \mathrm{H}, 600 \mathrm{MHz}, \mathrm{CDCl}_{3}$

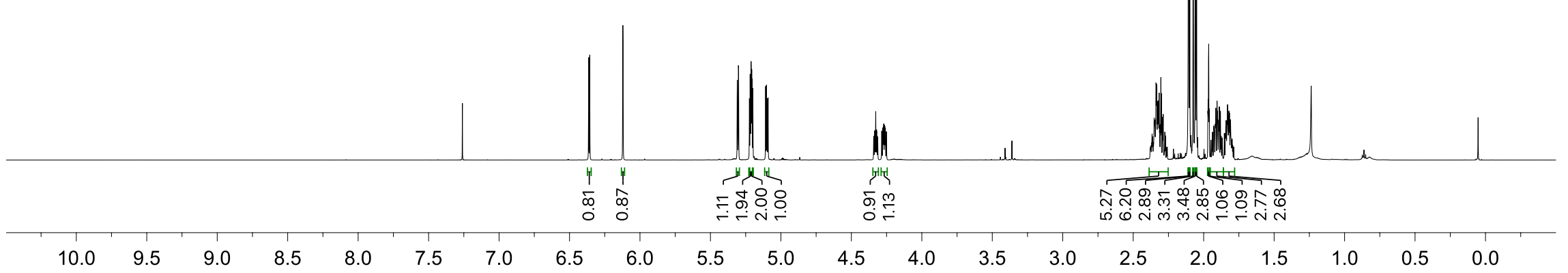


0.800 .87

영

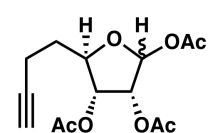

S24, ${ }^{13} \mathrm{C}, 126 \mathrm{MHz}, \mathrm{CDCl}_{3}$
근

के

| पी।

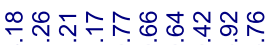

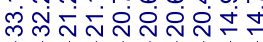

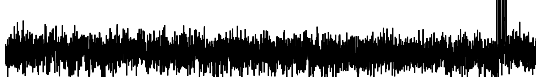

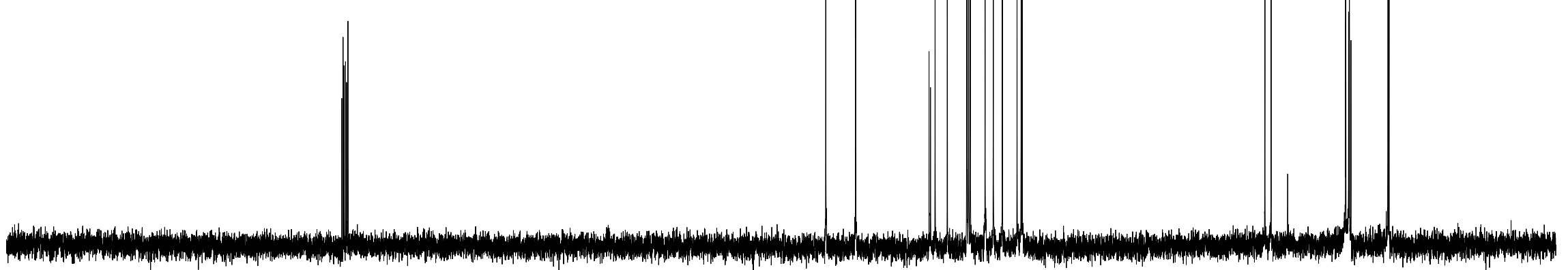




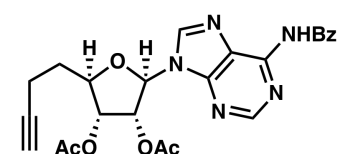

S25, ${ }^{1} \mathrm{H}, 600 \mathrm{MHz}, \mathrm{CDCl}_{3}$ 

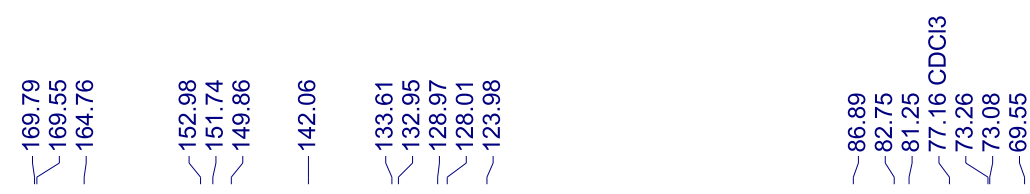

商

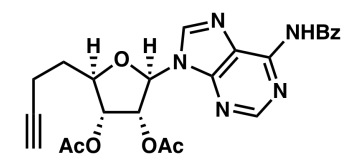

S25, ${ }^{13} \mathrm{C}, 151 \mathrm{MHz}, \mathrm{CDCl}_{3}$

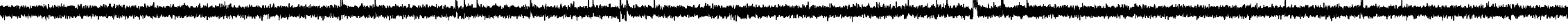




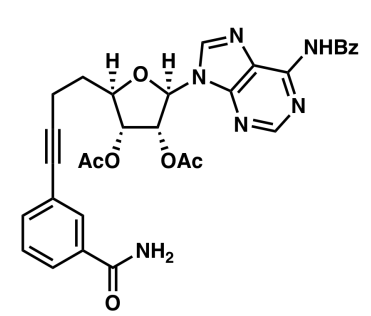

S26, ${ }^{1} \mathrm{H}, 600 \mathrm{MHz}, \mathrm{CDCl}_{3}$

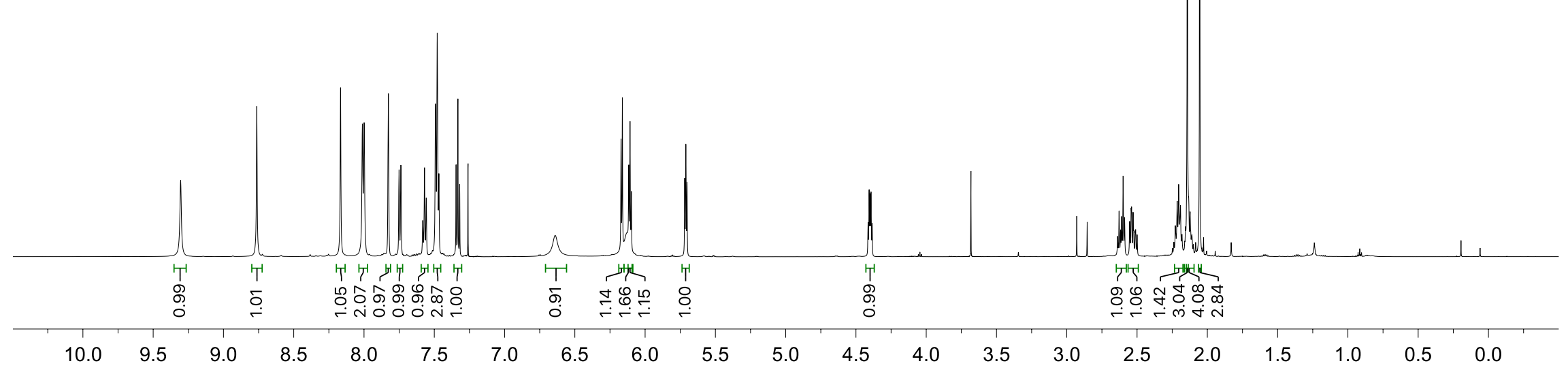




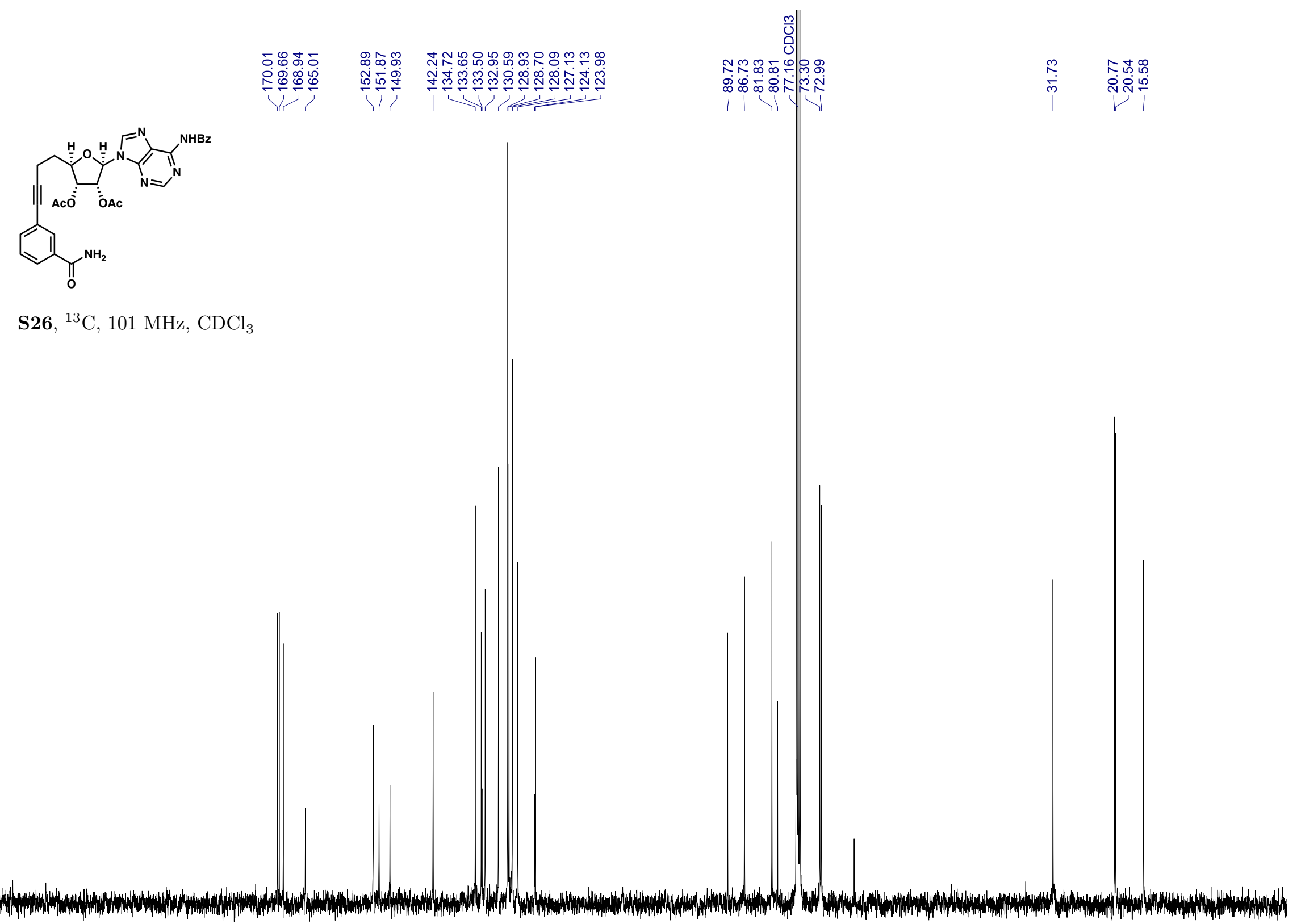

$210 \quad 200$

190

180

$170 \quad 160$

150

140

130

120

110

100

90

80

70

60

50

$40 \quad 30$

20 


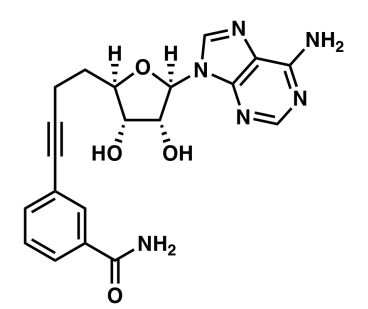

17, ${ }^{1} \mathrm{H}, 600 \mathrm{MHz}, \mathbf{C D}_{\mathbf{3}} \mathbf{C N} / \mathrm{D}_{2} \mathrm{O}$

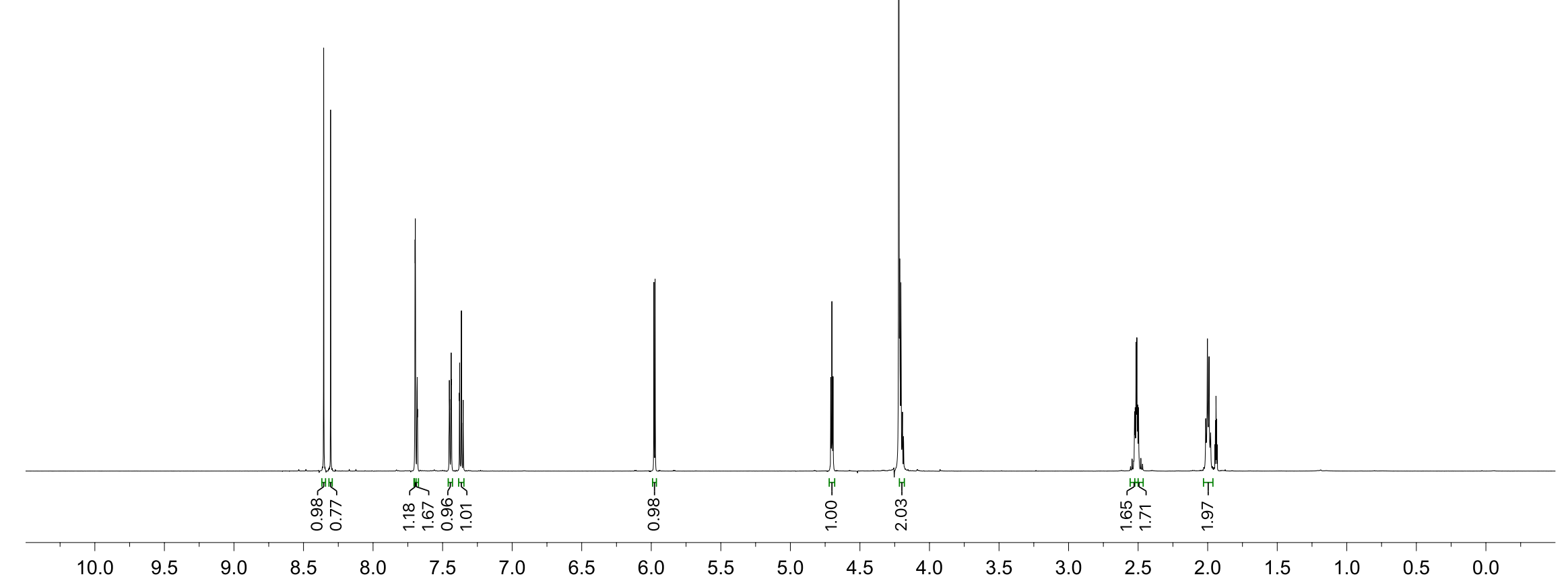



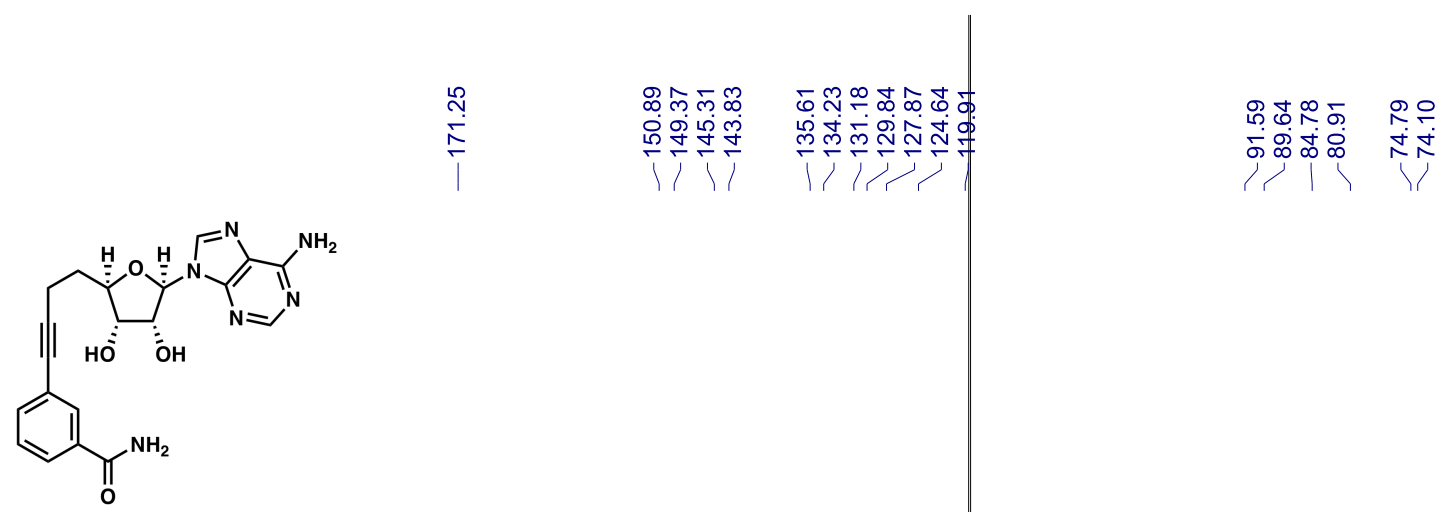

17, ${ }^{13} \mathrm{C}, 126 \mathrm{MHz}, \mathbf{C D}_{\mathbf{3}} \mathbf{C N} / \mathrm{D}_{2} \mathrm{O}$

D
$\infty$
$N$

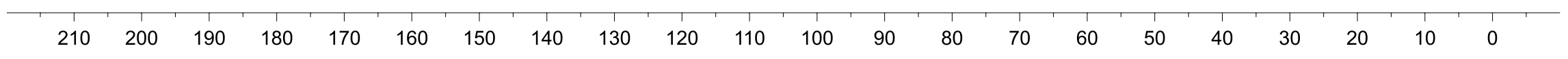




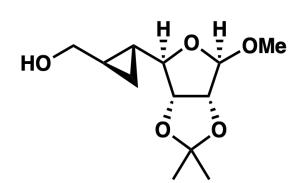

S28, ${ }^{1} \mathrm{H}, 600 \mathrm{MHz}, \mathrm{CDCl}_{3}$

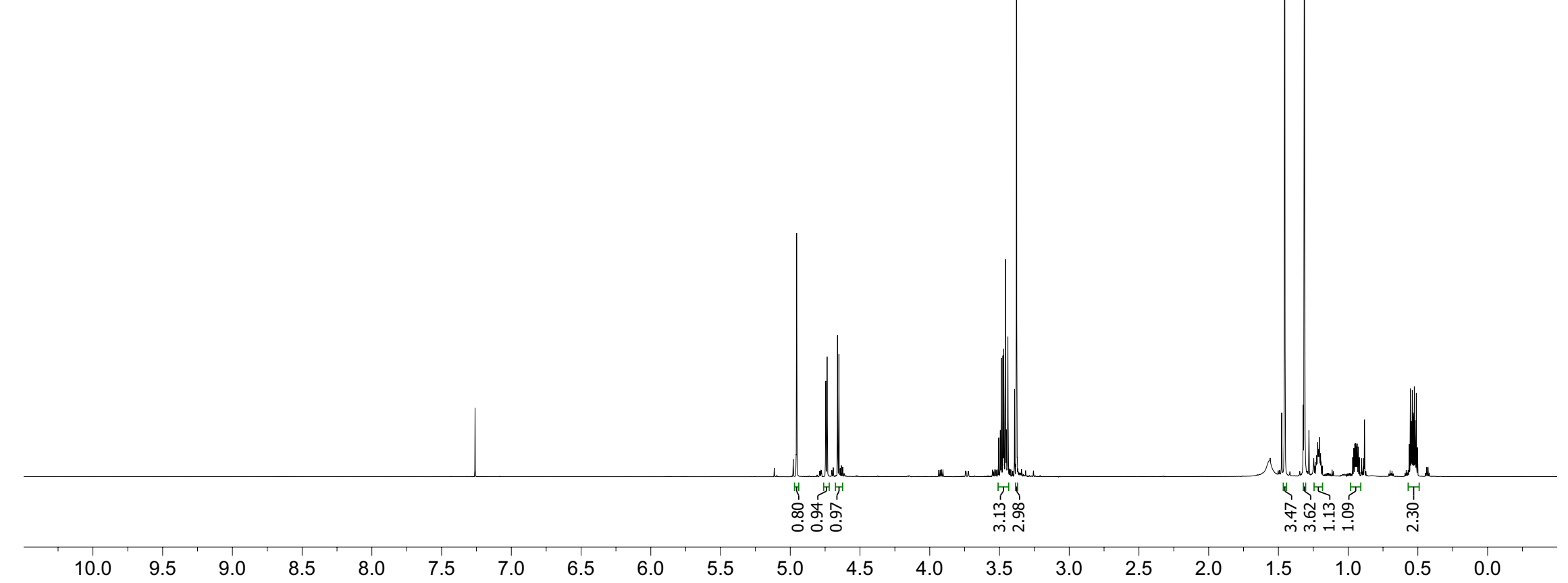




\begin{tabular}{|c|c|c|c|c|c|}
\hline | & 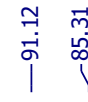 & 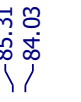 & $\begin{array}{l}0 \\
0 \\
0 \\
0 \\
0\end{array}$ & 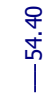 & 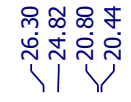 \\
\hline
\end{tabular}

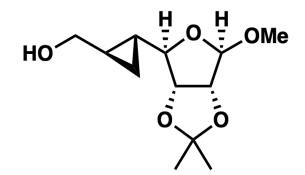

S28, ${ }^{13} \mathrm{C}, 151 \mathrm{MHz}, \mathrm{CDCl}_{3}$

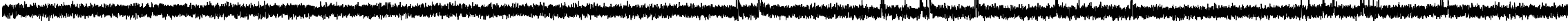

80

150

130

120

90

$80 \quad 70$

60

50

$40 \quad 30$ $20 \quad 10 \quad 0$ 


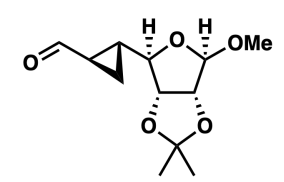

S29, ${ }^{1} \mathrm{H}, 600 \mathrm{MHz}, \mathrm{CDCl}_{3}$

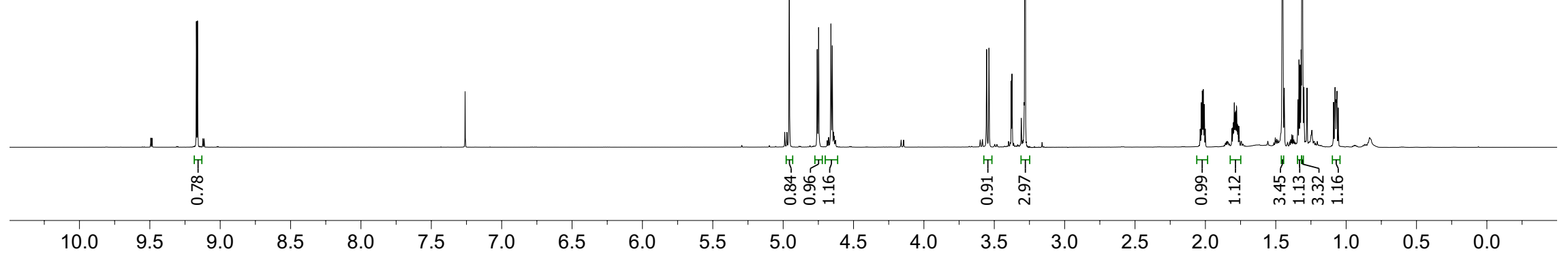




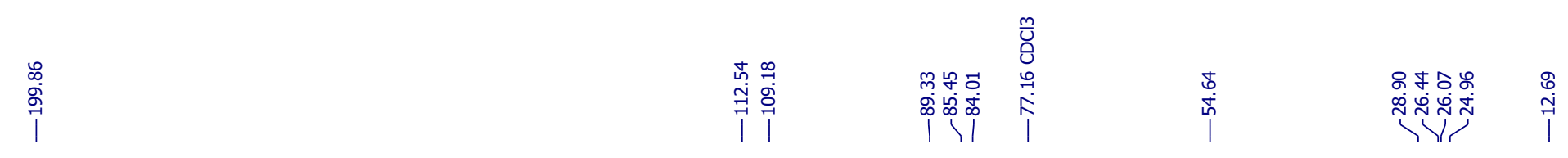

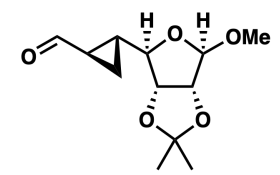

S29, ${ }^{13} \mathrm{C}, 151 \mathrm{MHz}, \mathrm{CDCl}_{3}$

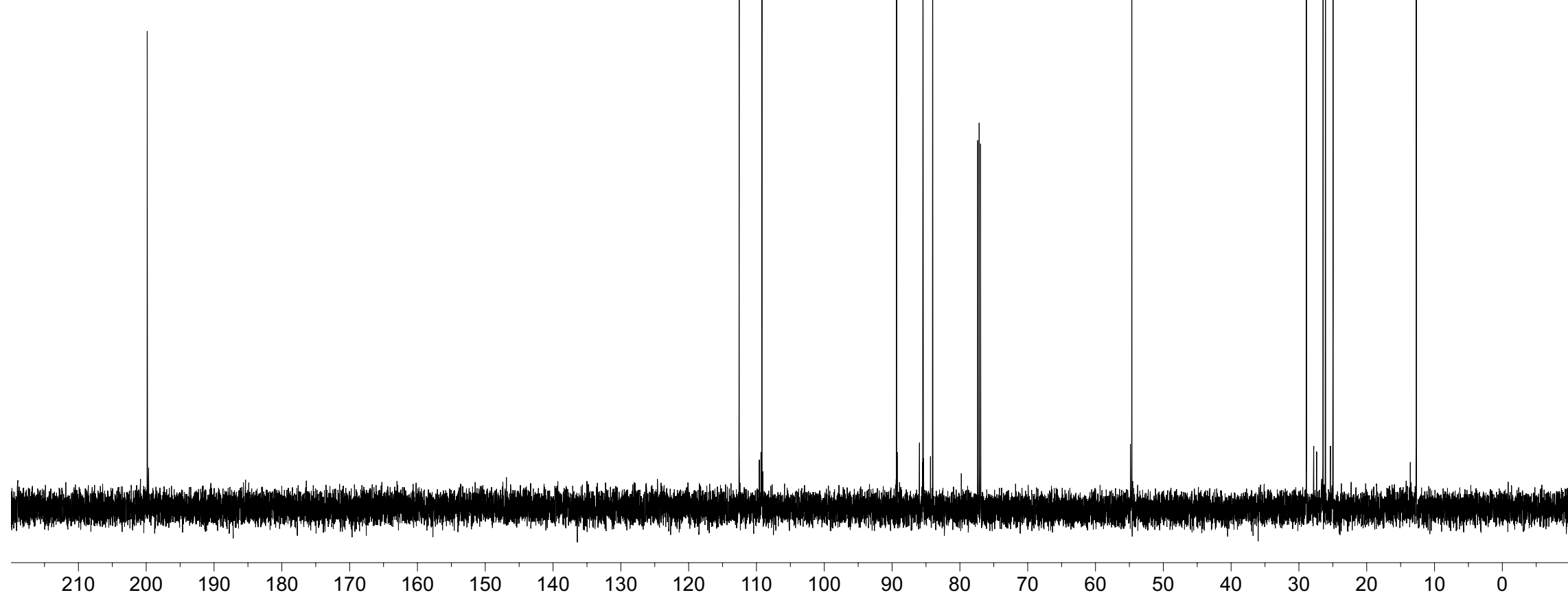




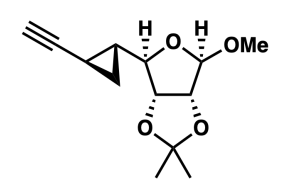

S30, ${ }^{1} \mathrm{H}, 600 \mathrm{MHz}, \mathrm{CDCl}_{3}$

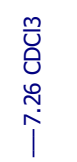

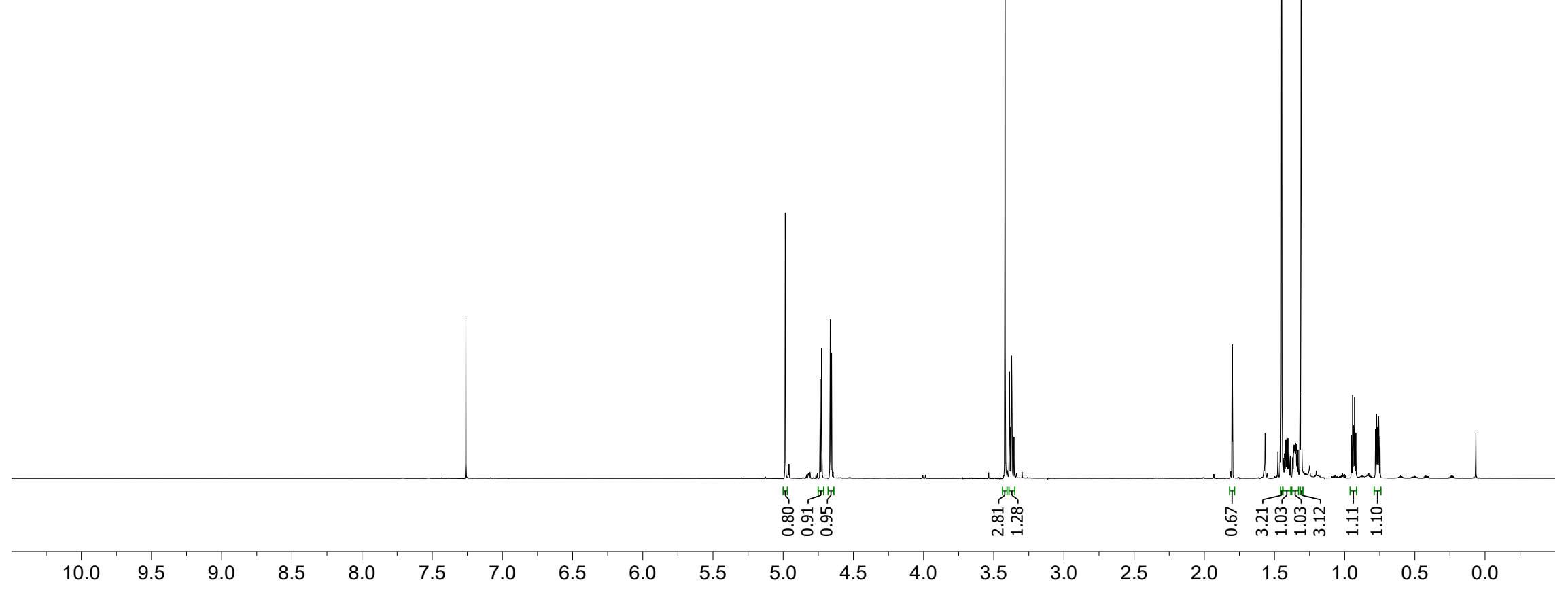




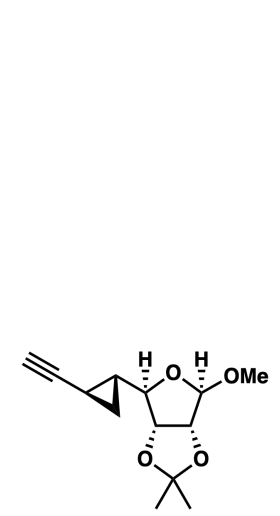

S30, ${ }^{13} \mathrm{C}, 151 \mathrm{MHz}, \mathrm{CDCl}_{3}$

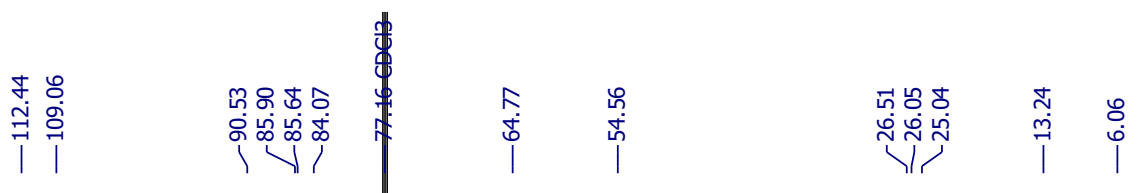

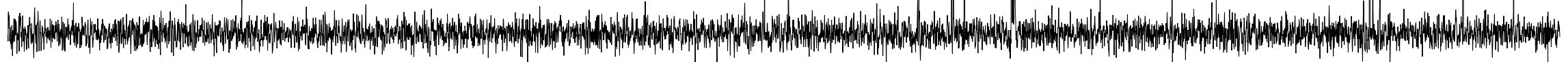

$210 \quad 200$

$170 \quad 160$

$150 \quad 140$

130

$20 \quad 110 \quad 100 \quad 90$

$80 \quad 70 \quad 60$

$\begin{array}{lll}50 & 40 \quad 30\end{array}$

$20 \quad 10 \quad 0$ 


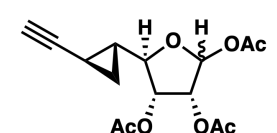

S31, ${ }^{1} \mathrm{H}, 600 \mathrm{MHz}, \mathrm{CDCl}_{3}$

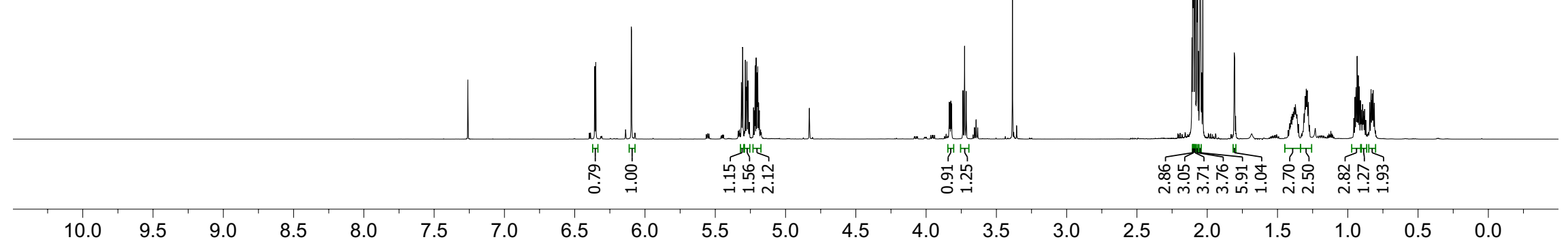




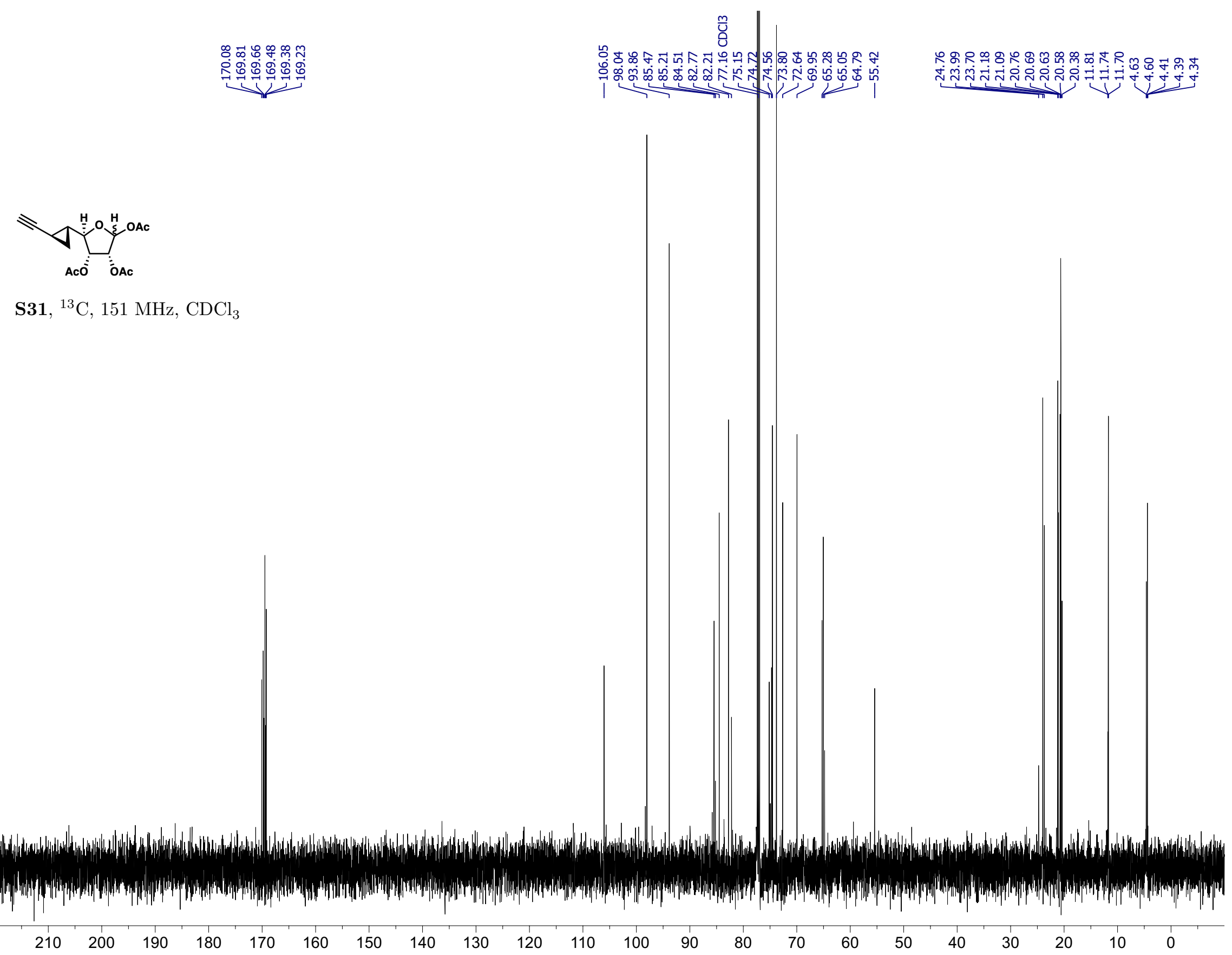




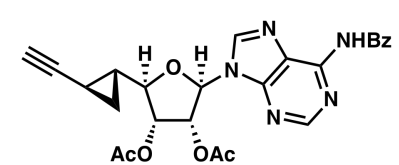

S32, ${ }^{1} \mathrm{H}, 500 \mathrm{MHz}, \mathrm{CDCl}_{3}$

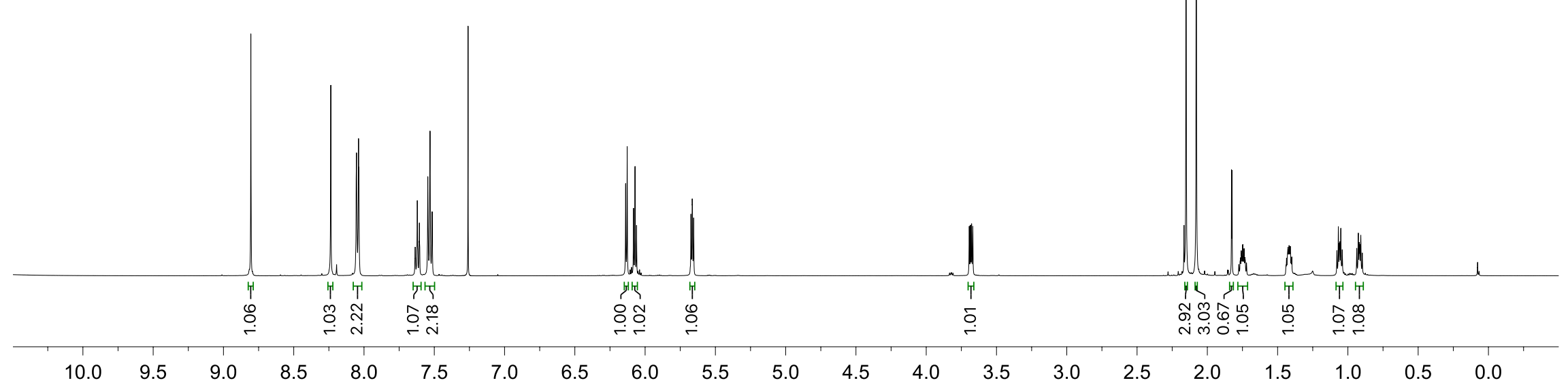



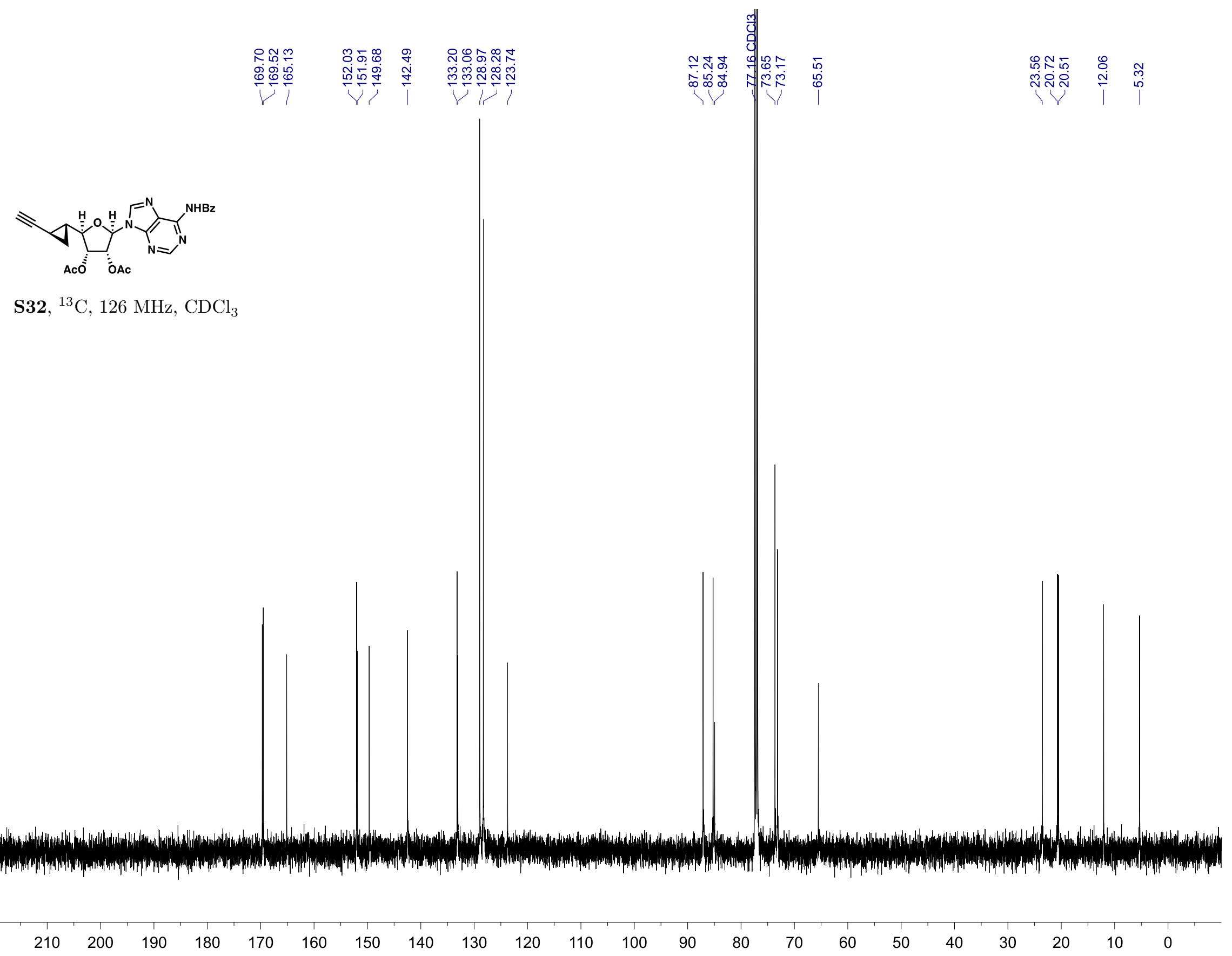


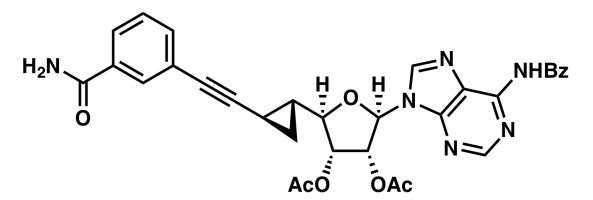

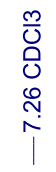

S33, ${ }^{1} \mathrm{H}, 500 \mathrm{MHz}, \mathrm{CDCl}_{3}$

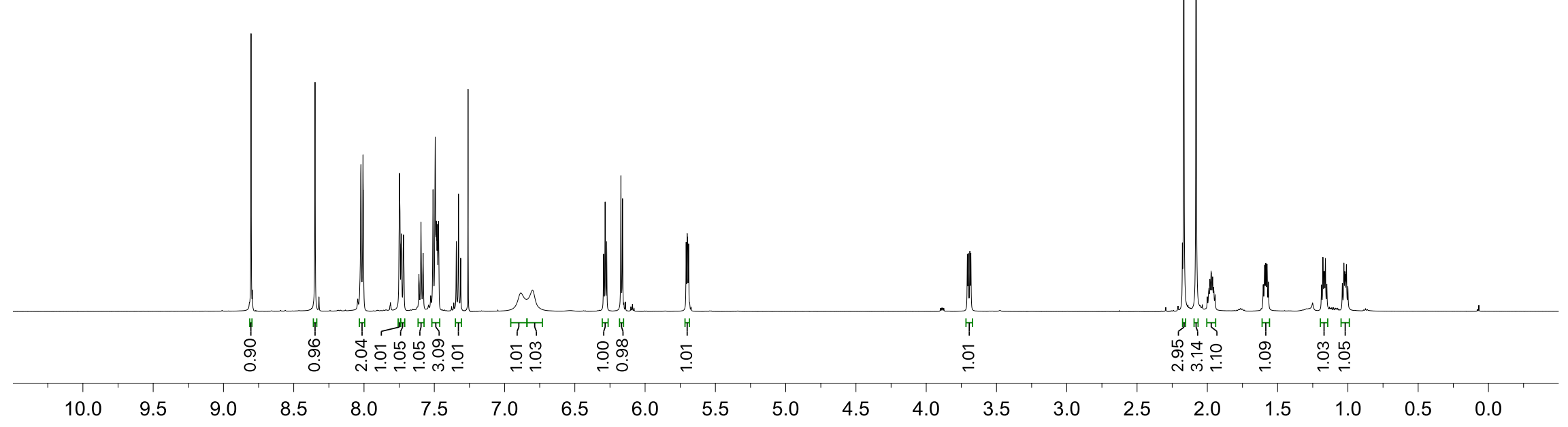



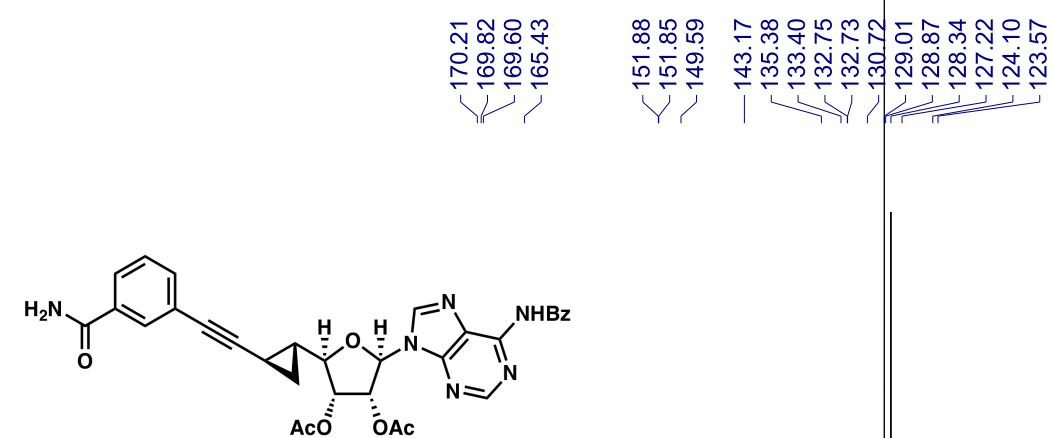

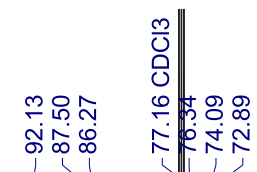

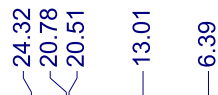

S33 ${ }^{13} \mathrm{C}, 126 \mathrm{MHz}, \mathrm{CDCl}_{3}$

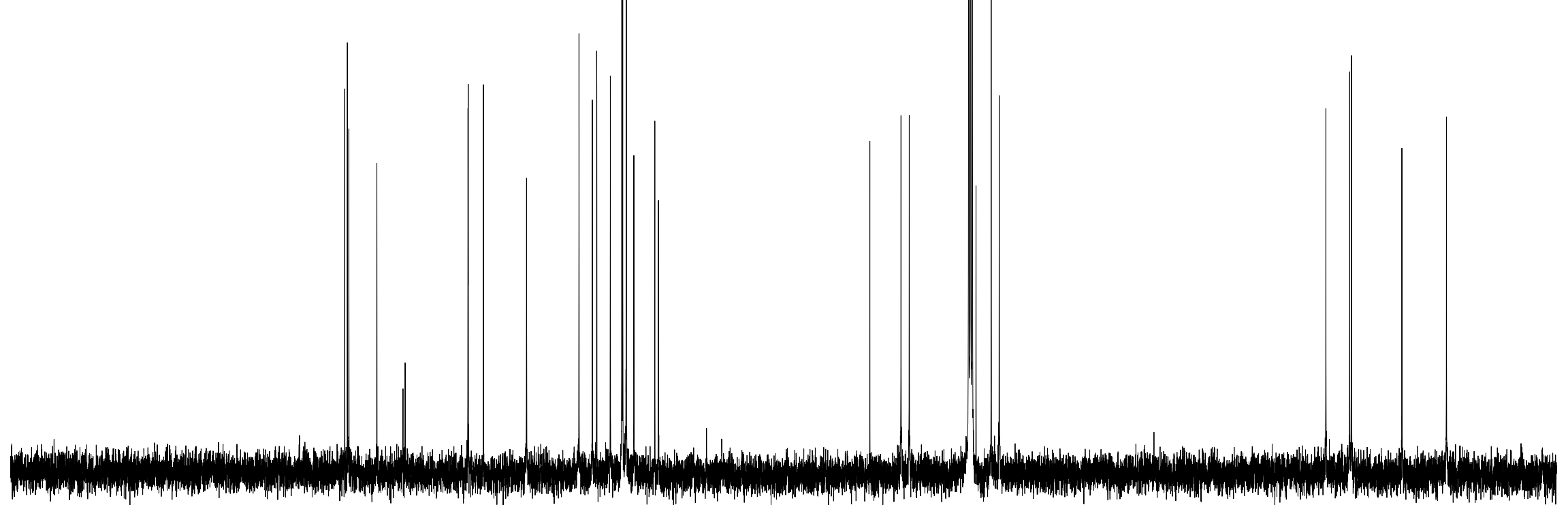




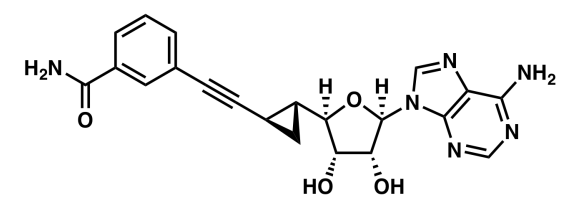

18, ${ }^{1} \mathrm{H}, 500 \mathrm{MHz}, \mathbf{C D}_{\mathbf{3}} \mathbf{C N} / \mathrm{D}_{2} \mathrm{O}$

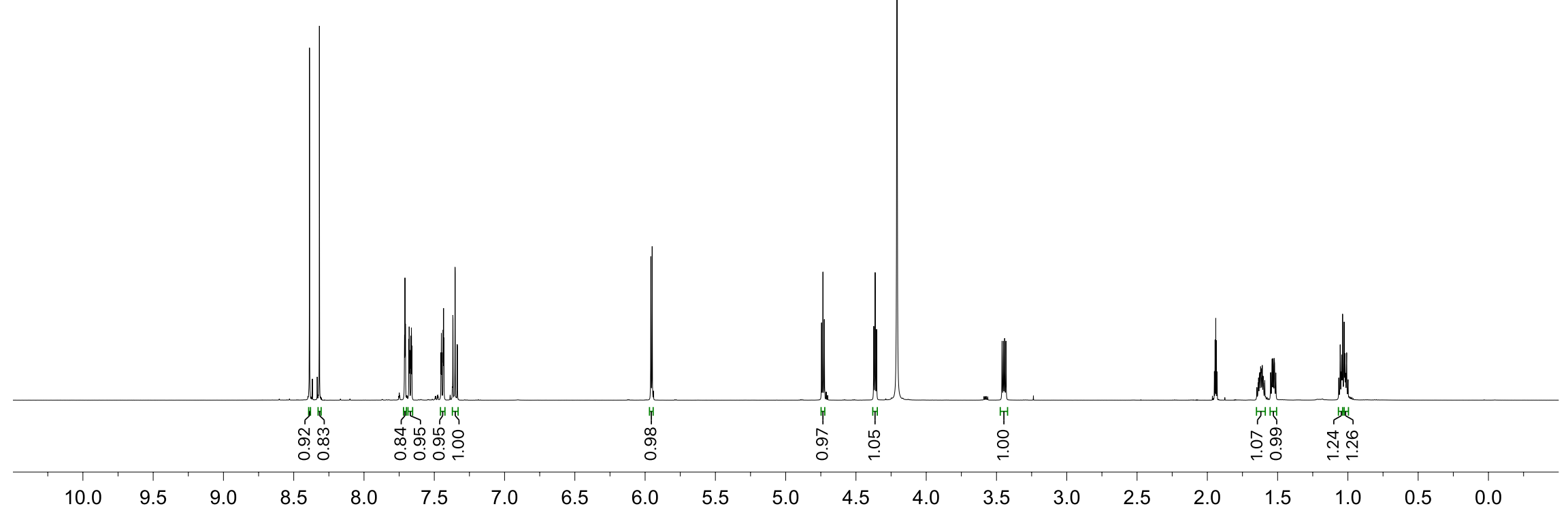




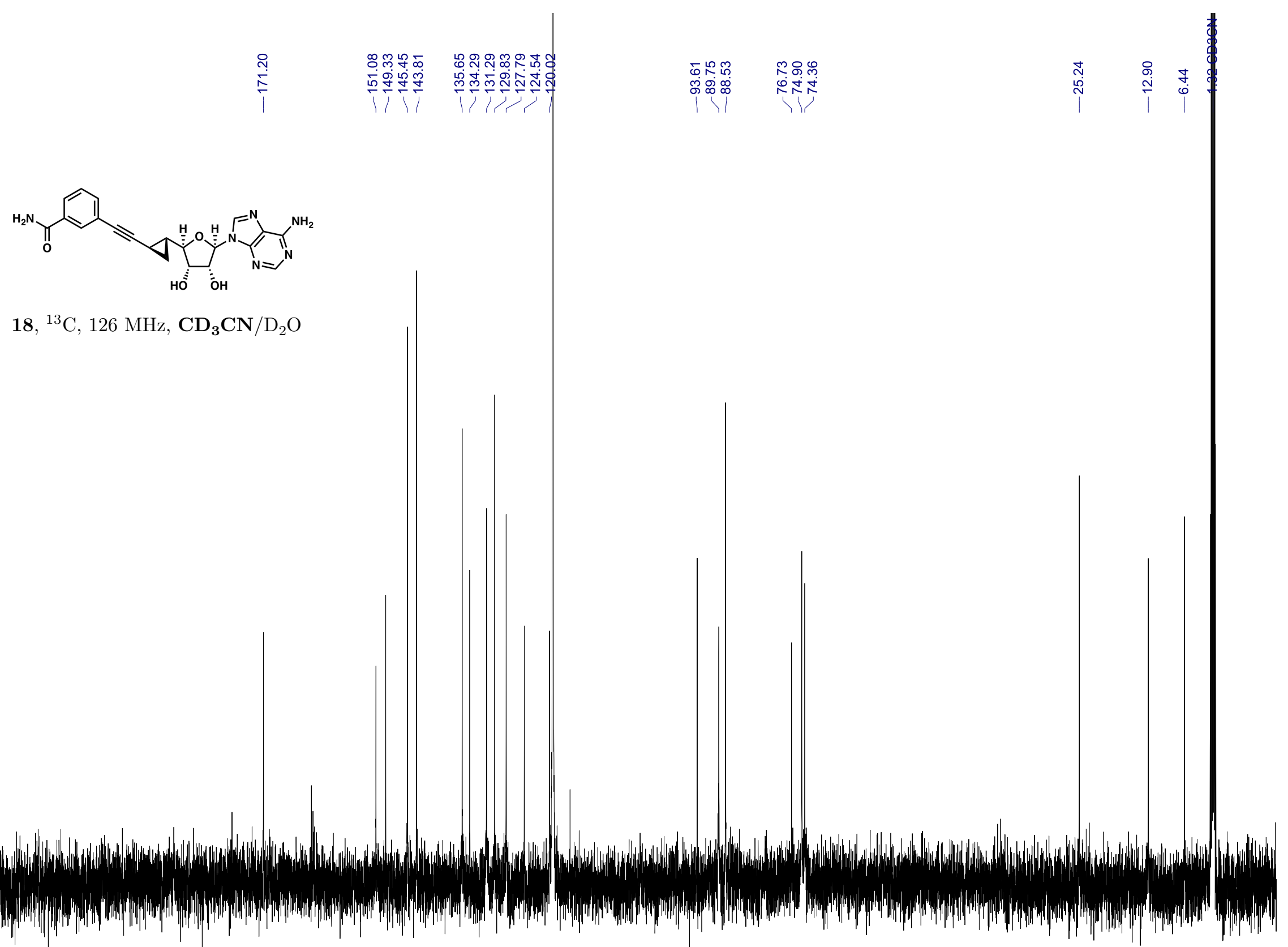

130

$120 \quad 110 \quad 100$

90

$80 \quad 70$

60

50

$40 \quad 30$

20

10

0 


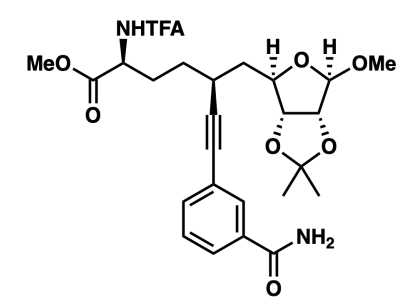

S34, ${ }^{1} \mathrm{H}, 500 \mathrm{MHz}, \mathrm{CDCl}_{3}$

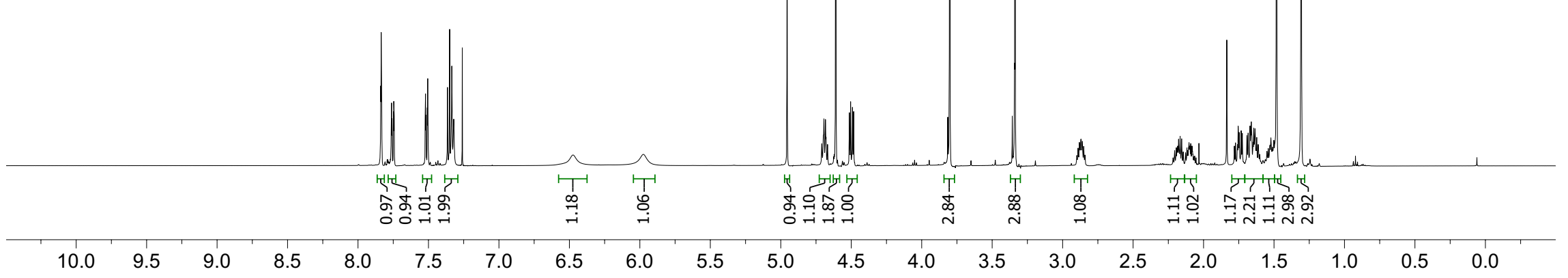




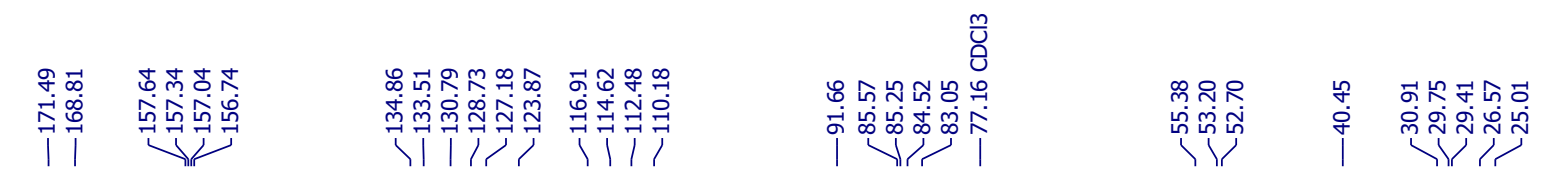

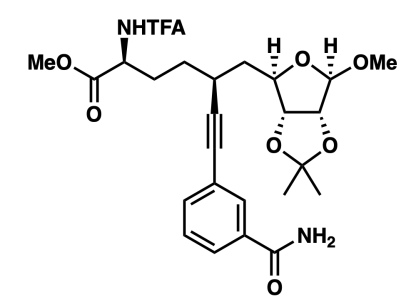

S34, ${ }^{13} \mathrm{C}, 126 \mathrm{MHz}, \mathrm{CDCl}_{3}$

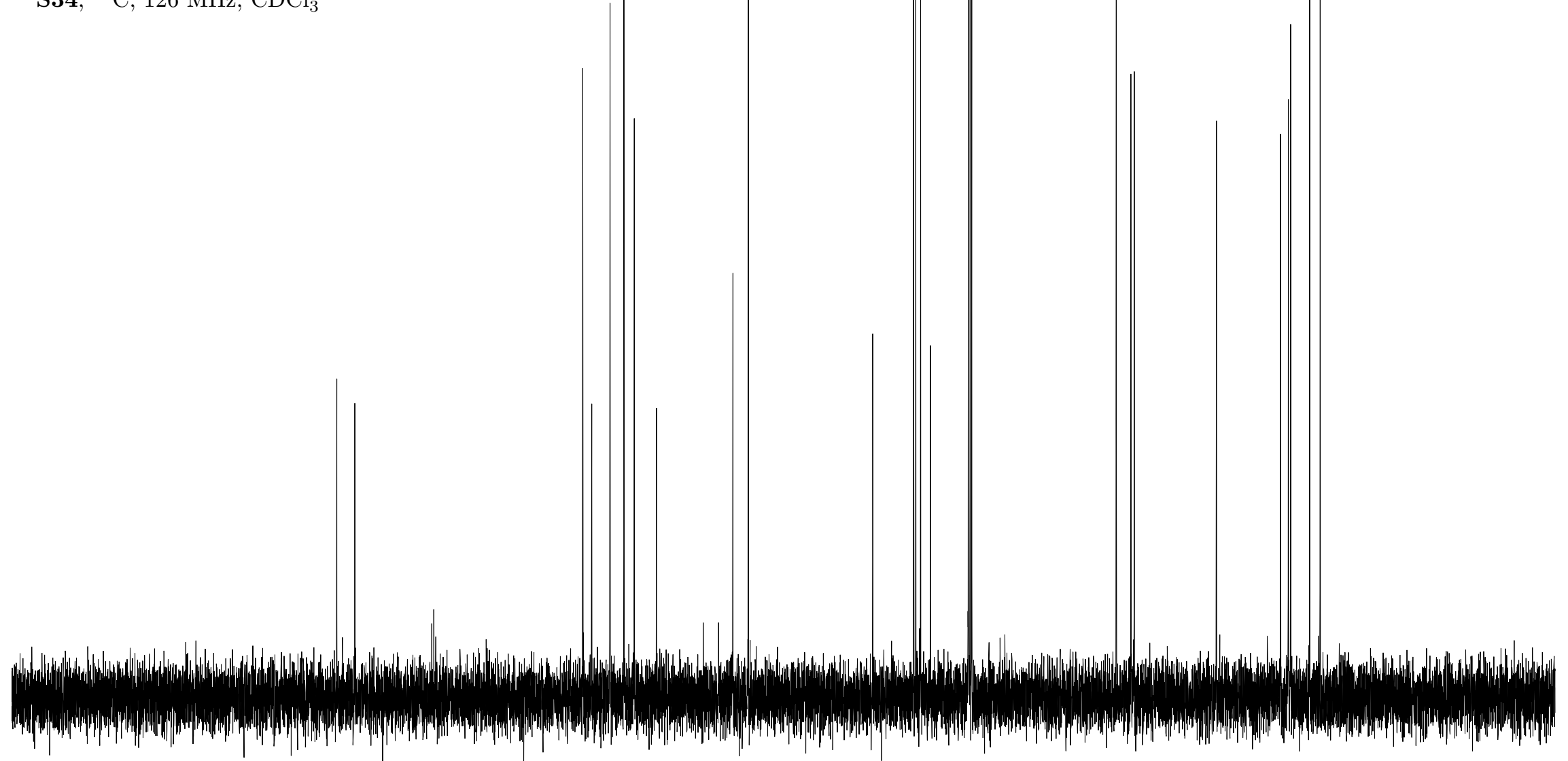

210200

$190 \quad 180$

170160

$150 \quad 140$

$130 \quad 120$

$110 \quad 100$

90

$80 \quad 70$

$60 \quad 50$

$40 \quad 30$

$20 \quad 10$ 


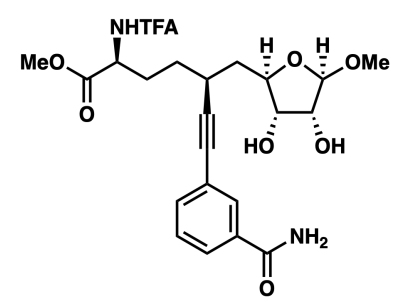

S35, ${ }^{1} \mathrm{H}, 600 \mathrm{MHz},\left(\mathrm{CD}_{3}\right)_{2} \mathrm{CO}$

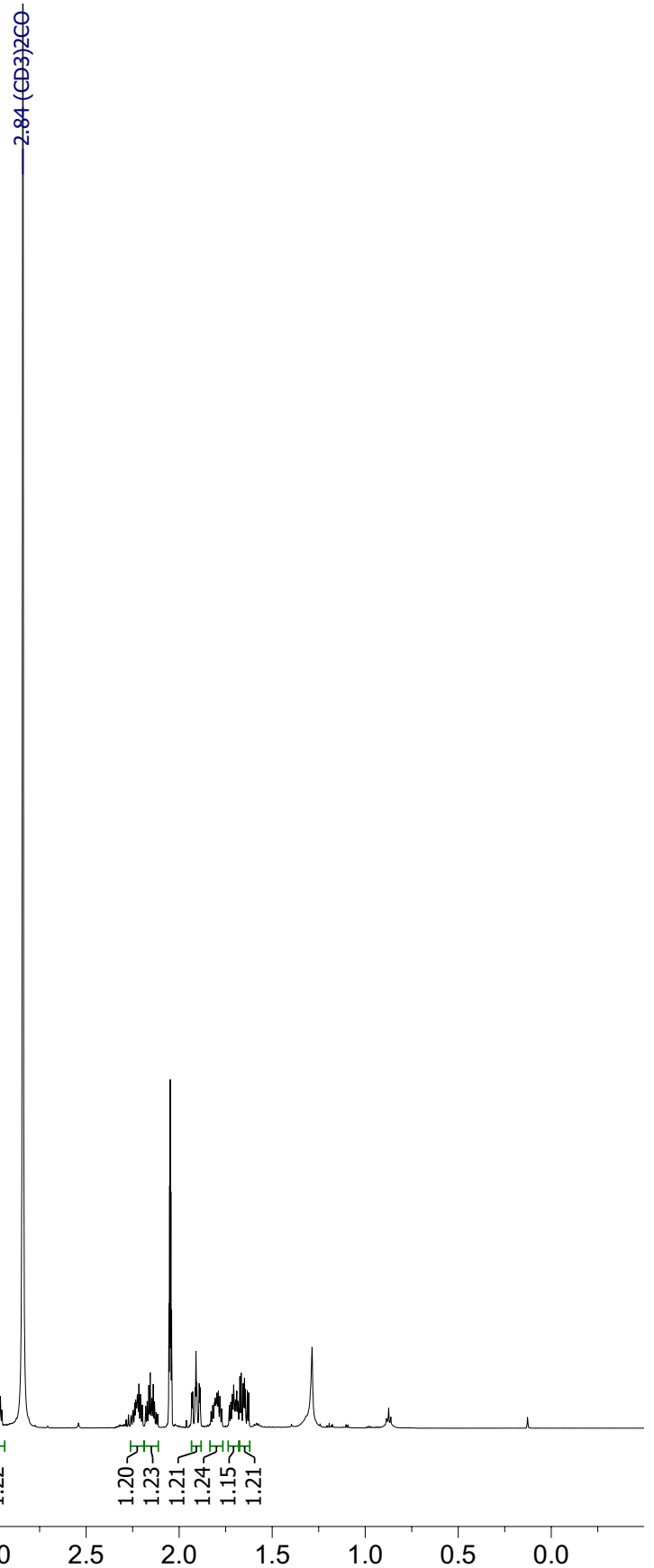




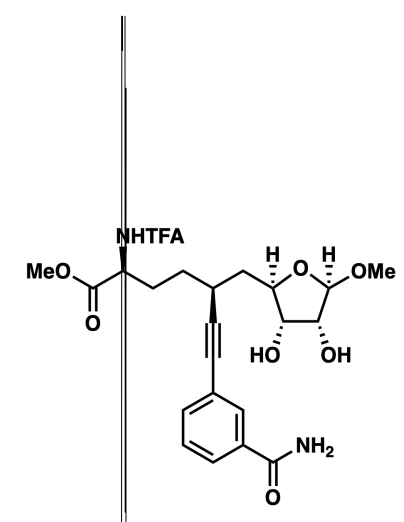

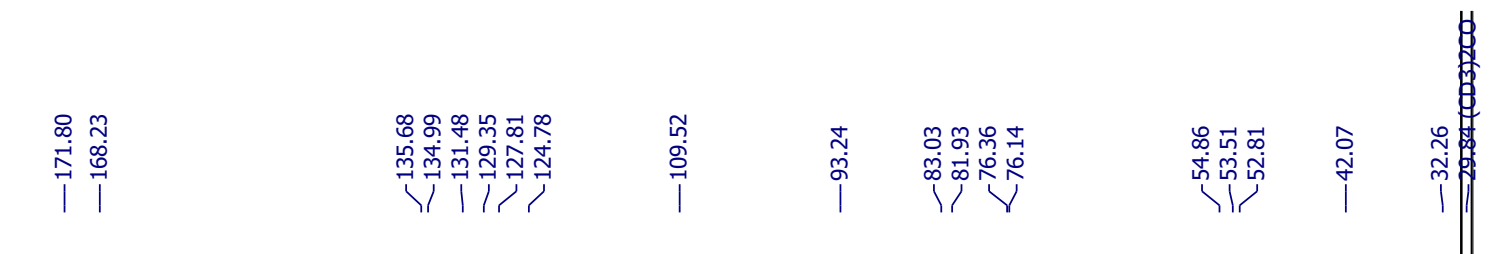

S35, ${ }^{13} \mathrm{C}, 101 \mathrm{MHz},\left(\mathrm{CD}_{3}\right)_{2} \mathrm{CO}$

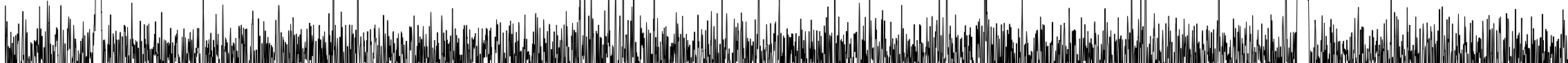

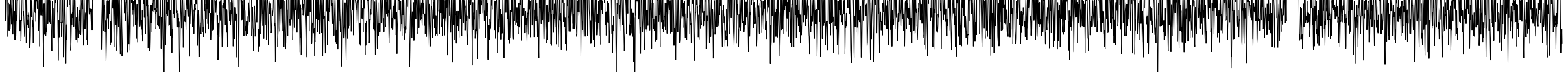

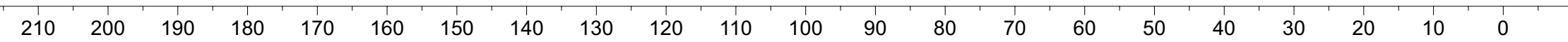




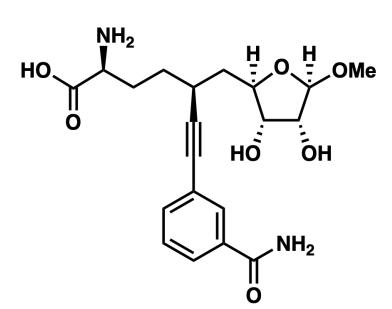

19, ${ }^{1} \mathrm{H}, 600 \mathrm{MHz}, \mathrm{D}_{2} \mathrm{O}$

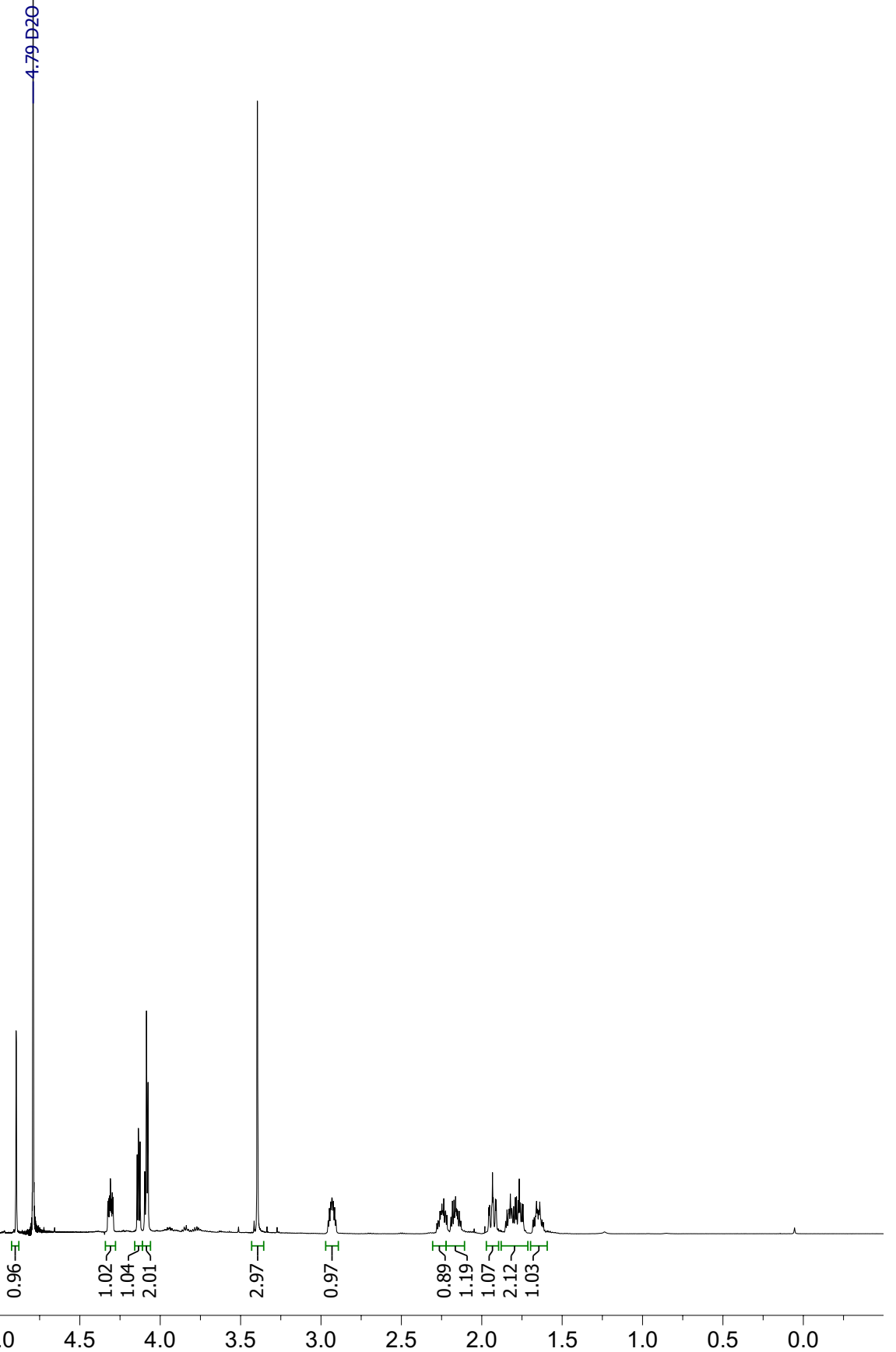




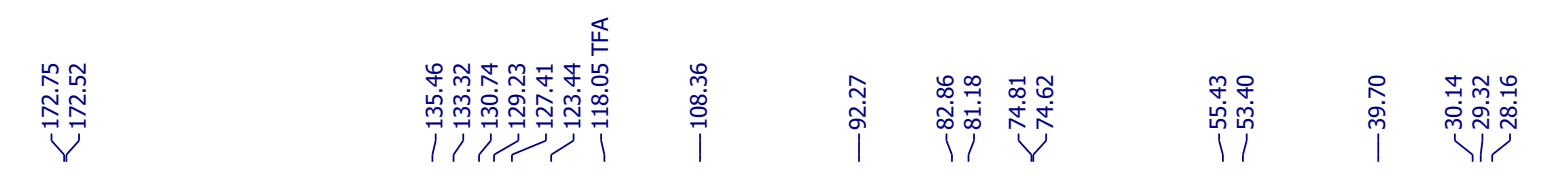

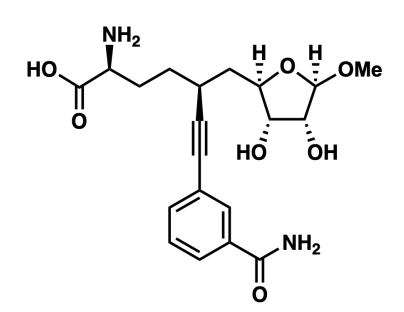

19, ${ }^{13} \mathrm{C}, 101 \mathrm{MHz}, \mathrm{D}_{2} \mathrm{O}$

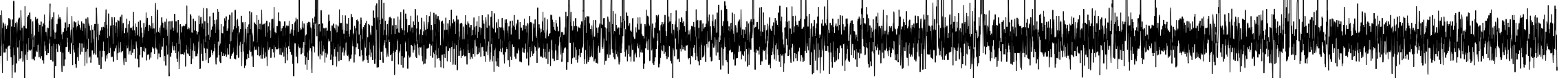

$\begin{array}{lllllllllllll}210 & 200 & 190 & 180 & 170 & 160 & 150 & 140 & 130 & 120 & 110 & 100 & 90\end{array}$

$80 \quad 70$

$60 \quad 50$

$40 \quad 30$

$20 \quad 10 \quad 0$ 


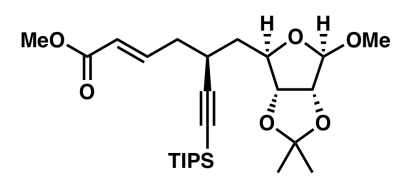

$\frac{m}{0}$
0
0
0
$i$

S36, ${ }^{1} \mathrm{H}, 600 \mathrm{MHz}, \mathrm{CDCl}_{3}$

莒

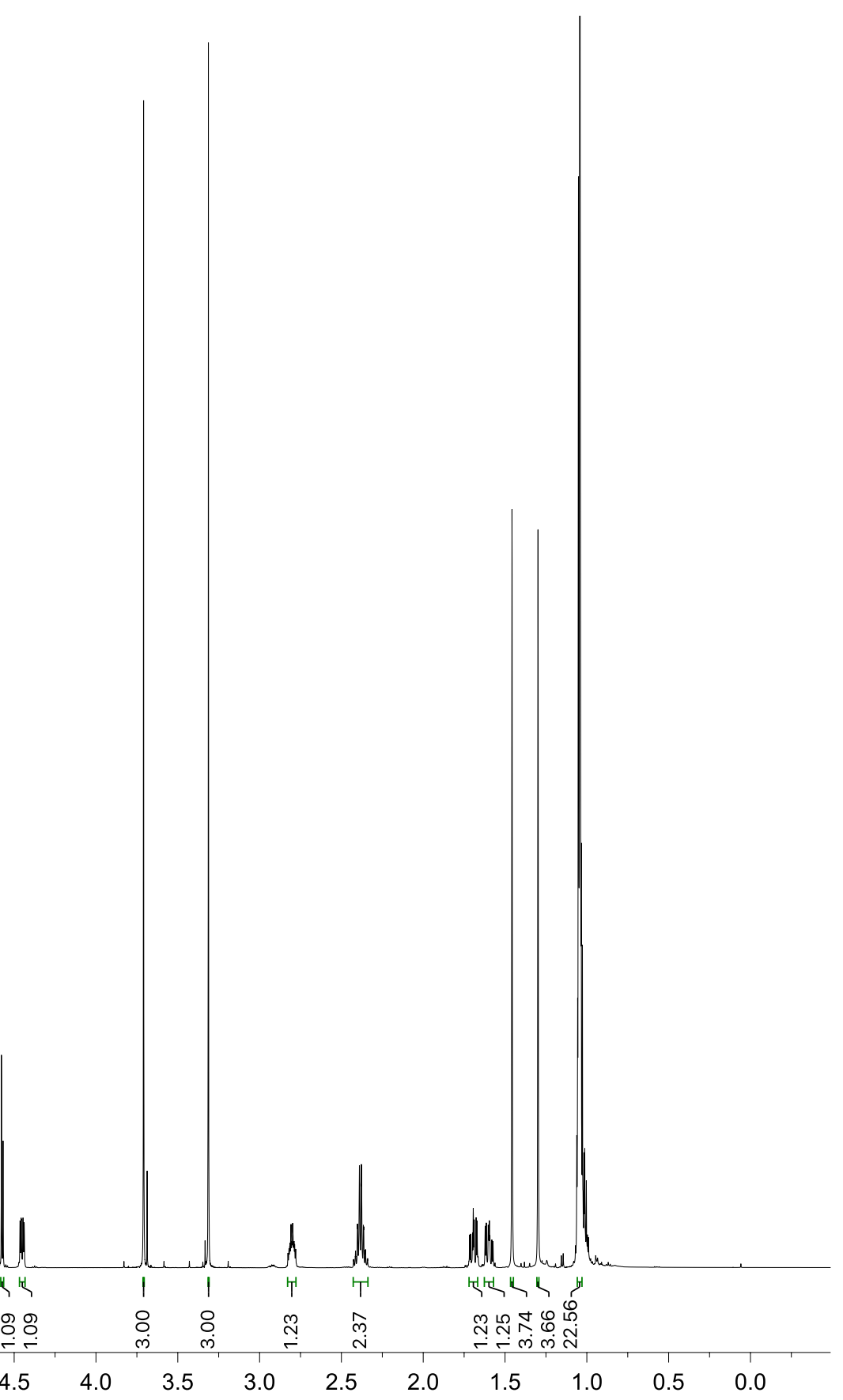



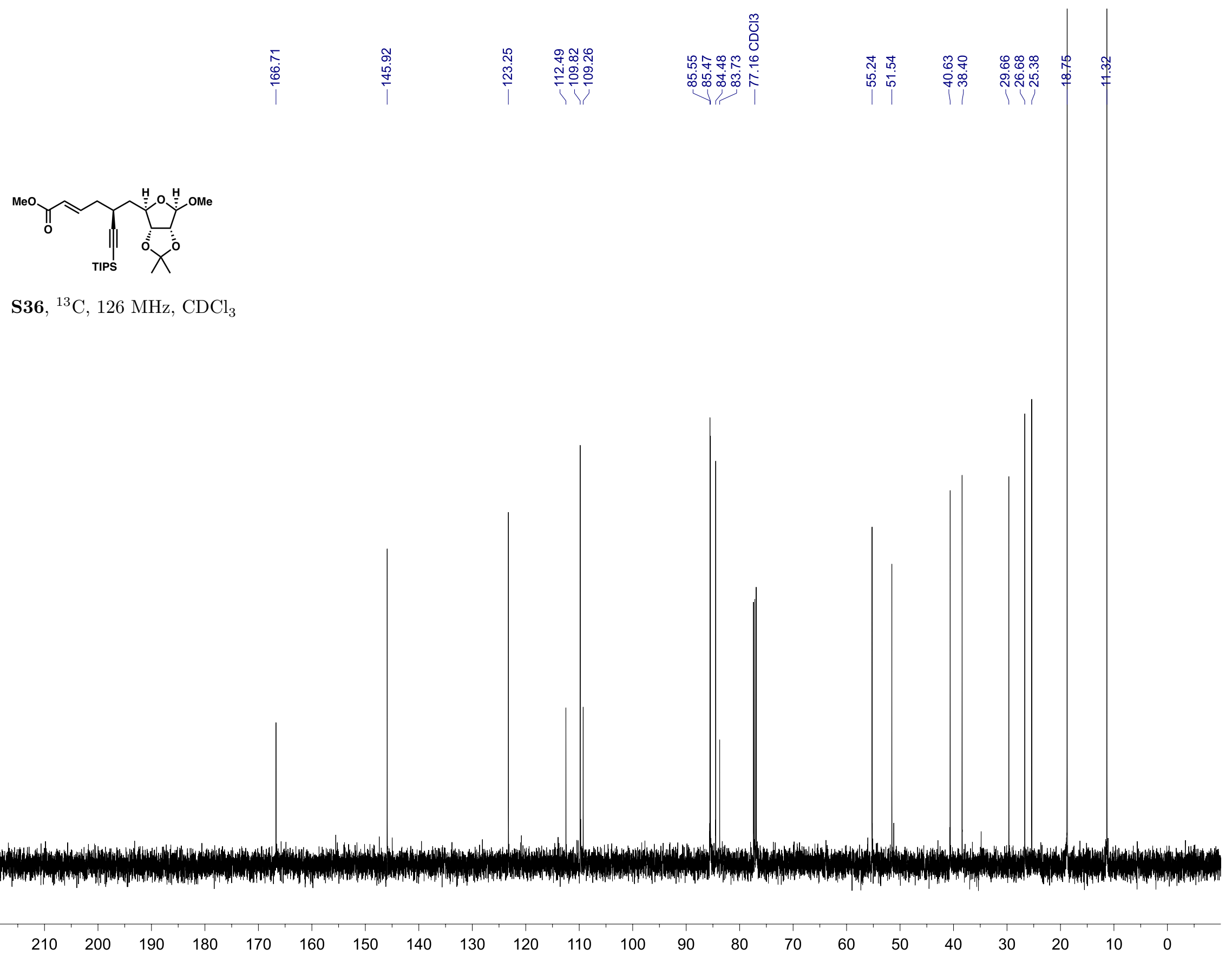


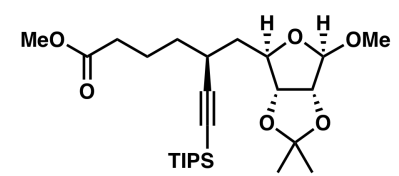

S37, ${ }^{1} \mathrm{H}, 600 \mathrm{MHz}, \mathrm{CDCl}_{3}$

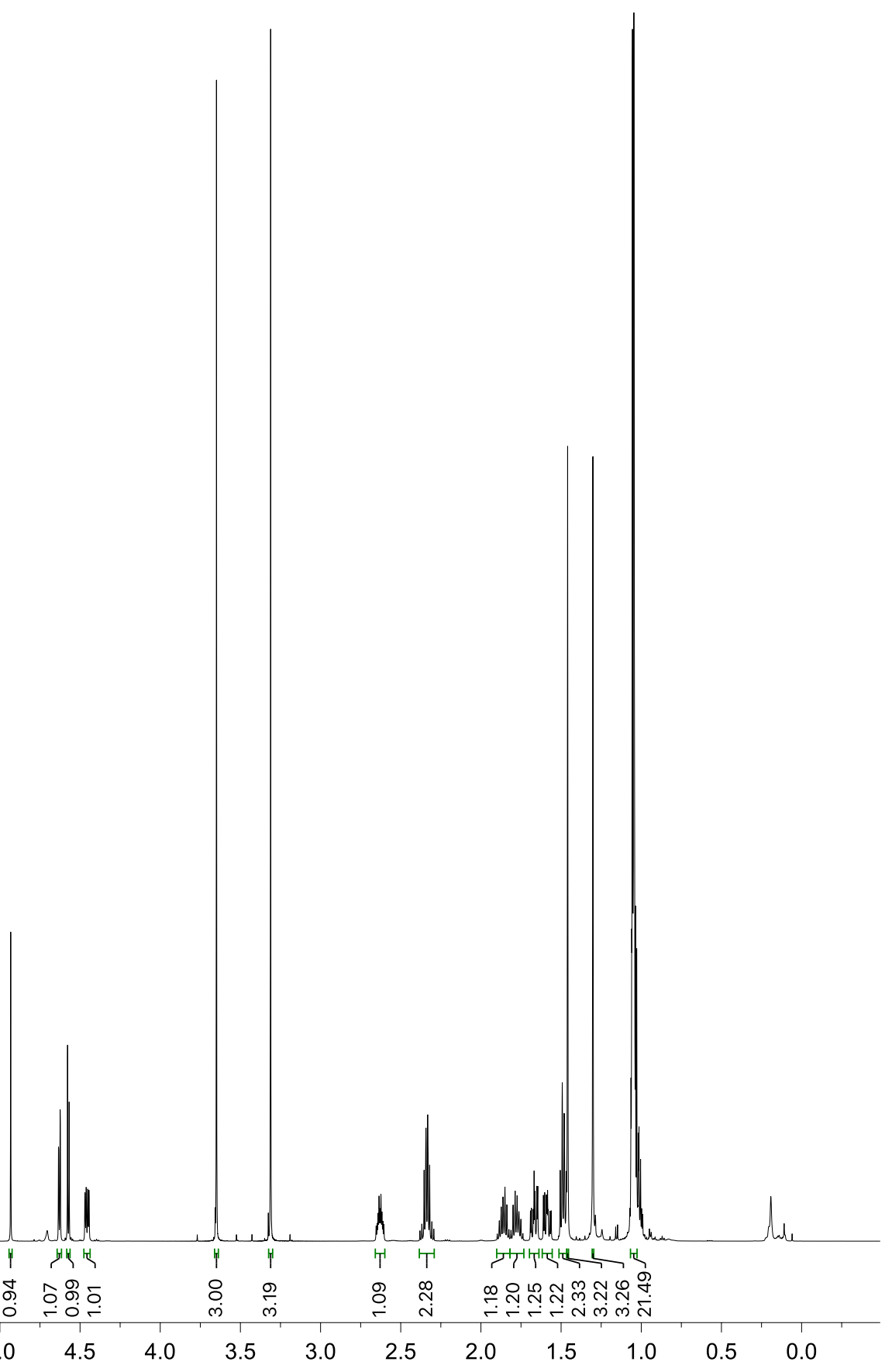




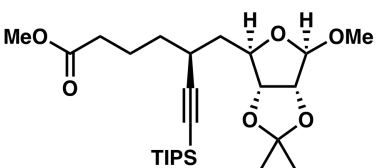

S37 $,{ }^{13} \mathrm{C}, 126 \mathrm{MHz}, \mathrm{CDCl}_{3}$ 
S38, ${ }^{1} \mathrm{H}, 600 \mathrm{MHz}, \mathrm{CDCl}_{3}$
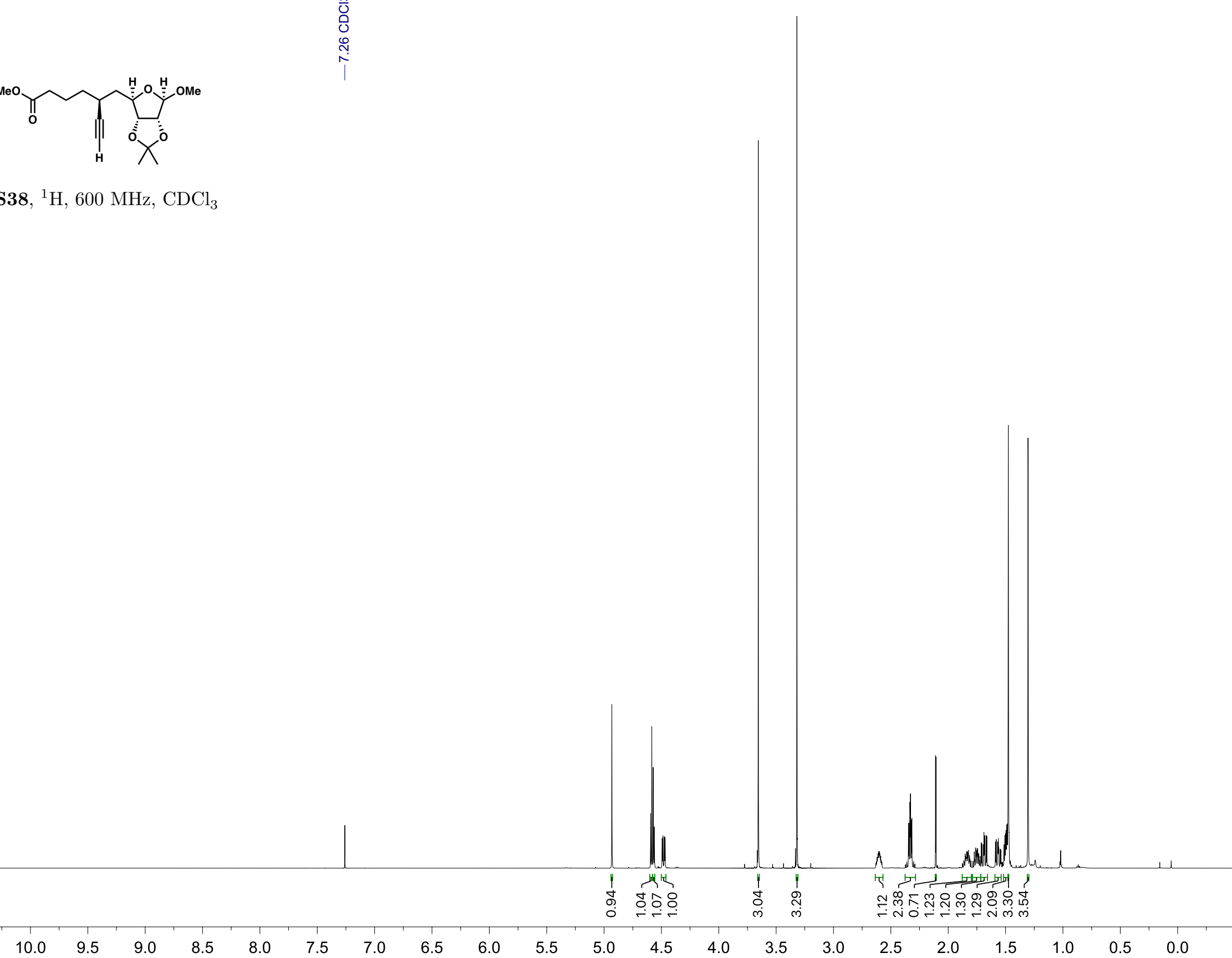


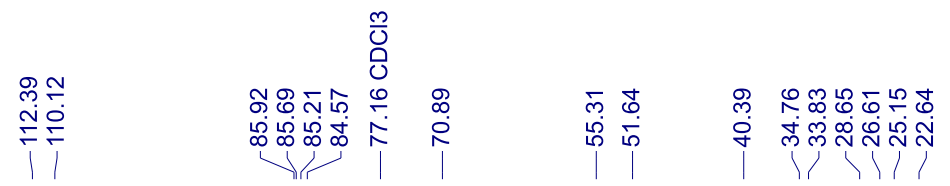

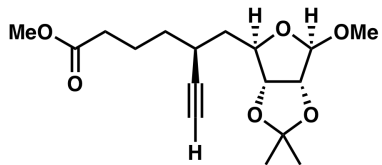

S38, ${ }^{13} \mathrm{C}, 126 \mathrm{MHz}, \mathrm{CDCl}_{3}$ 


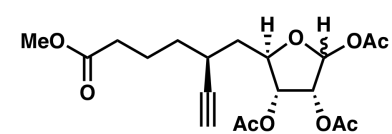

S39, ${ }^{1} \mathrm{H}, 600 \mathrm{MHz}, \mathrm{CDCl}_{3}$

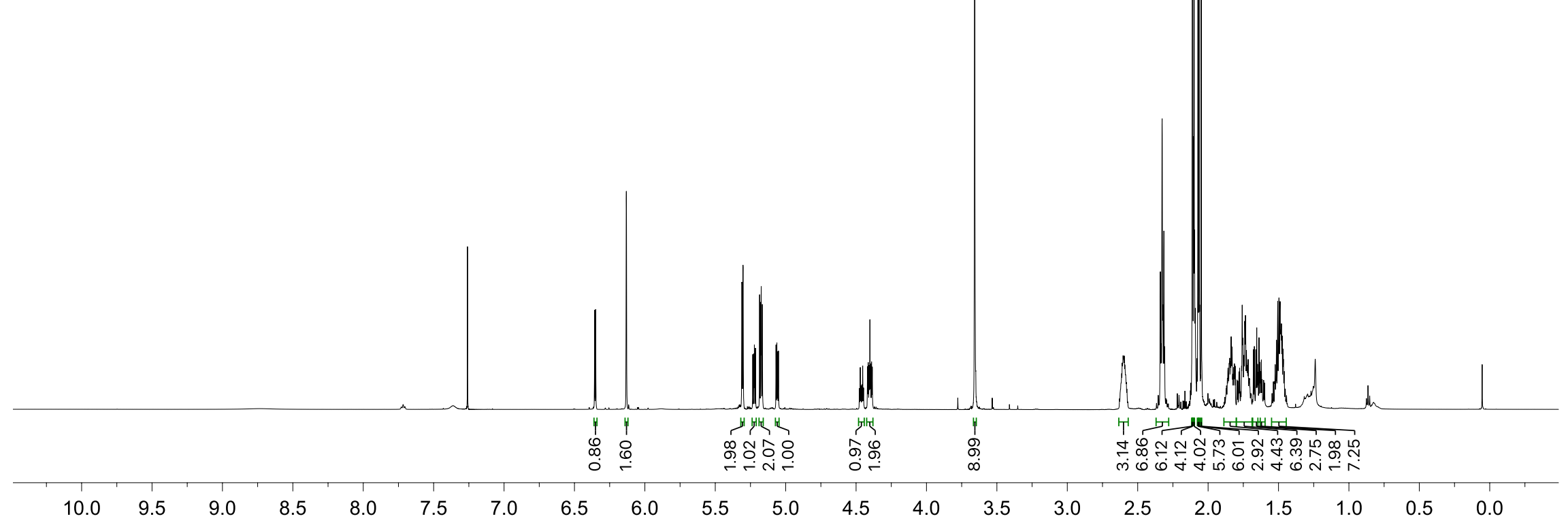




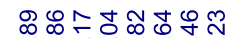

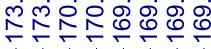

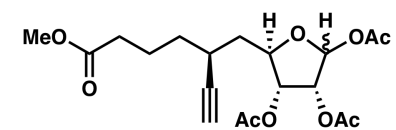

S39, ${ }^{13} \mathrm{C}, 126 \mathrm{MHz}, \mathrm{CDCl}_{3}$

\section{잉}

ธ

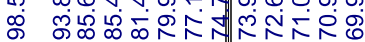

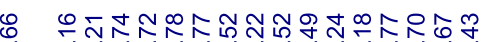

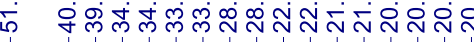




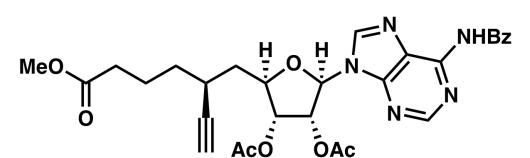

S40, ${ }^{1} \mathrm{H}, 600 \mathrm{MHz}, \mathrm{CDCl}_{3}$

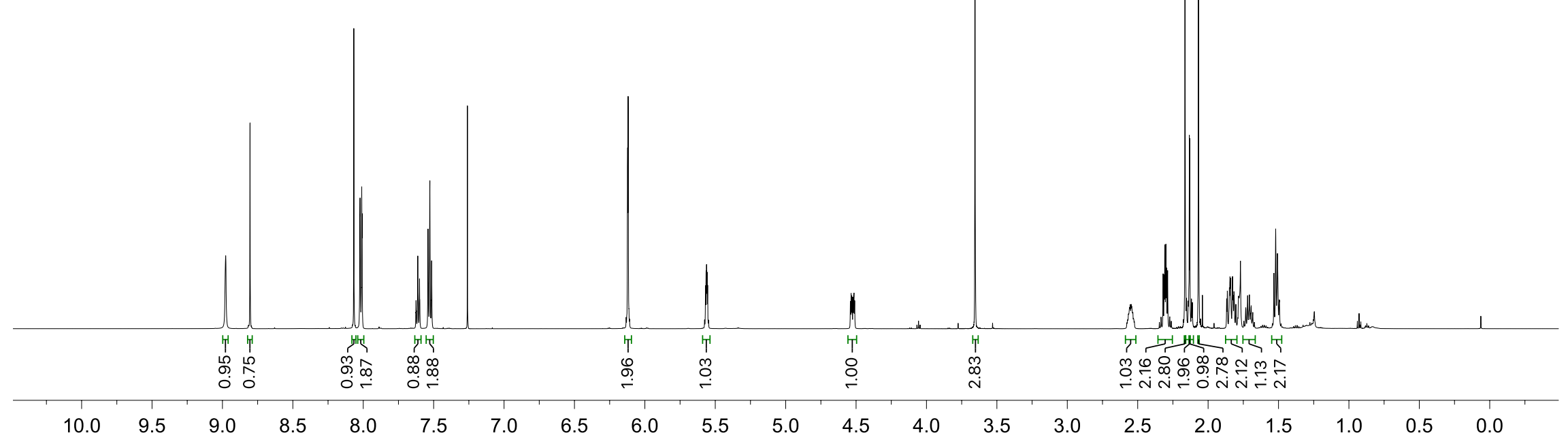




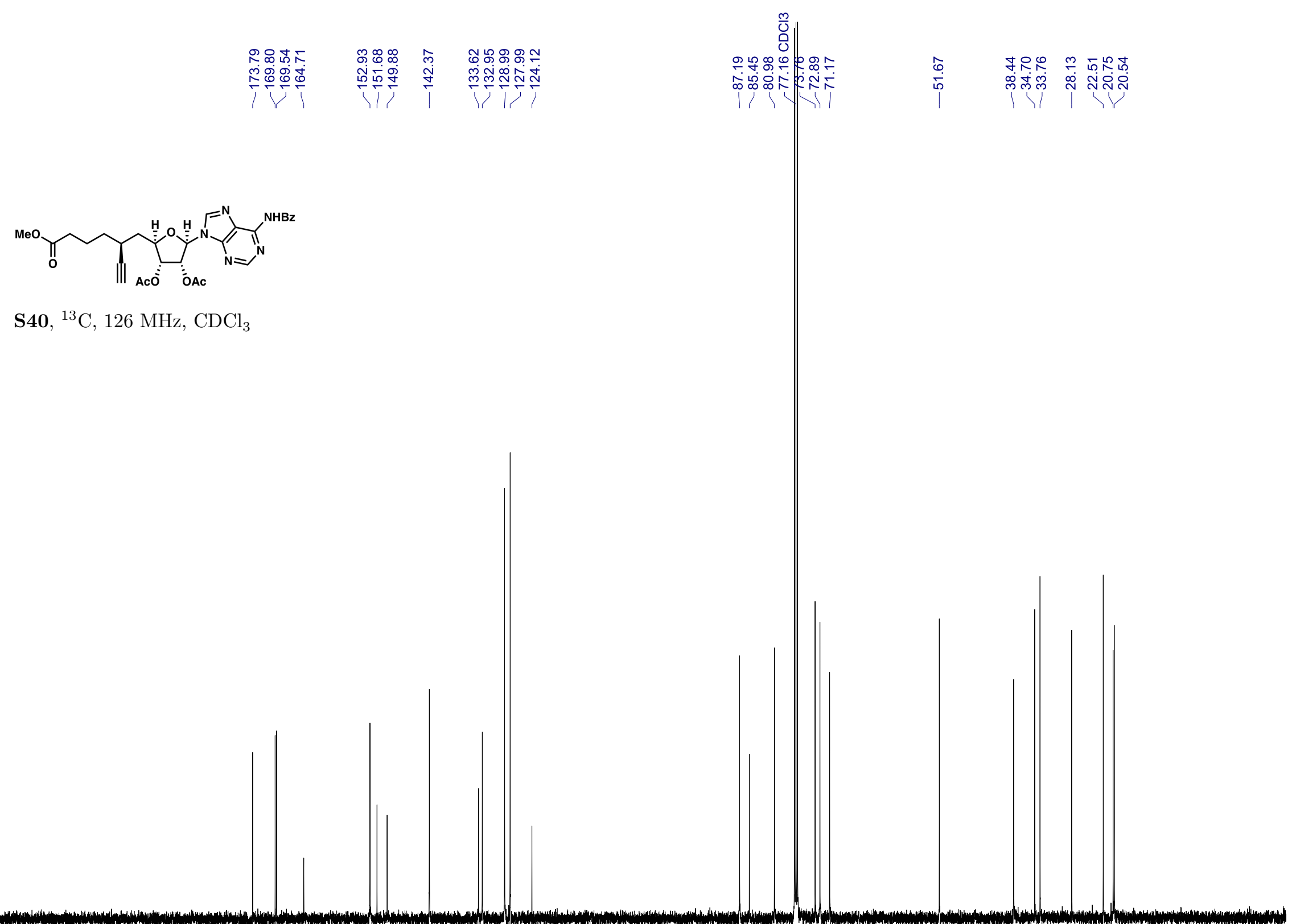




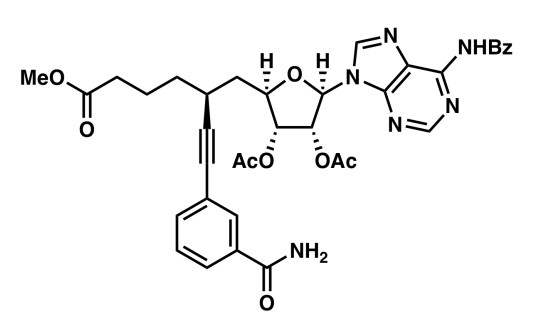

S41, ${ }^{1} \mathrm{H}, 600 \mathrm{MHz}, \mathrm{CDCl}_{3}$

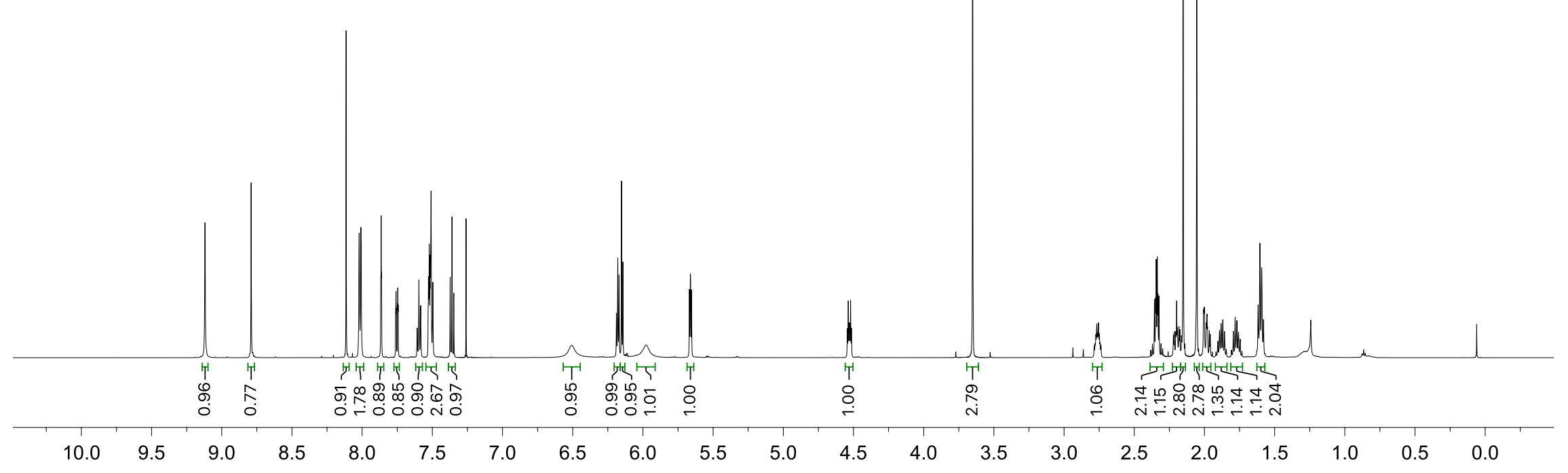




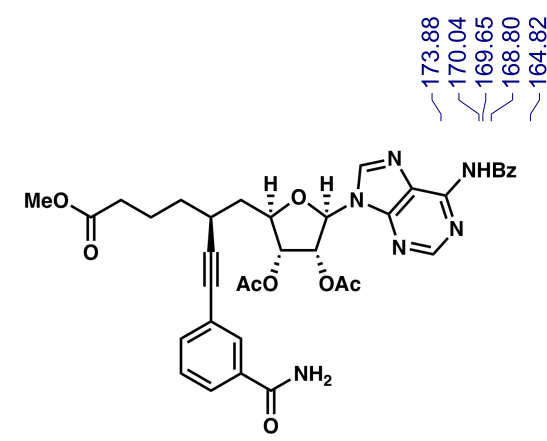

œ๐

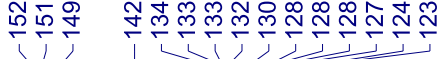

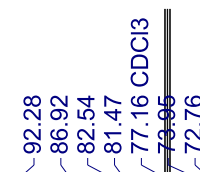

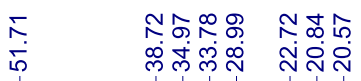

S41, ${ }^{13} \mathrm{C}, 126 \mathrm{MHz}, \mathrm{CDCl}_{3}$ 


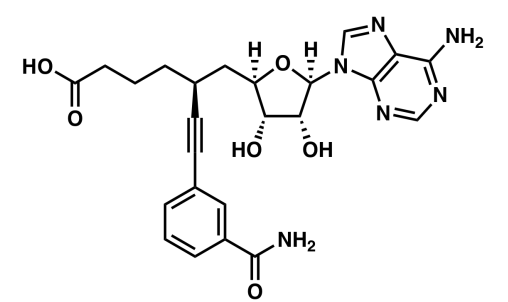

20, ${ }^{1} \mathrm{H}, 600 \mathrm{MHz}, \mathbf{C D}_{\mathbf{3}} \mathbf{C N} / \mathrm{D}_{2} \mathrm{O} / d-\mathrm{TFA}$

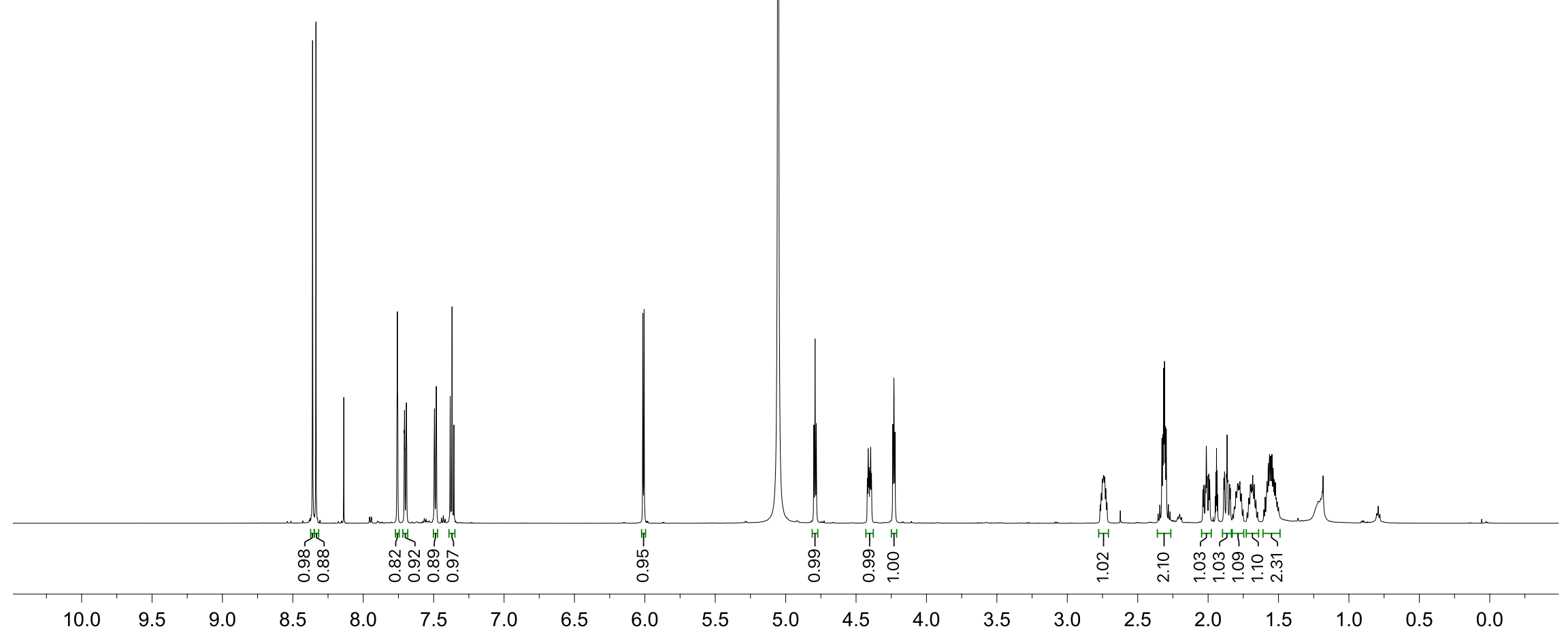




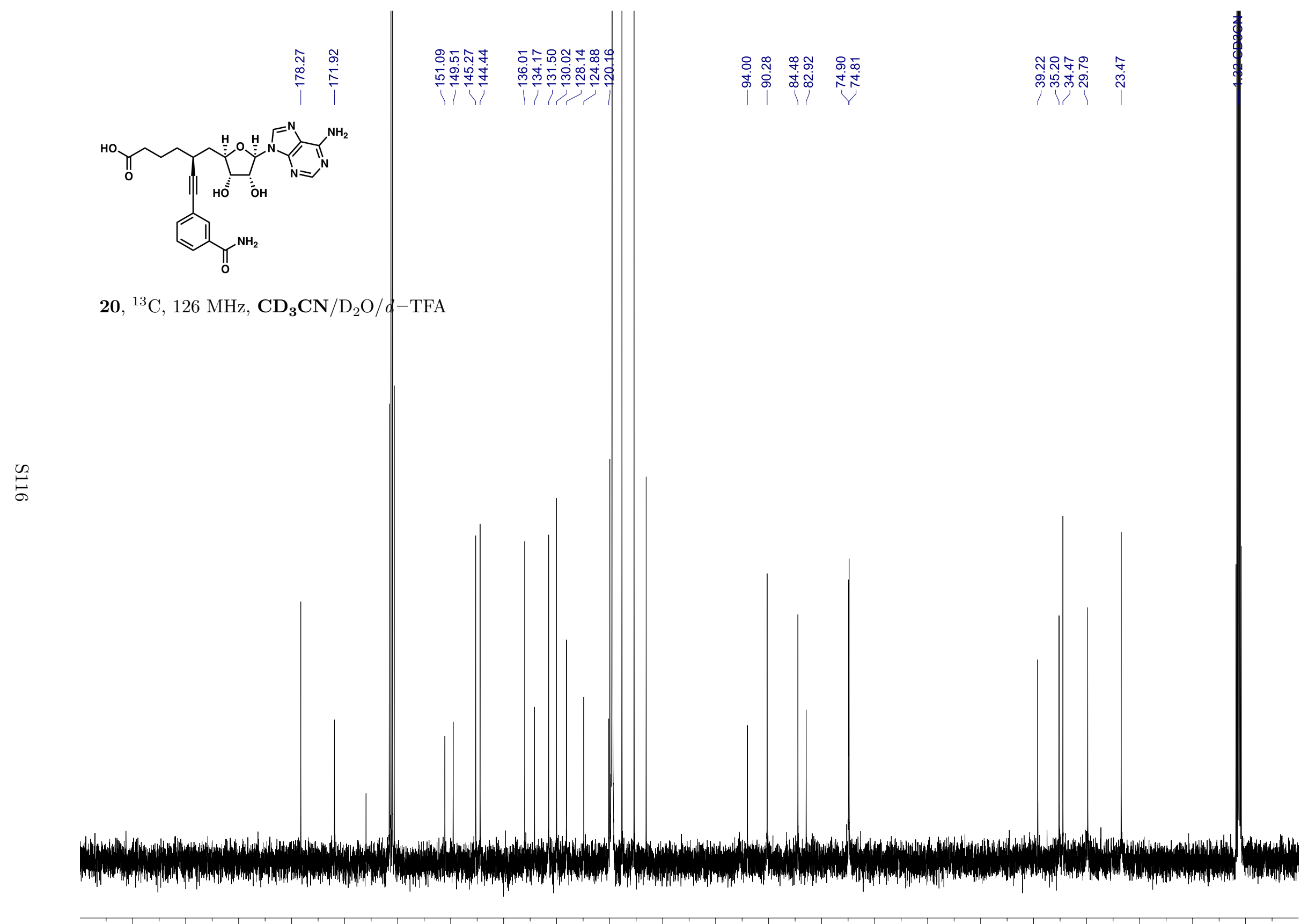

150

130

$20 \quad 110 \quad 100$

90

$80 \quad 70$

$60 \quad 50$

$40 \quad 30$

$20 \quad 10$ 0 
$\mathbf{S 4 2},{ }^{1} \mathrm{H}, 600 \mathrm{MHz}, \mathrm{CDCl}_{3}$<smiles></smiles>

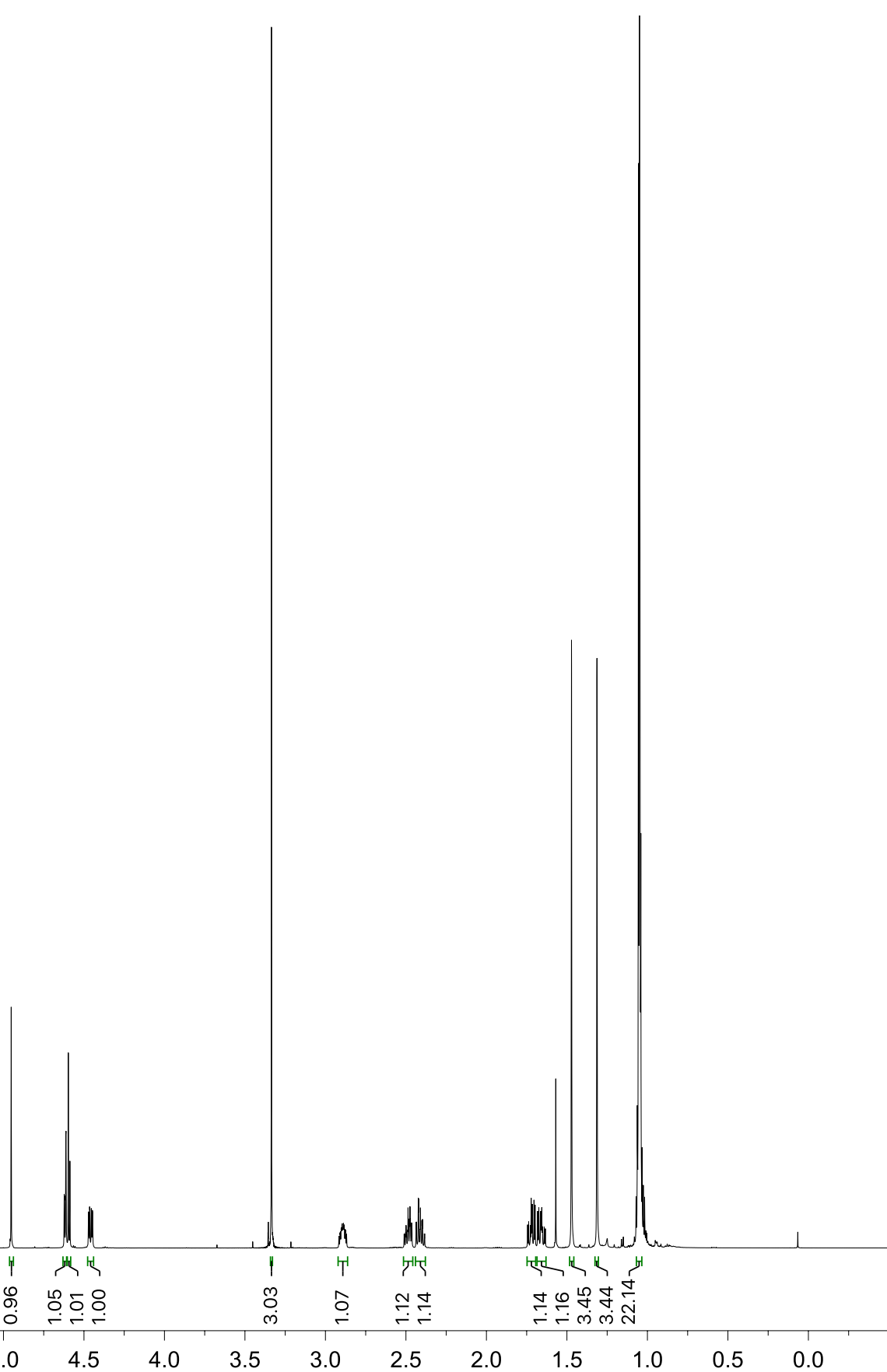




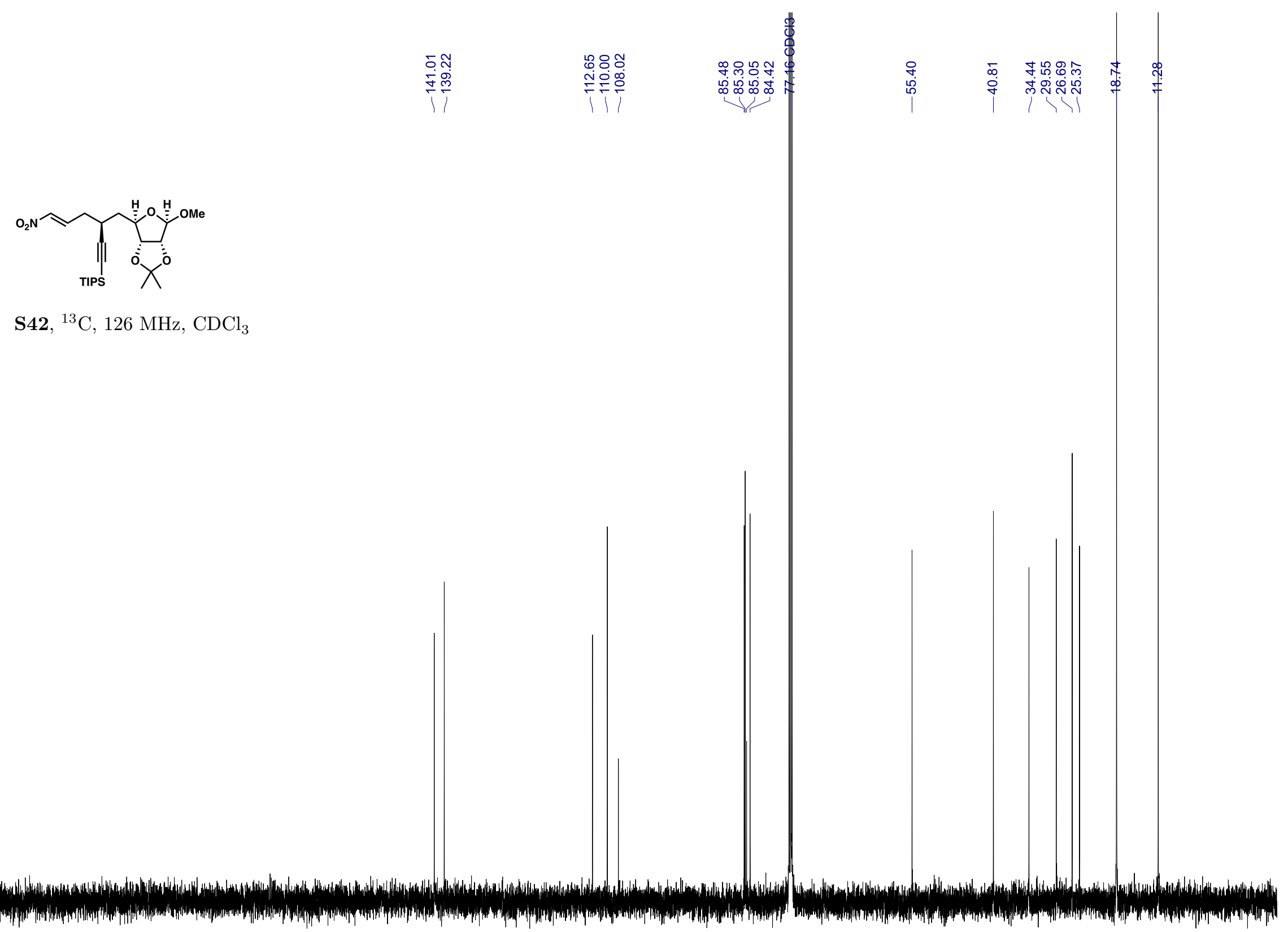

$210 \quad 200 \quad 190$

180

$170 \quad 160$

150

130

120

110100

90

80

60

50

$40 \quad 30$

20

10

0 


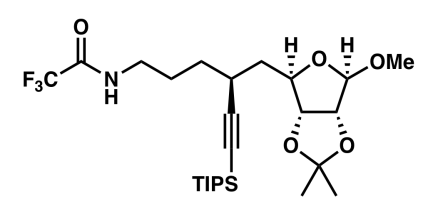

S43, ${ }^{1} \mathrm{H}, 600 \mathrm{MHz}, \mathrm{CDCl}_{3}$

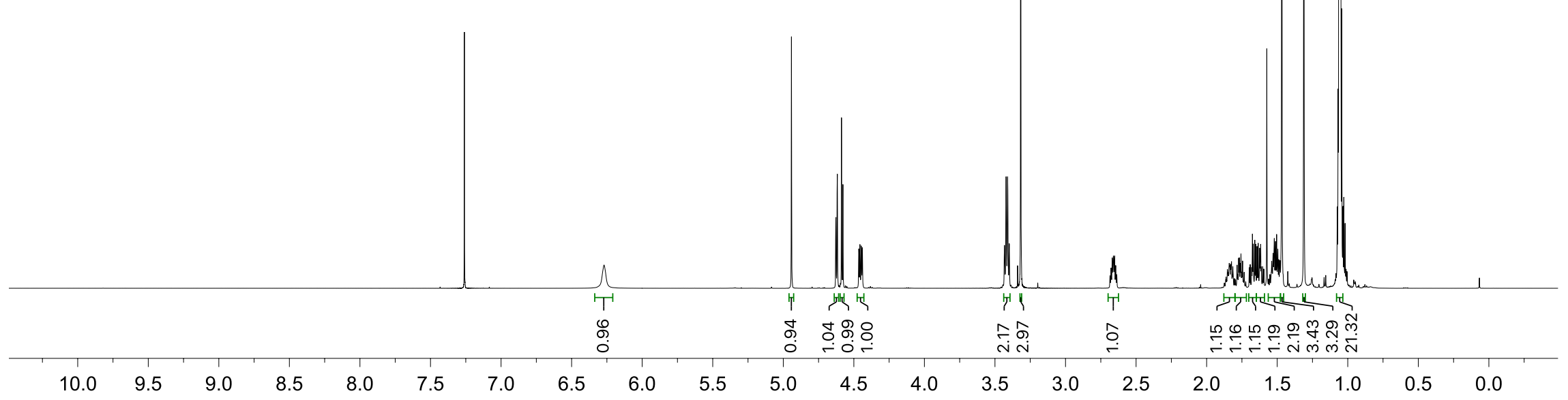




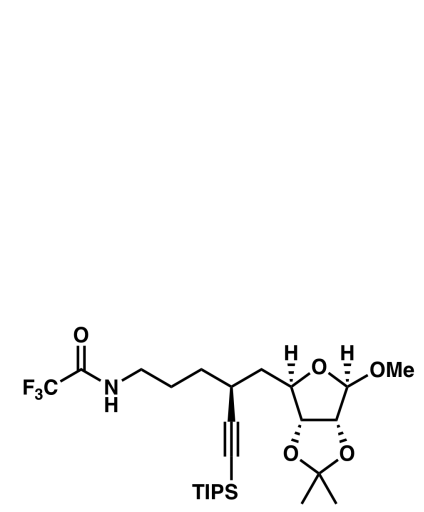

S43, ${ }^{13} \mathrm{C}, 126 \mathrm{MHz}, \mathrm{CDCl}_{3}$

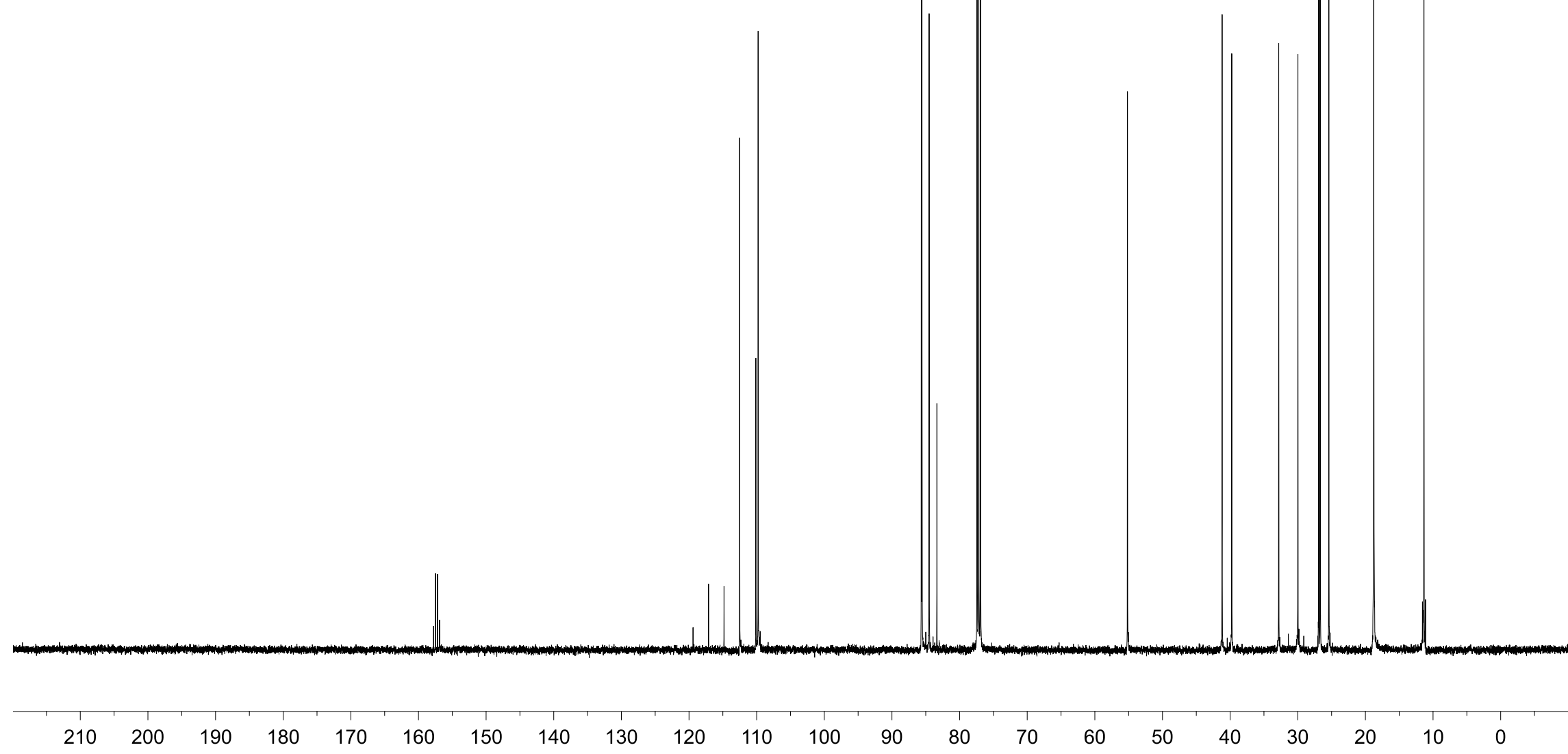




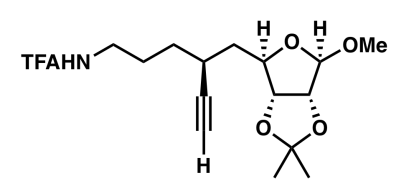

$\mathbf{S 4 4},{ }^{1} \mathrm{H}, 600 \mathrm{MHz}, \mathrm{CDCl}_{3}$

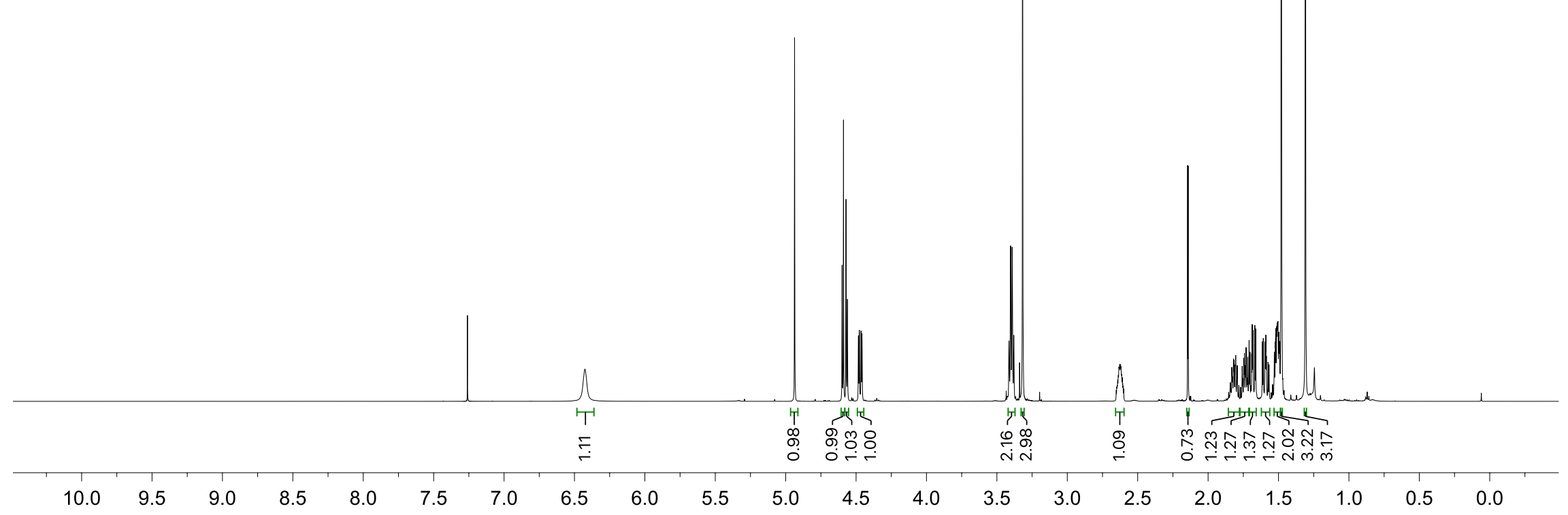


O잉

웅요

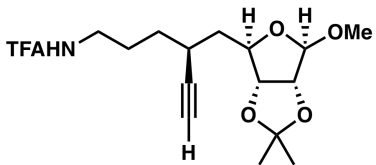

$\mathbf{S 4 4},{ }^{13} \mathrm{C}, 126 \mathrm{MHz}, \mathrm{CDCl}_{3}$

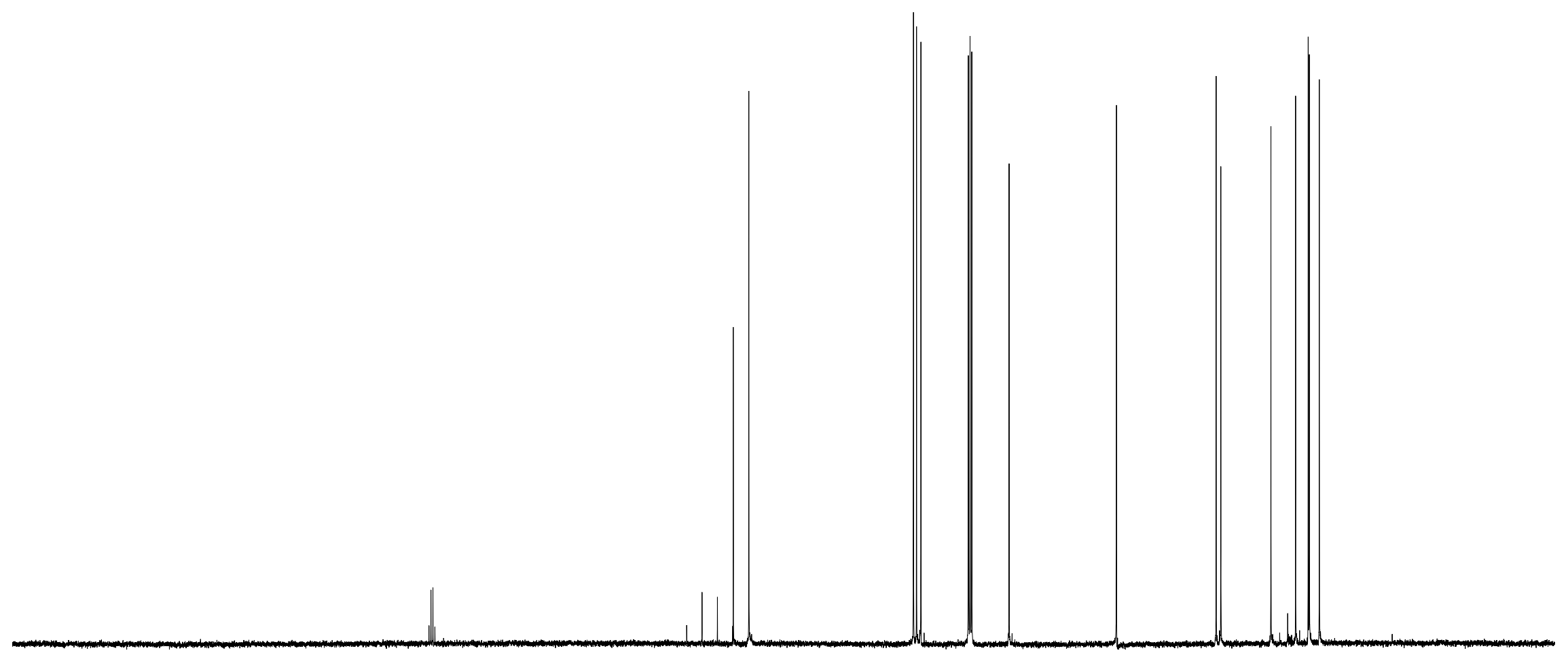




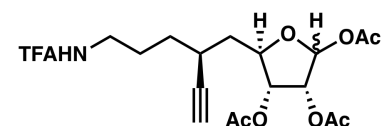

$\mathbf{S 4 5},{ }^{1} \mathrm{H}, 600 \mathrm{MHz}, \mathrm{CDCl}_{3}$

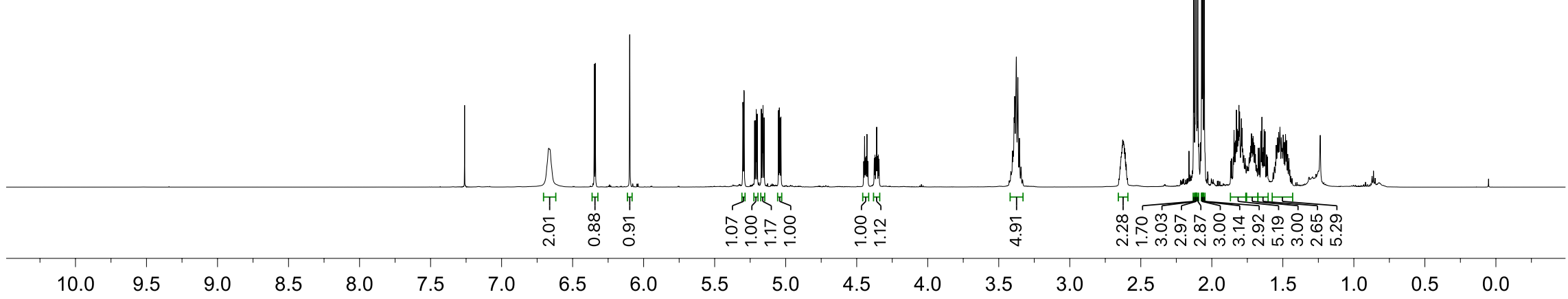




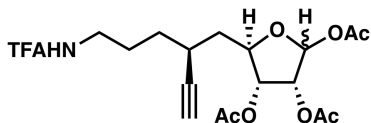

$\mathbf{S 4 5},{ }^{13} \mathrm{C}, 126 \mathrm{MHz}, \mathrm{CDCl}_{3}$

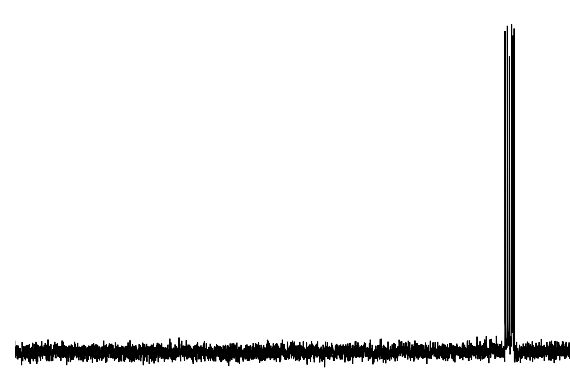




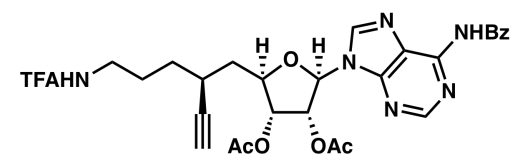

S46, ${ }^{1} \mathrm{H}, 600 \mathrm{MHz}, \mathrm{CDCl}_{3}$

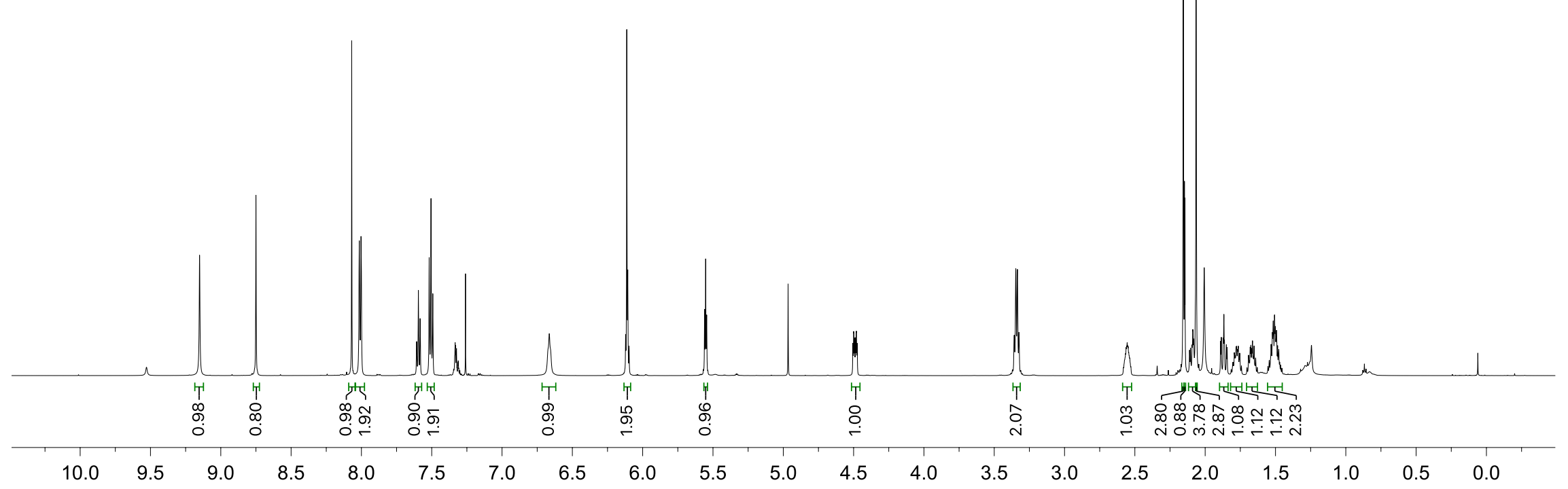




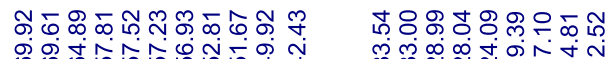

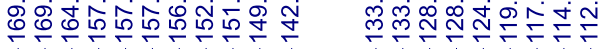

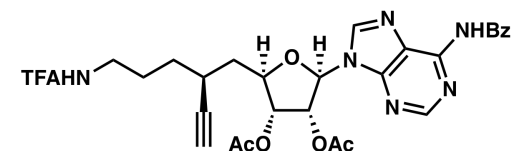

S46, ${ }^{13} \mathrm{C}, 126 \mathrm{MHz}, \mathrm{CDCl}_{3}$

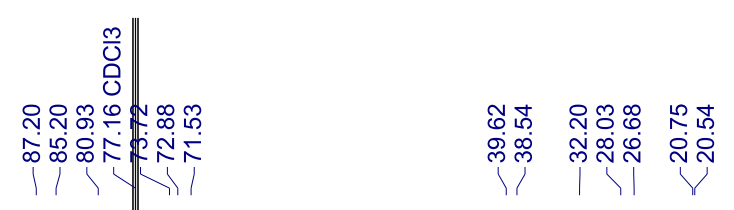




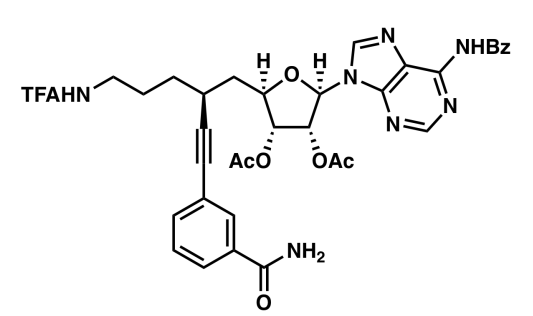

$\mathbf{S 4 7},{ }^{1} \mathrm{H}, 500 \mathrm{MHz}, \mathrm{CDCl}_{3}$

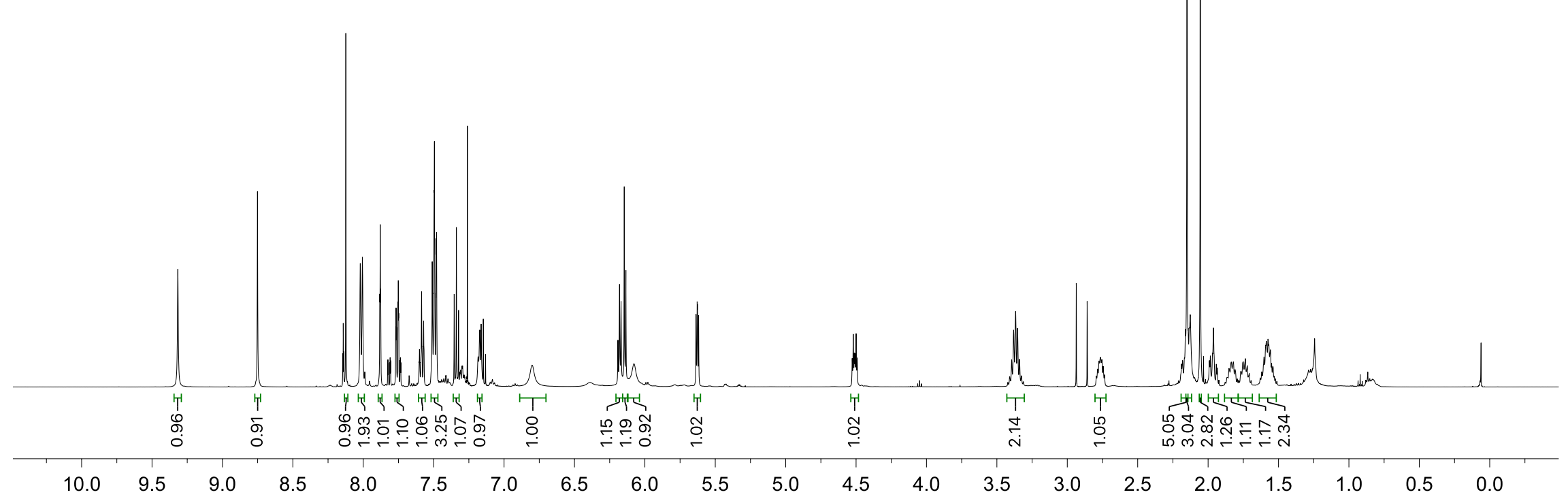




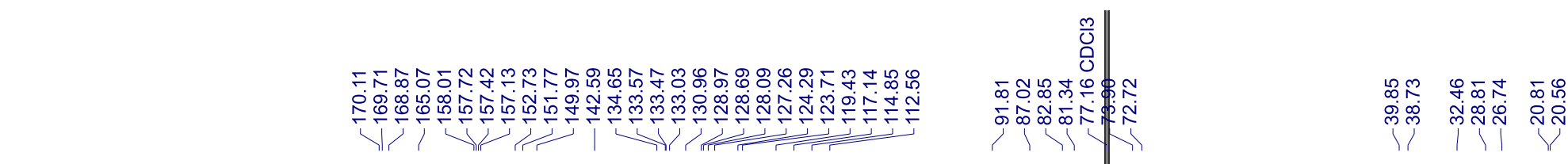

$\mathbf{S 4 7},{ }^{13} \mathrm{C}, 126 \mathrm{MHz}, \mathrm{CDCl}_{3}$

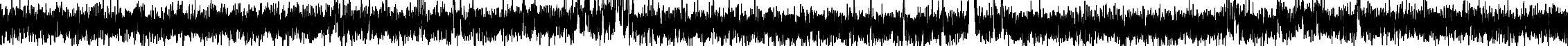




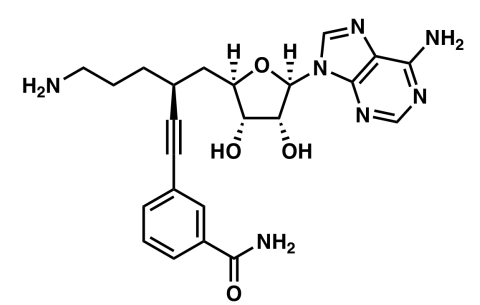

21, ${ }^{1} \mathrm{H}, 600 \mathrm{MHz}, \mathbf{C D}_{\mathbf{3}} \mathbf{C N} / \mathrm{D}_{2} \mathrm{O} / d-\mathrm{TFA}$

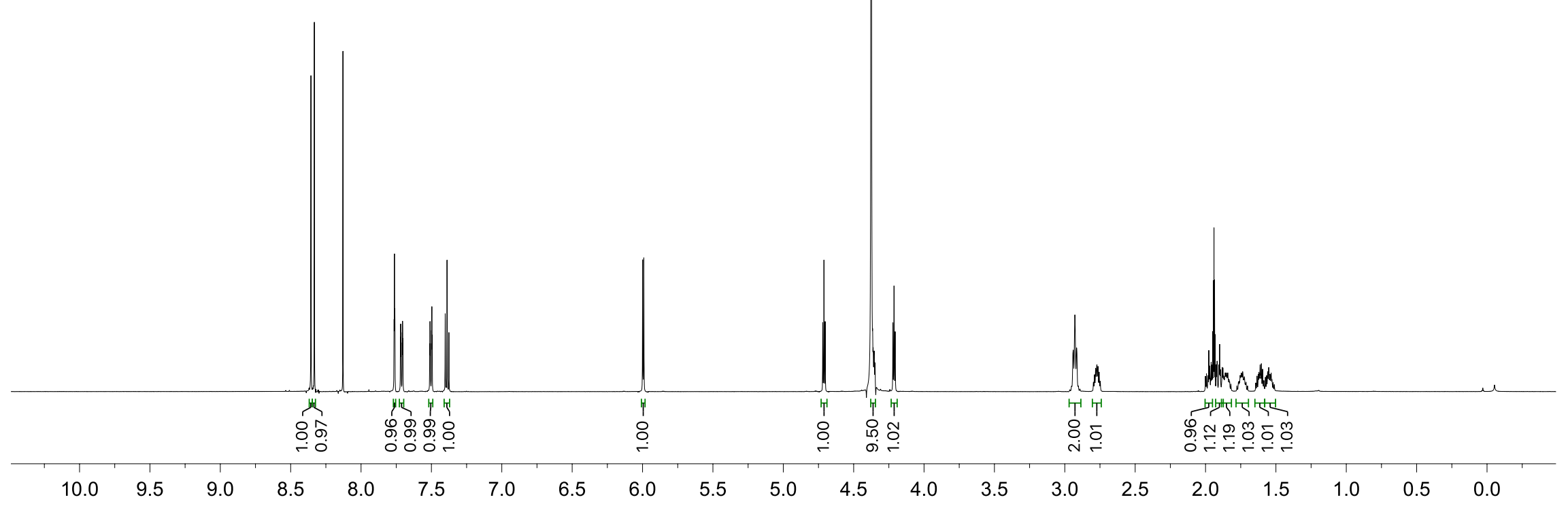




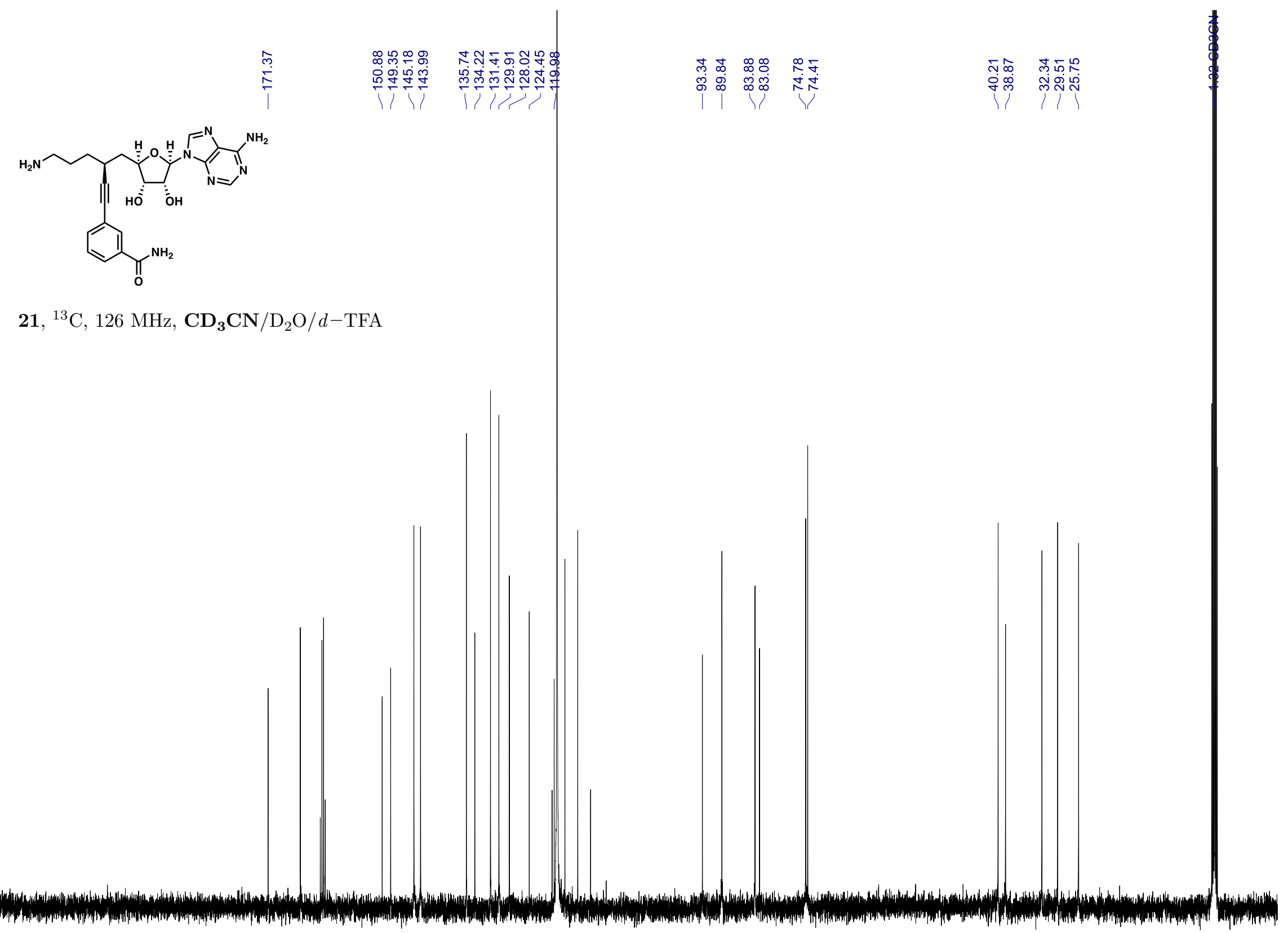

190

180

170

160

150

130

$120 \quad 110 \quad 100$

90

80

60

50

$40 \quad 30$

20 


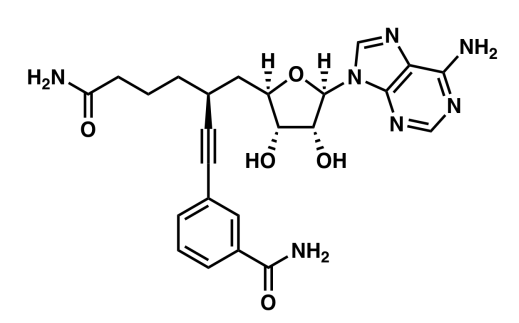

22, ${ }^{1} \mathrm{H}, 600 \mathrm{MHz}, \mathbf{C D}_{\mathbf{3}} \mathbf{C N} / \mathrm{D}_{2} \mathrm{O} / d-\mathrm{TFA}$

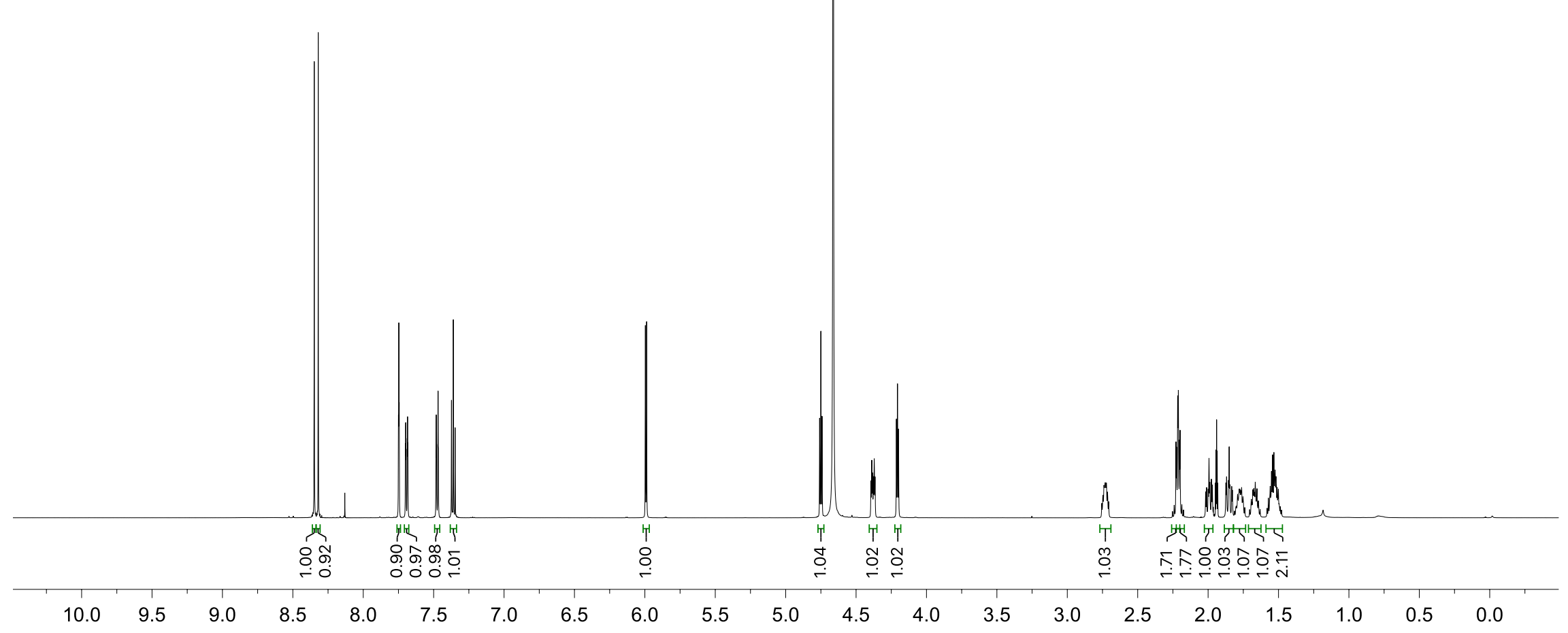




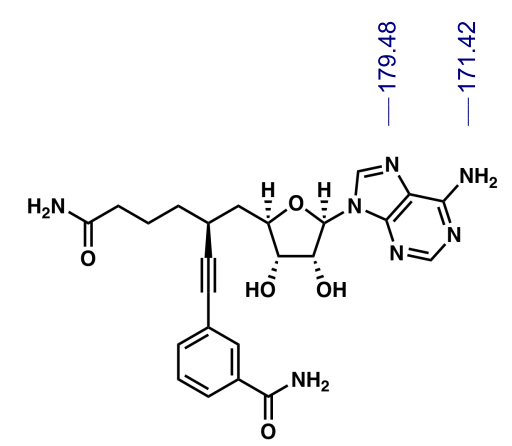

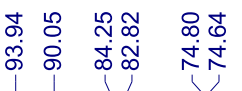

过芦造

$\mathbf{2 2},{ }^{13} \mathrm{C}, 126 \mathrm{MHz}, \mathbf{C D}_{\mathbf{3}} \mathbf{C N} / \mathrm{D}_{2} \mathrm{O} / d-\mathrm{TFA}$ 


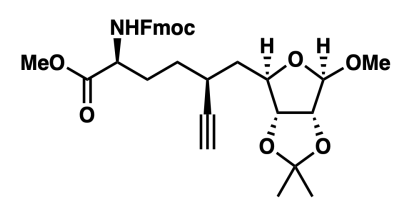

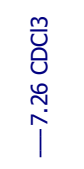

$\mathbf{S} 48,{ }^{1} \mathrm{H}, 500 \mathrm{MHz}, \mathrm{CDCl}_{3}$

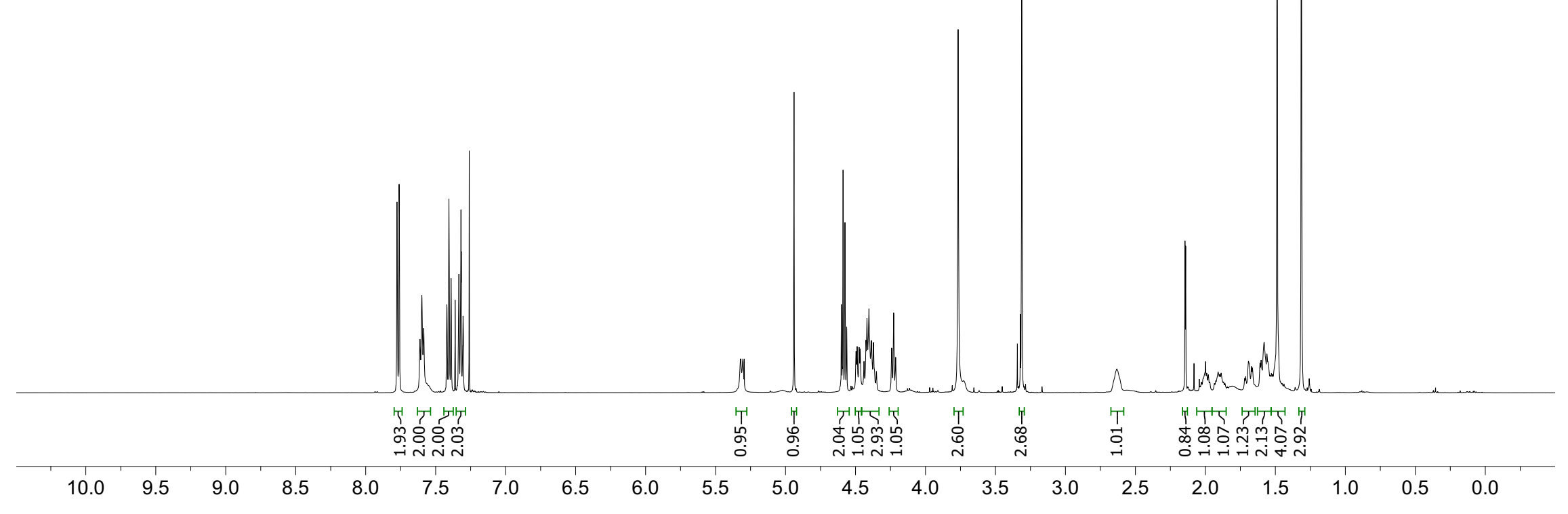




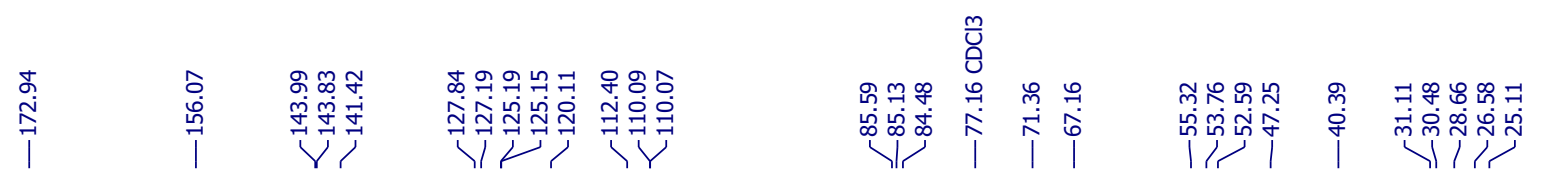

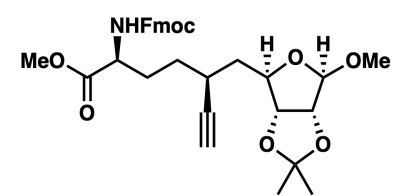

$\mathbf{S 4 8},{ }^{13} \mathrm{C}, 126 \mathrm{MHz}, \mathrm{CDCl}_{3}$ 


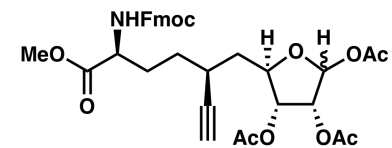

S49, ${ }^{1} \mathrm{H}, 500 \mathrm{MHz}, \mathrm{CDCl}_{3}$

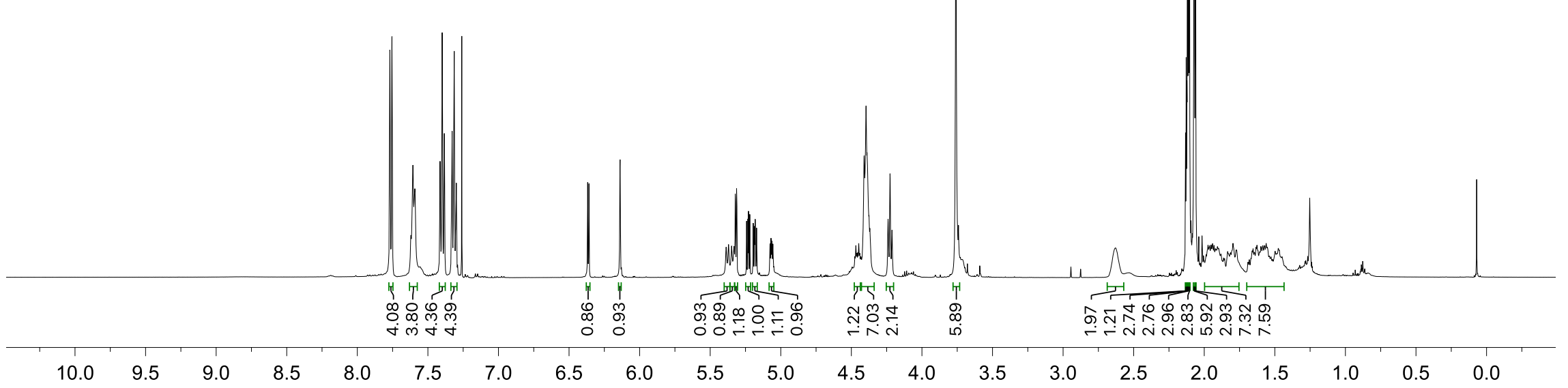




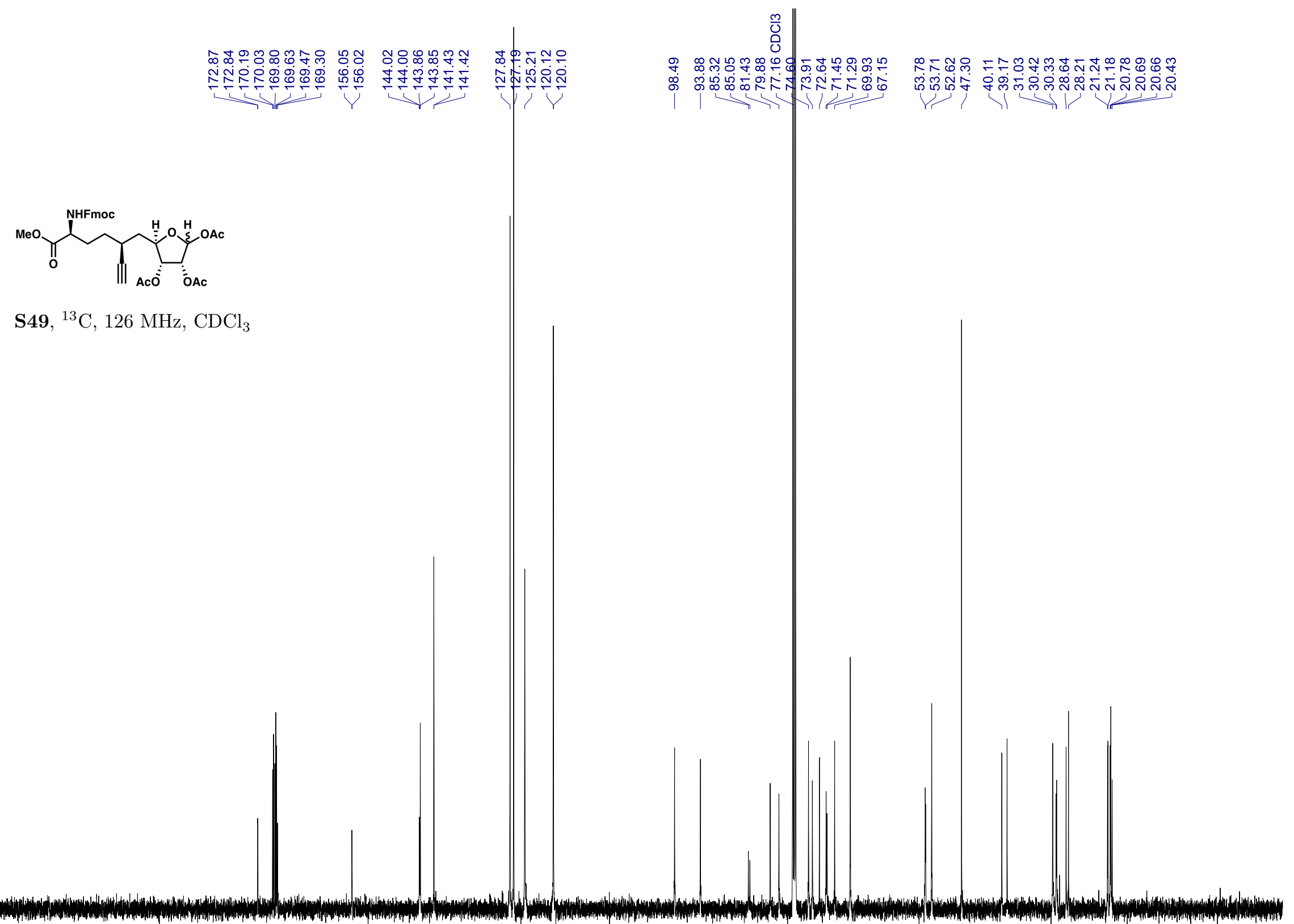

$210 \quad 200$ 


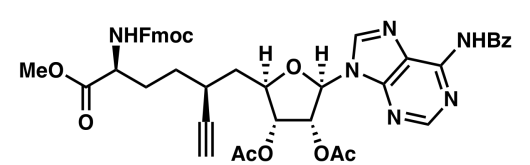

S50, ${ }^{1} \mathrm{H}, 500 \mathrm{MHz}, \mathrm{CDCl}_{3}$

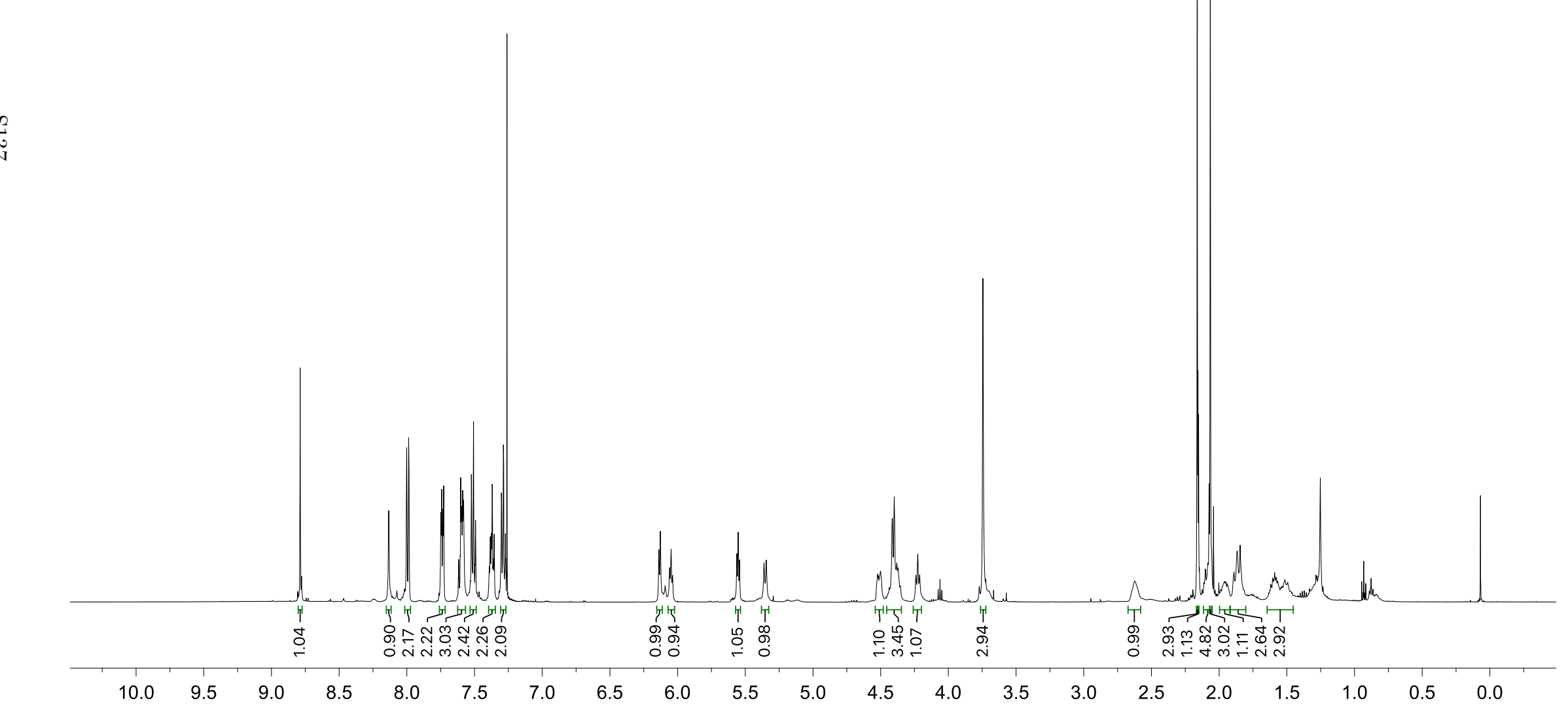




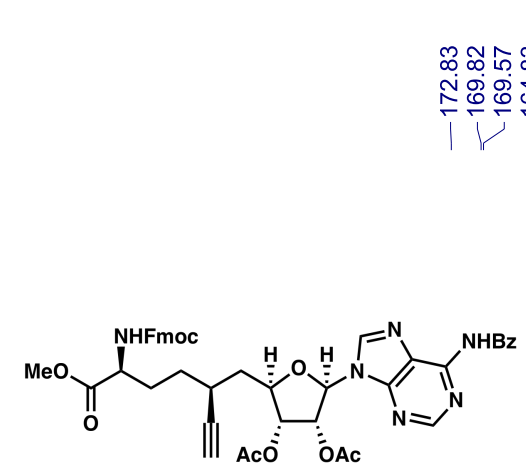

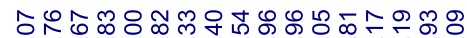

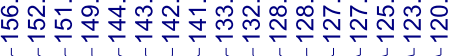

S50, ${ }^{13} \mathrm{C}, 126 \mathrm{MHz}, \mathrm{CDCl}_{3}$

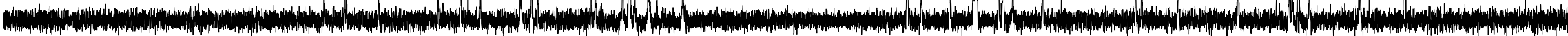




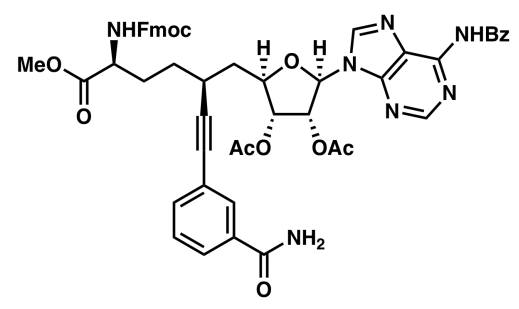

S51, ${ }^{1} \mathrm{H}, 500 \mathrm{MHz}, \mathrm{CDCl}_{3}$

$\stackrel{\pi}{\mathscr{2}}$

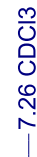
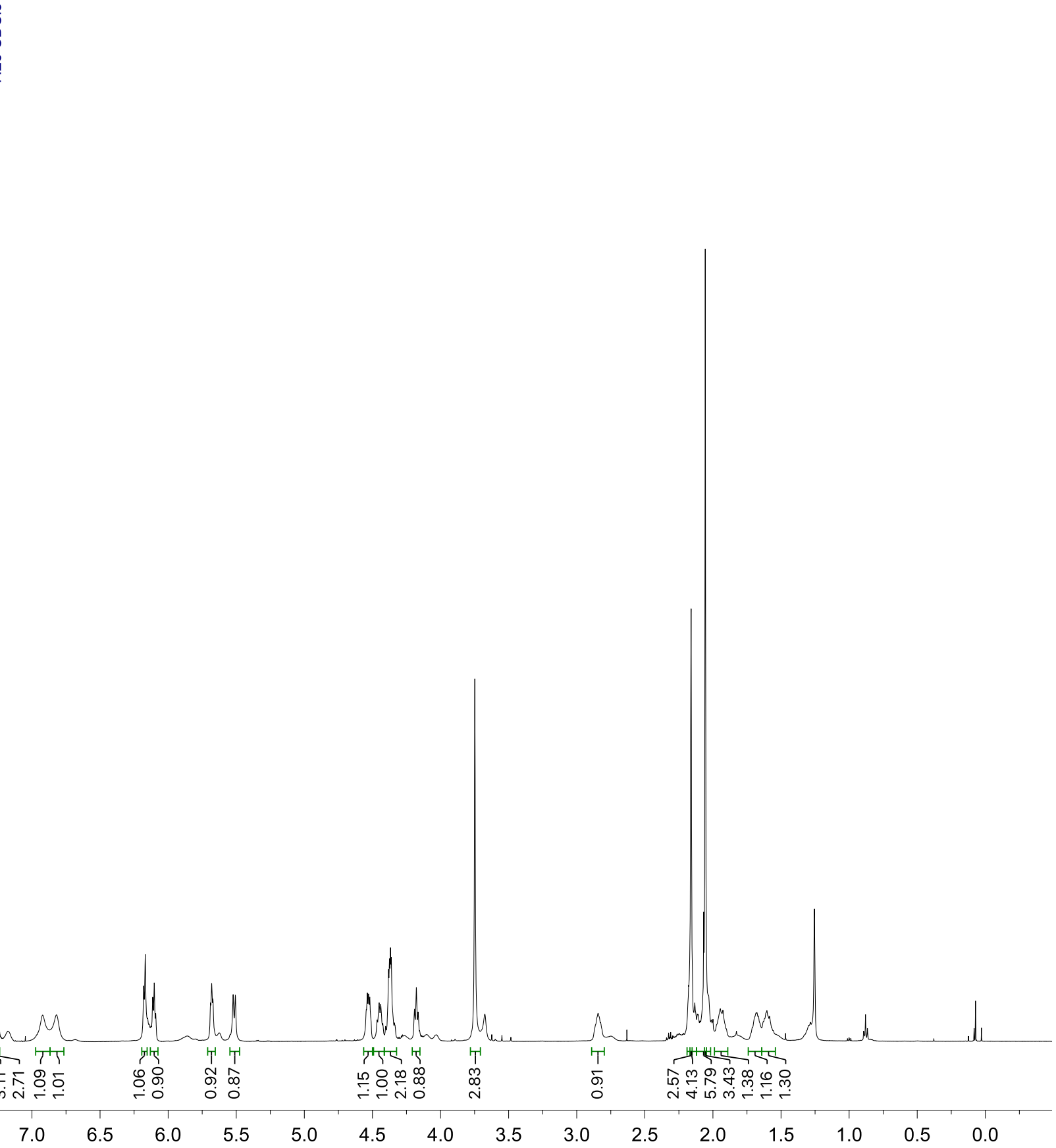

바넝

T)

$3.5 \quad 3.0 \quad 2.5$
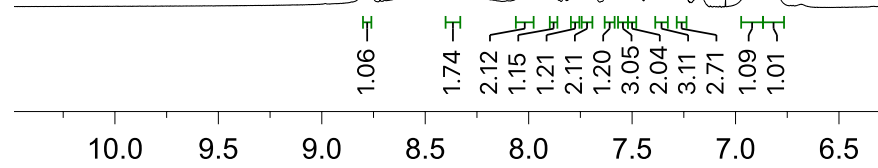

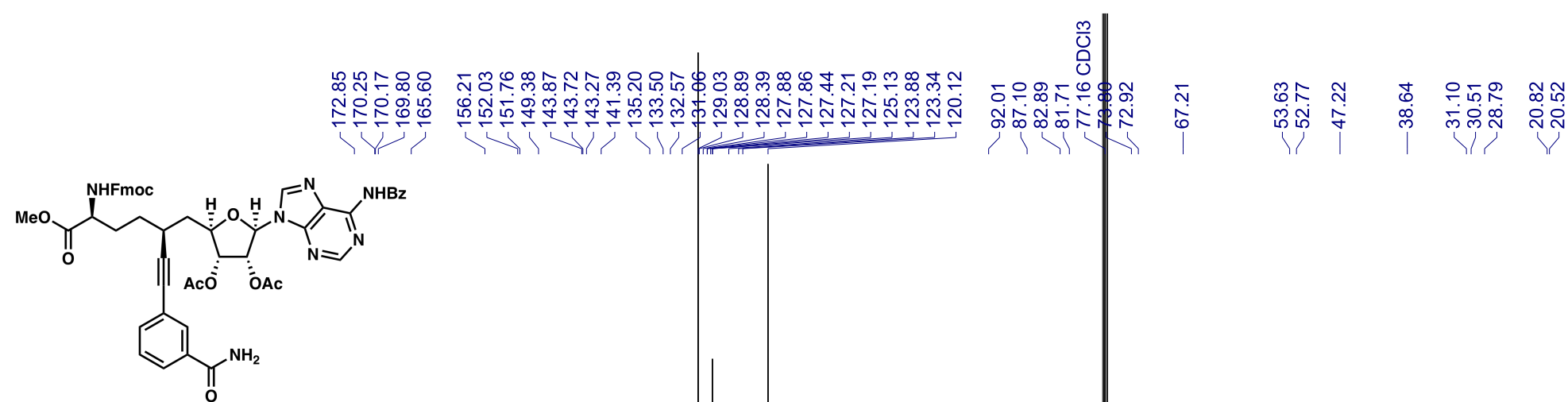

S51, ${ }^{13} \mathrm{C}, 126 \mathrm{MHz}, \mathrm{CDCl}_{3}$ 


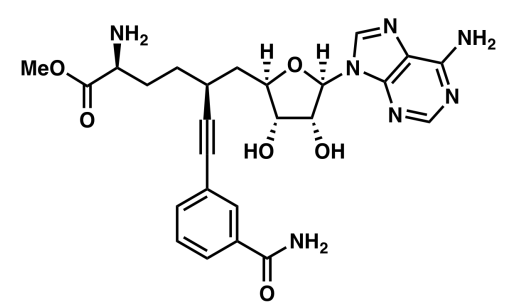

23, ${ }^{1} \mathrm{H}, 600 \mathrm{MHz}, \mathbf{C D}_{\mathbf{3}} \mathbf{C N} / \mathrm{D}_{2} \mathrm{O}$

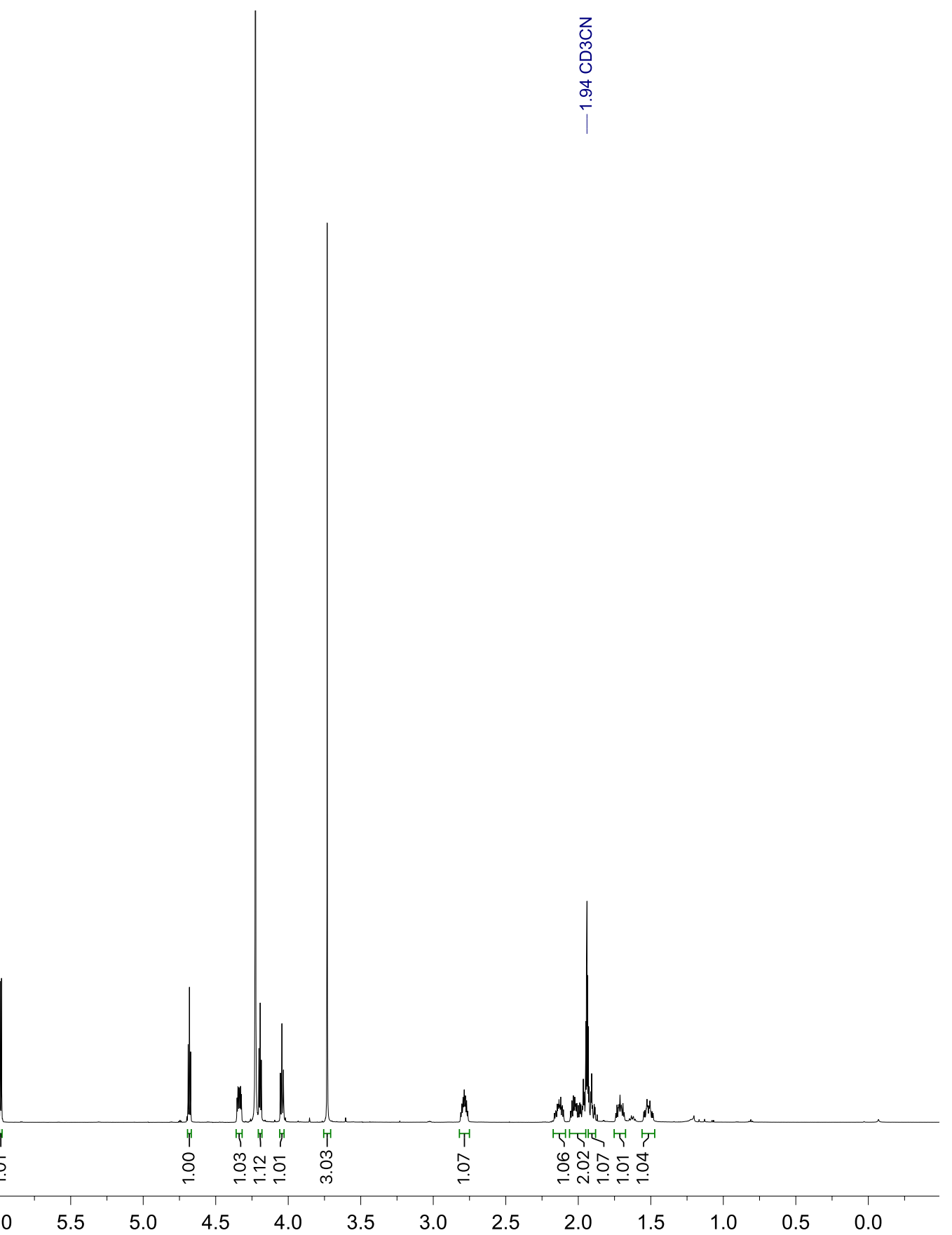




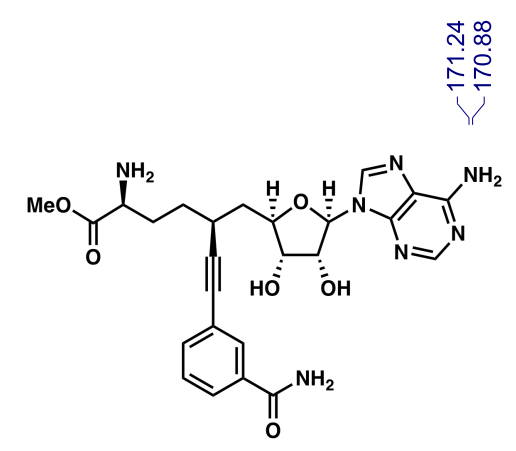

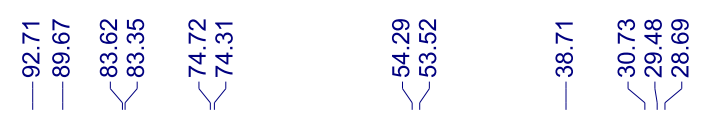

23, ${ }^{13} \mathrm{C}, 126 \mathrm{MHz}, \mathbf{C D}_{\mathbf{3}} \mathbf{C N} / \mathrm{D}_{2} \mathrm{O}$ 


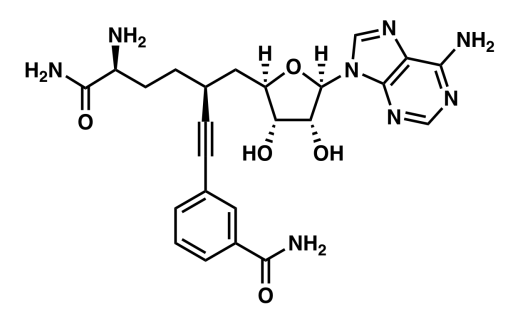

24, ${ }^{1} \mathrm{H}, 600 \mathrm{MHz}, \mathbf{C D}_{\mathbf{3}} \mathbf{C N} / \mathrm{D}_{2} \mathrm{O} / d-\mathrm{TFA}$

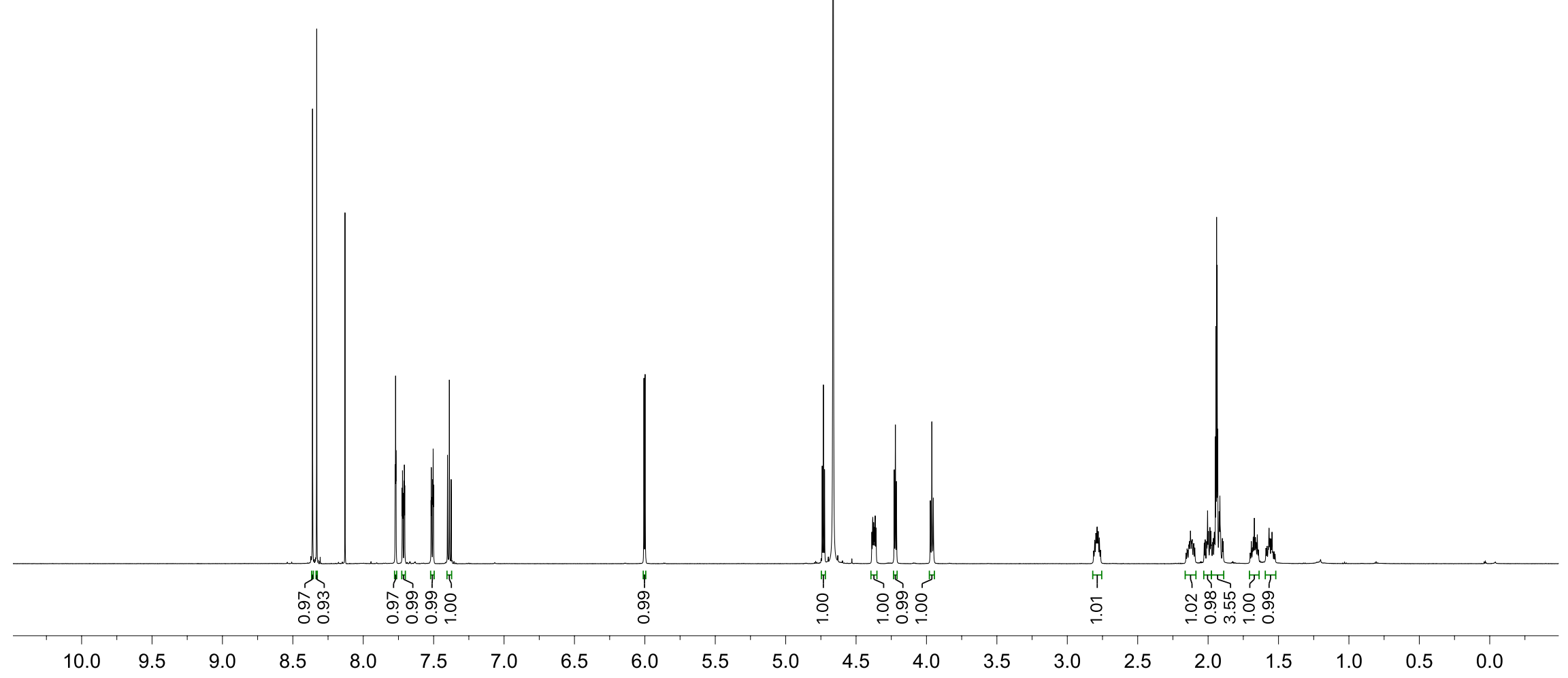




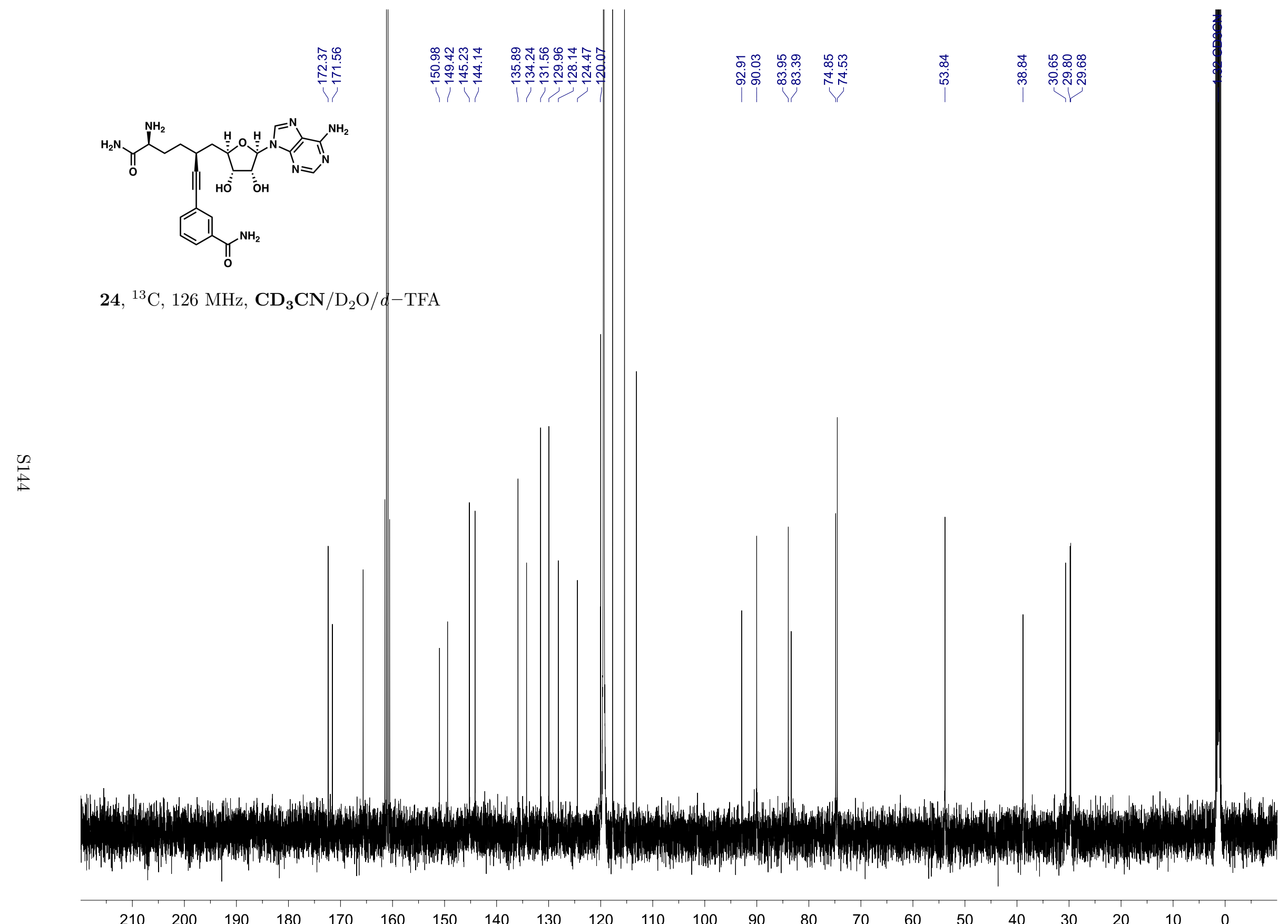

$\begin{array}{llllll}210 & 200 & 190 & 180 & 170 & 160\end{array}$

$\begin{array}{llll}150 & 140 & 130 & 120\end{array}$

60

$40 \quad 30$

$20 \quad 10$

0 


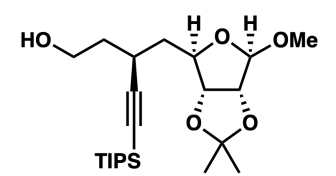

S52, ${ }^{1} \mathrm{H}, 600 \mathrm{MHz}, \mathrm{CDCl}_{3}$

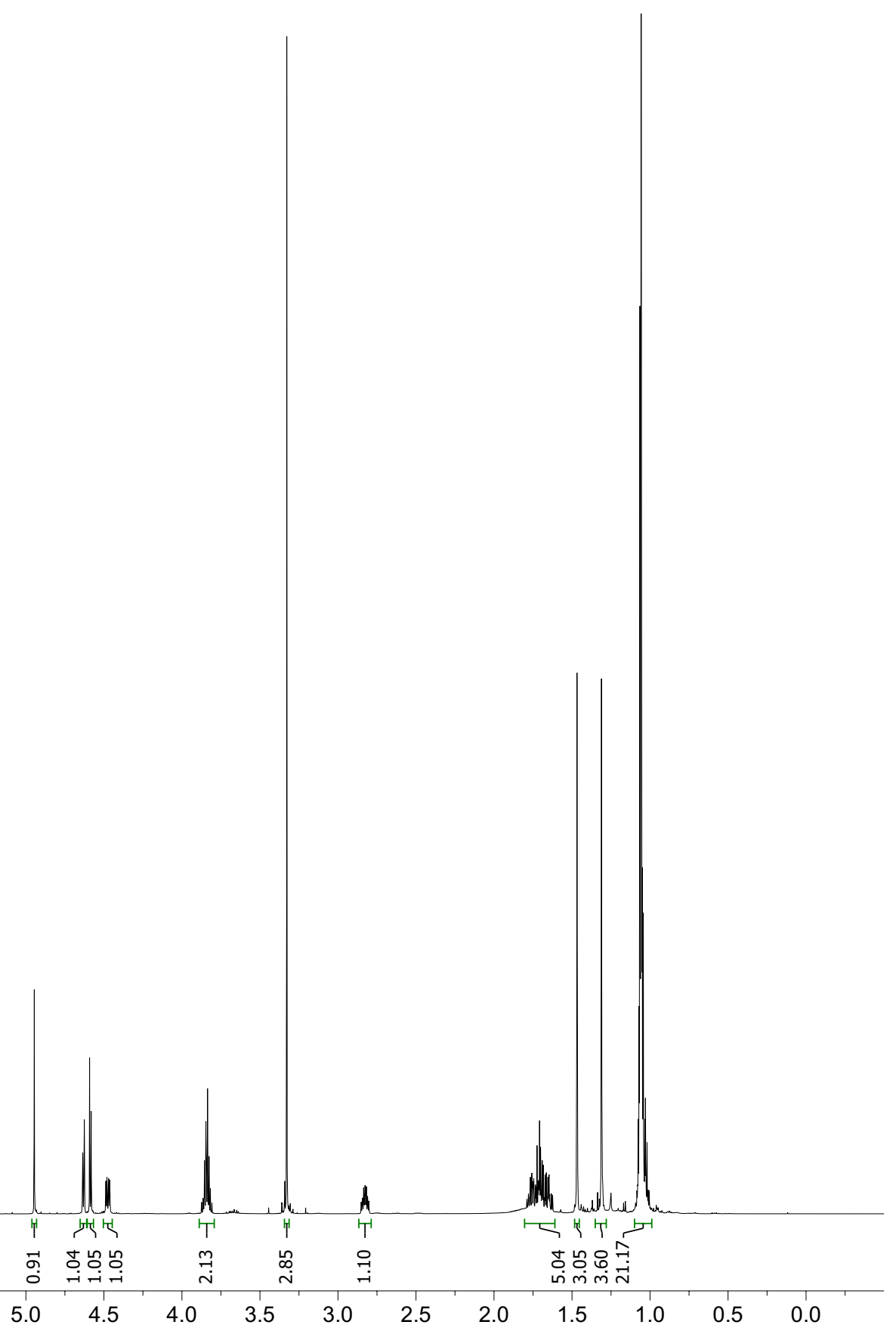




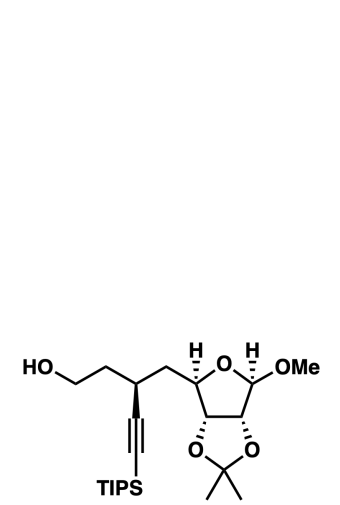

S52, ${ }^{13} \mathrm{C}, 151 \mathrm{MHz}, \mathrm{CDCl}_{3}$

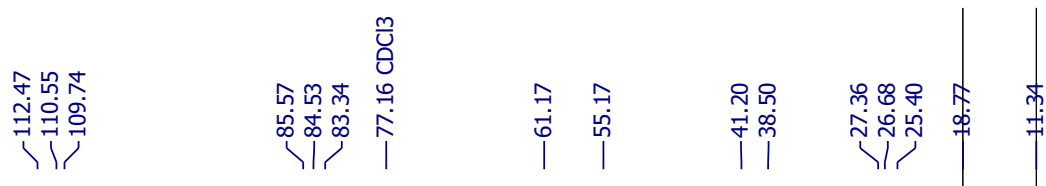

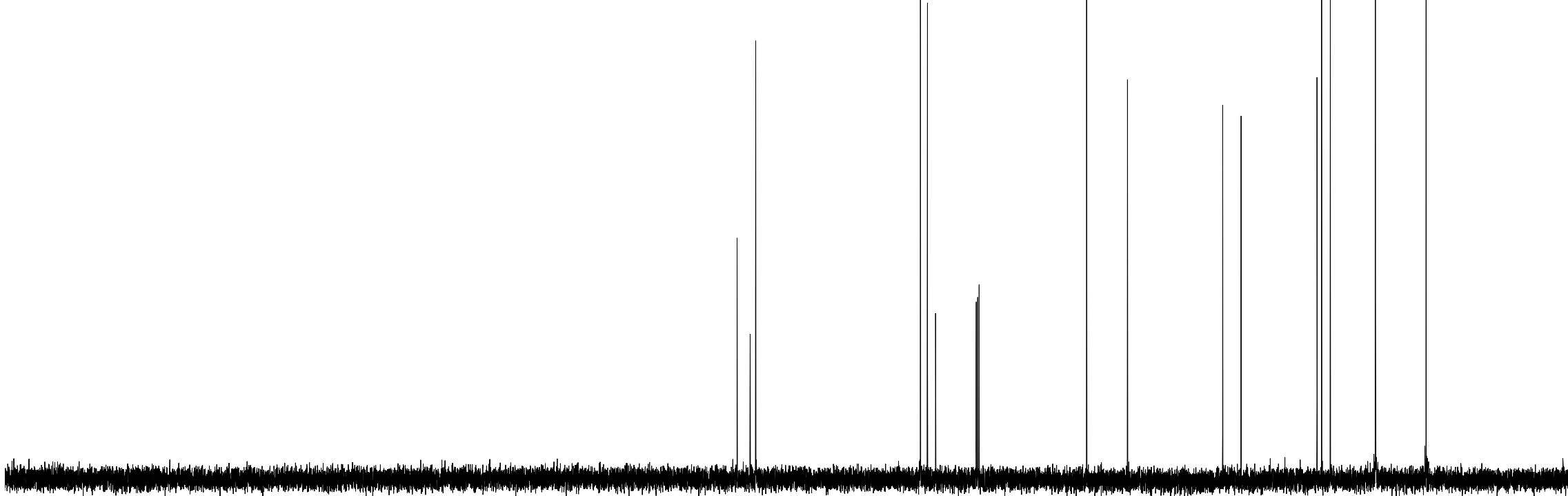




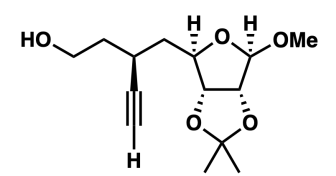

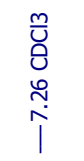

S53, ${ }^{1} \mathrm{H}, 500 \mathrm{MHz}, \mathrm{CDCl}_{3}$

虽

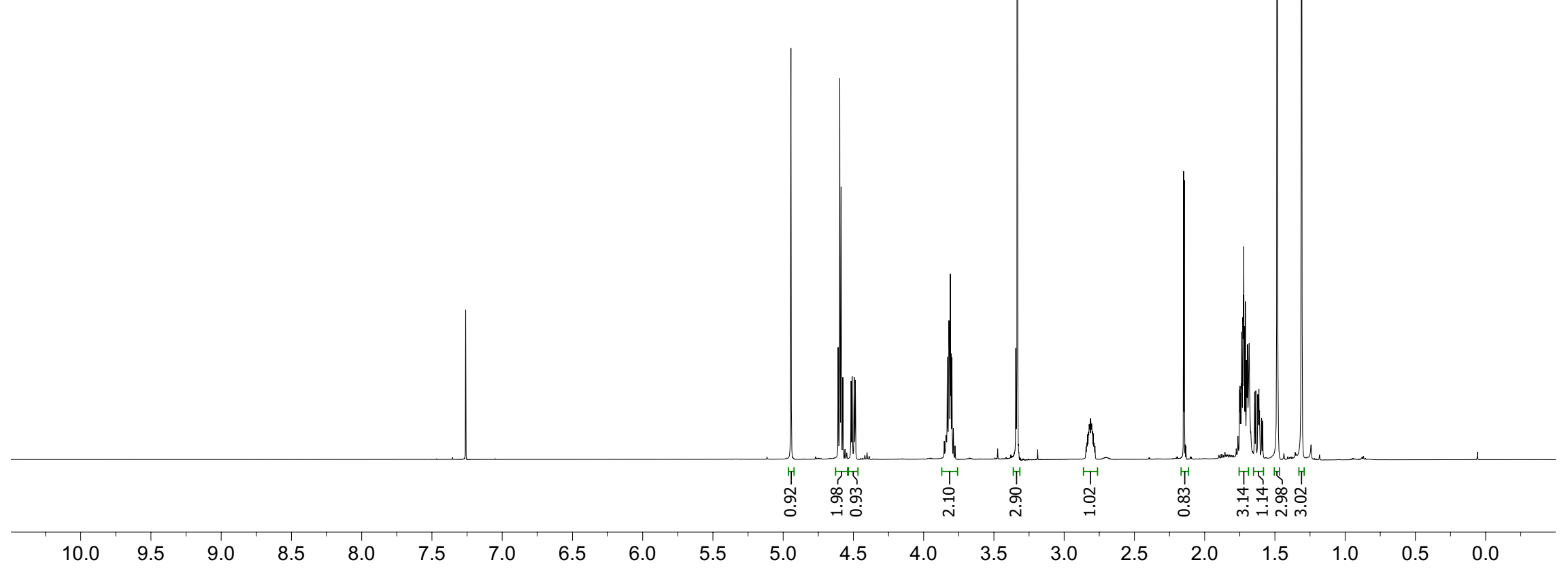




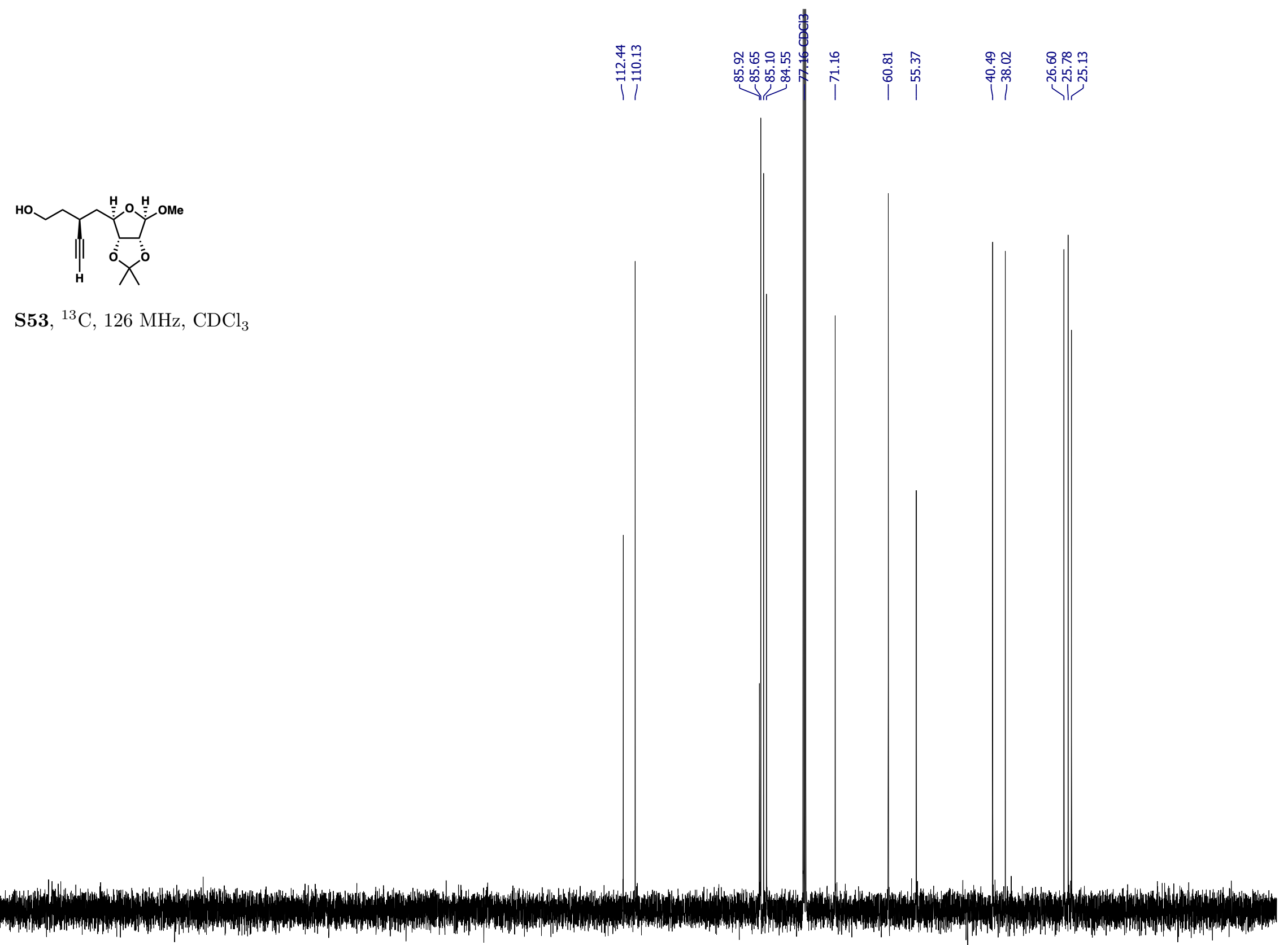

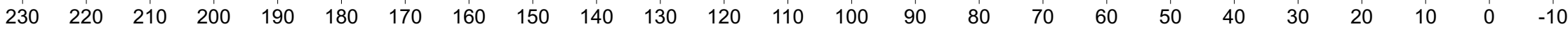




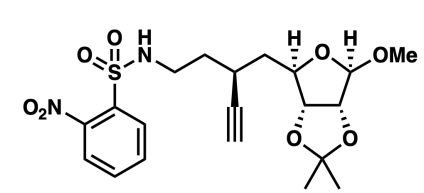

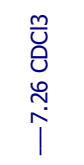

$\mathbf{S 5 4},{ }^{1} \mathrm{H}, 600 \mathrm{MHz}, \mathrm{CDCl}_{3}$

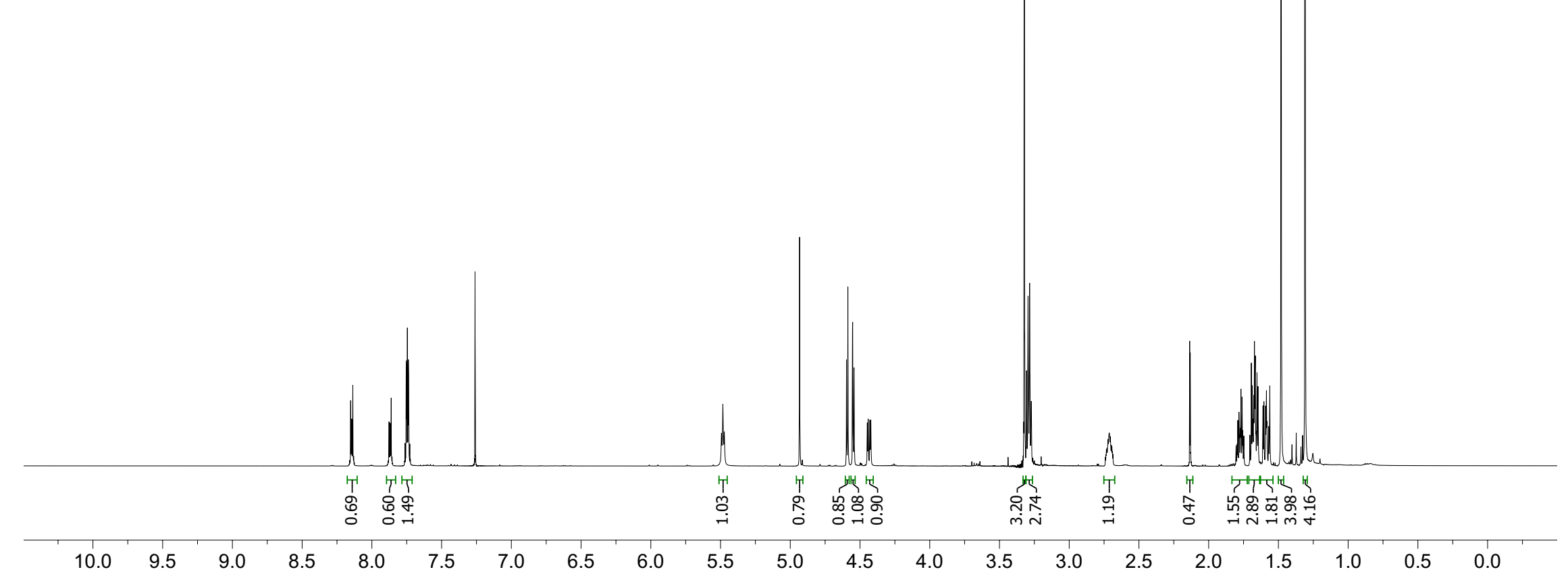




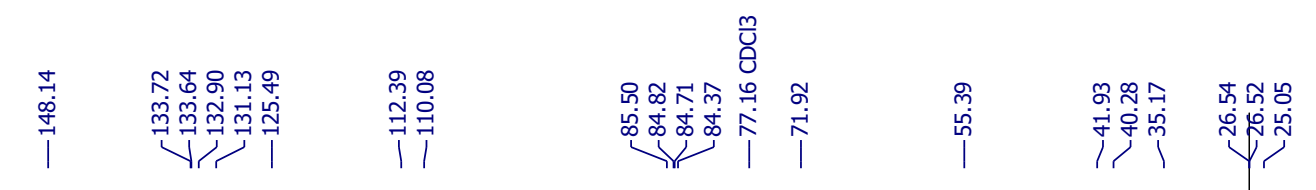

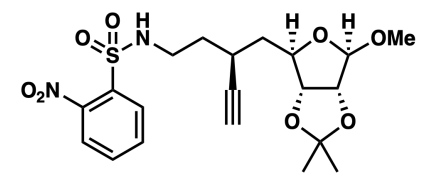

S54, ${ }^{13} \mathrm{C}, 151 \mathrm{MHz}, \mathrm{CDCl}_{3}$ 


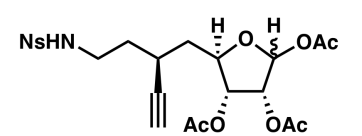

S55, ${ }^{1} \mathrm{H}, 600 \mathrm{MHz}, \mathrm{CDCl}_{3}$

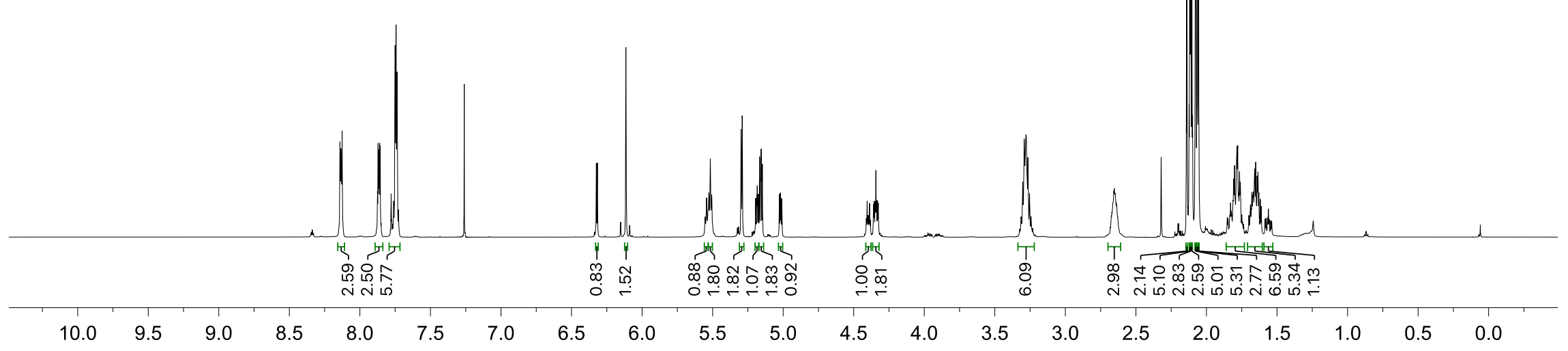




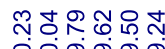

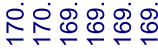

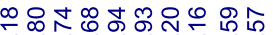

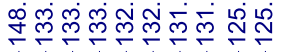

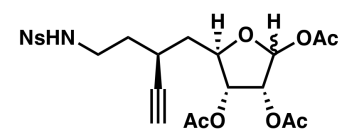

S55, ${ }^{13} \mathrm{C}, 126 \mathrm{MHz}, \mathrm{CDCl}_{3}$
兴

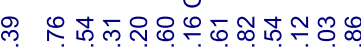

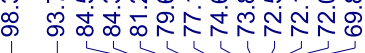

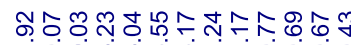

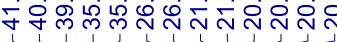




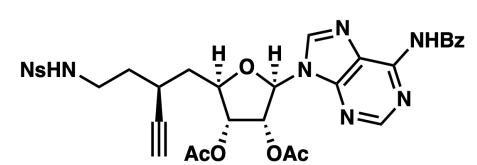

S56, ${ }^{1} \mathrm{H}, 600 \mathrm{MHz}, \mathrm{CDCl}_{3}$

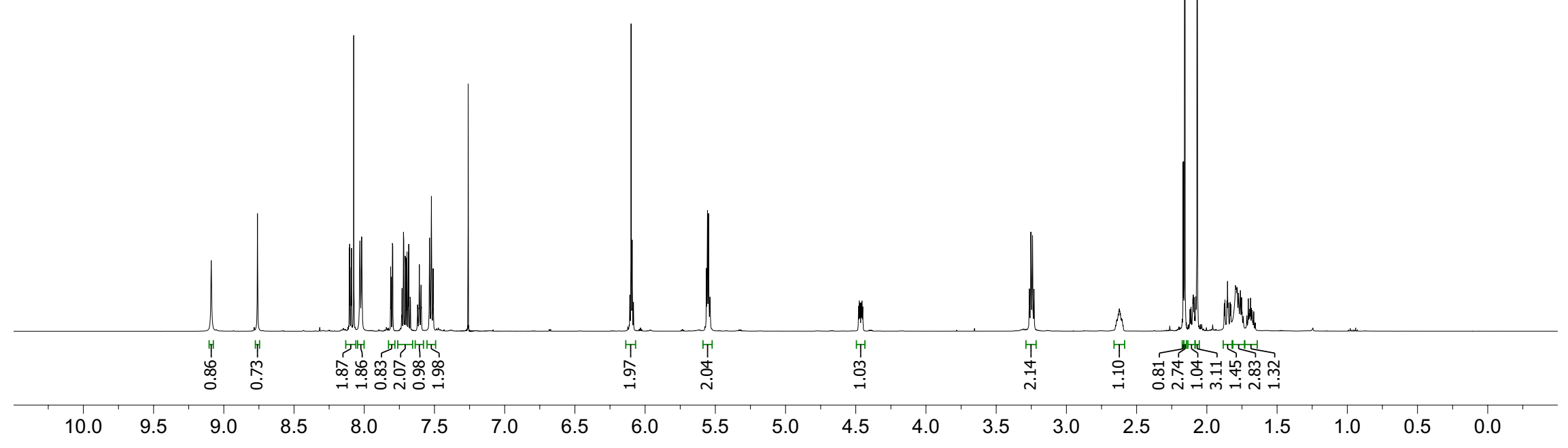




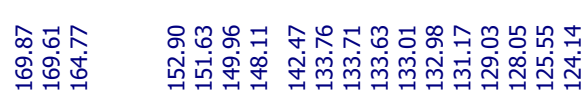

Vi रां।

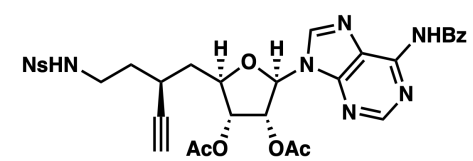

S56, ${ }^{13} \mathrm{C}, 101 \mathrm{MHz}, \mathrm{CDCl}_{3}$
苟|

궁ำ

ondising

잉요 웅

iा i 


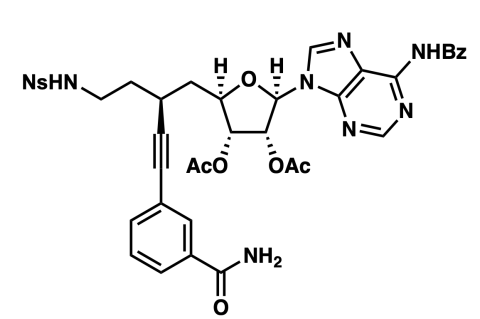

S57, ${ }^{1} \mathrm{H}, 500 \mathrm{MHz}, \mathrm{CDCl}_{3}$

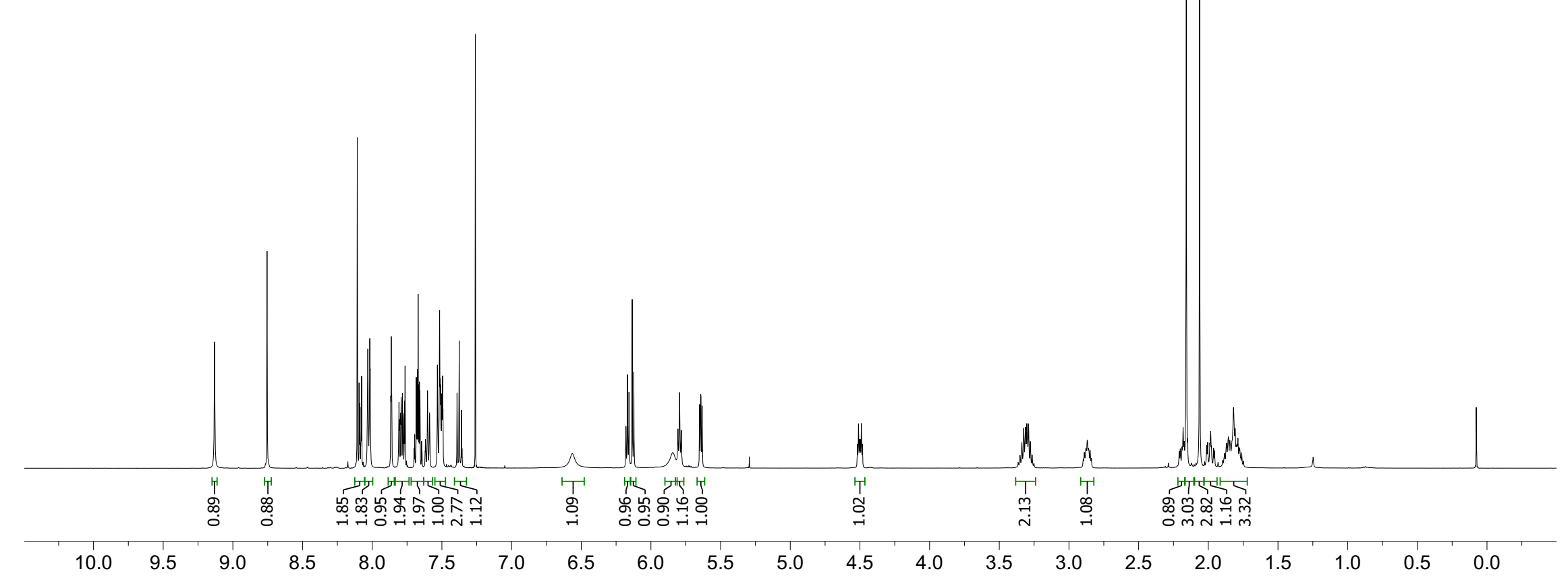




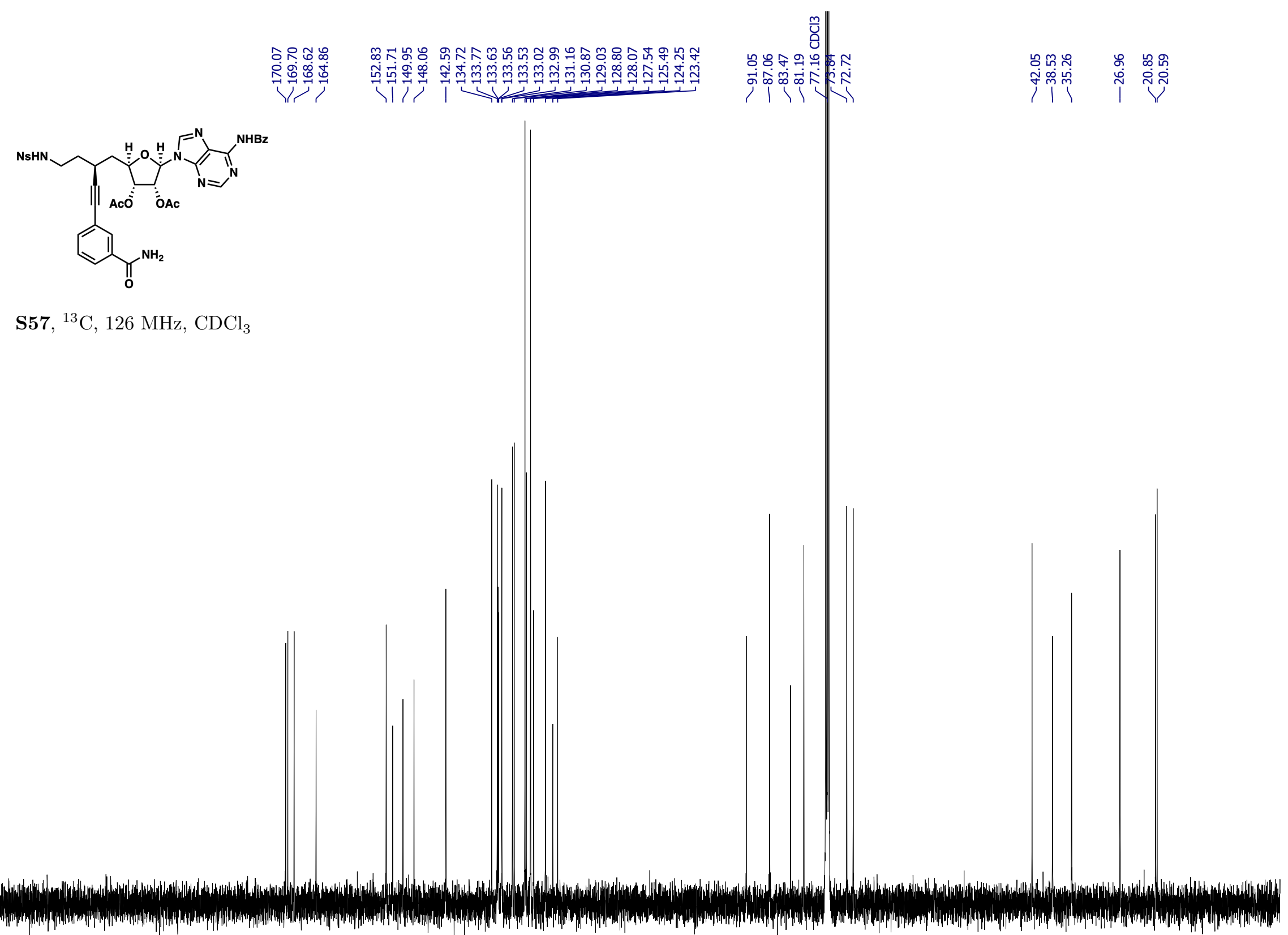

$210 \quad 200$

$\begin{array}{lll}180 & 170 \quad 160\end{array}$

$150 \quad 140$

130

120

$100 \quad 90$
0

$80 \quad 70 \quad 60$

50

$40 \quad 30$

$20 \quad 10 \quad 0$ 


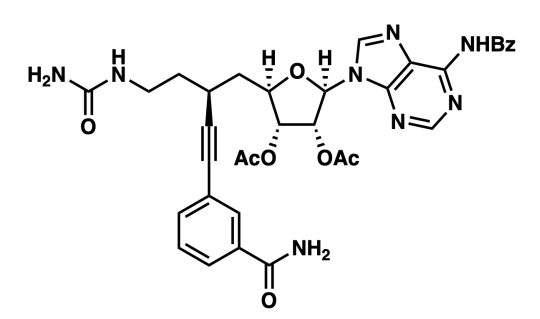

S58, ${ }^{1} \mathrm{H}, 600 \mathrm{MHz}, \mathrm{CD}_{3} \mathrm{OD}$

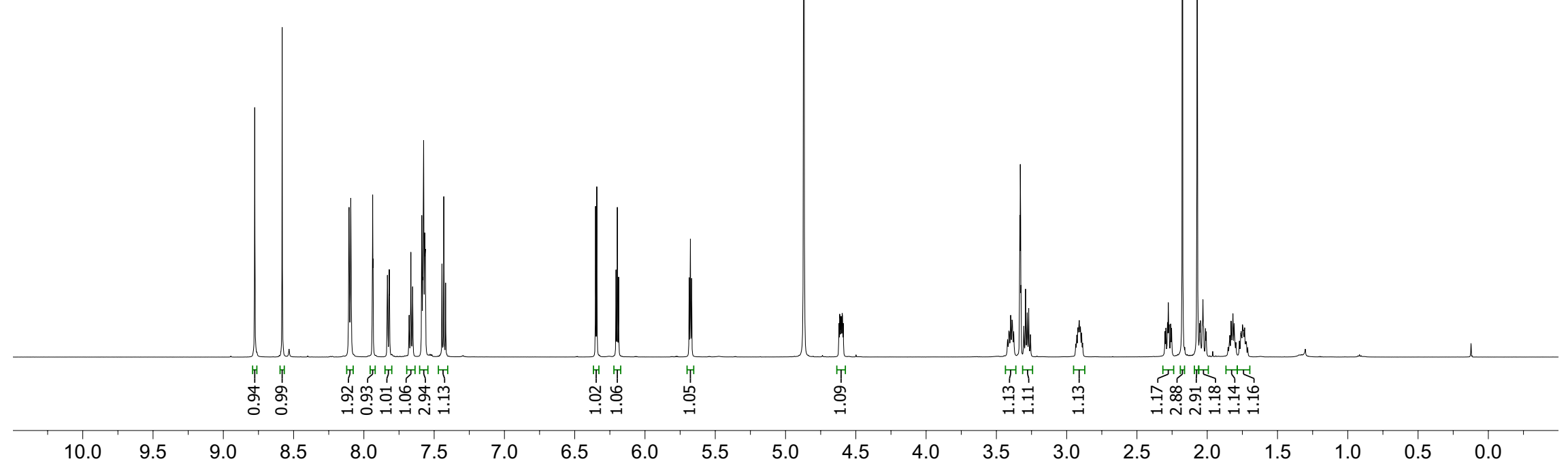




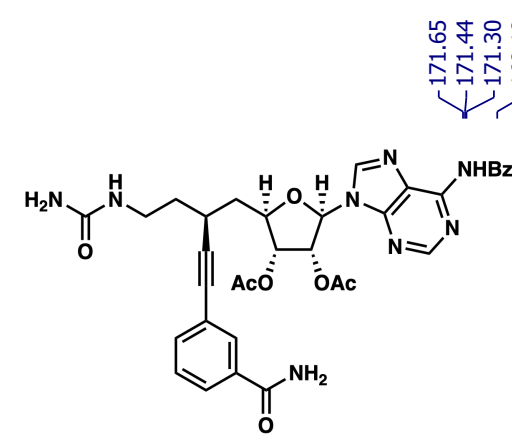

S58, ${ }^{13} \mathrm{C}, 126 \mathrm{MHz}, \mathrm{CD}_{3} \mathrm{OD}$

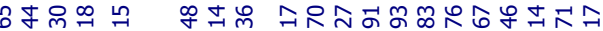

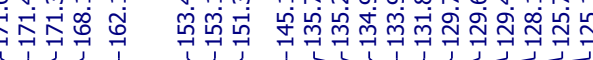

NHBz

।

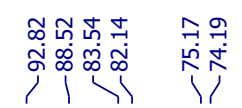

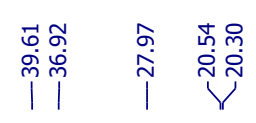

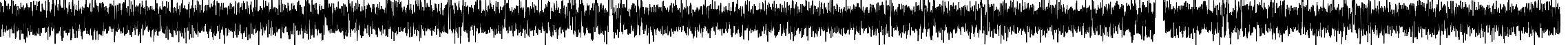

210200

$190 \quad 180$

$170 \quad 160$

$150 \quad 140$

130

20

100

90

80

70

$60 \quad 50$

$40 \quad 30$

$20 \quad 10$ 


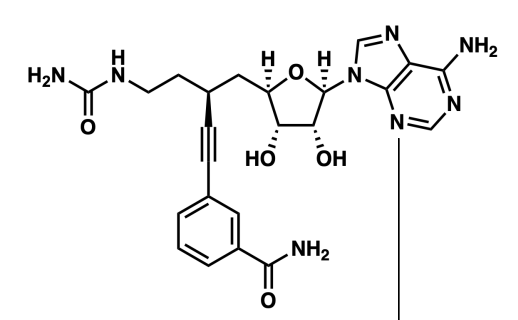

25, ${ }^{1} \mathrm{H}, 600 \mathrm{MHz}, \mathrm{CD}_{3} \mathrm{CN} / \mathbf{D}_{\mathbf{2}} \mathbf{O} / d-\mathrm{TFA}$

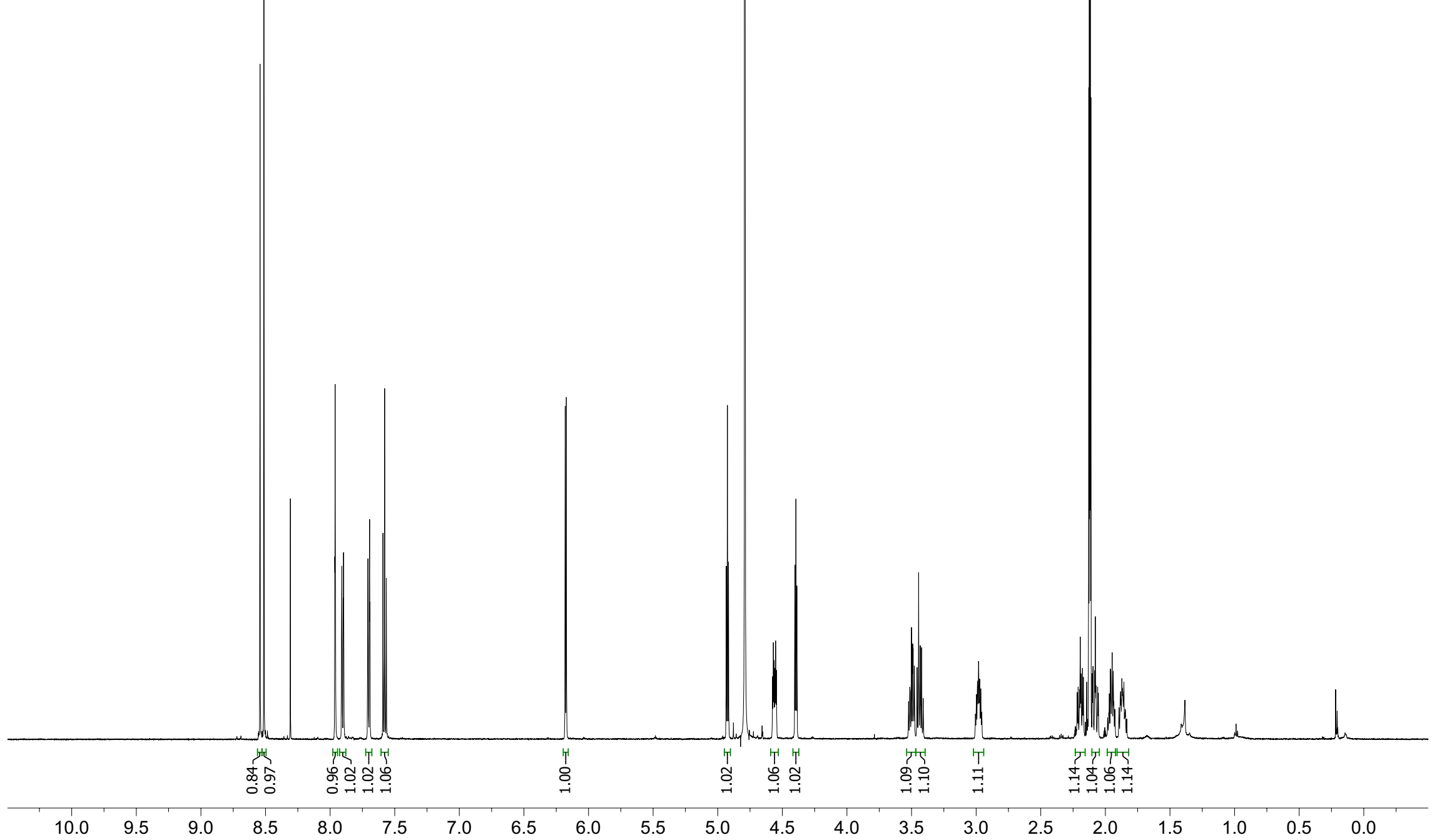




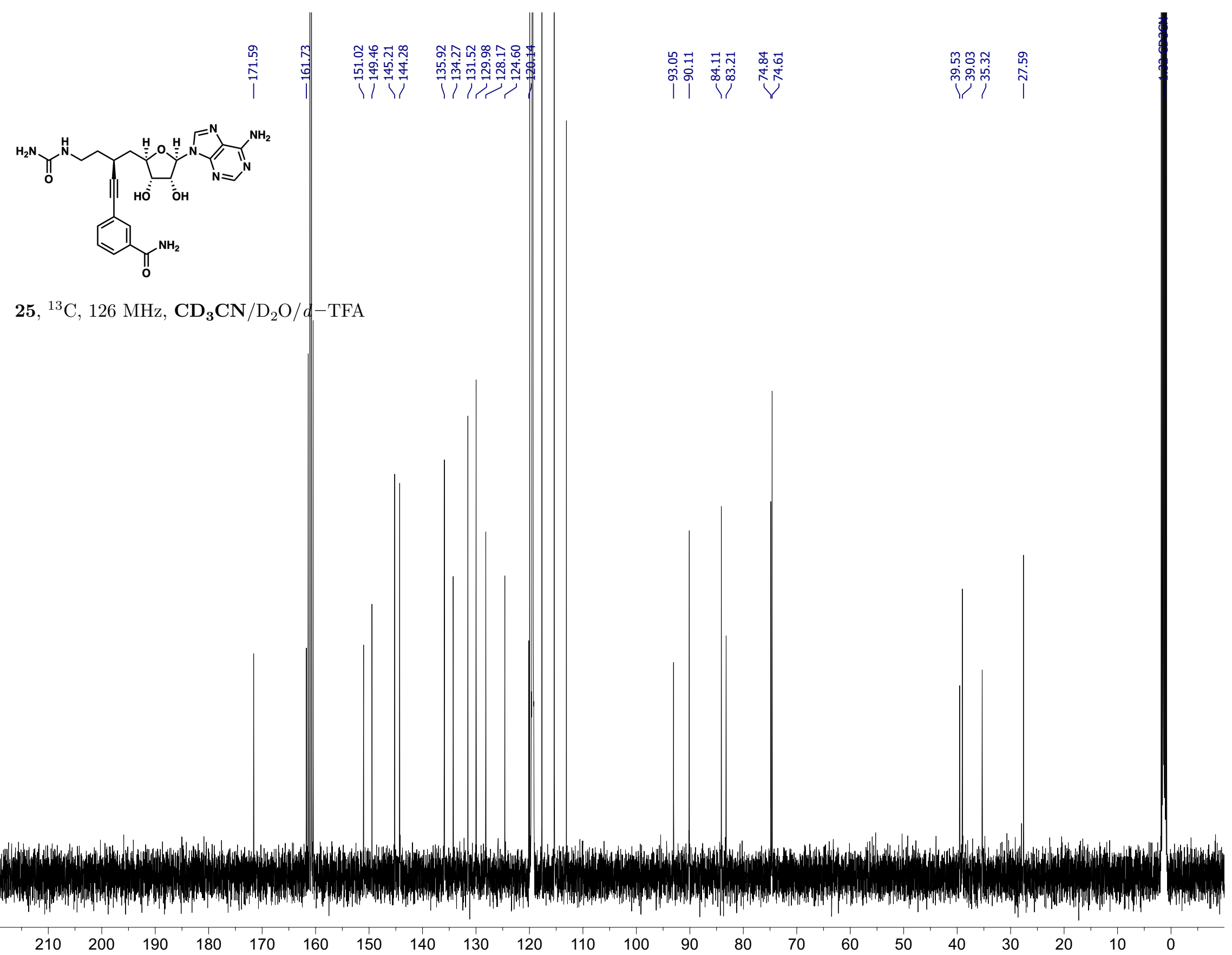




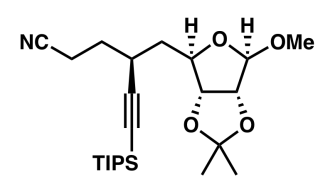

$\mathbf{S 5 9},{ }^{1} \mathrm{H}, 500 \mathrm{MHz}, \mathrm{CDCl}_{3}$

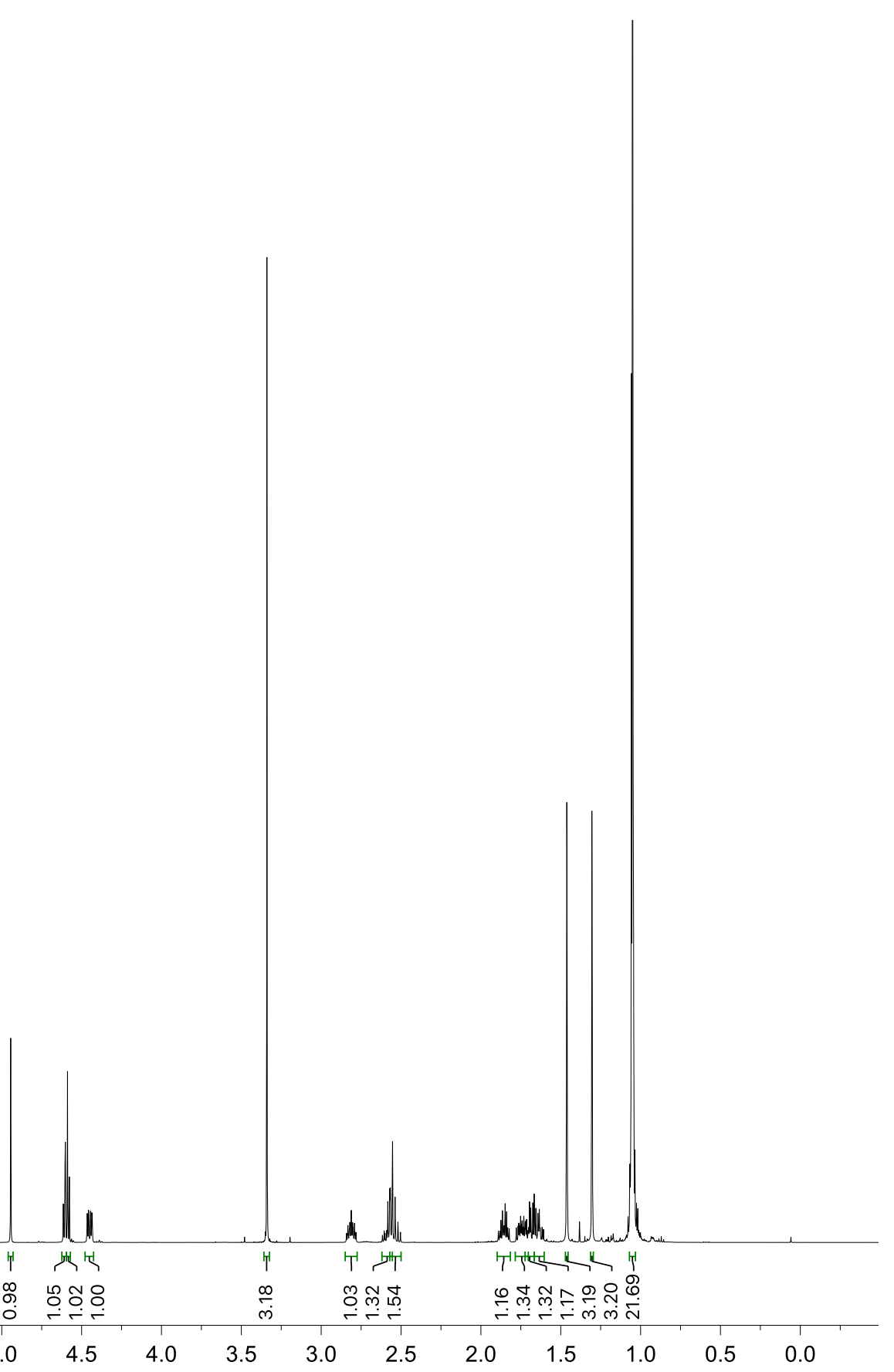




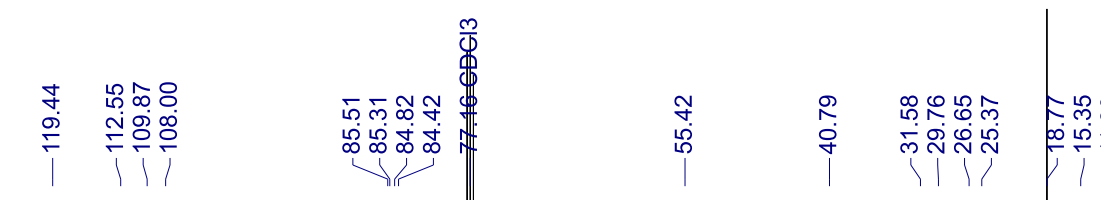

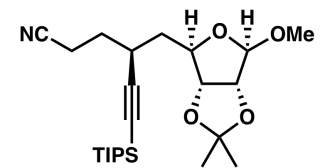

S59, ${ }^{13} \mathrm{C}, 100 \mathrm{MHz}, \mathrm{CDCl}_{3}$

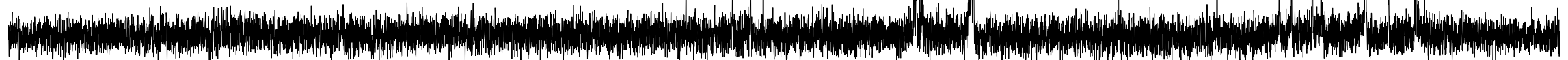




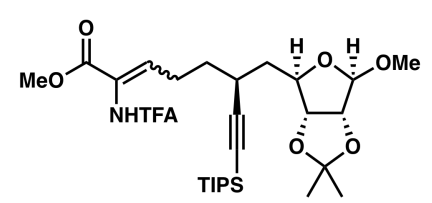

S61, ${ }^{1} \mathrm{H}, 500 \mathrm{MHz}, \mathrm{CDCl}_{3}$

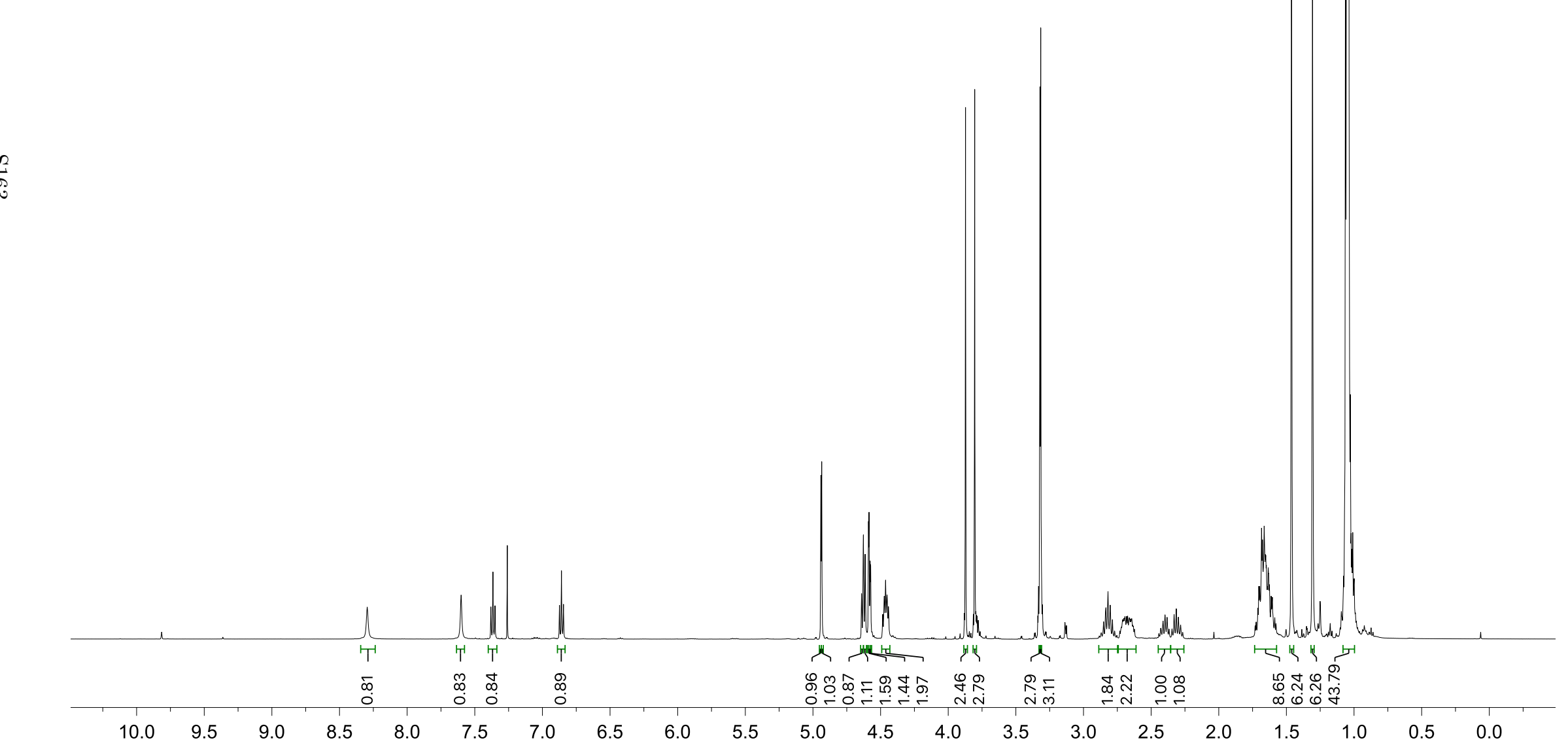




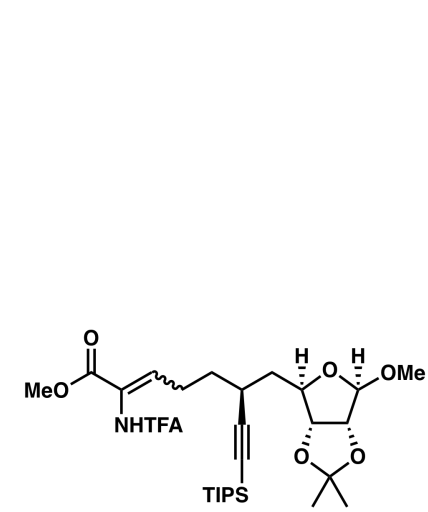

ᄂூ

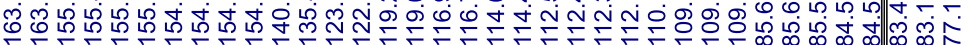

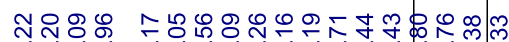

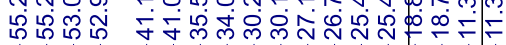

S61, ${ }^{13} \mathrm{C}, 126 \mathrm{MHz}, \mathrm{CDCl}_{3}$ 


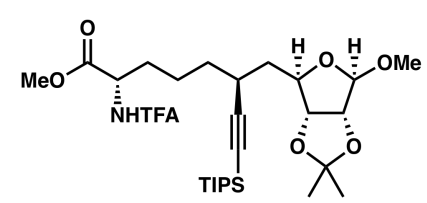

S62, ${ }^{1} \mathrm{H}, 500 \mathrm{MHz}, \mathrm{CDCl}_{3}$

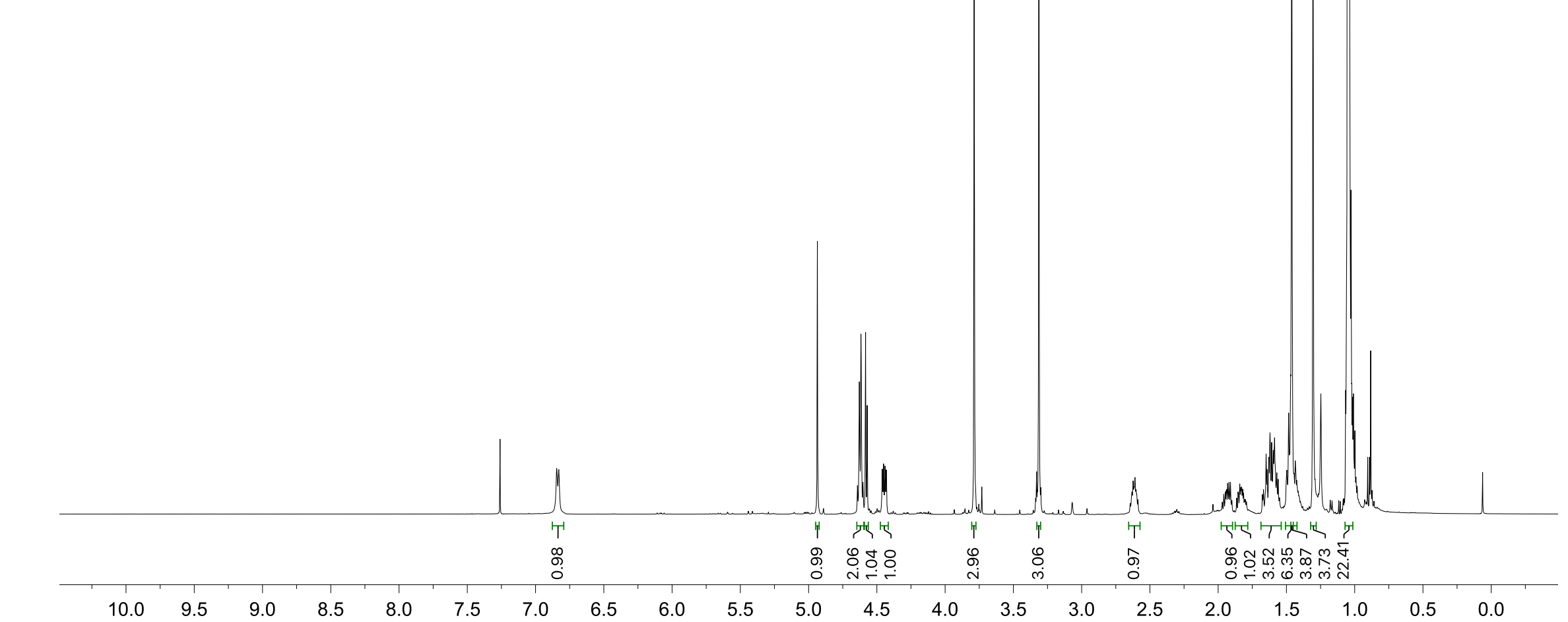




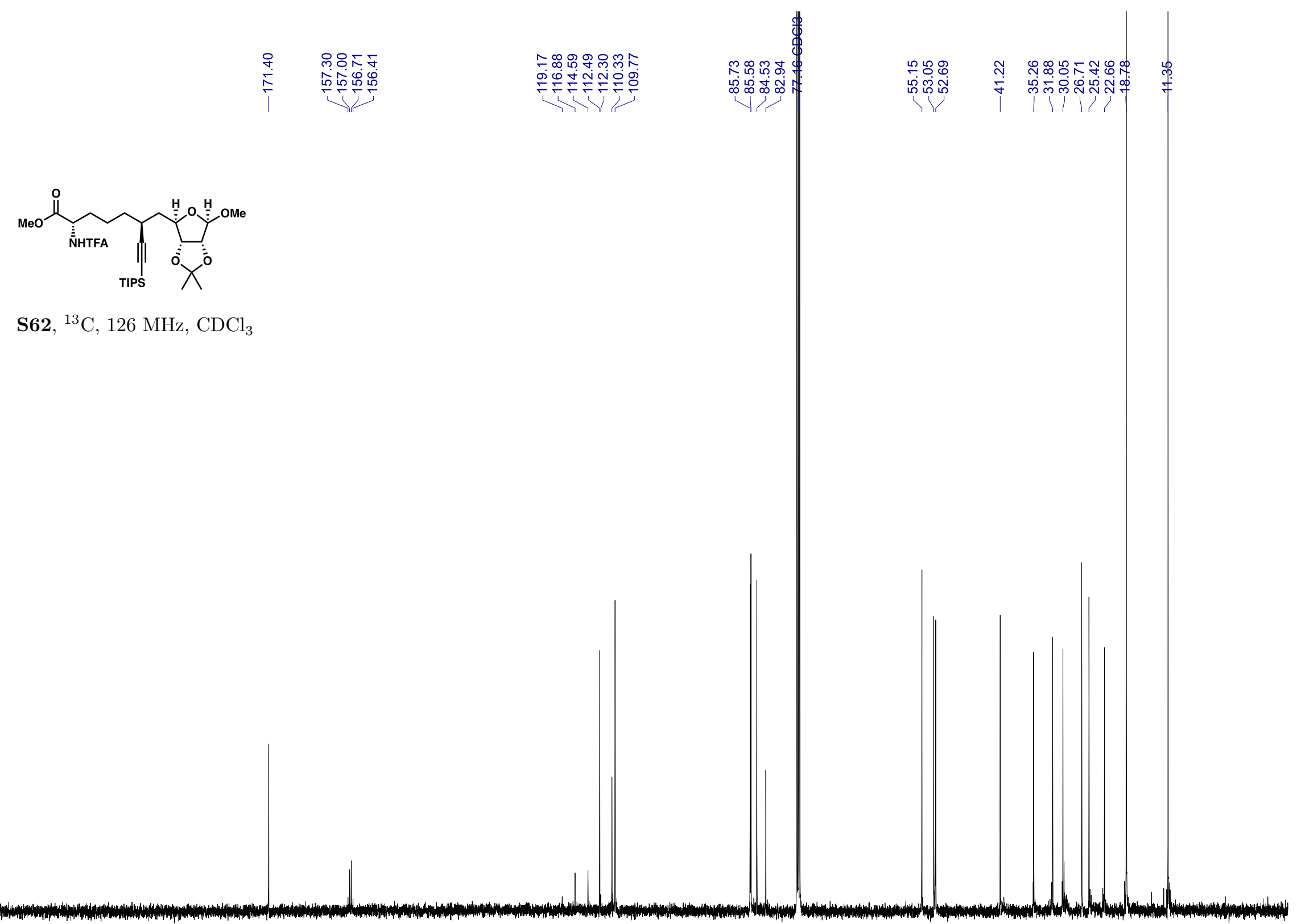

$210 \quad 200$

190

180

170

160

150

140

130

120

110

100

90

80

70

60

50

$40 \quad 30$

20

10 0 


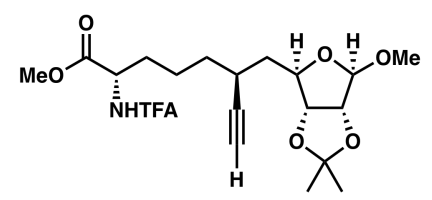

S63, ${ }^{1} \mathrm{H}, 500 \mathrm{MHz}, \mathrm{CDCl}_{3}$ 


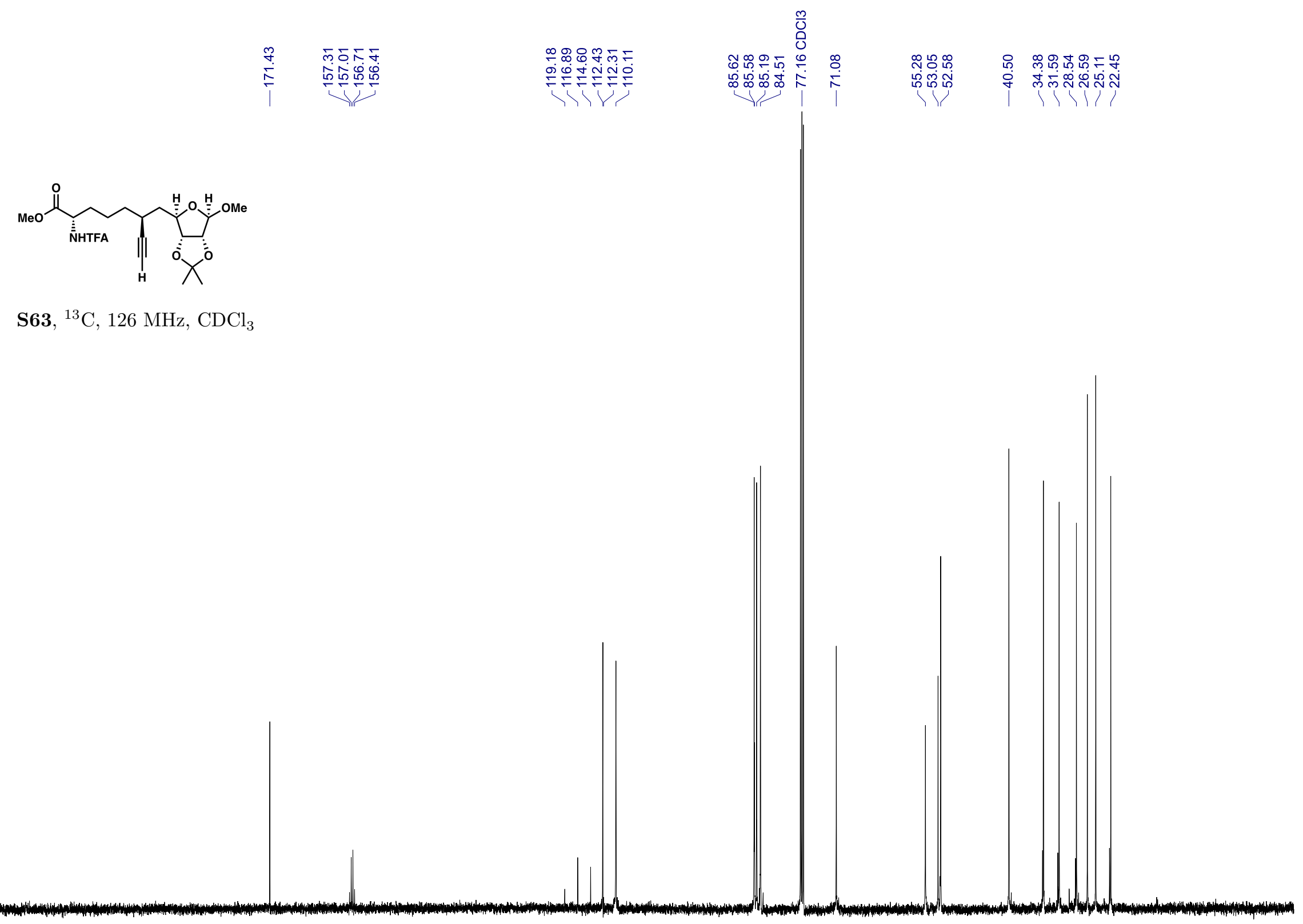




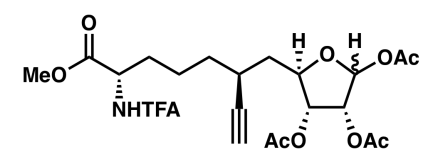

S64, ${ }^{1} \mathrm{H}, 500 \mathrm{MHz}, \mathrm{CDCl}_{3}$

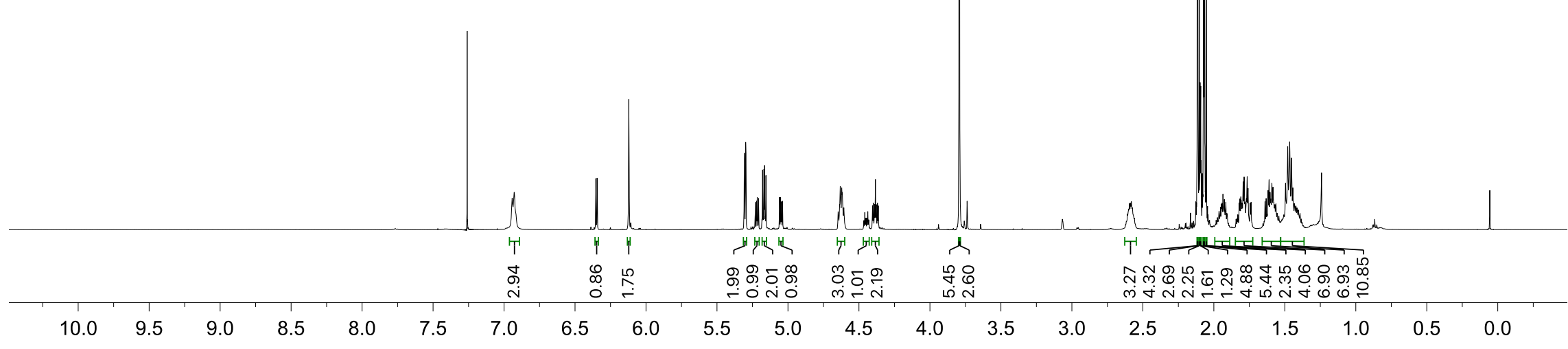




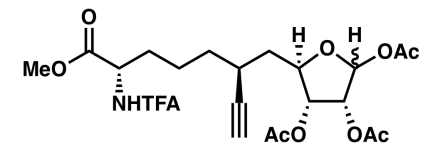

S64, ${ }^{13} \mathrm{C}, 126 \mathrm{MHz}, \mathrm{CDCl}_{3}$
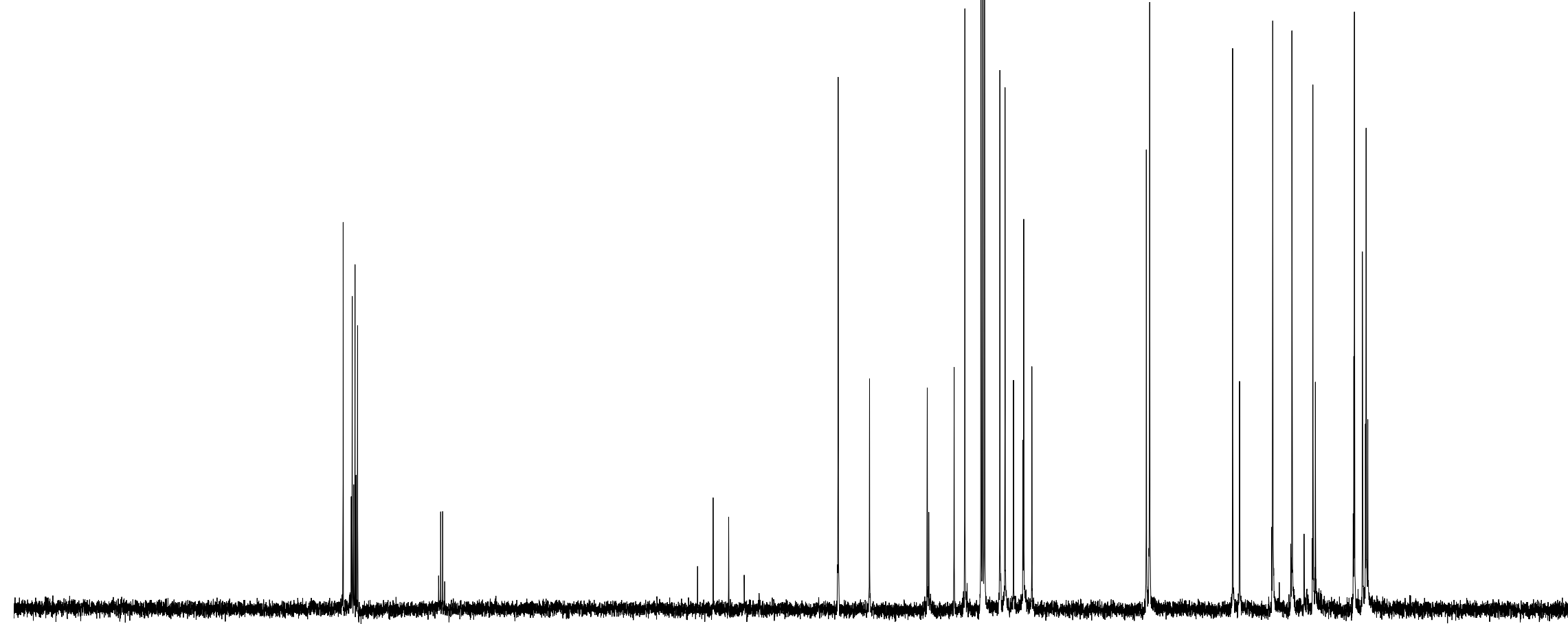


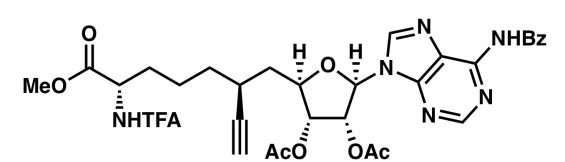

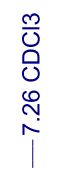

S65, ${ }^{1} \mathrm{H}, 600 \mathrm{MHz}, \mathrm{CDCl}_{3}$

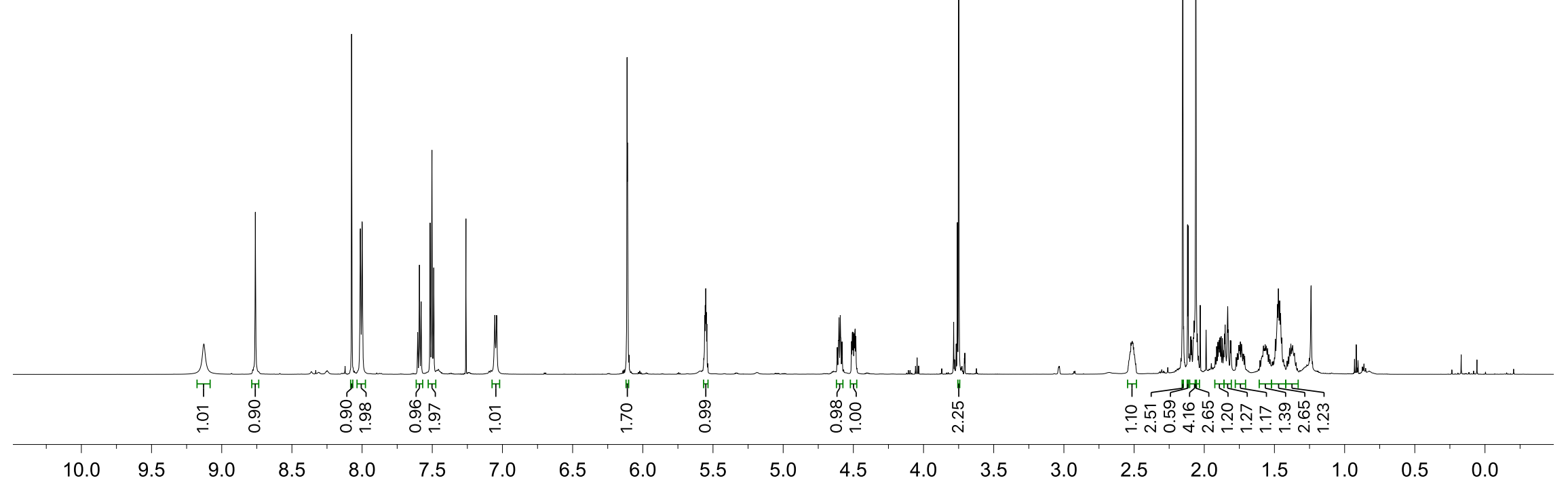




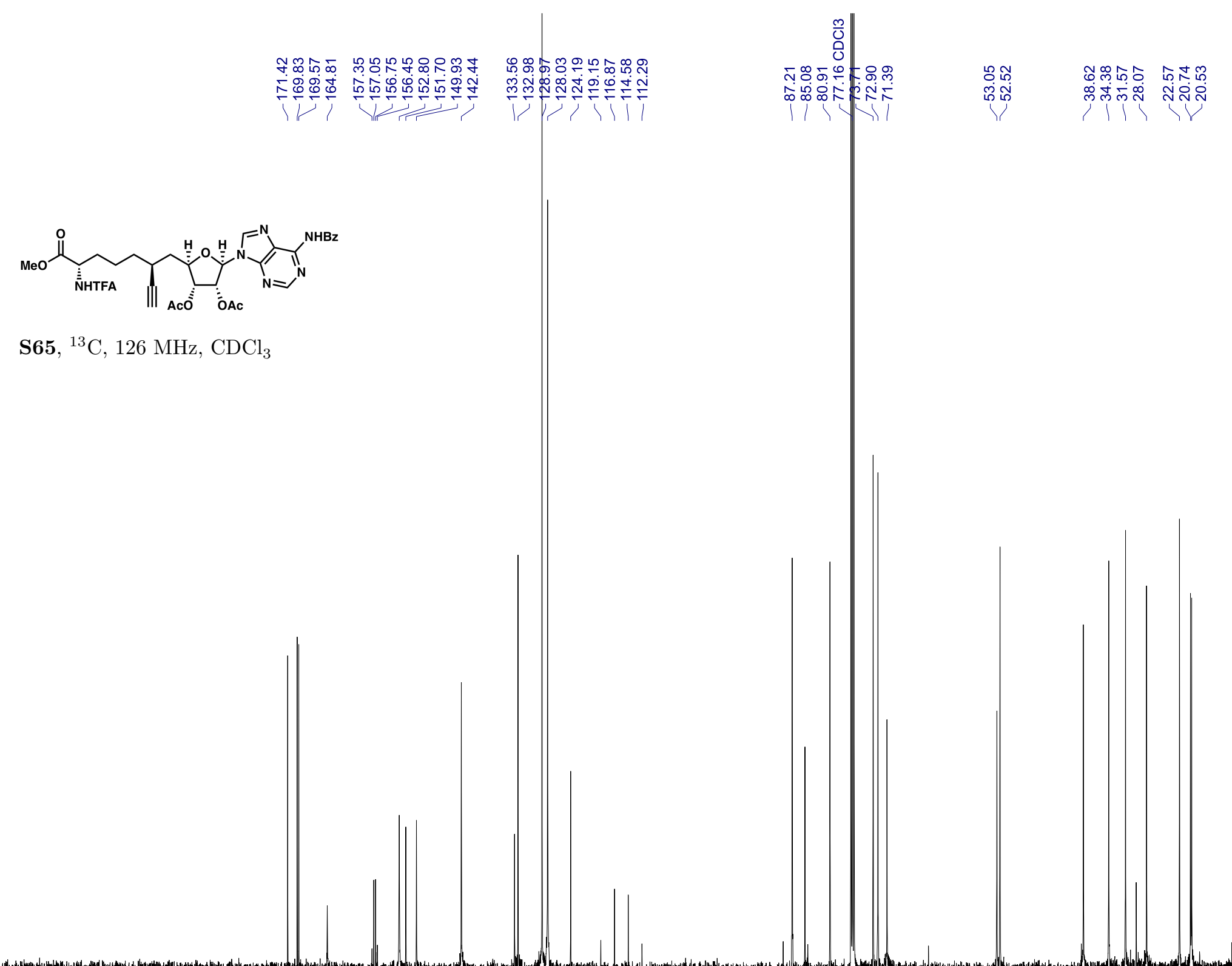

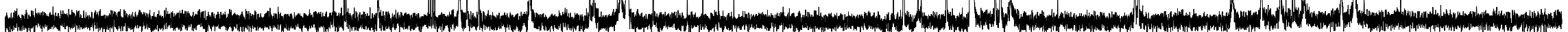




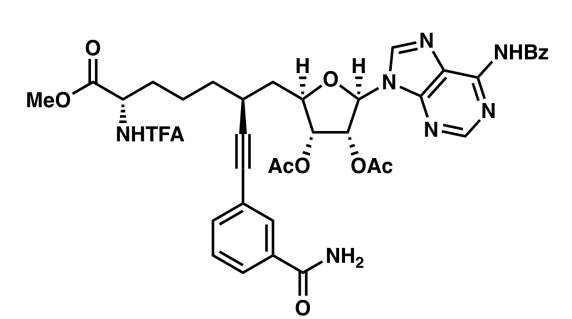

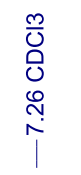

S66, ${ }^{1} \mathrm{H}, 500 \mathrm{MHz}, \mathrm{CDCl}_{3}$

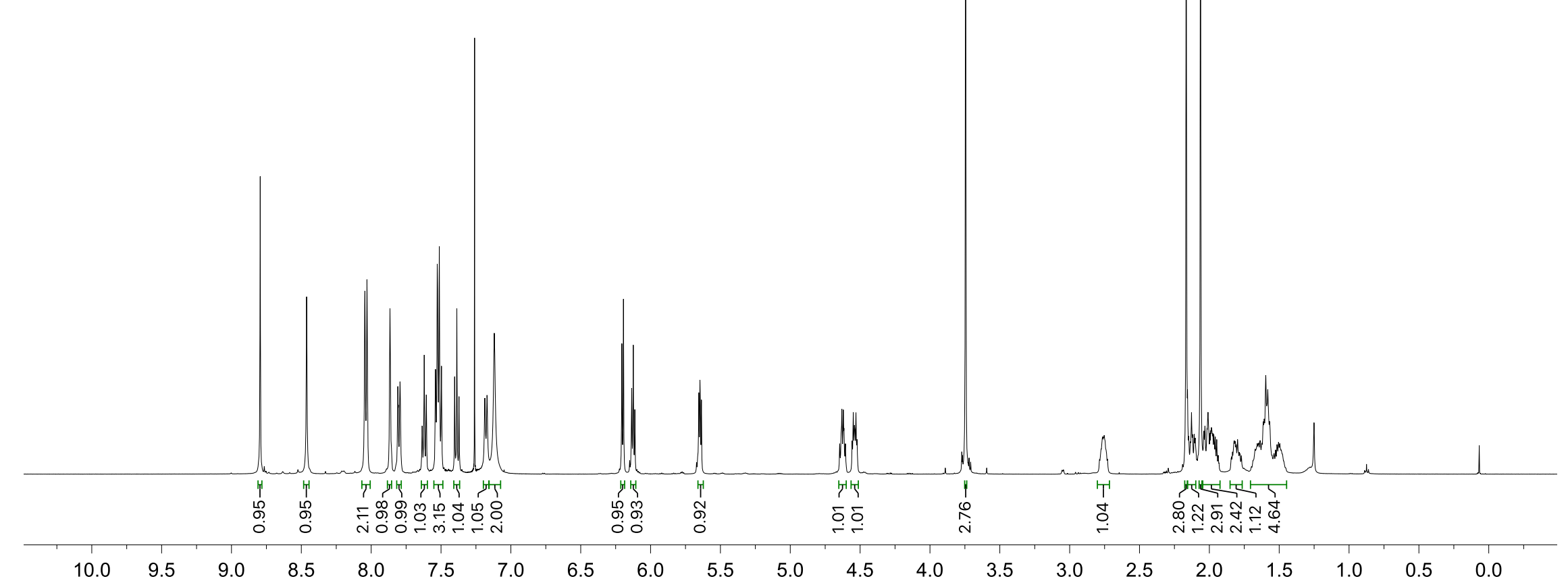




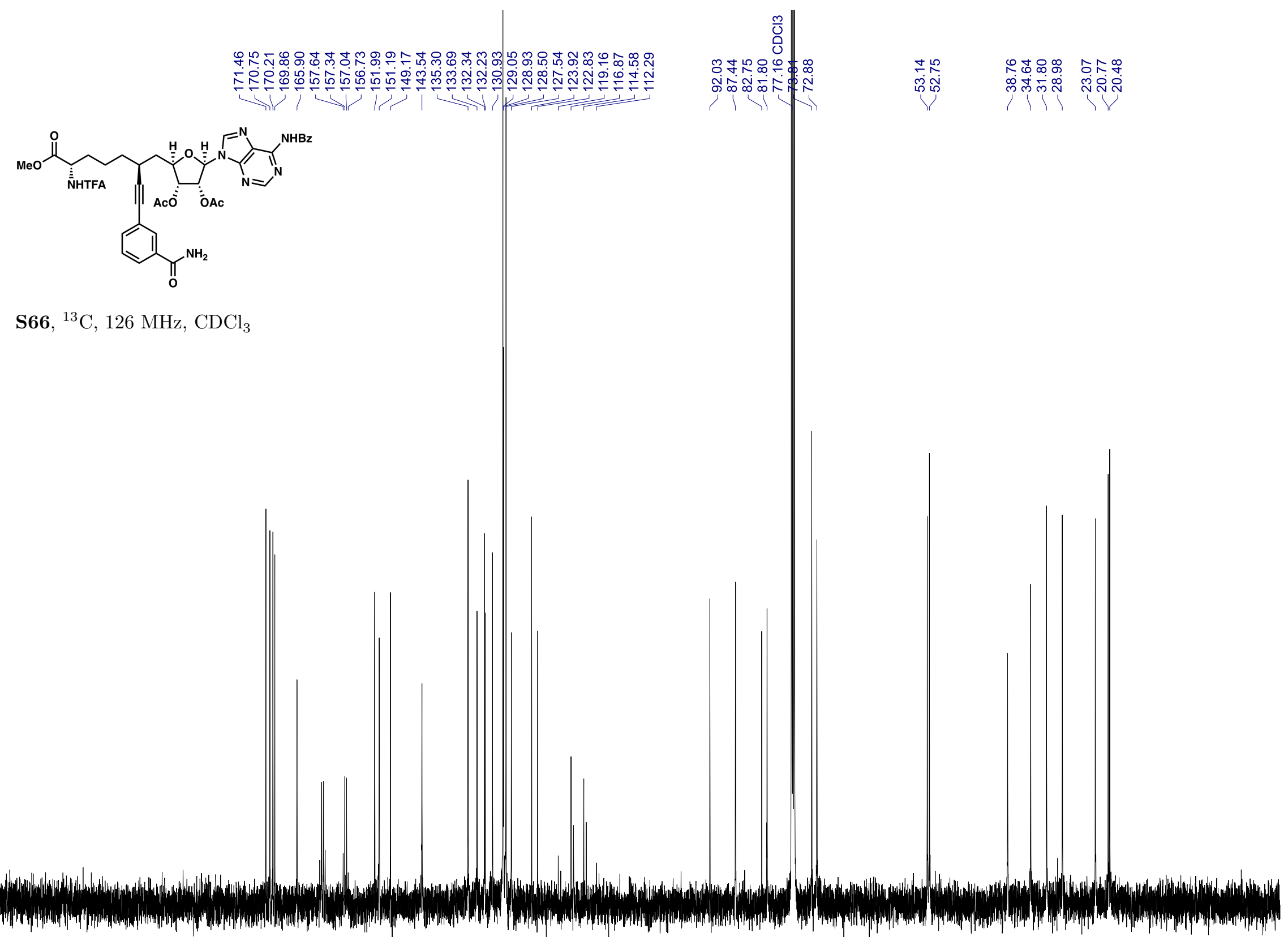

$210 \quad 200$

190

180

$170 \quad 160$

150

140

130

120

$110 \quad 100$

90

$80 \quad 70$

60

50

$40 \quad 30$

$20 \quad 10$ 0 


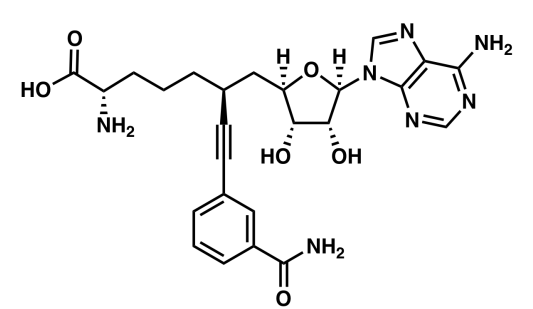

26, ${ }^{1} \mathrm{H}, 600 \mathrm{MHz}, \mathrm{D}_{2} \mathrm{O}$

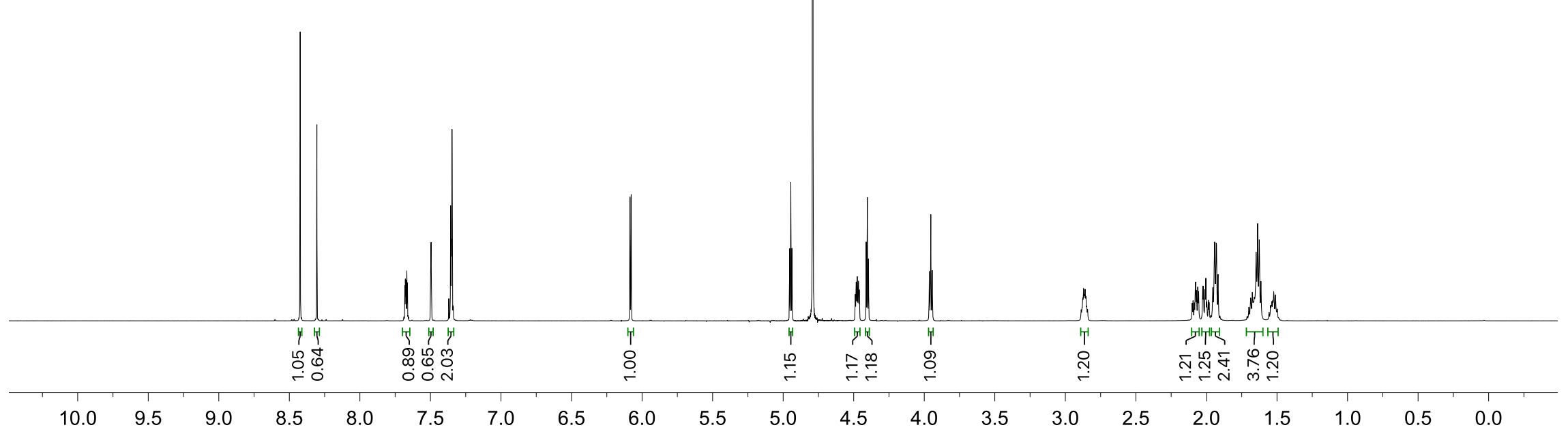




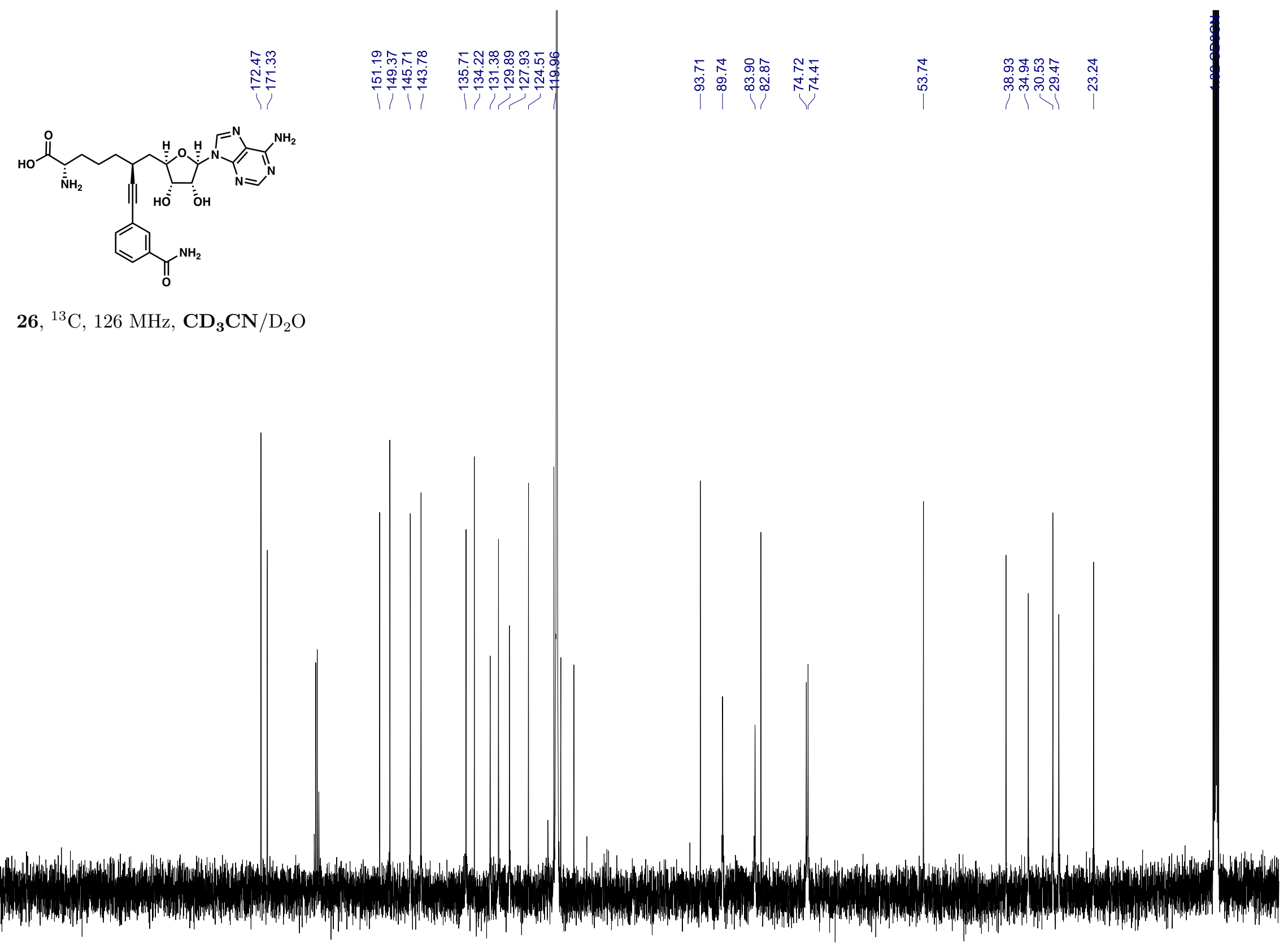

(3) 


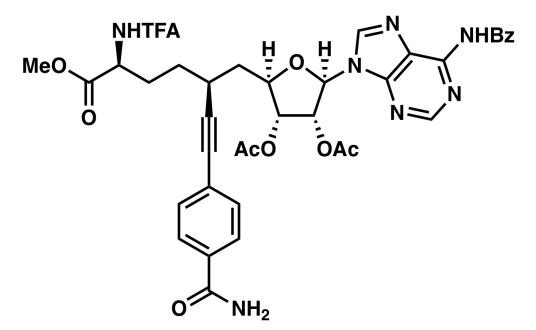

S67, ${ }^{1} \mathrm{H}, 500 \mathrm{MHz}, \mathrm{CD}_{3} \mathrm{OD}$

$\stackrel{\sim}{\mathcal{V}}$

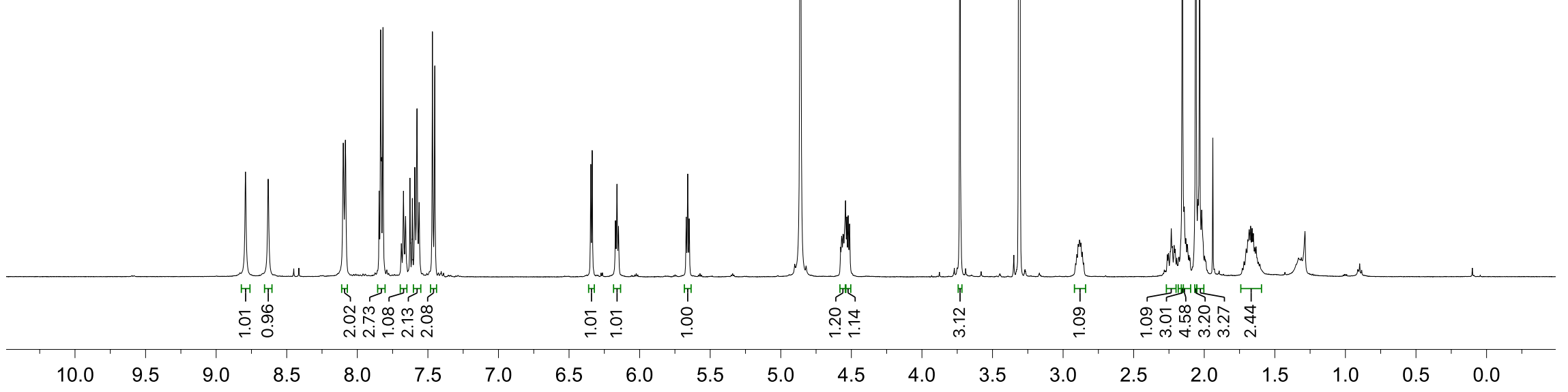




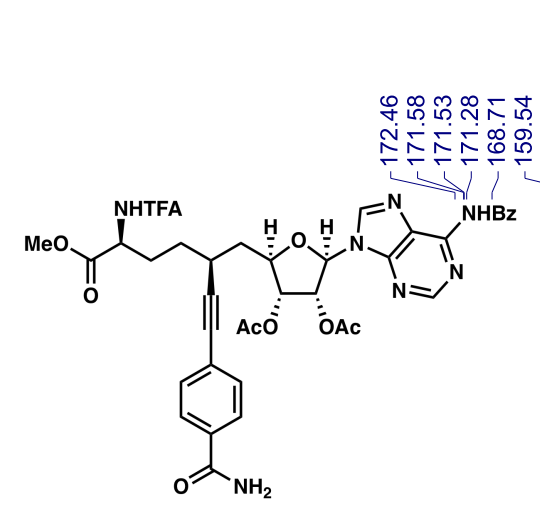

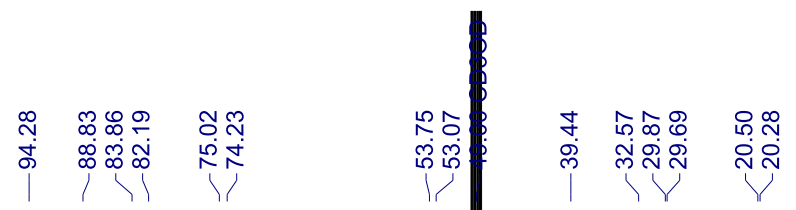

S67, ${ }^{13} \mathrm{C}, 126 \mathrm{MHz}, \mathrm{CD}_{3} \mathrm{OD}$

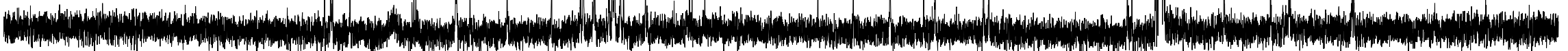




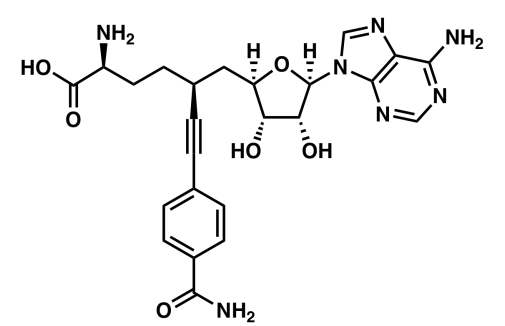

$z$
0
0
0
$\vdots$
$\dot{o}$
|

27, ${ }^{1} \mathrm{H}, 600 \mathrm{MHz}, \mathbf{C D}_{\mathbf{3}} \mathbf{C N} / \mathrm{D}_{2} \mathrm{O}$

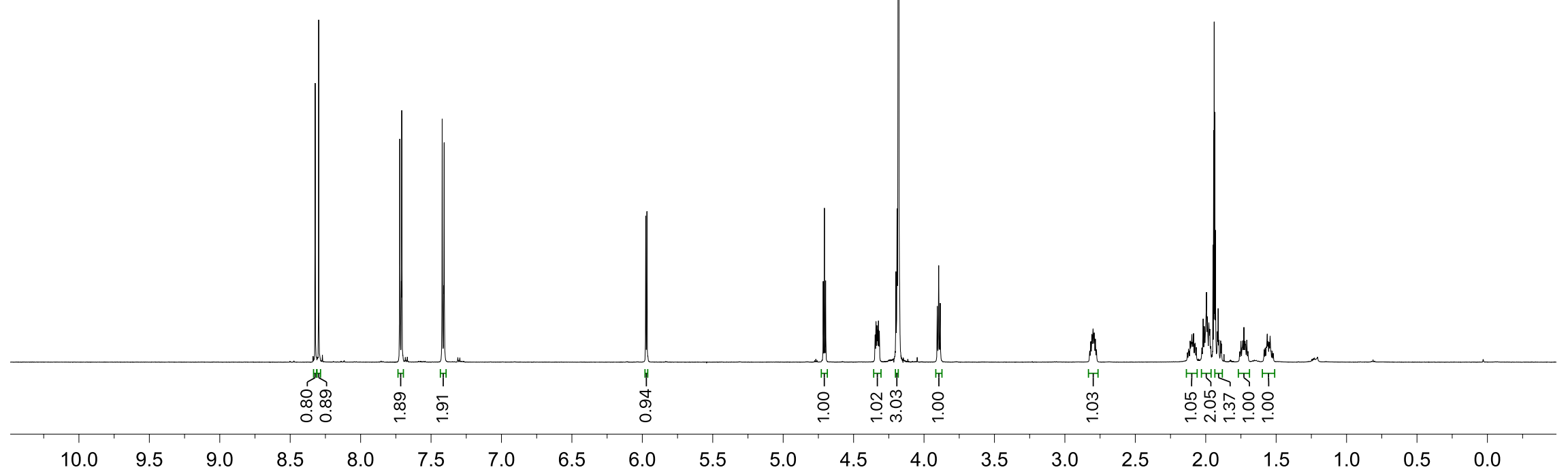




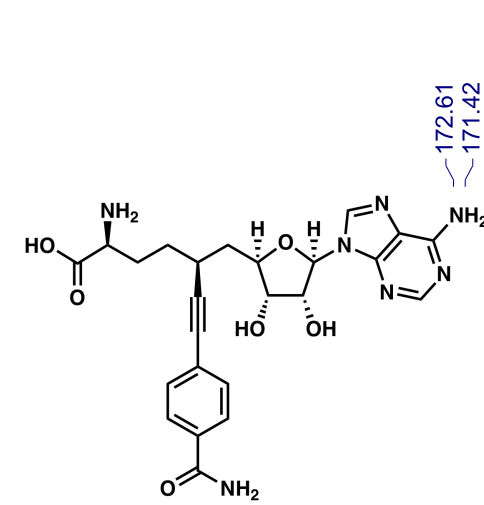

27, ${ }^{13} \mathrm{C}, 126 \mathrm{MHz}, \mathbf{C D}_{\mathbf{3}} \mathbf{C N} / \mathrm{D}_{2} \mathrm{O}$

에

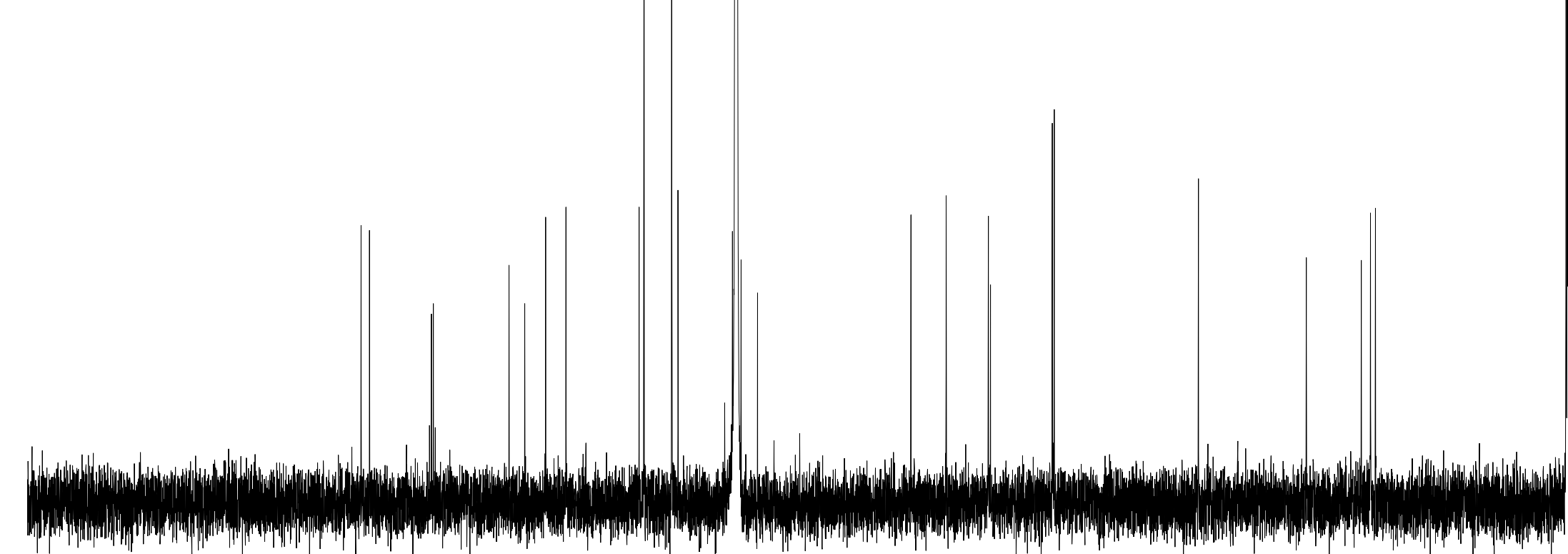

$210 \quad 200$

190

$\begin{array}{lll}180 & 170 & 160\end{array}$

150

130

120

$110 \quad 100$

90

80

70

$60 \quad 50$

$40 \quad 30$

20

10 


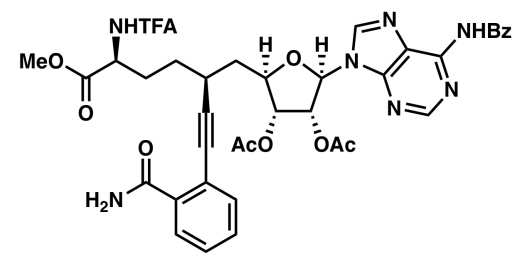

S68, ${ }^{1} \mathrm{H}, 600 \mathrm{MHz}, \mathrm{CD}_{3} \mathrm{OD}$

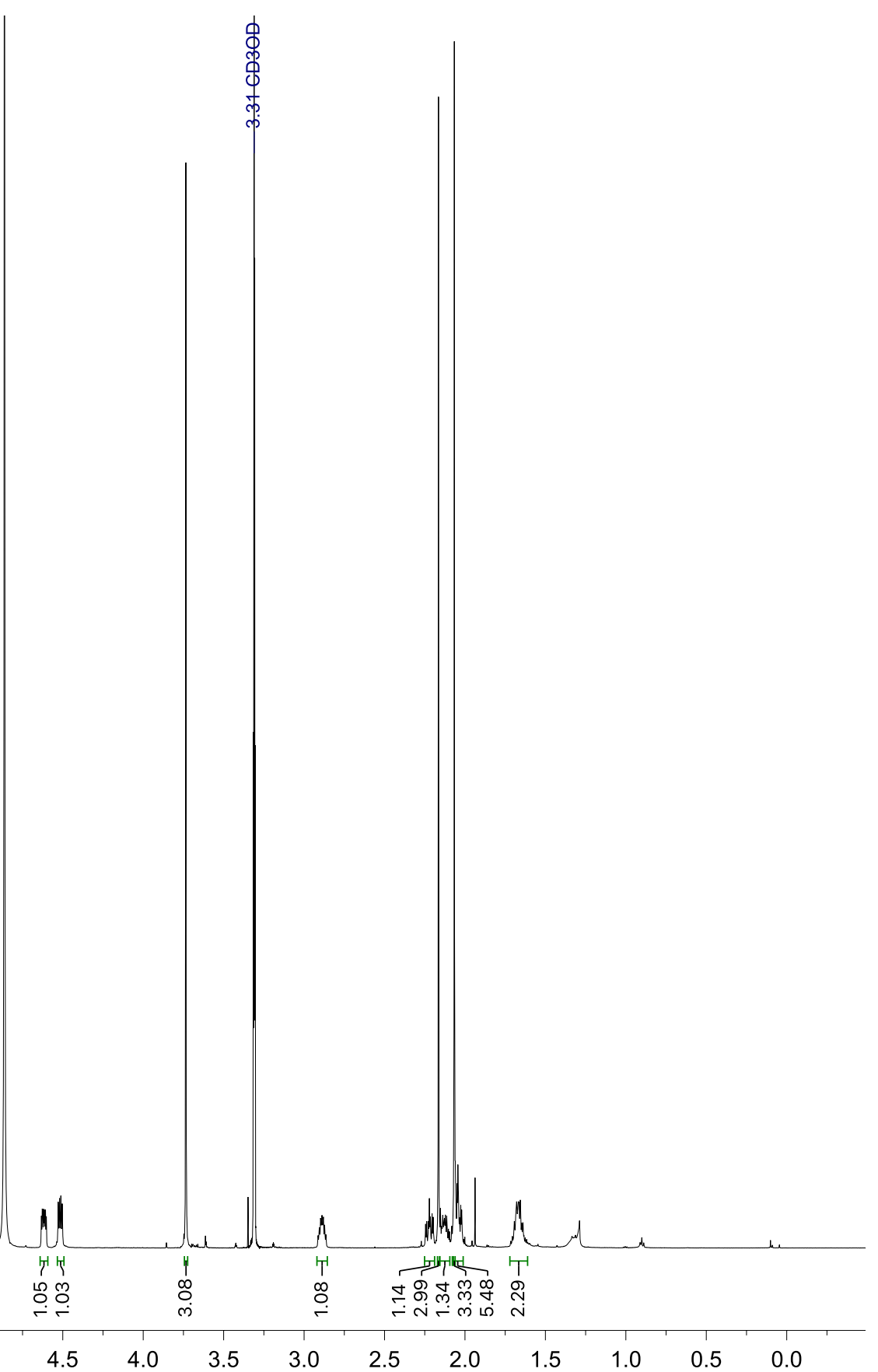




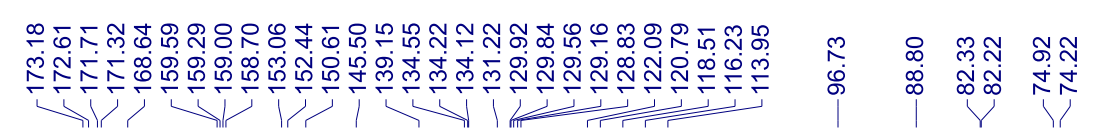

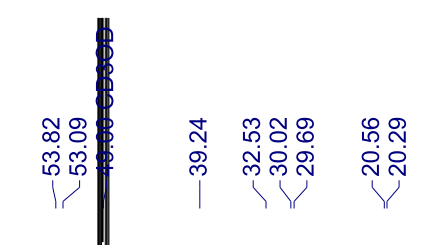

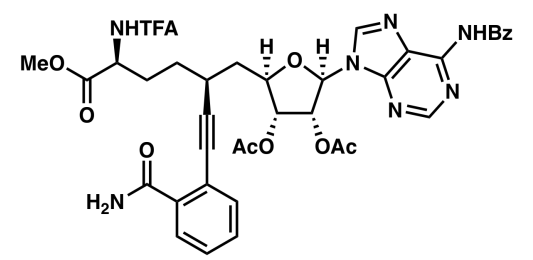

$\mathbf{S 6 8},{ }^{13} \mathrm{C}, 126 \mathrm{MHz}, \mathrm{CD}_{3} \mathrm{OD}$

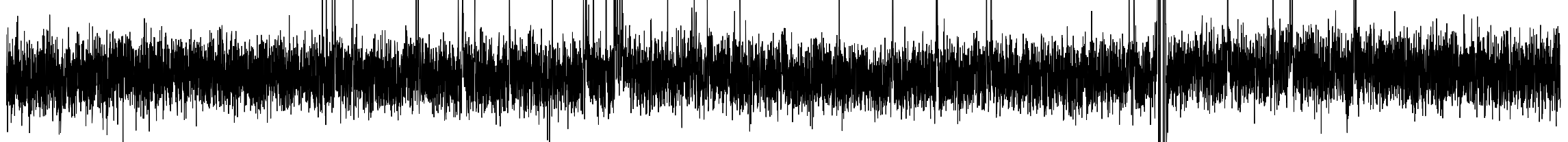

$\begin{array}{llllll}210 & 200 & 190 & 180 & 170 & 160\end{array}$

150

130

120

0100

90

8070

60

$40 \quad 30$

$20 \quad 10$ 0 


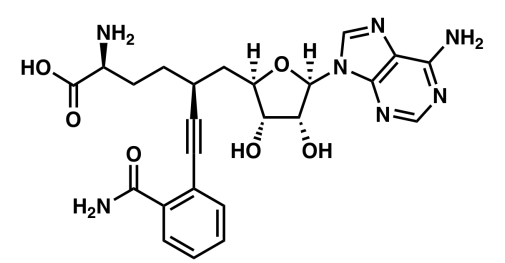

28, ${ }^{1} \mathrm{H}, 600 \mathrm{MHz}, \mathbf{C D}_{3} \mathbf{C N} / \mathrm{D}_{2} \mathrm{O}$

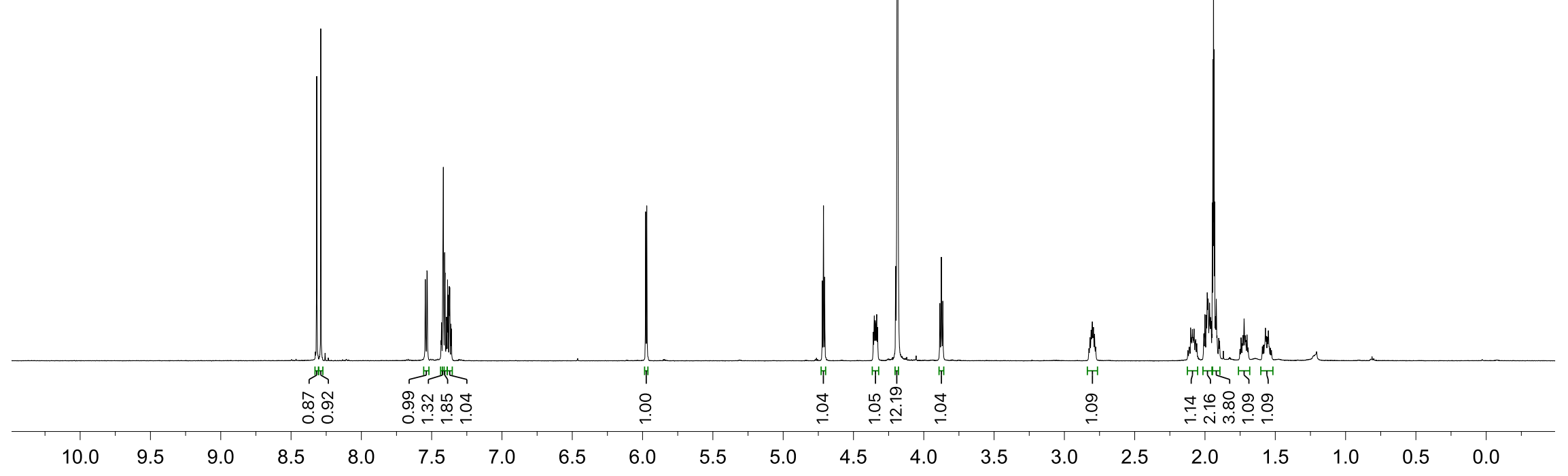




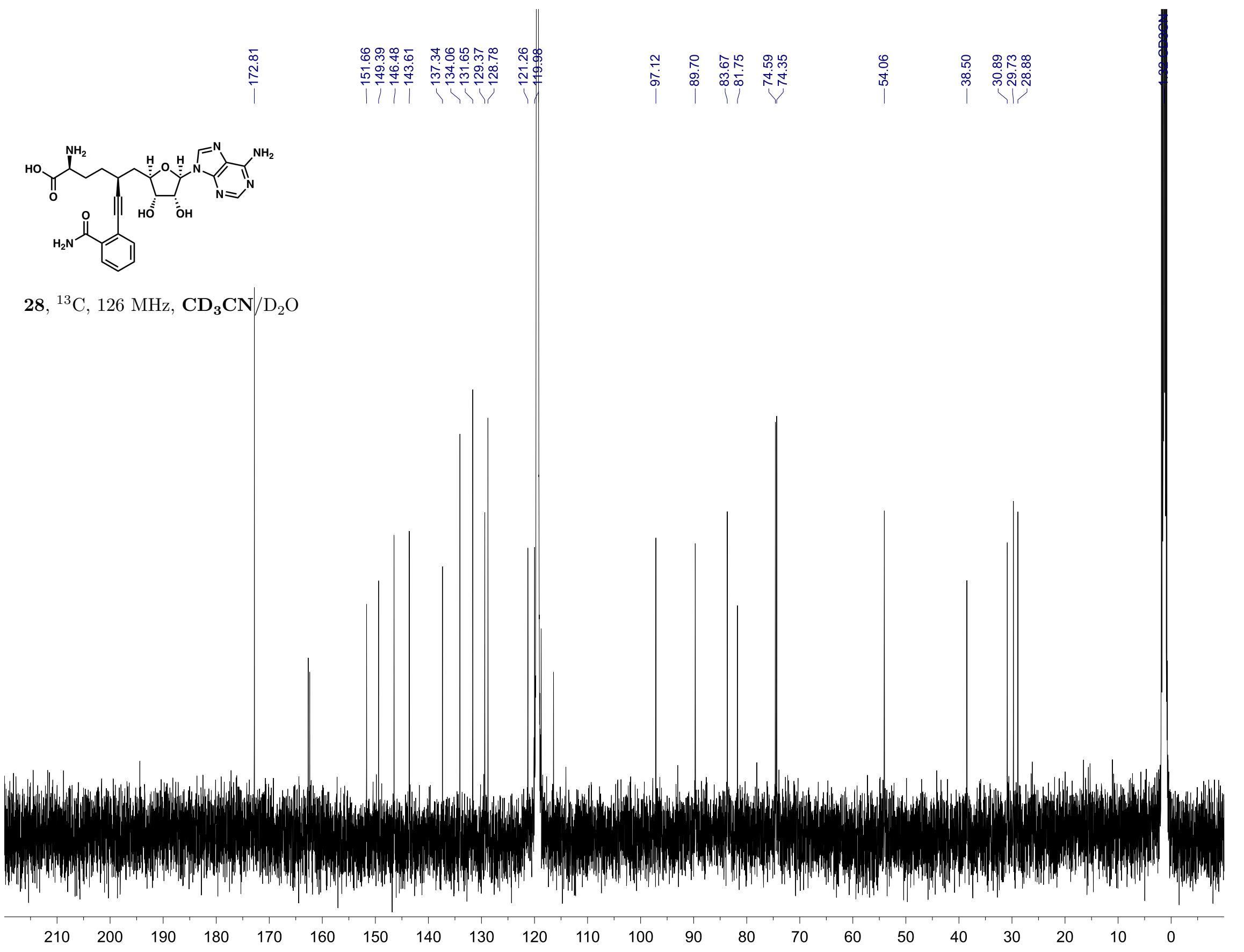




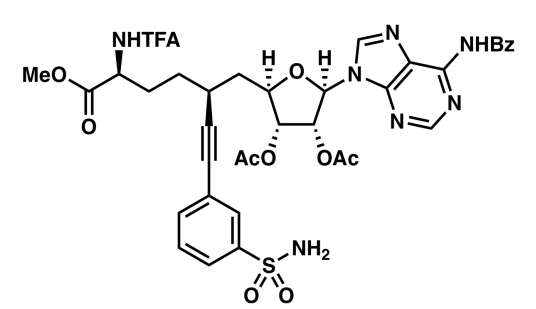

S69, ${ }^{1} \mathrm{H}, 600 \mathrm{MHz}, \mathrm{CDCl}_{3}$ 

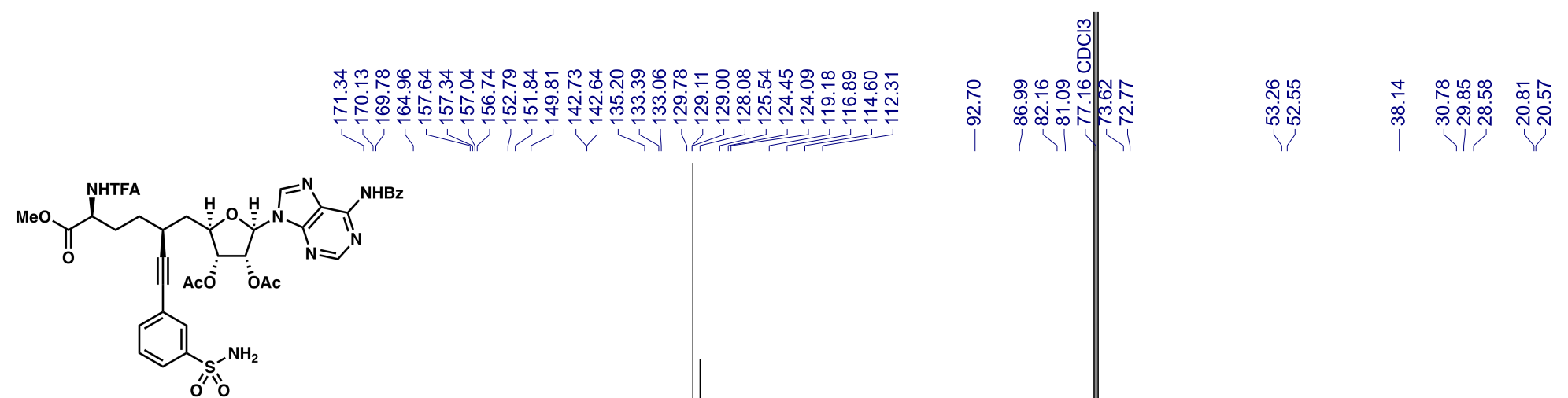

S69, ${ }^{13} \mathrm{C}, 126 \mathrm{MHz}, \mathrm{CDCl}_{3}$

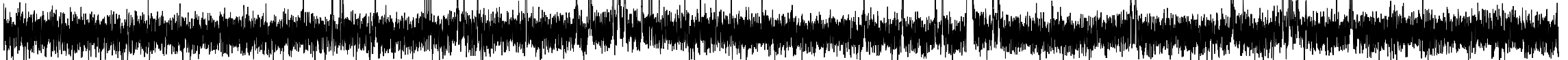




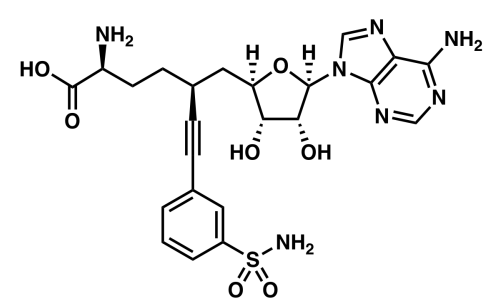

29, ${ }^{1} \mathrm{H}, 600 \mathrm{MHz}, \mathbf{C D}_{\mathbf{3}} \mathbf{C N} / \mathrm{D}_{2} \mathrm{O} / d-\mathrm{TFA}$

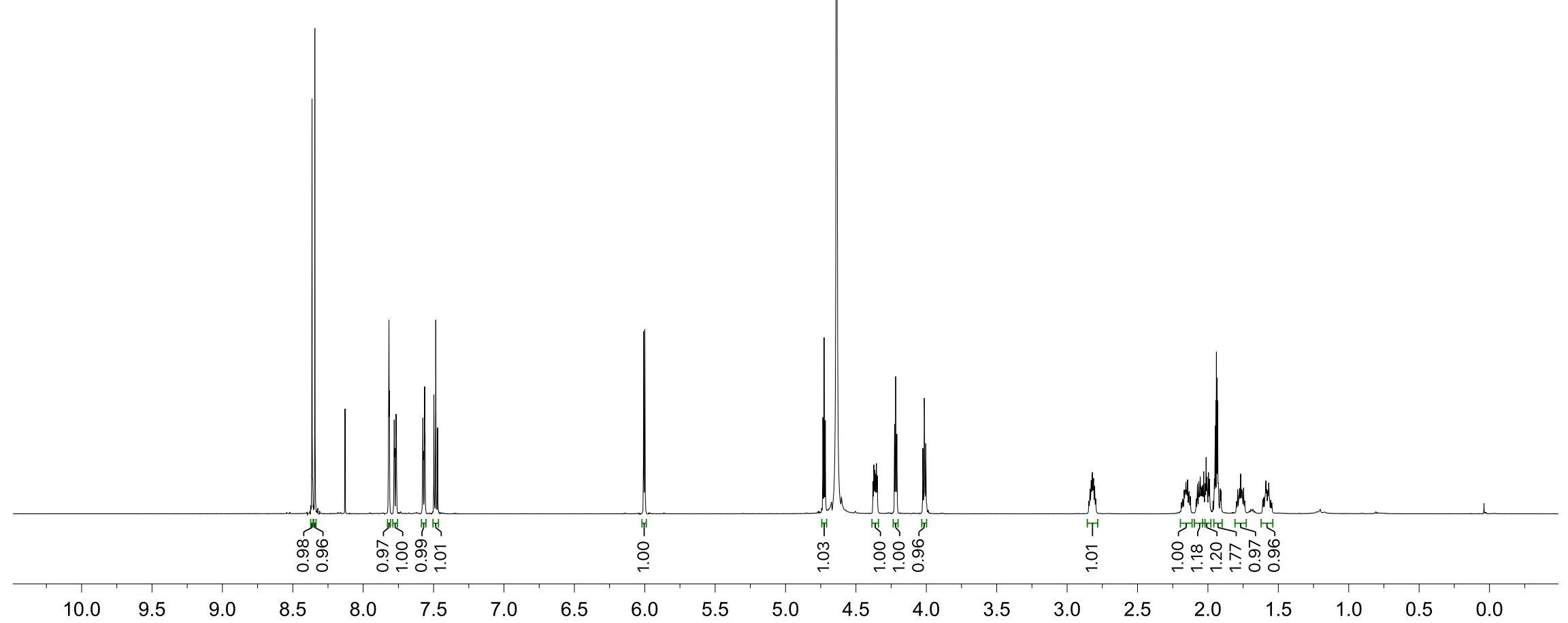




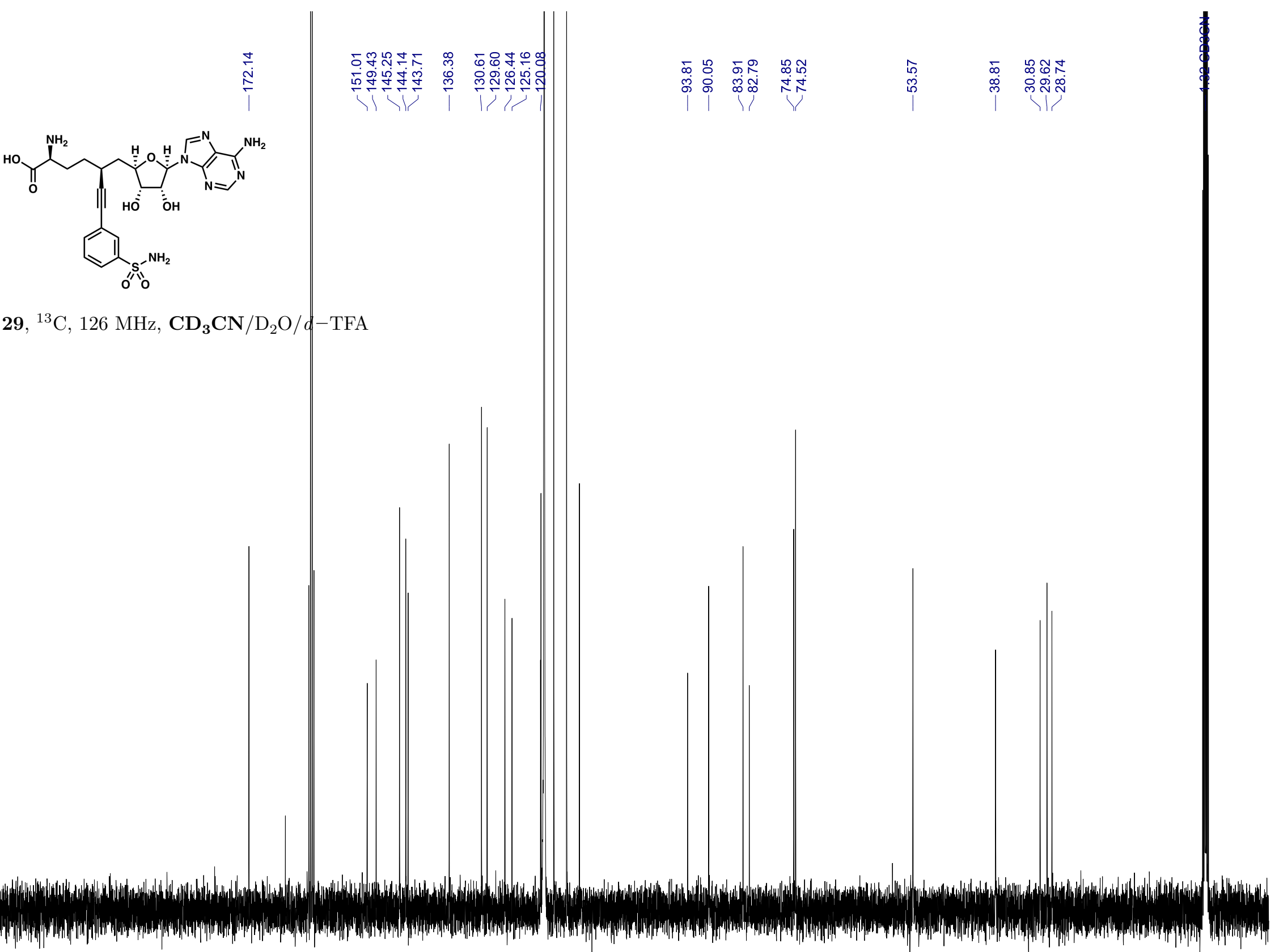

$210 \quad 200 \quad 190$

180

$170 \quad 160$

150

130

$120 \quad 110 \quad 100$

90

$80 \quad 70$

60

50

$40 \quad 30$

$20 \quad 10$

0 


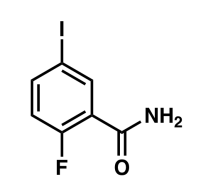

S70, ${ }^{1} \mathrm{H}, 500 \mathrm{MHz}, \mathrm{CD}_{3} \mathrm{OD}$ 


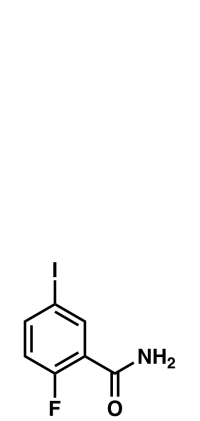

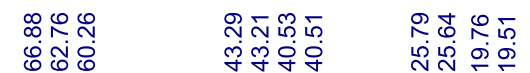

\&

Yo

S70, ${ }^{13} \mathrm{C}, 101 \mathrm{MHz}, \mathrm{CD}_{3} \mathrm{OD}$

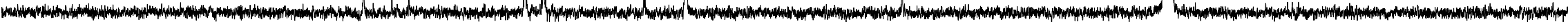




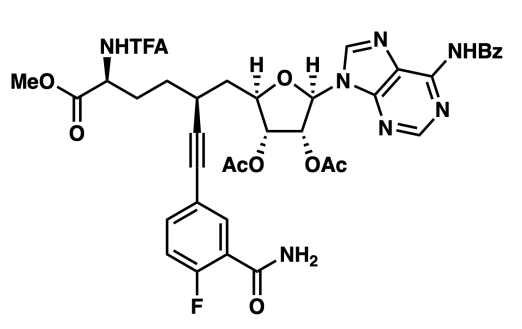

$\mathrm{S} 71,{ }^{1} \mathrm{H}, 600 \mathrm{MHz}, \mathrm{CDCl}_{3}$

$\stackrel{\Xi}{\bullet}$

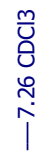

i̊

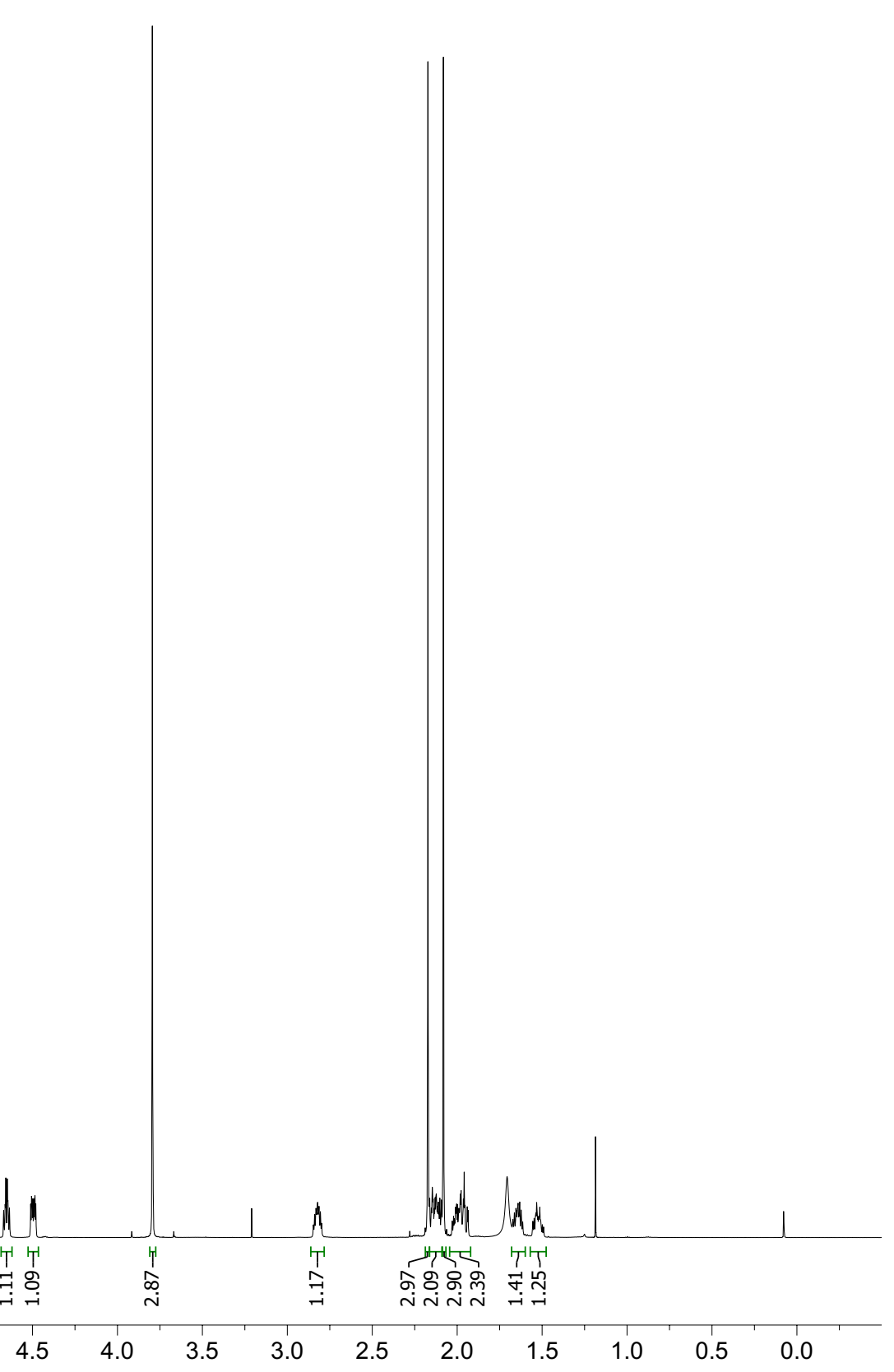




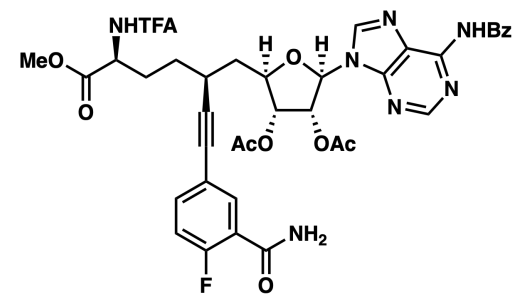

$\mathbf{S 7 1},{ }^{13} \mathrm{C}, 101 \mathrm{MHz}, \mathrm{CDCl}_{3}$

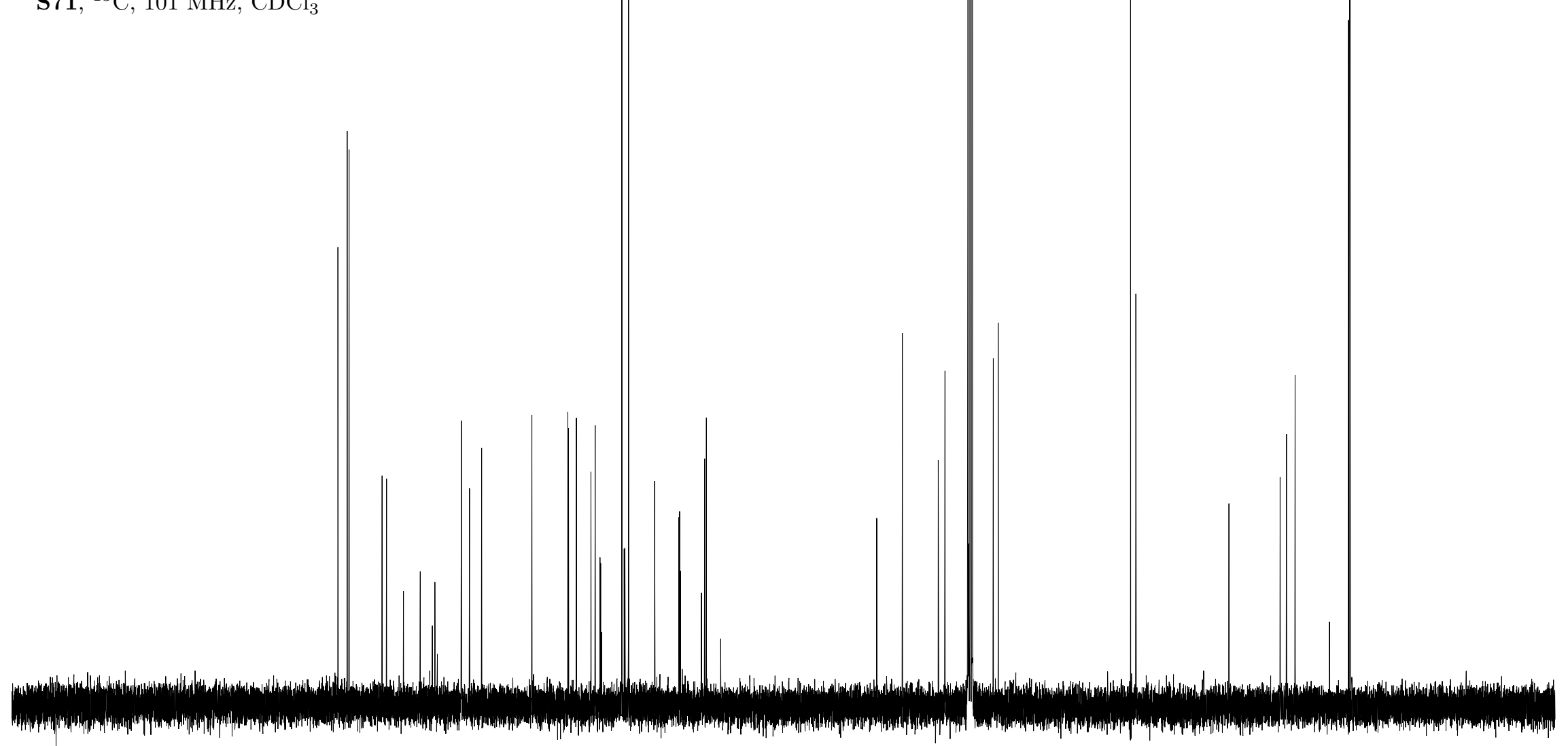




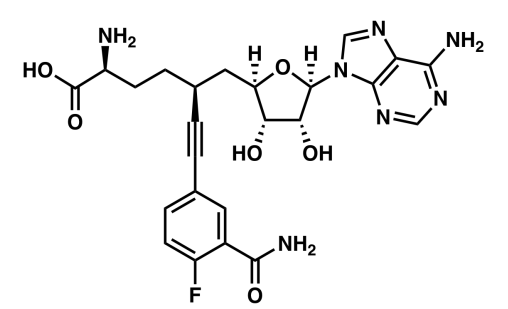

30, ${ }^{1} \mathrm{H}, 600 \mathrm{MHz}, \mathbf{C D}_{\mathbf{3}} \mathbf{C N} / \mathrm{D}_{2} \mathrm{O} / d-\mathrm{TFA}$

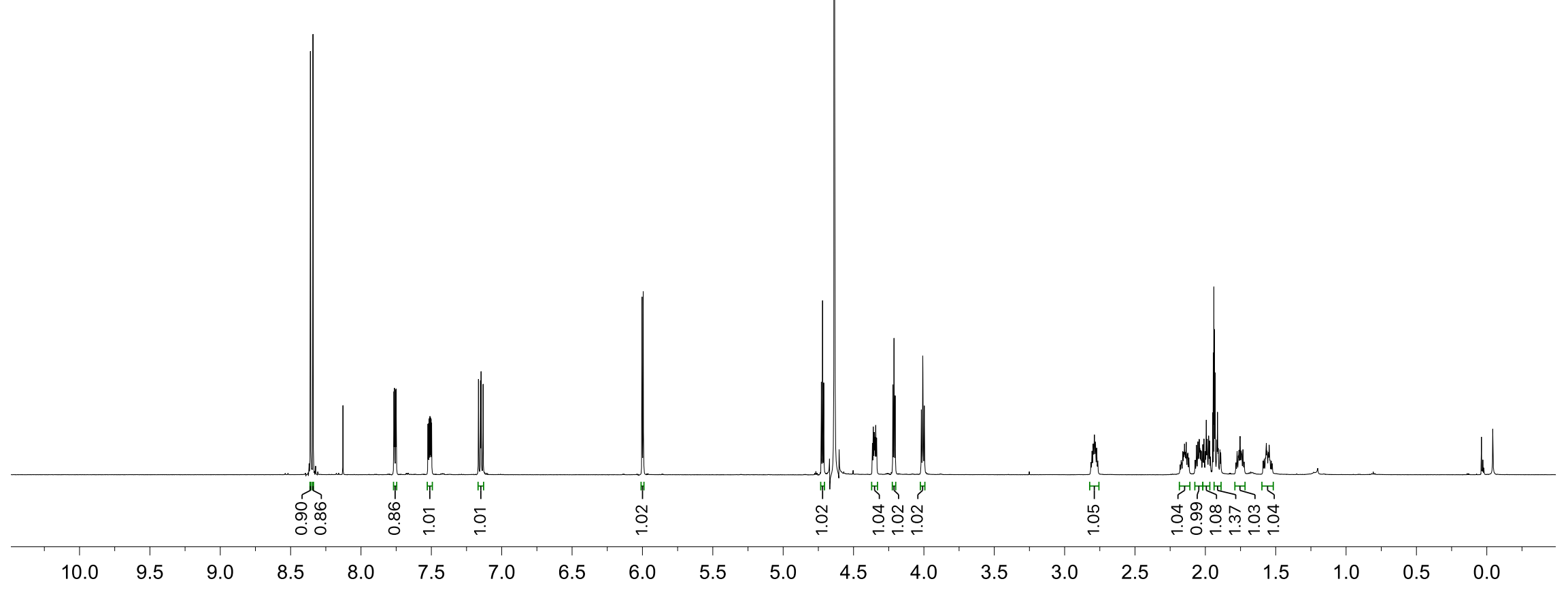




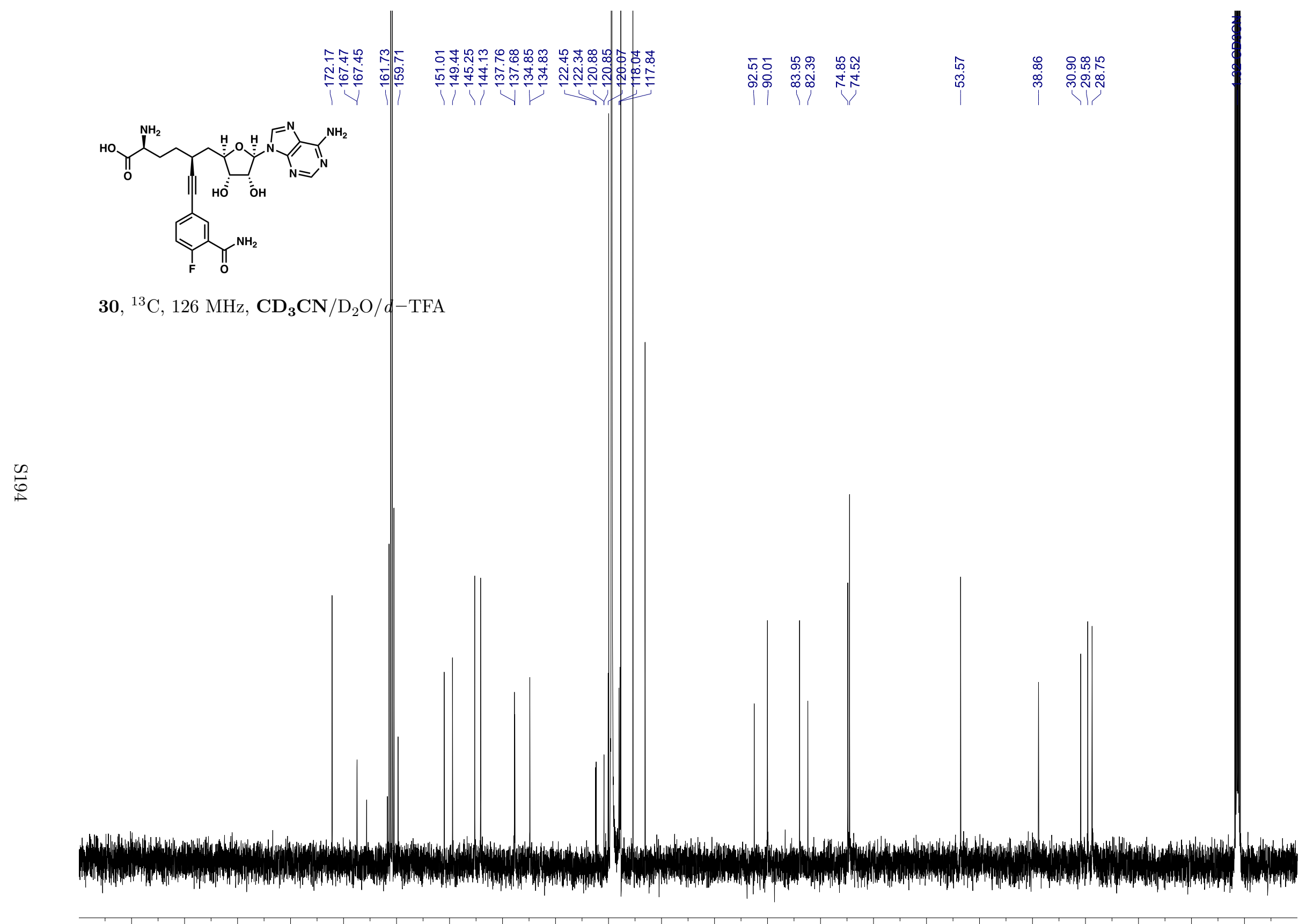

190

180

$170 \quad 160$

150

130

$110 \quad 100$

90

$80 \quad 70$

$60 \quad 50$

$40 \quad 30$

$20 \quad 10$

0 


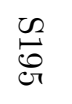

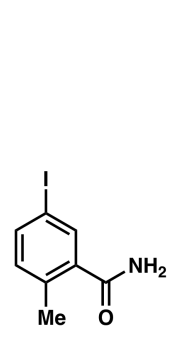

$\mathbf{S 7 2},{ }^{1} \mathrm{H}, 600 \mathrm{MHz}, \mathrm{CDCl}_{3}$

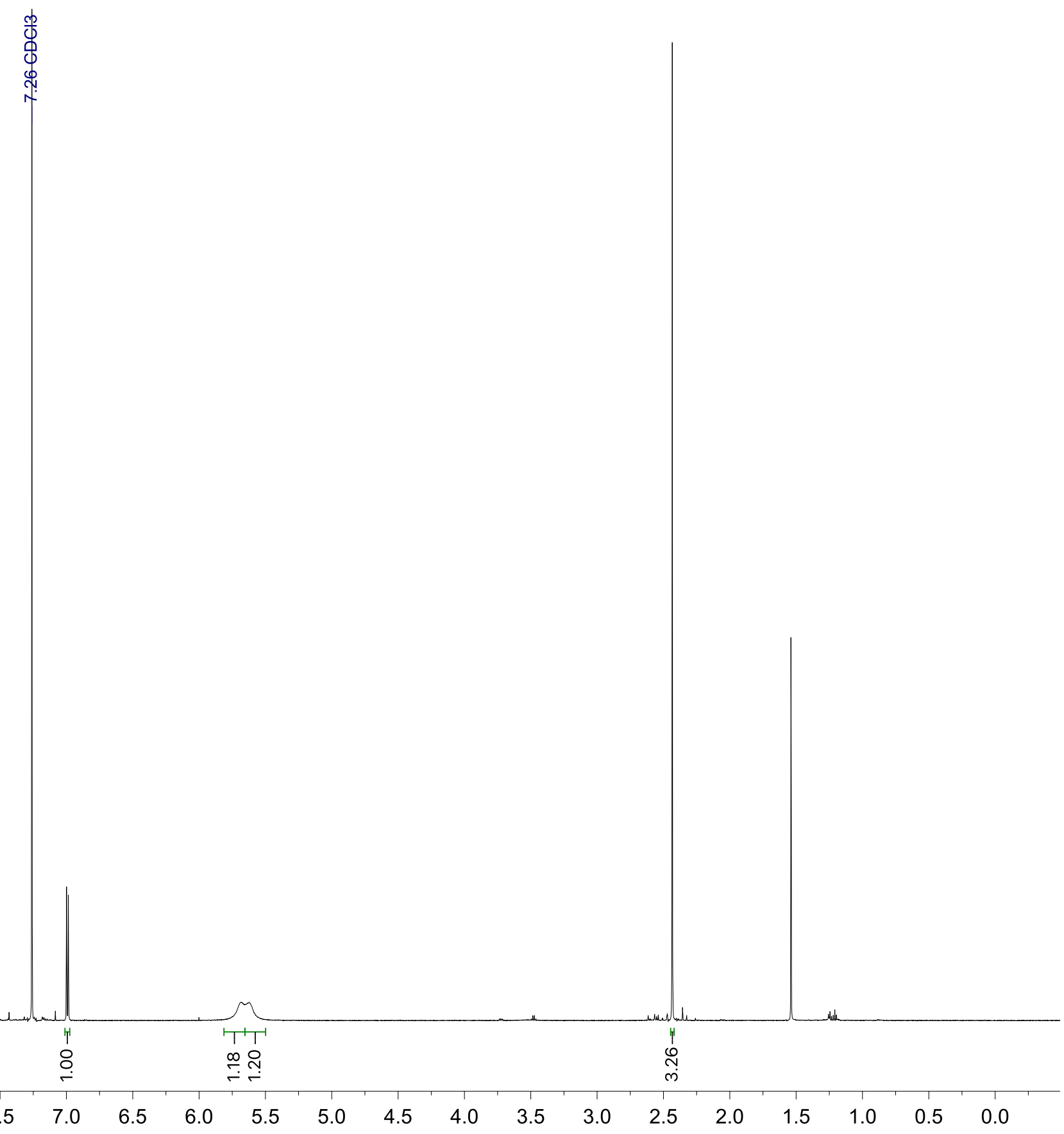




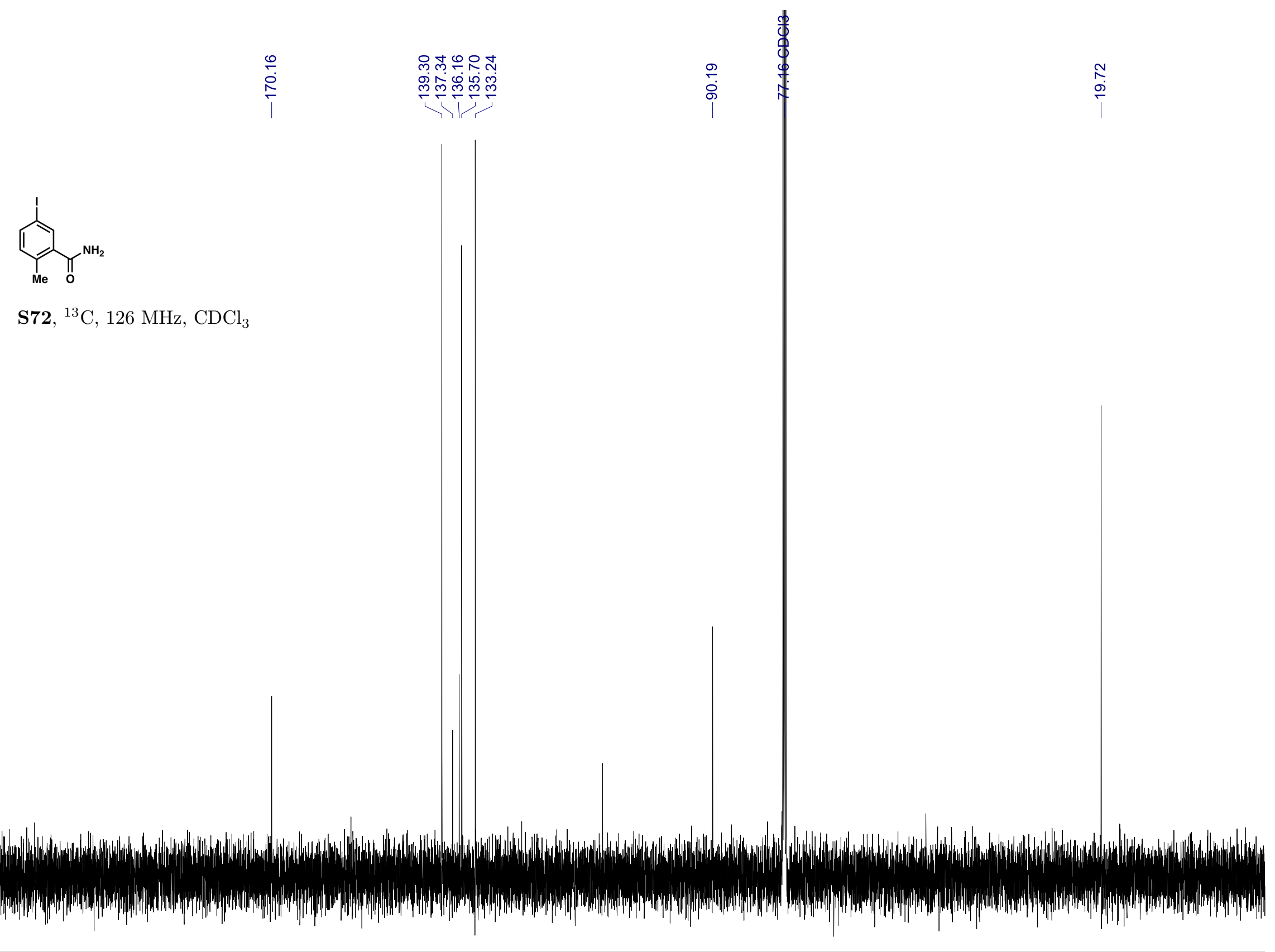

150

130

90

6050 


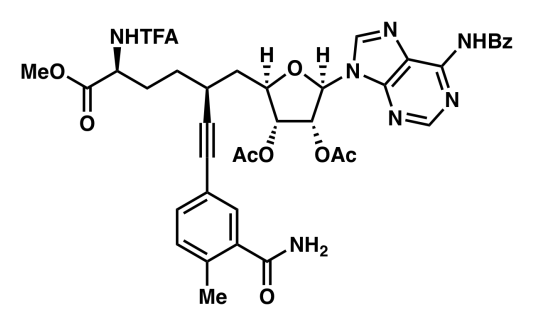

S73, ${ }^{1} \mathrm{H}, 500 \mathrm{MHz}, \mathrm{CDCl}_{3}$

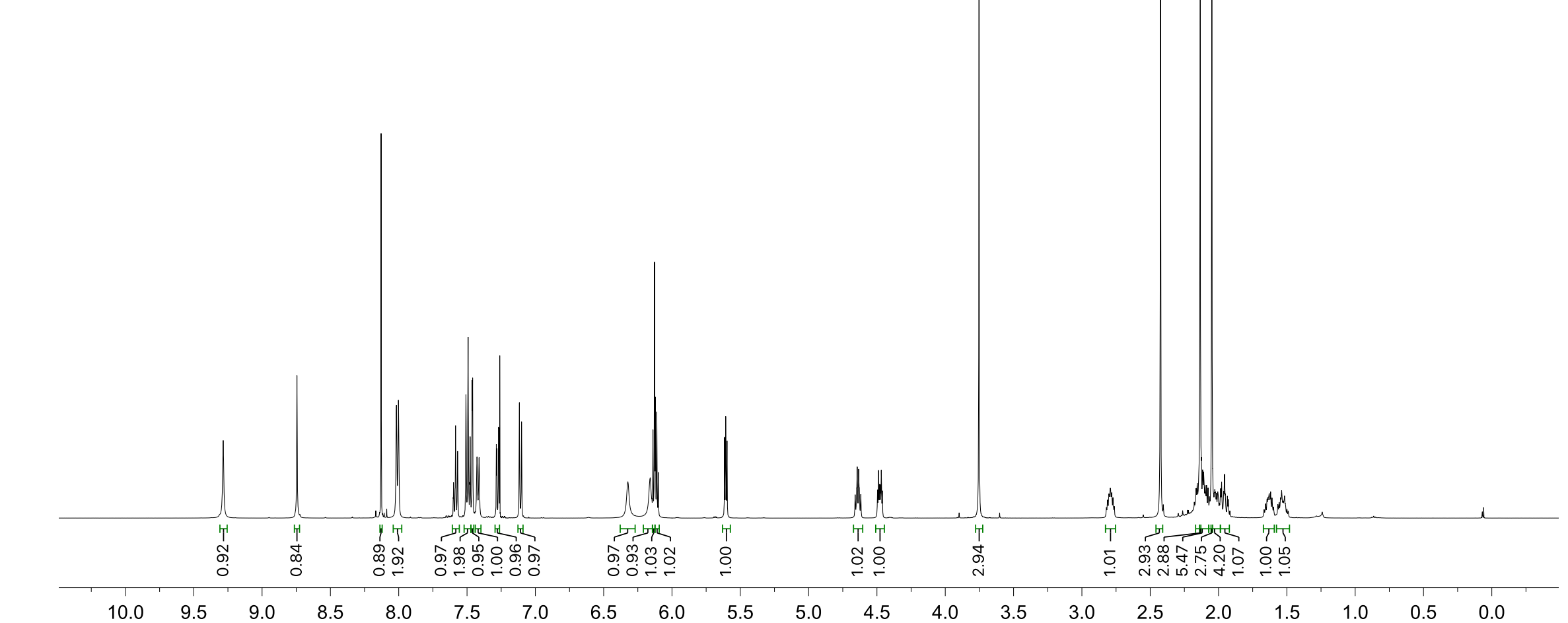




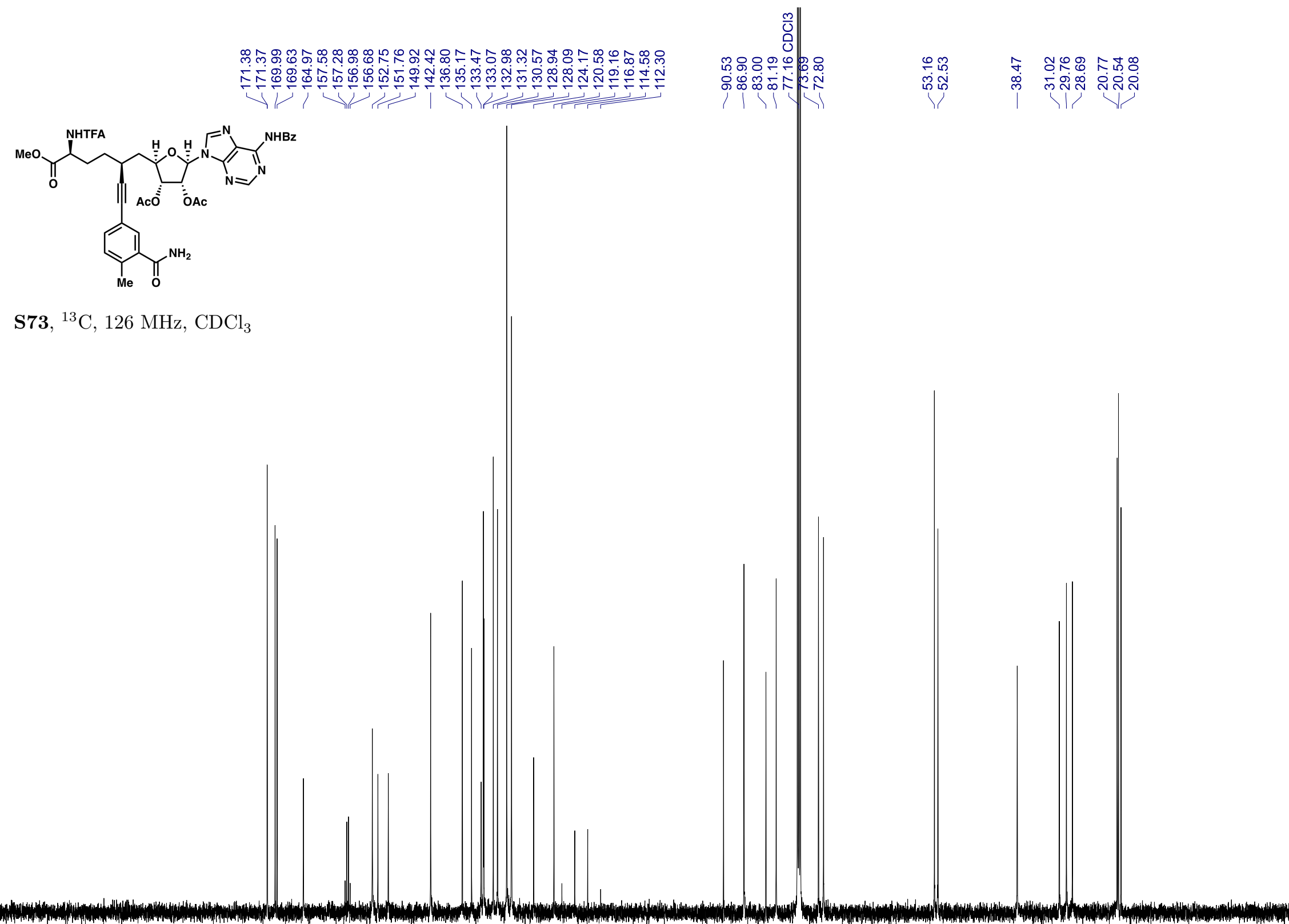

$210 \quad 200$

190

180

$170 \quad 160$

150

140

130

120

110

100

90

80

70

60

50

$40 \quad 30$

$20 \quad 10$ 


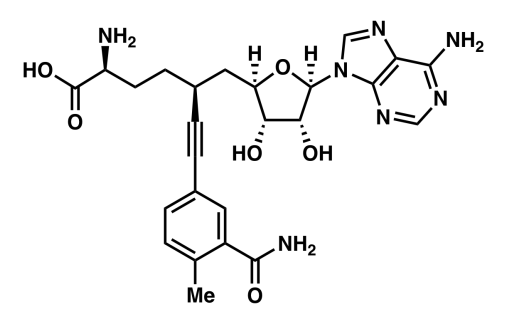

31, ${ }^{1} \mathrm{H}, 600 \mathrm{MHz}, \mathbf{C D}_{\mathbf{3}} \mathbf{C N} / \mathrm{D}_{2} \mathrm{O} / d-\mathrm{TFA}$
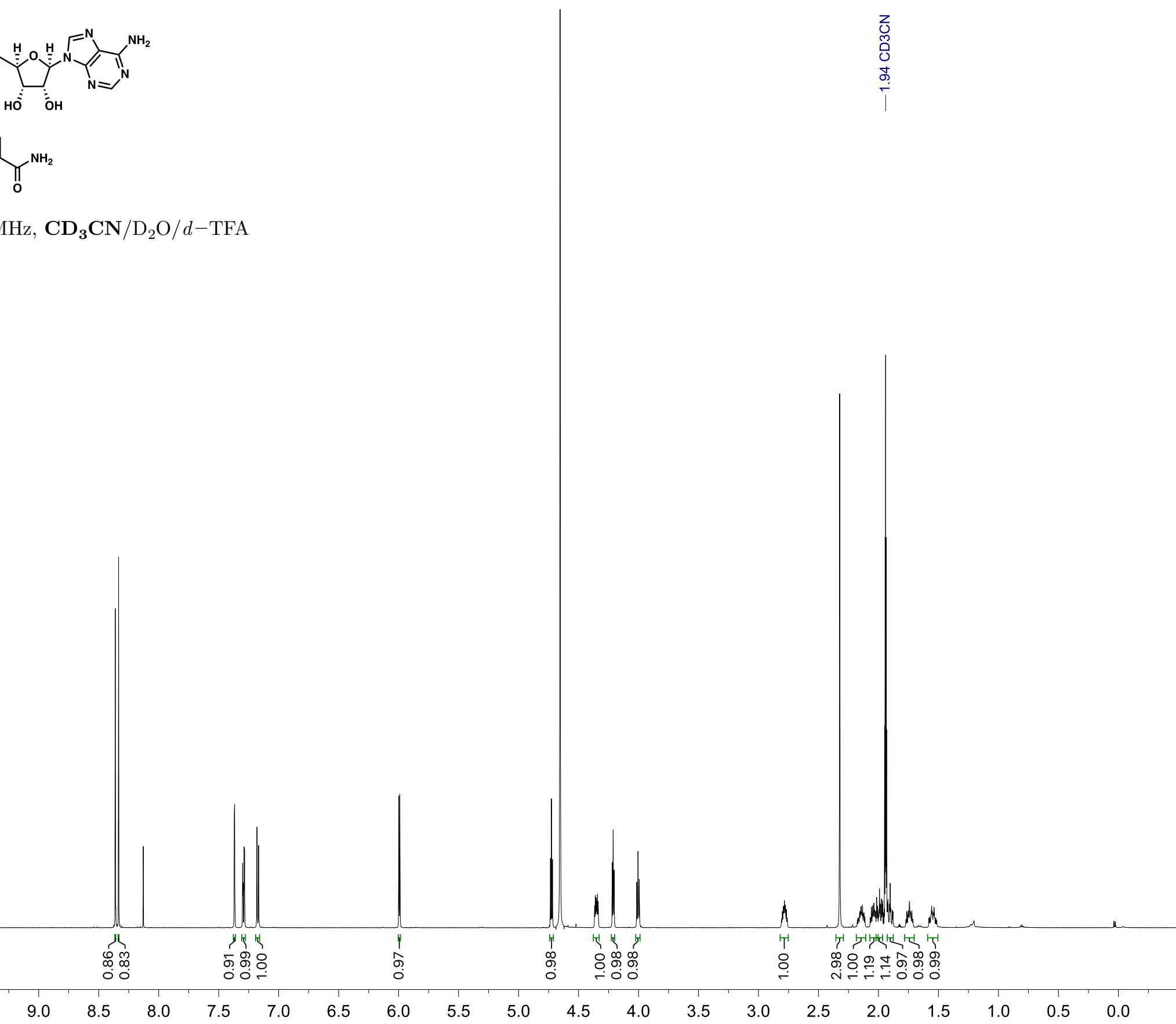


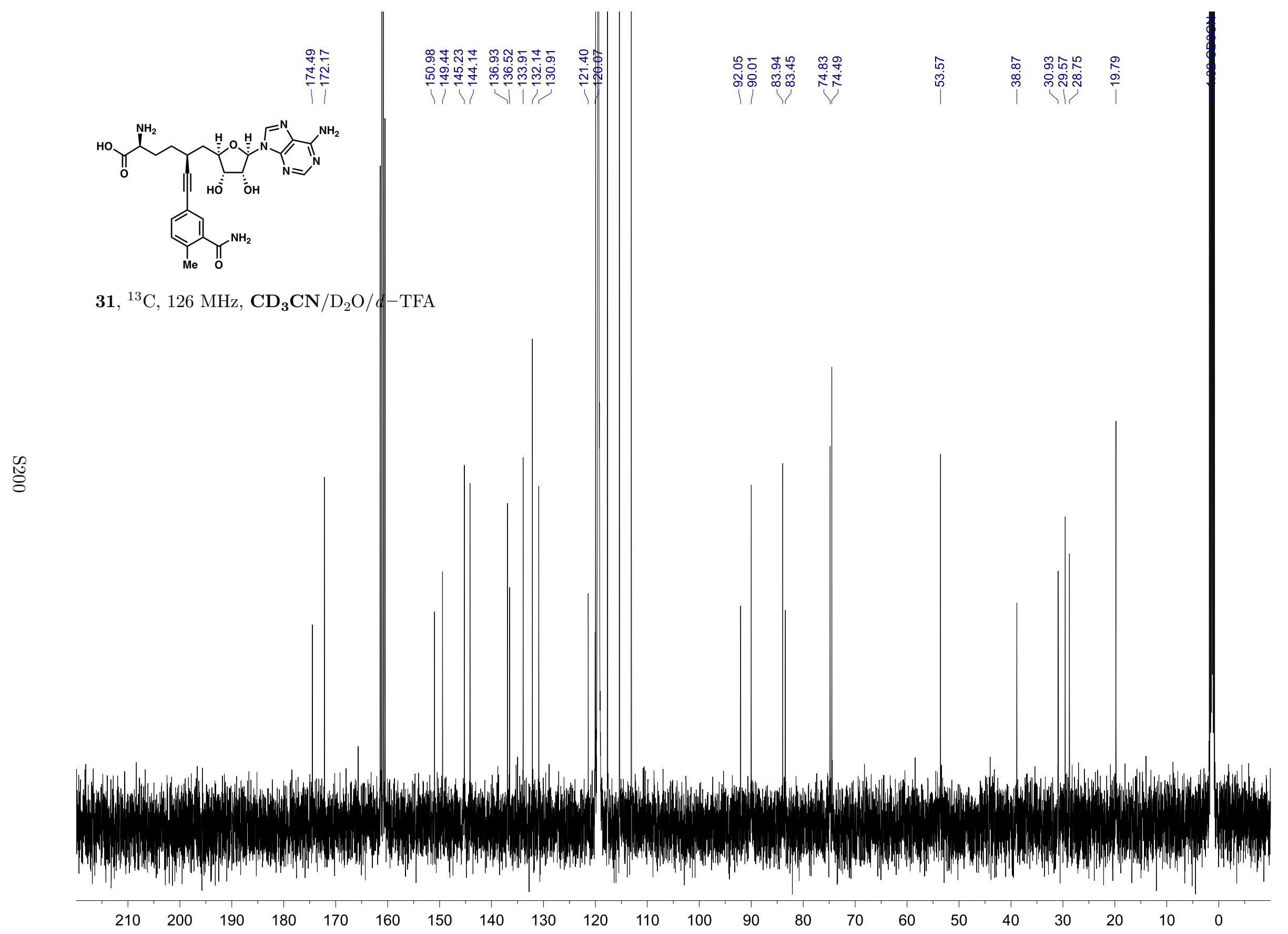




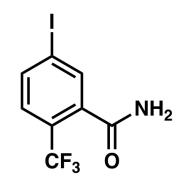

$\mathbf{S 7 4},{ }^{1} \mathrm{H}, 500 \mathrm{MHz}, \mathrm{CD}_{3} \mathrm{OD}$

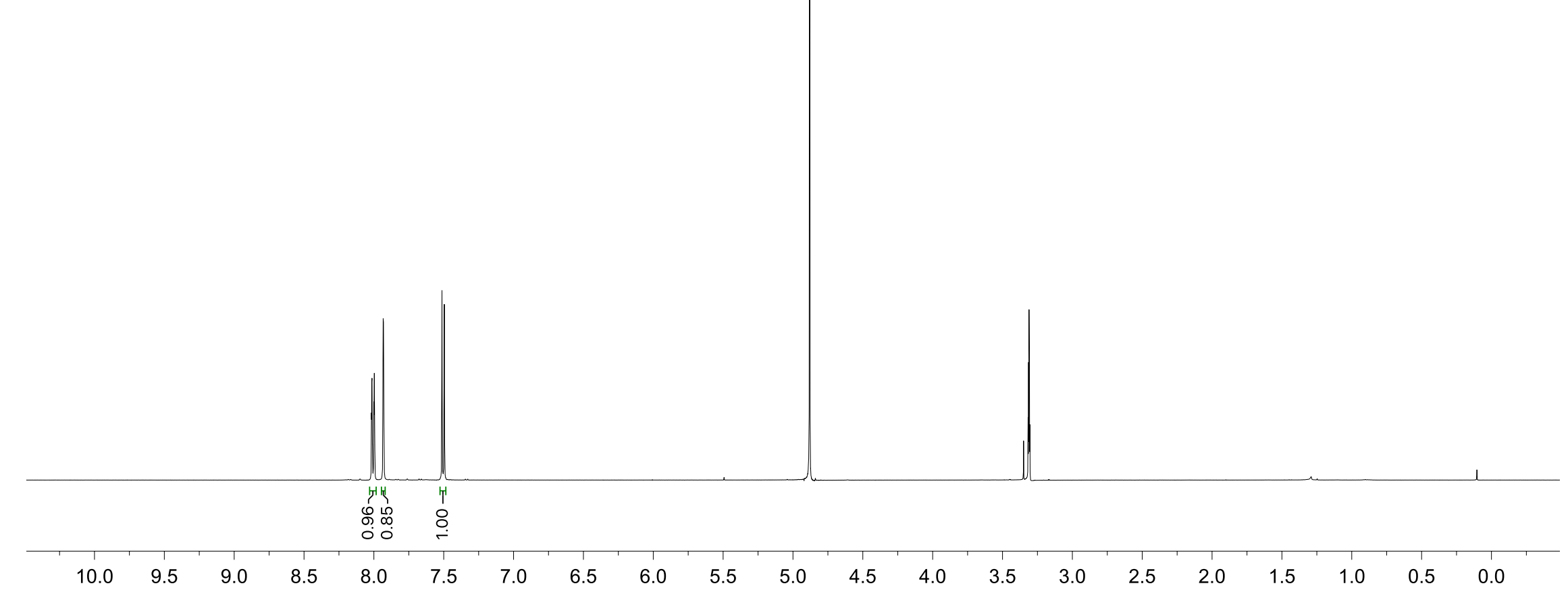




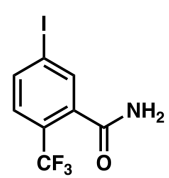

S74, ${ }^{13} \mathrm{C}, 126 \mathrm{MHz}, \mathrm{CD}_{3} \mathrm{OD}$

柋

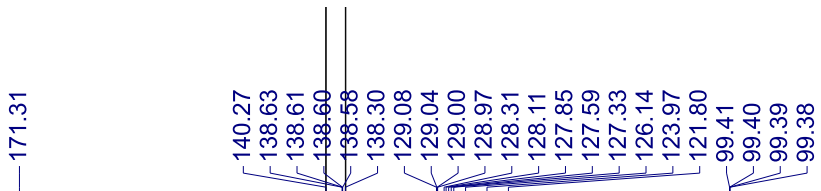

m̊ 


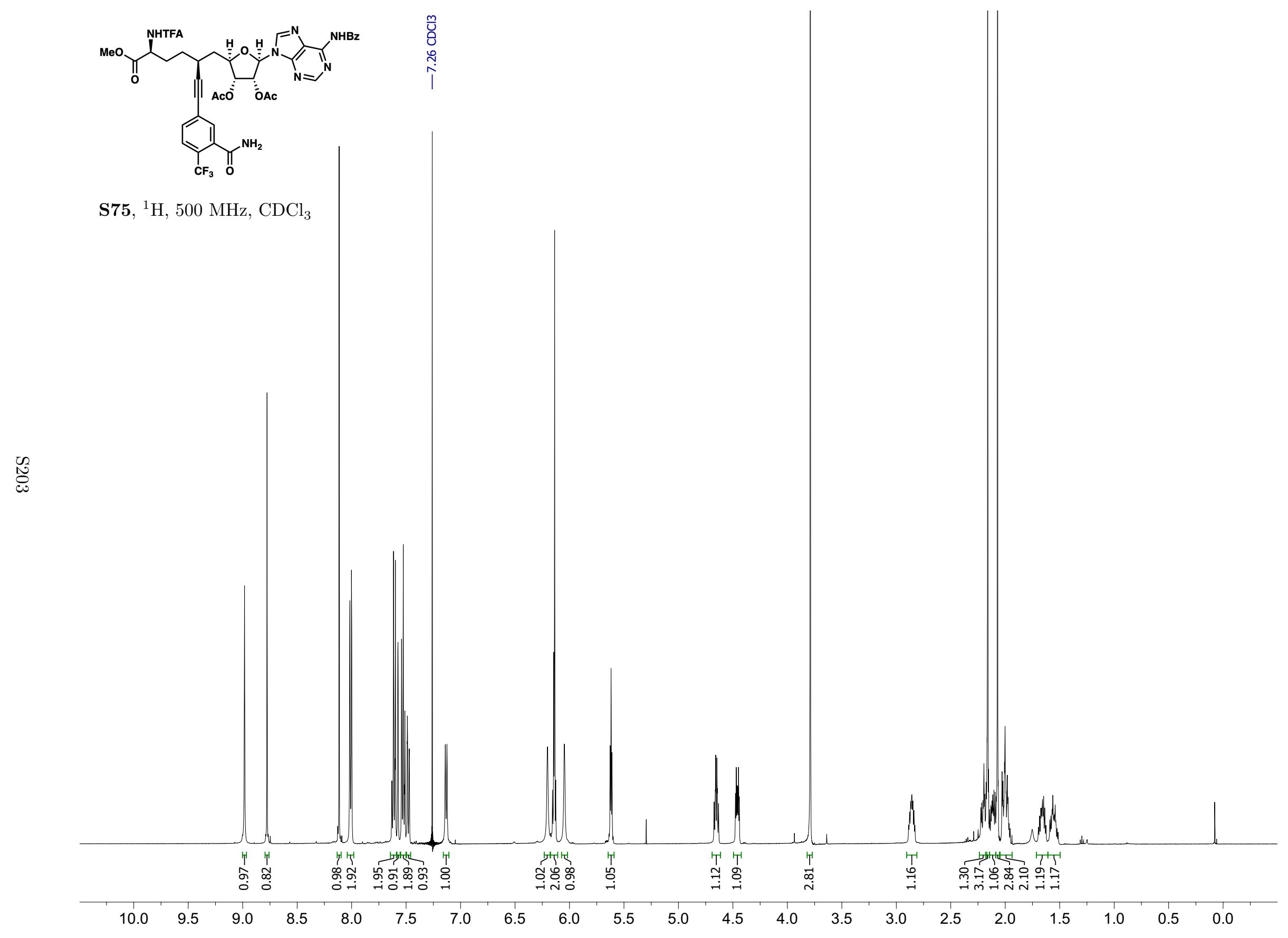




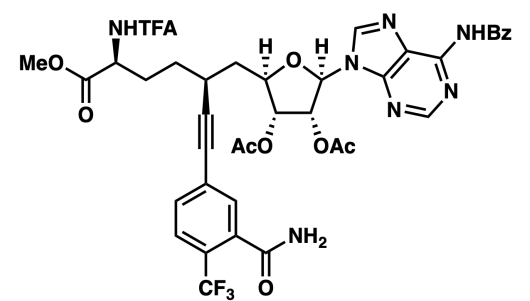

S75, ${ }^{13} \mathrm{C}, 101 \mathrm{MHz}, \mathrm{CDCl}_{3}$

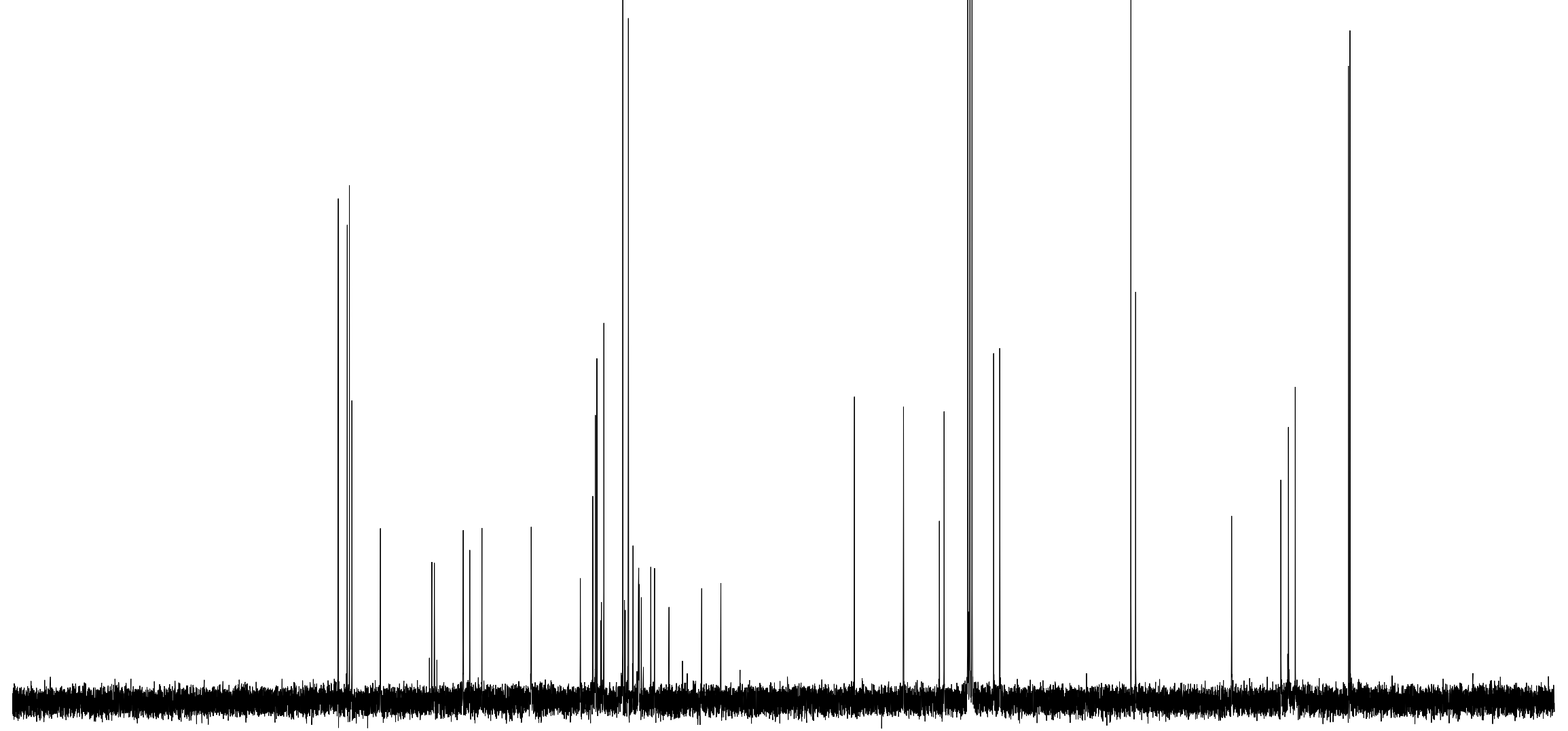




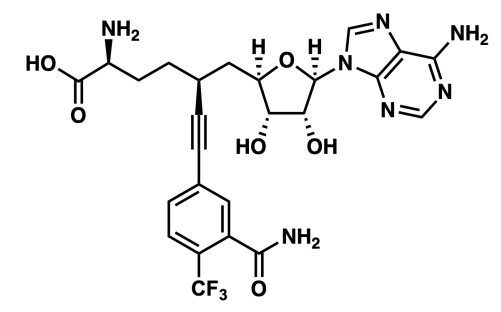

32, ${ }^{1} \mathrm{H}, 600 \mathrm{MHz}, \mathrm{CD}_{3} \mathrm{CN} / \mathbf{D}_{\mathbf{2}} \mathbf{O} / d-\mathrm{TFA}$

No

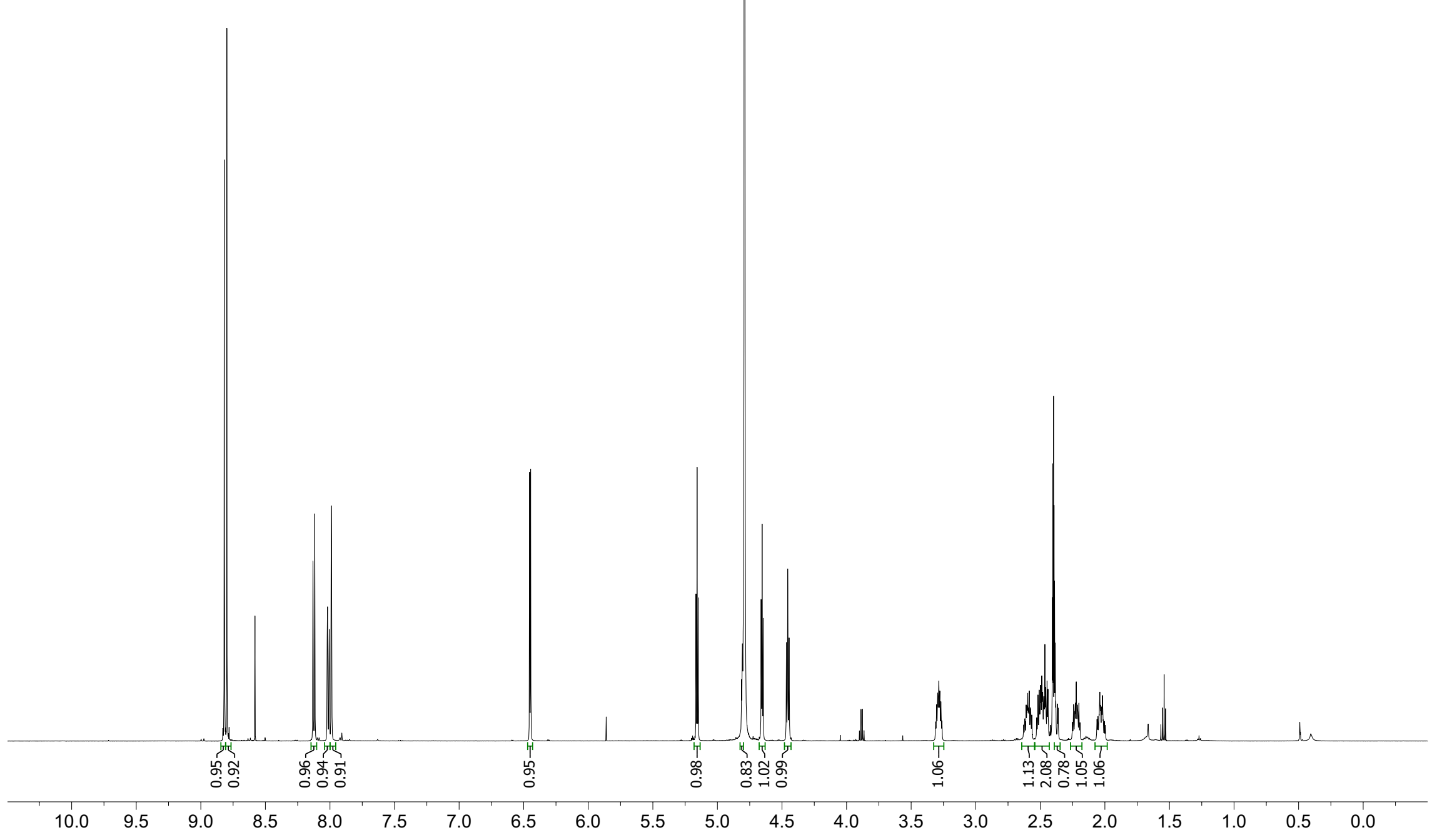



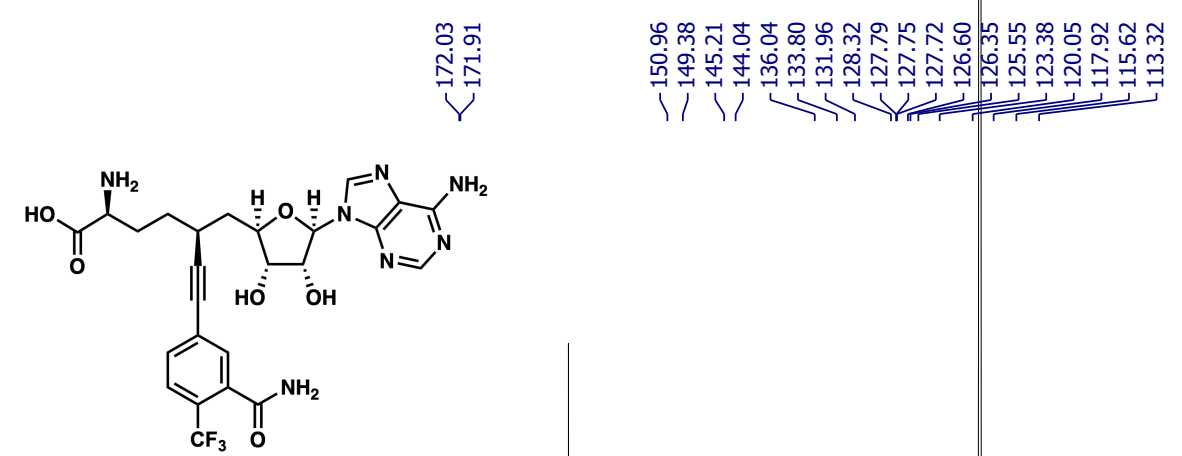

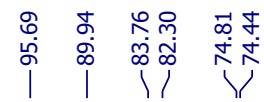

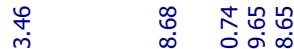

V

32, ${ }^{13} \mathrm{C}, 126 \mathrm{MHz}, \mathbf{C D}_{\mathbf{3}} \mathbf{C N} / \mathrm{D}_{2} \mathrm{O} / d-\mathrm{TFA}$

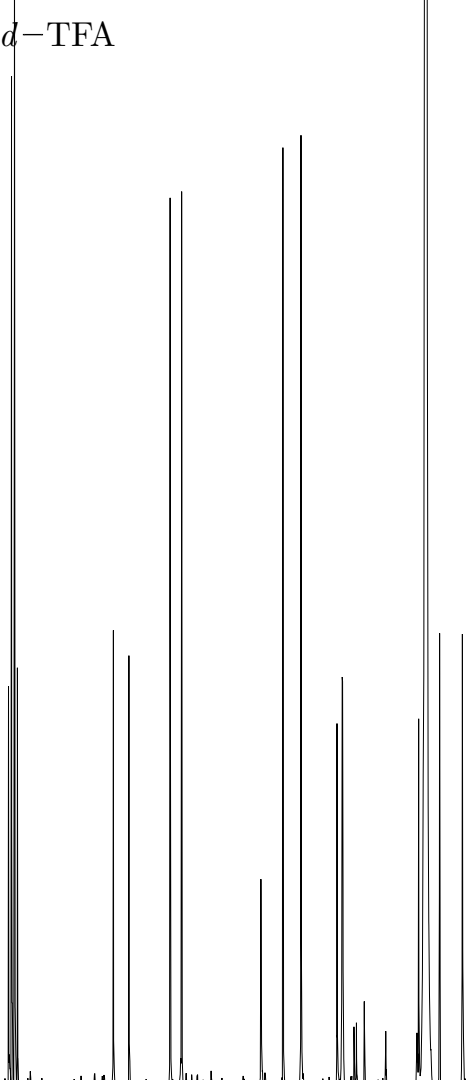

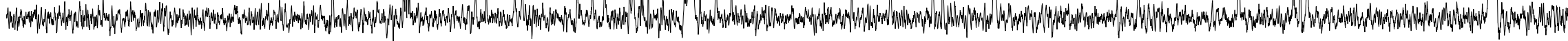

$\begin{array}{llll}190 & 180 & 170 & 160\end{array}$

$150 \quad 140 \quad 130$

$110 \quad 100 \quad 90$

$80 \quad 70$

60

50

$40 \quad 30$

20 


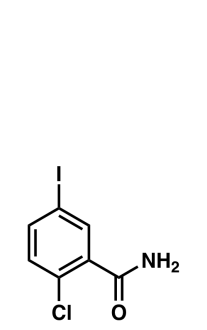

S76, ${ }^{1} \mathrm{H}, 500 \mathrm{MHz}, \mathrm{CD}_{3} \mathrm{OD}$

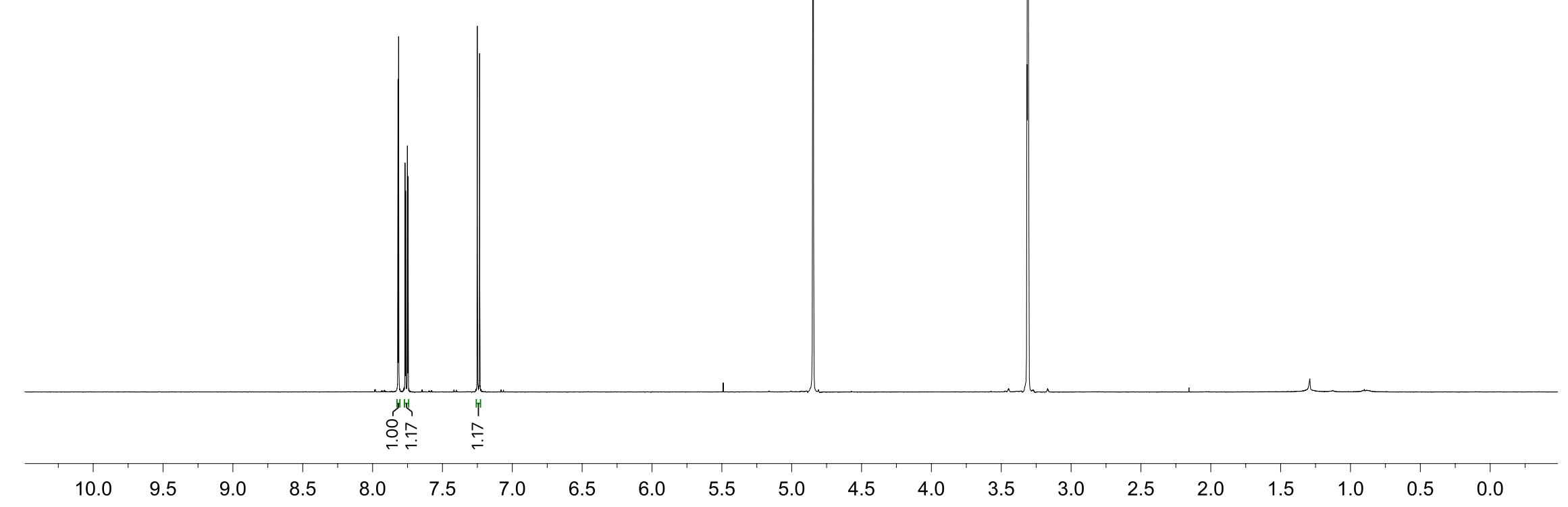




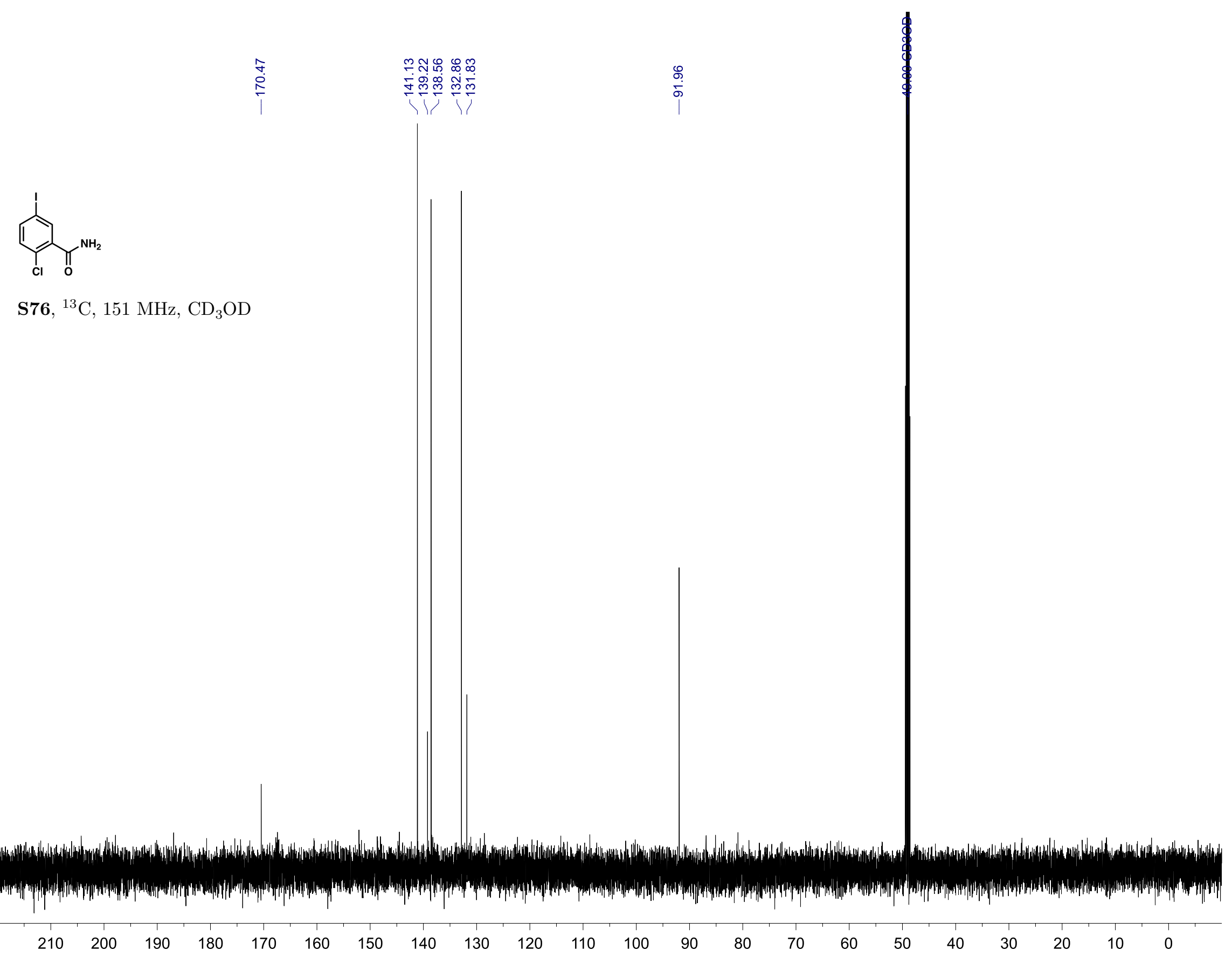




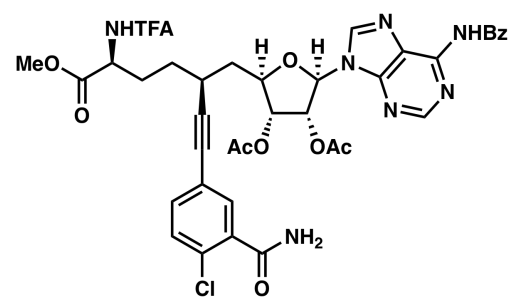

$\mathbf{S 7 7},{ }^{1} \mathrm{H}, 600 \mathrm{MHz}, \mathrm{CDCl}_{3}$

용

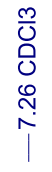

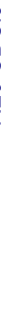




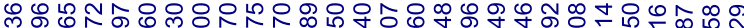

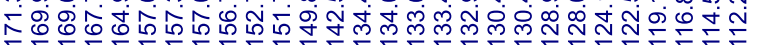

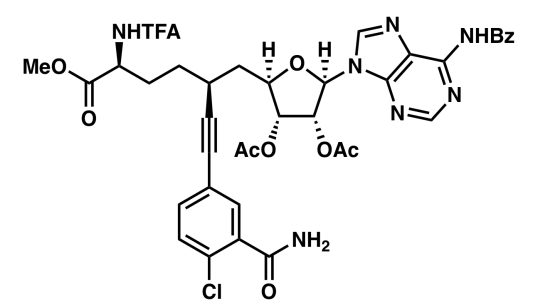

S77, ${ }^{13} \mathrm{C}, 126 \mathrm{MHz}, \mathrm{CDCl}_{3}$ 


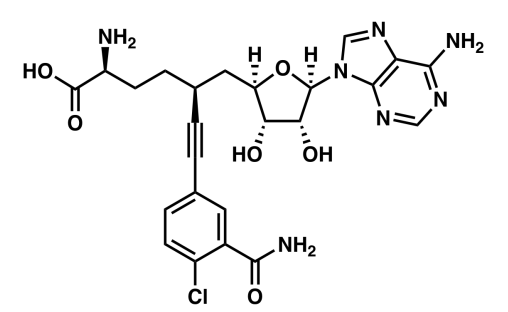

33, ${ }^{1} \mathrm{H}, 600 \mathrm{MHz}, \mathbf{C D}_{\mathbf{3}} \mathbf{C N} / \mathrm{D}_{2} \mathrm{O} / d-\mathrm{TFA}$

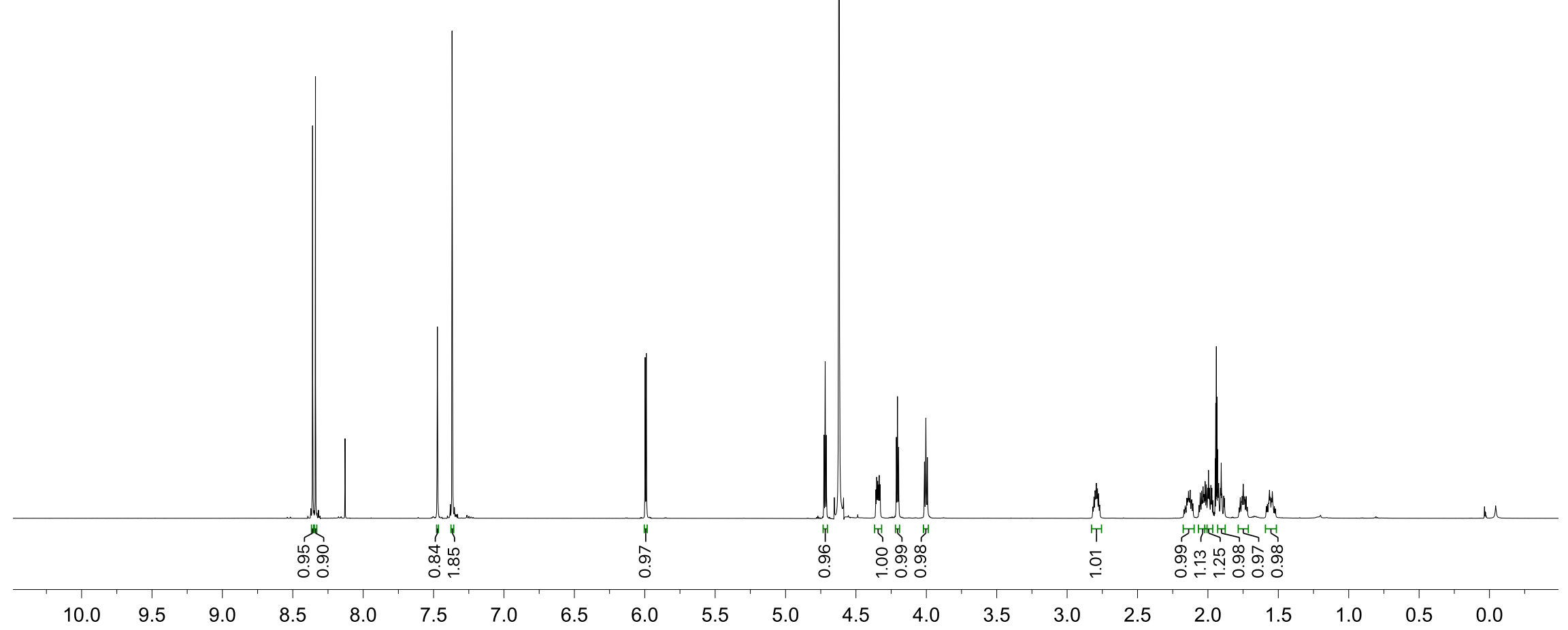




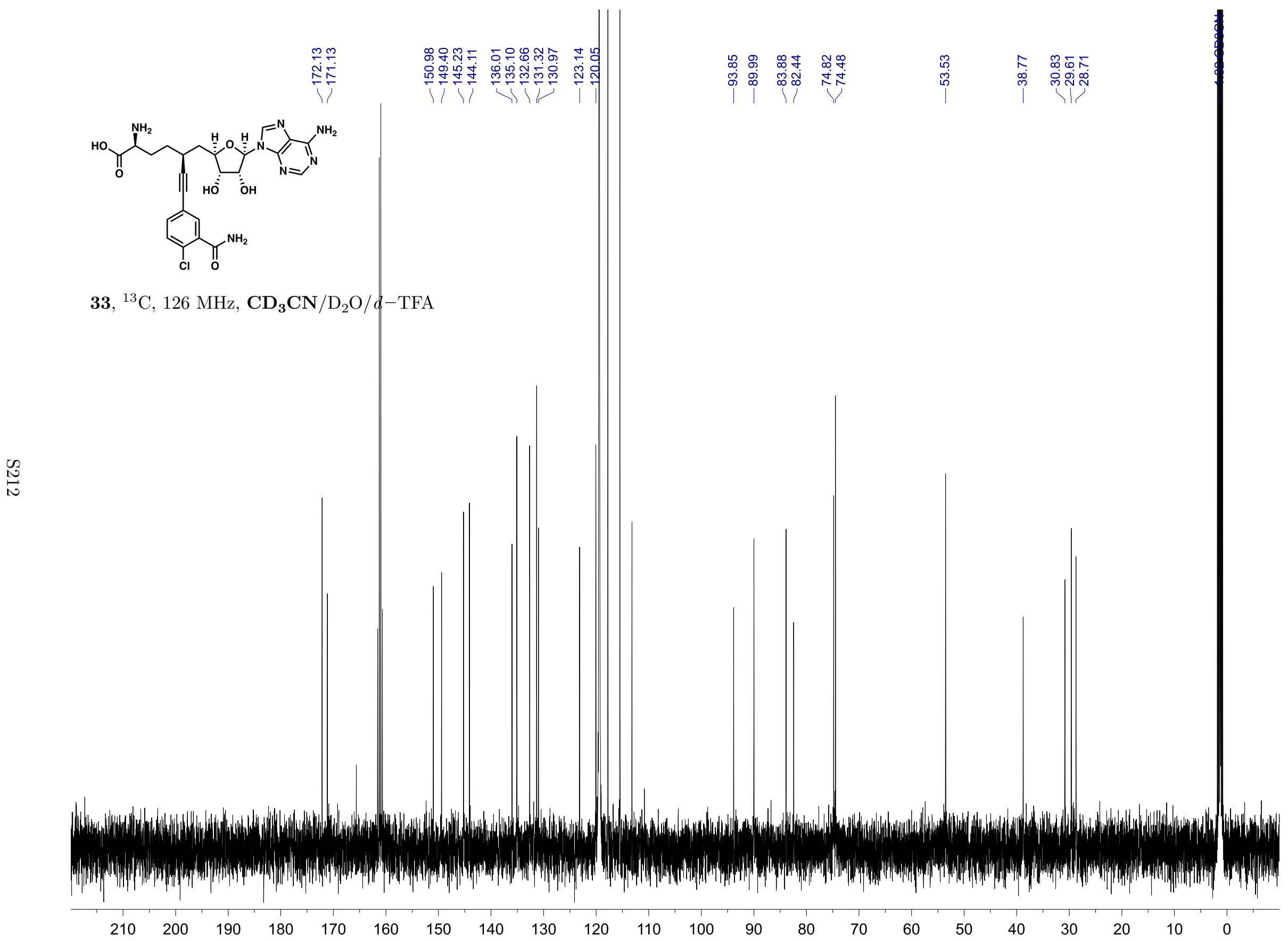




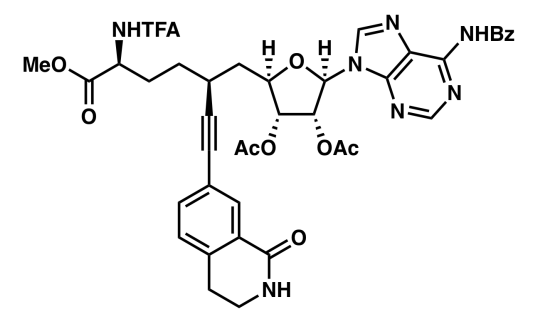

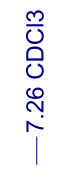

S78, ${ }^{1} \mathrm{H}, 600 \mathrm{MHz}, \mathrm{CDCl}_{3}$ 


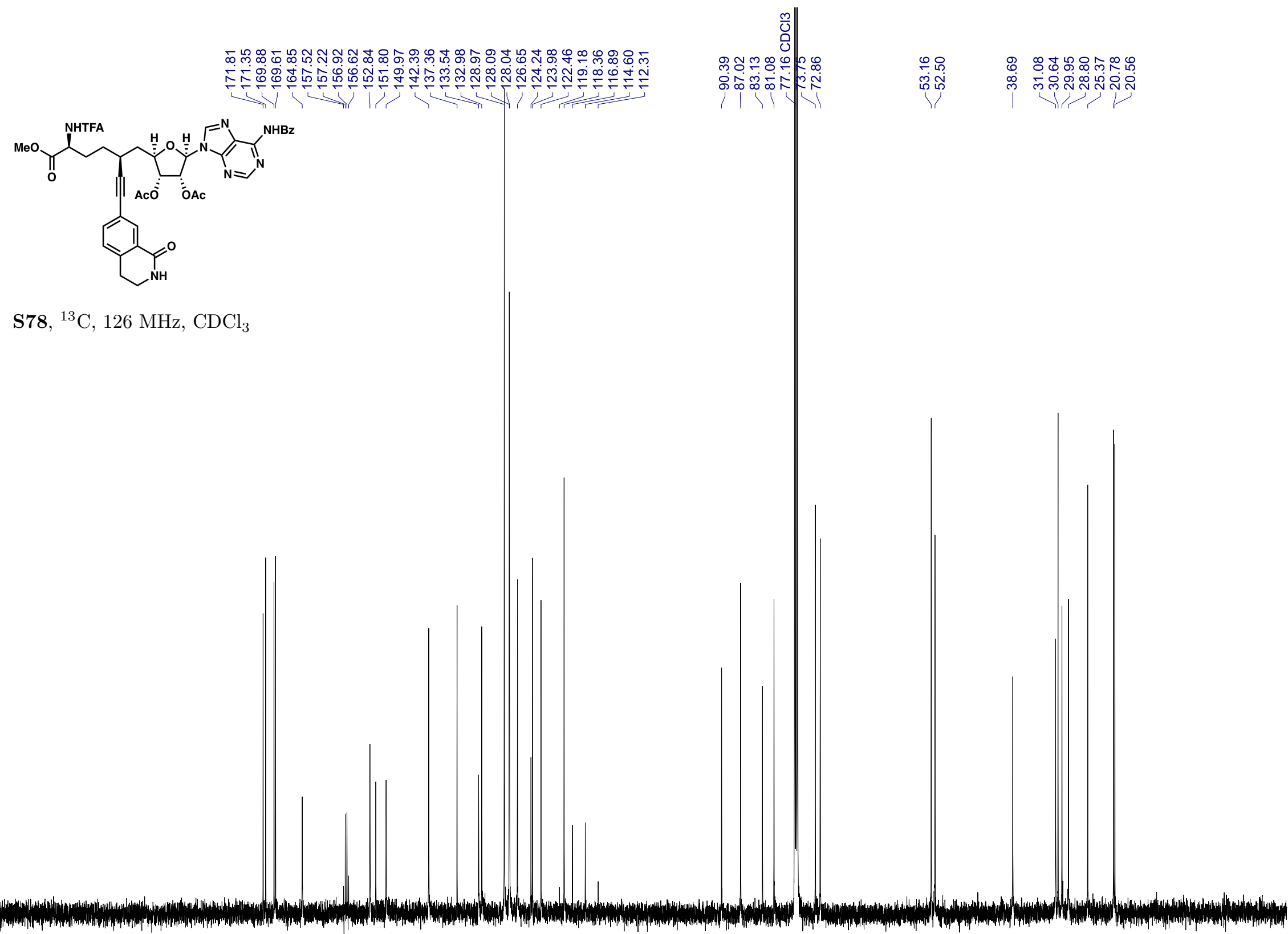

$210 \quad 200$ 


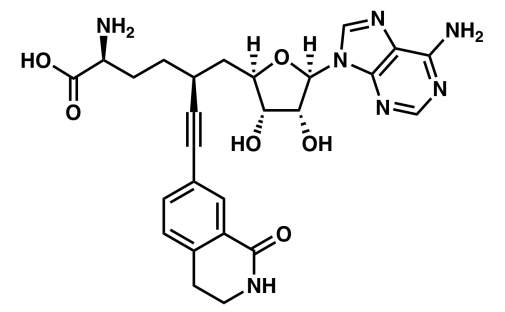

34, ${ }^{1} \mathrm{H}, 600 \mathrm{MHz}, \mathbf{C D}_{\mathbf{3}} \mathbf{C N} / \mathrm{D}_{2} \mathrm{O} / d-\mathrm{TFA}$

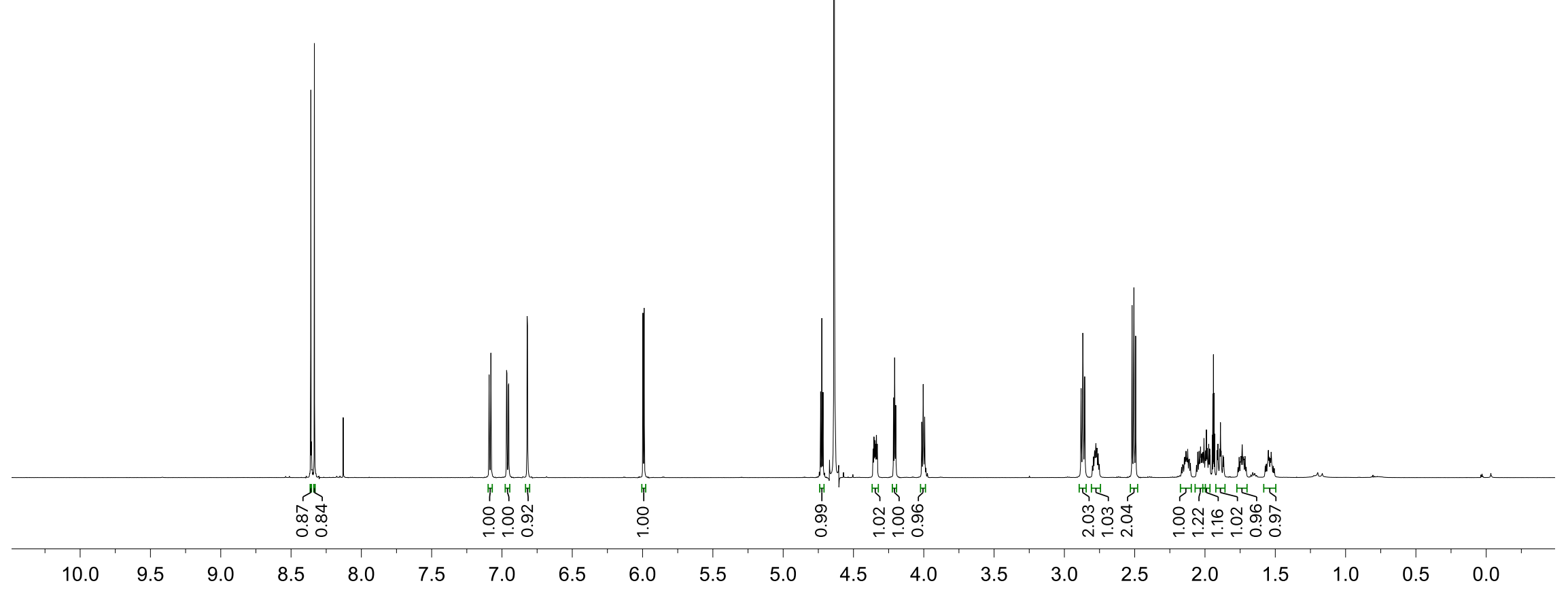




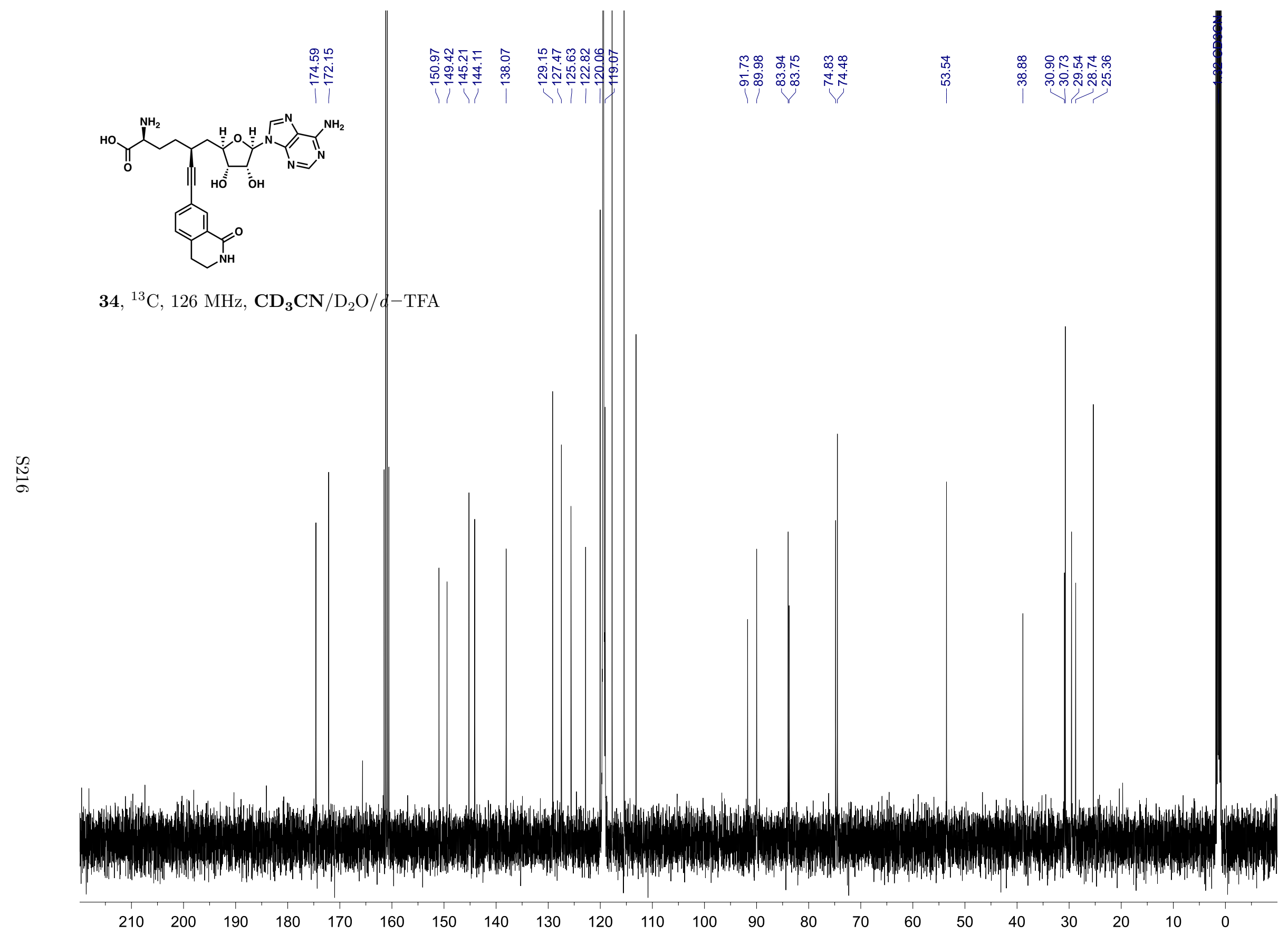




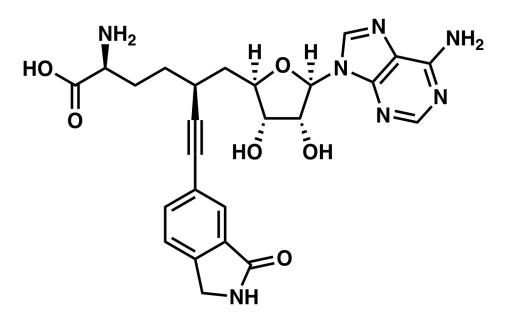

35, ${ }^{1} \mathrm{H}, 600 \mathrm{MHz}, \mathbf{C D}_{\mathbf{3}} \mathbf{C N} / \mathrm{D}_{2} \mathrm{O} / d-\mathrm{TFA}$ 


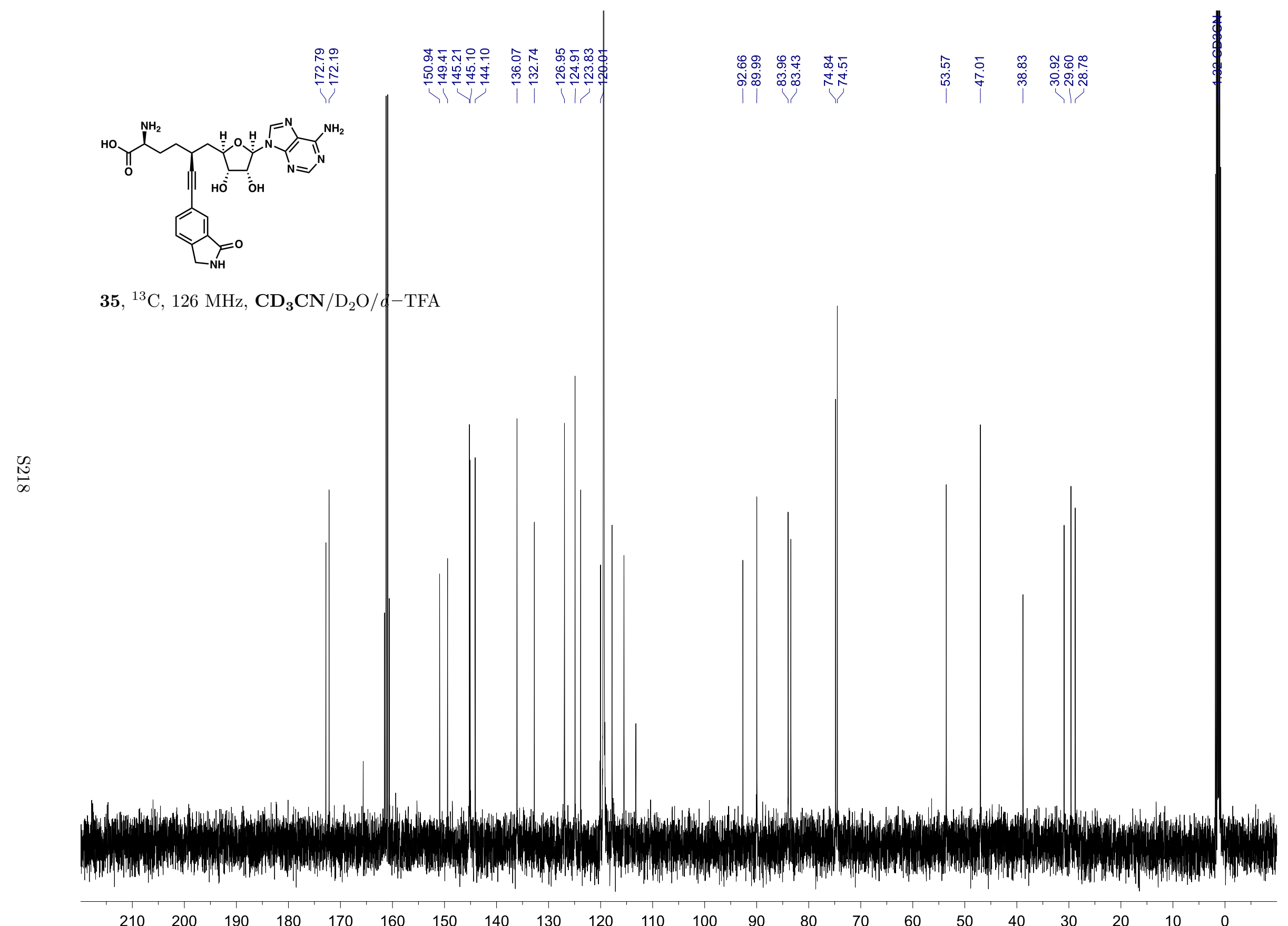

$\begin{array}{llllll}210 & 200 & 190 & 180 & 170 & 160\end{array}$

$\begin{array}{lll}140 & 130 & 120\end{array}$

$80 \quad 70$

$60 \quad 50$

30

0 


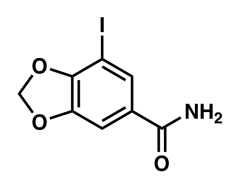

S80, ${ }^{1} \mathrm{H}, 500 \mathrm{MHz}, \mathrm{CD}_{3} \mathrm{OD}$

$\underset{\substack{N \\ 0}}{\stackrel{N}{0}}$

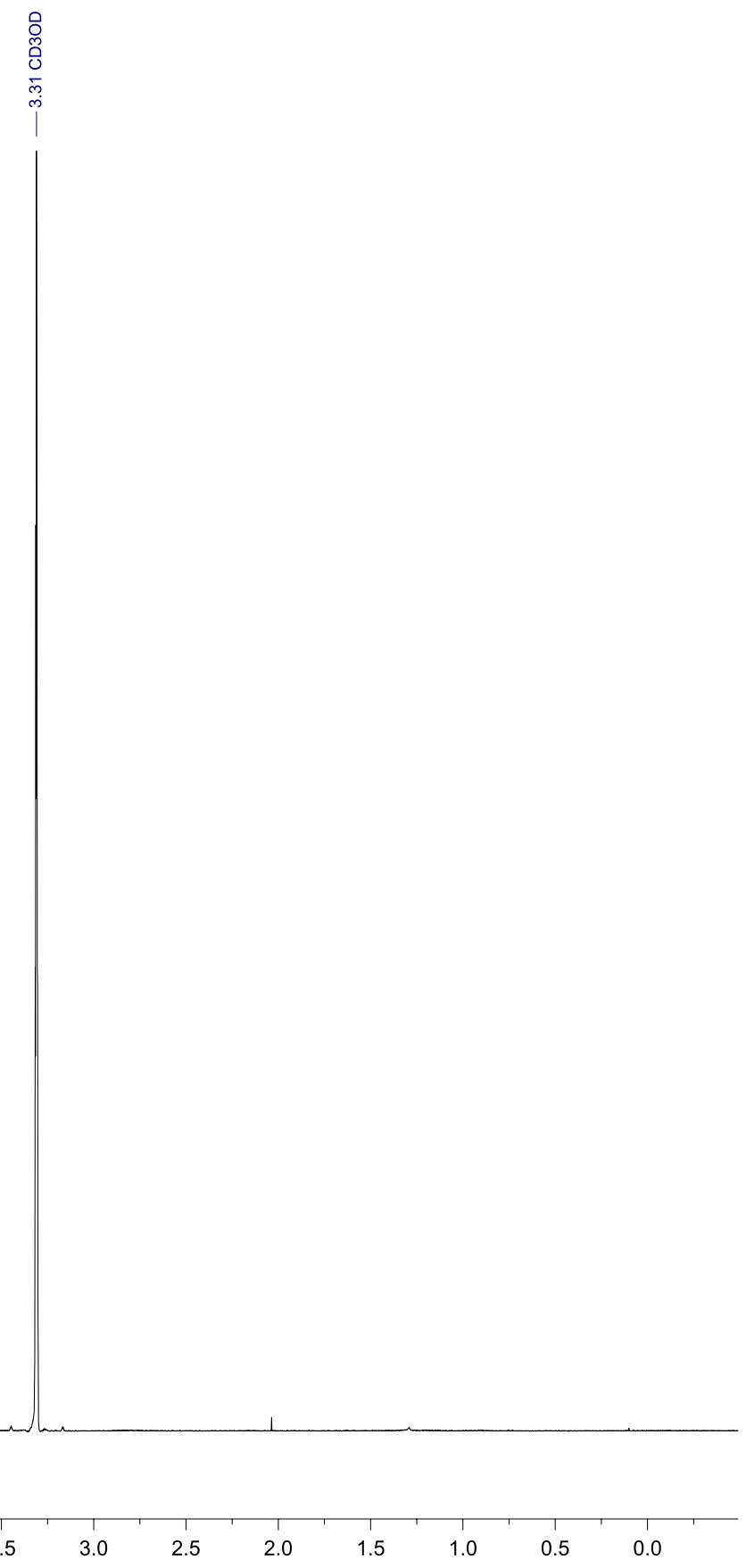




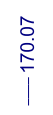

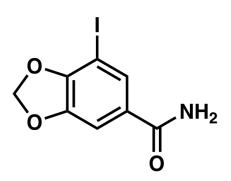

S80, ${ }^{13} \mathrm{C}, 101 \mathrm{MHz}, \mathrm{CD}_{3} \mathrm{OD}$

柋
하요

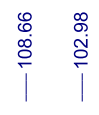

i
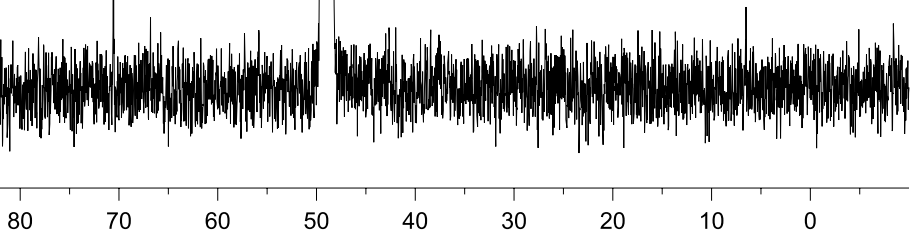

50

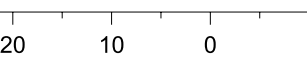




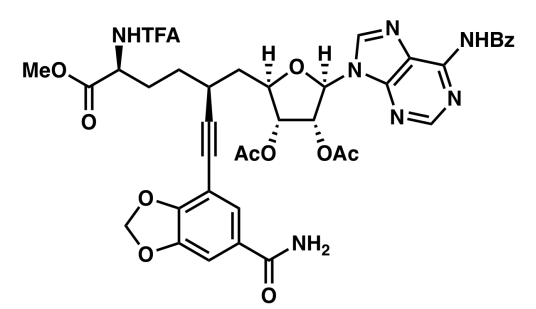

S81, ${ }^{1} \mathrm{H}, 500 \mathrm{MHz}, \mathrm{CD}_{3} \mathrm{OD}$

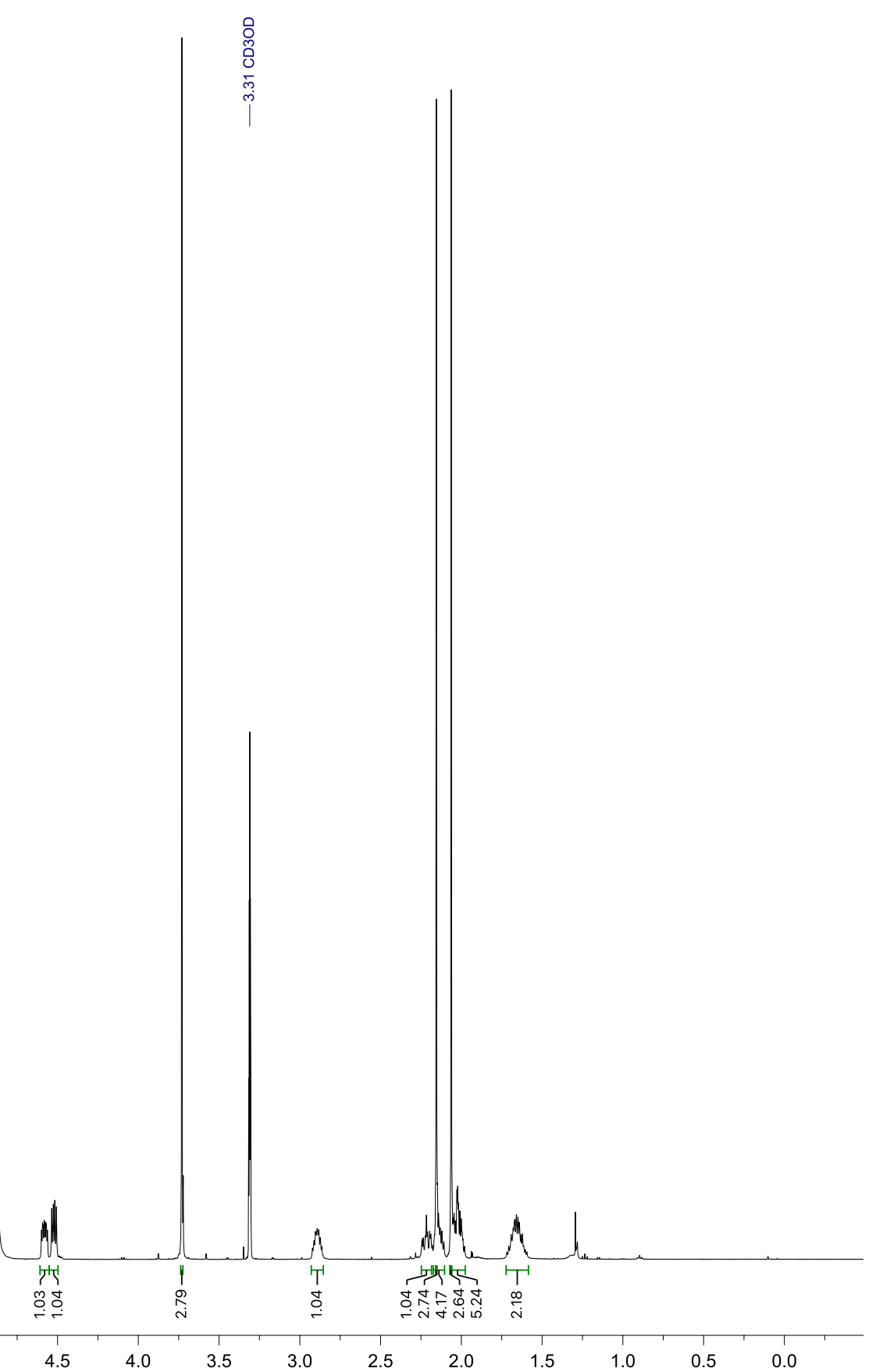




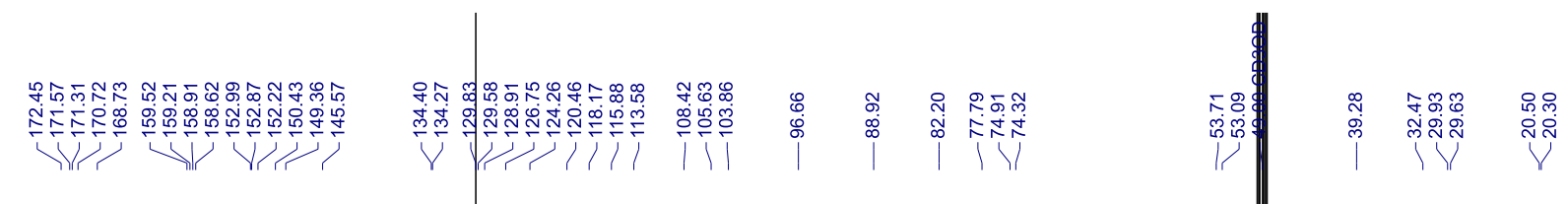

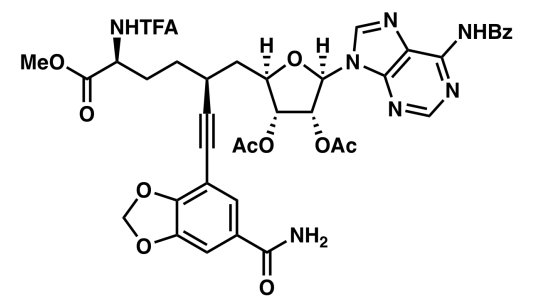

S81, ${ }^{13} \mathrm{C}, 126 \mathrm{MHz}, \mathrm{CD}_{3} \mathrm{OD}$ 


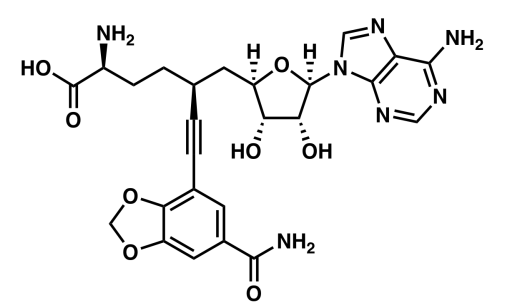

36, ${ }^{1} \mathrm{H}, 600 \mathrm{MHz}, \mathbf{C D}_{\mathbf{3}} \mathbf{C N} / \mathrm{D}_{2} \mathrm{O}$

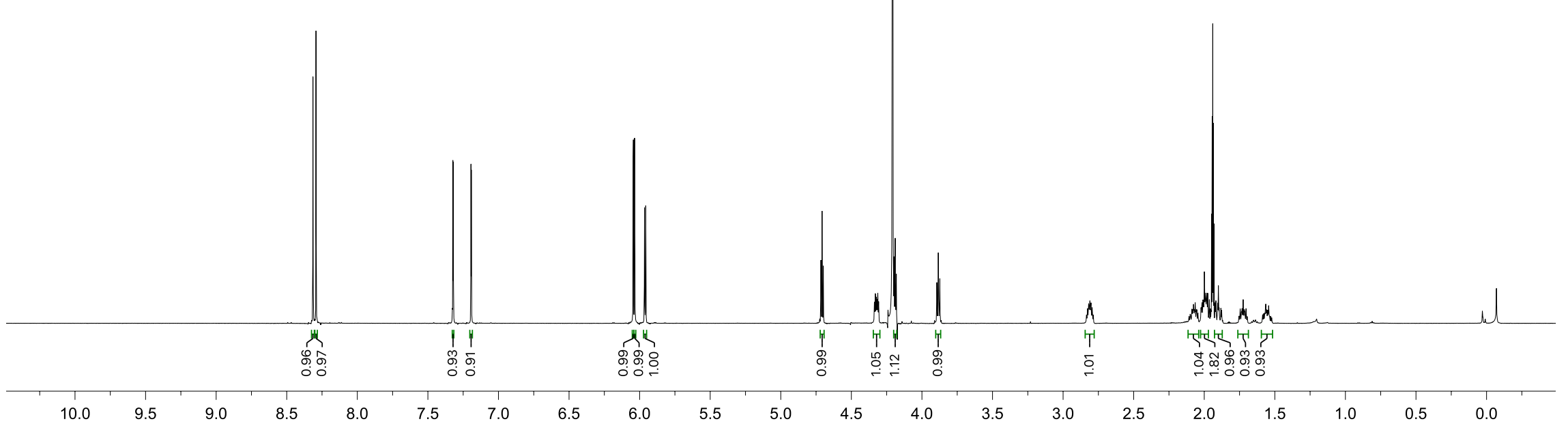




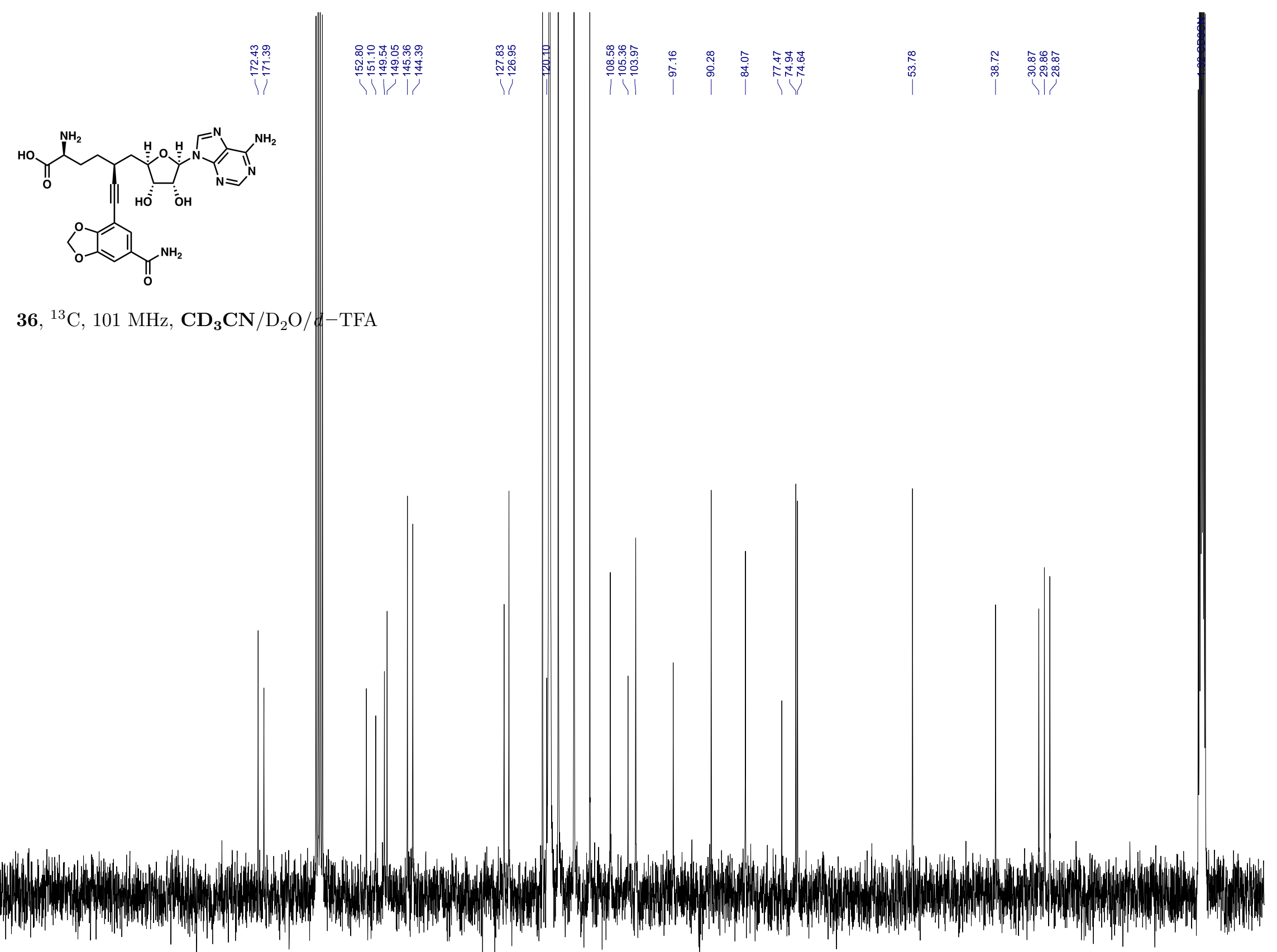

190

180

$170 \quad 160$

150

$130 \quad 120$

$110 \quad 100$

$90 \quad 80 \quad 70$

$60 \quad 50$

$40 \quad 30$

20

$10 \quad 0$ 


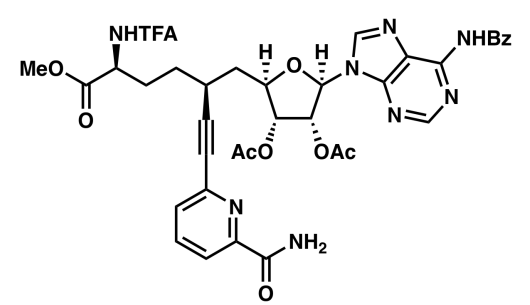

S82, ${ }^{1} \mathrm{H}, 600 \mathrm{MHz}, \mathrm{CDCl}_{3}$ 
లై

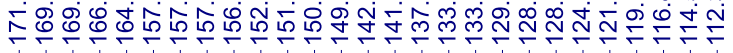

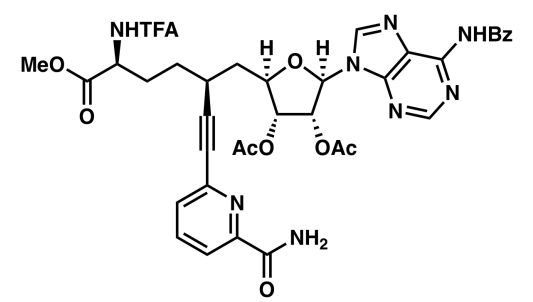

$\mathbf{S 8 2},{ }^{13} \mathrm{C}, 126 \mathrm{MHz}, \mathrm{CDCl}_{3}$

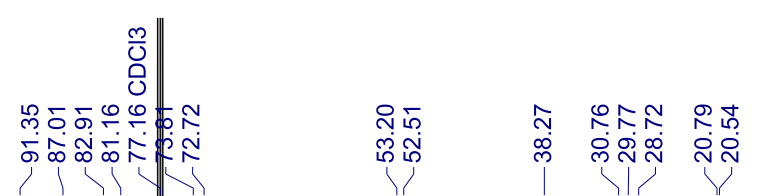

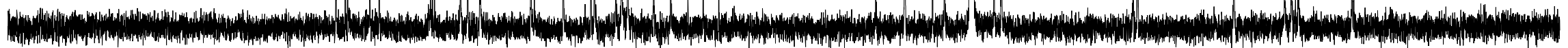




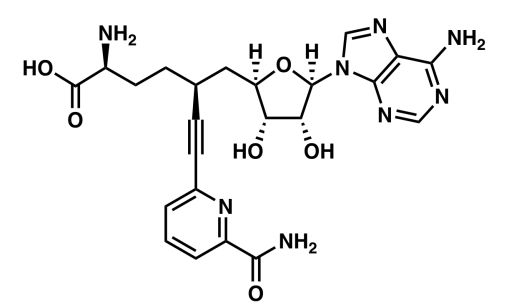

37, ${ }^{1} \mathrm{H}, 600 \mathrm{MHz}, \mathbf{C D}_{\mathbf{3}} \mathbf{C N} / \mathrm{D}_{2} \mathrm{O} / d-\mathrm{TFA}$

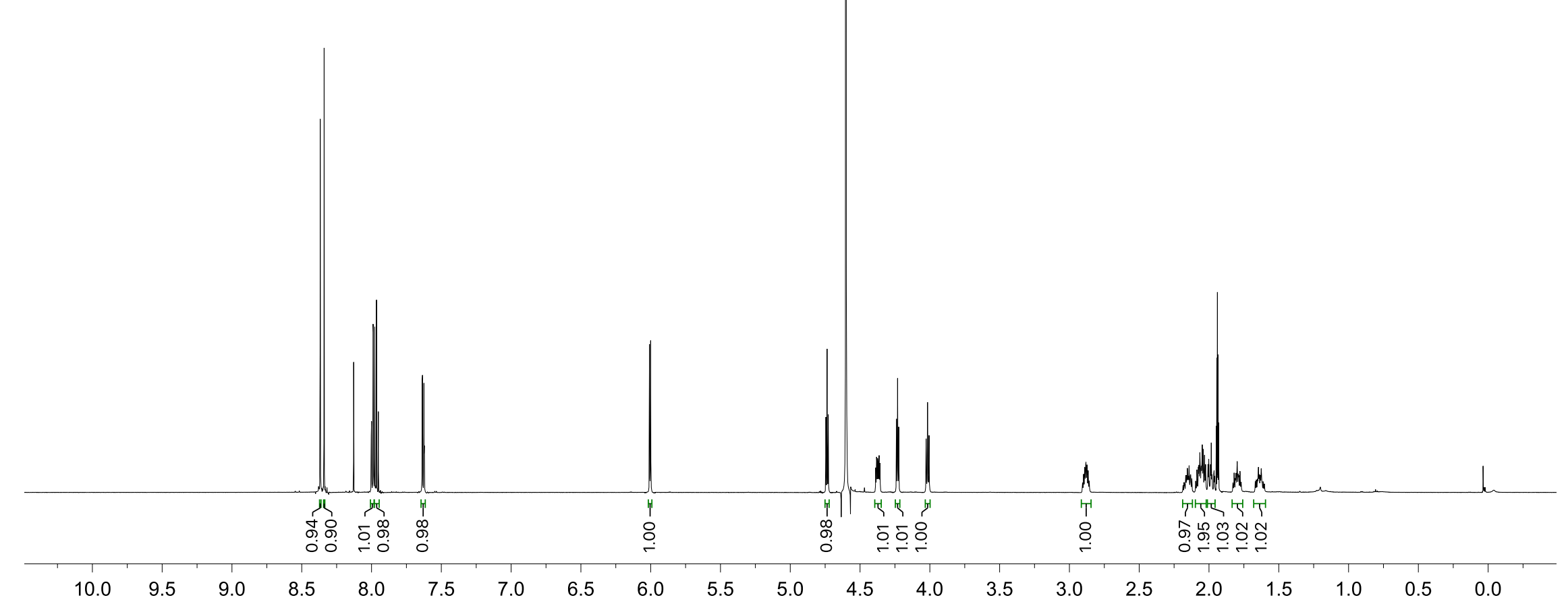




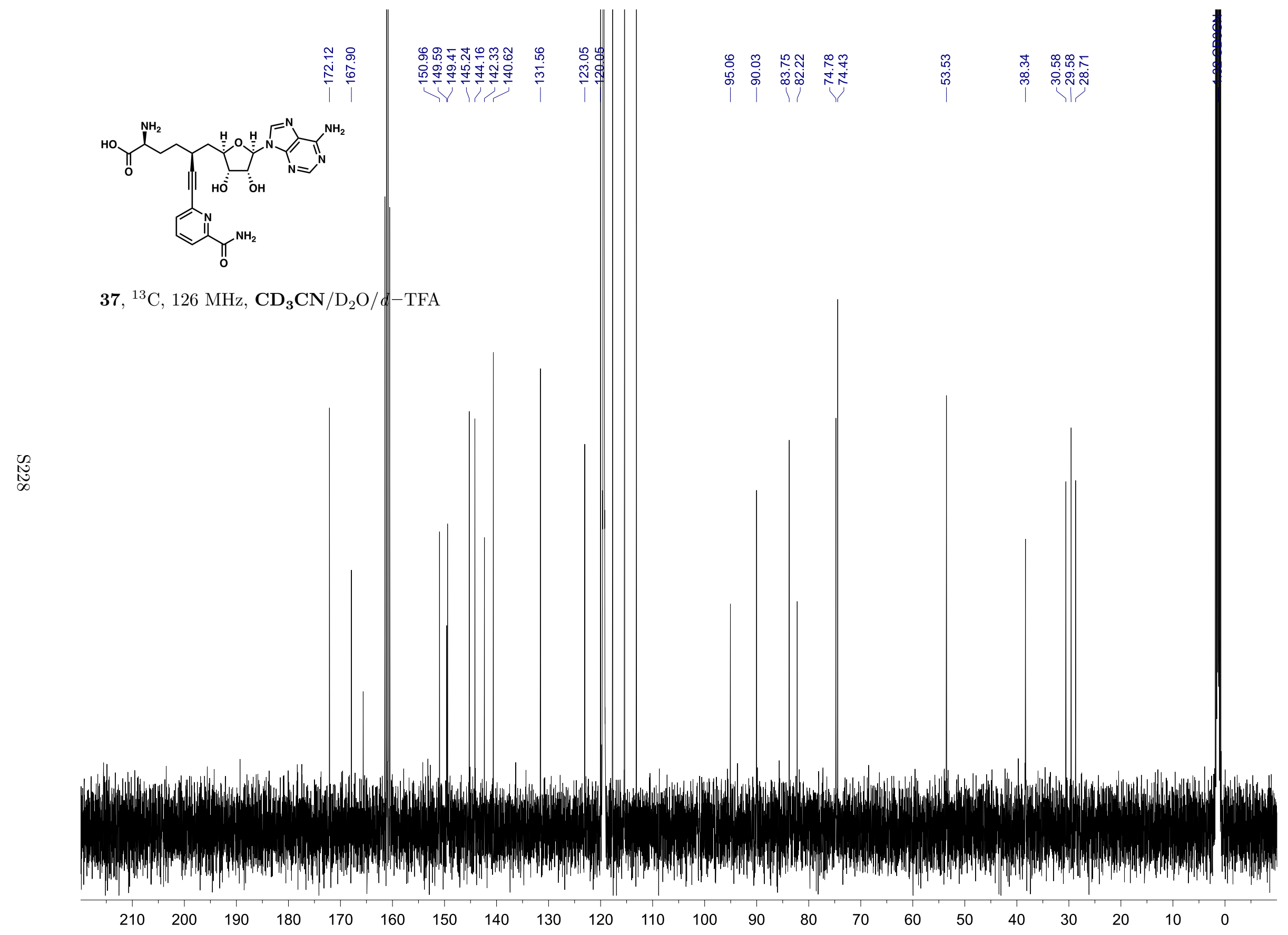




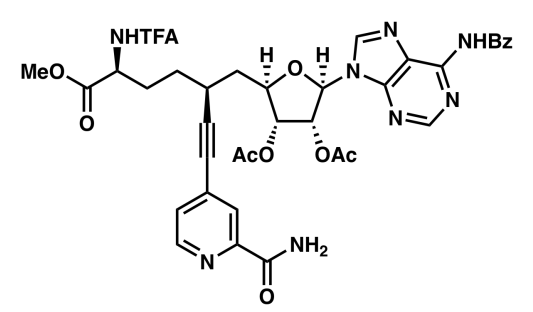

S83, ${ }^{1} \mathrm{H}, 600 \mathrm{MHz}, \mathrm{CDCl}_{3}$

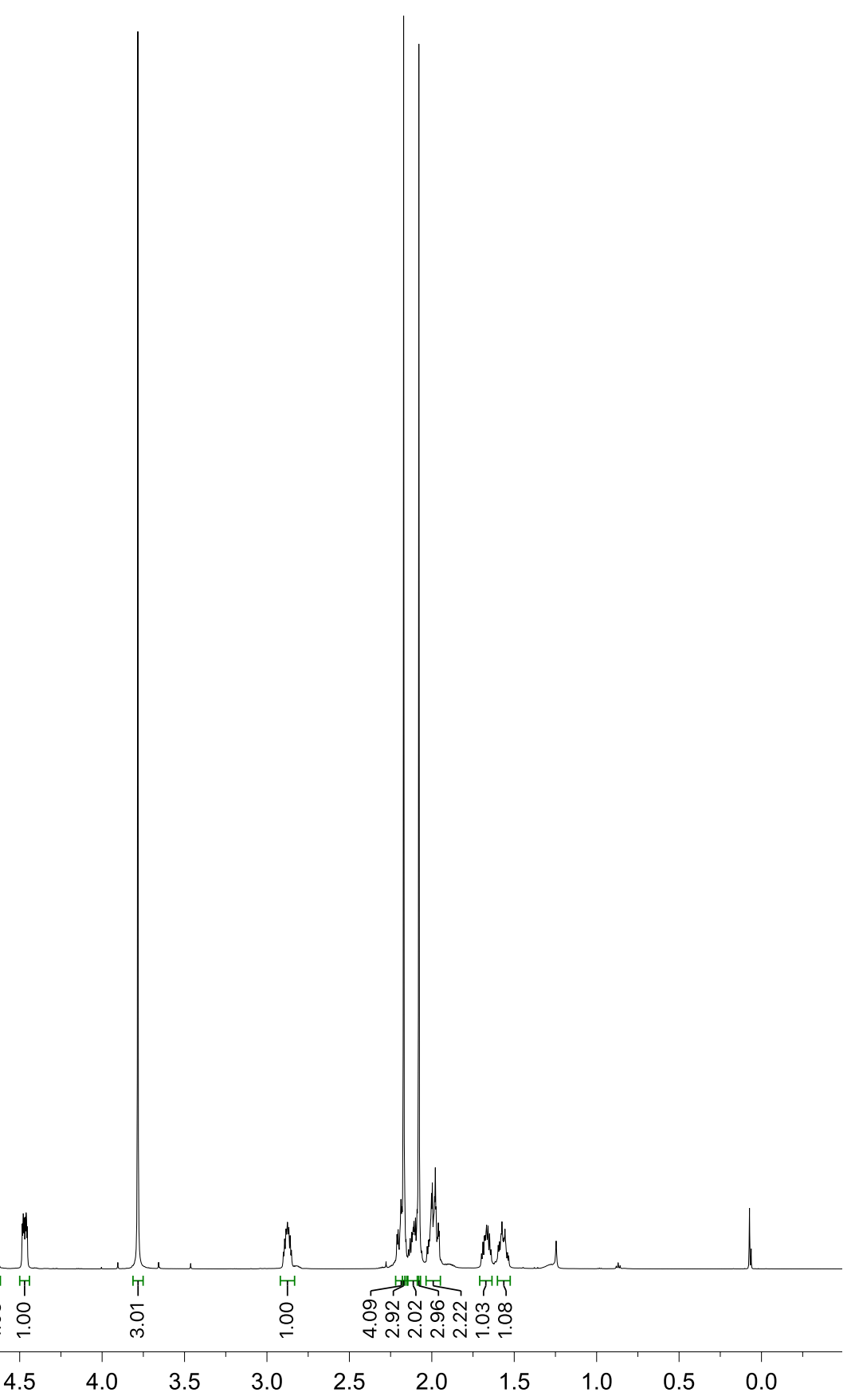




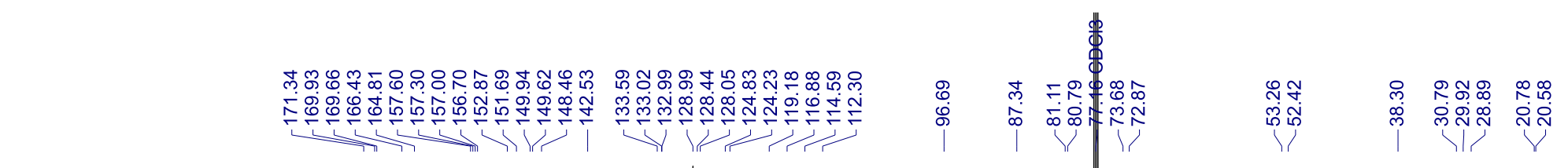

S83, ${ }^{13} \mathrm{C}, 126 \mathrm{MHz}, \mathrm{CDCl}_{3}$

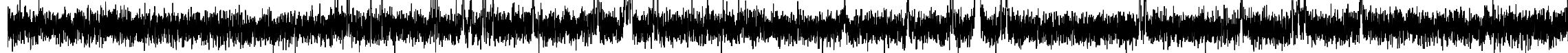




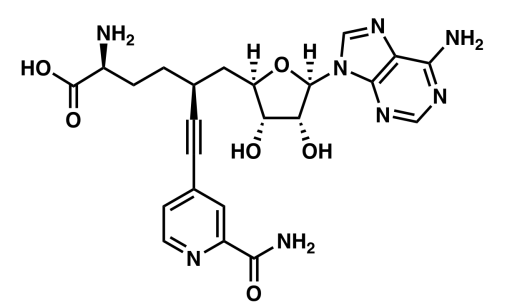

38, ${ }^{1} \mathrm{H}, 600 \mathrm{MHz}, \mathbf{C D}_{\mathbf{3}} \mathbf{C N} / \mathrm{D}_{2} \mathrm{O} / d-\mathrm{TFA}$

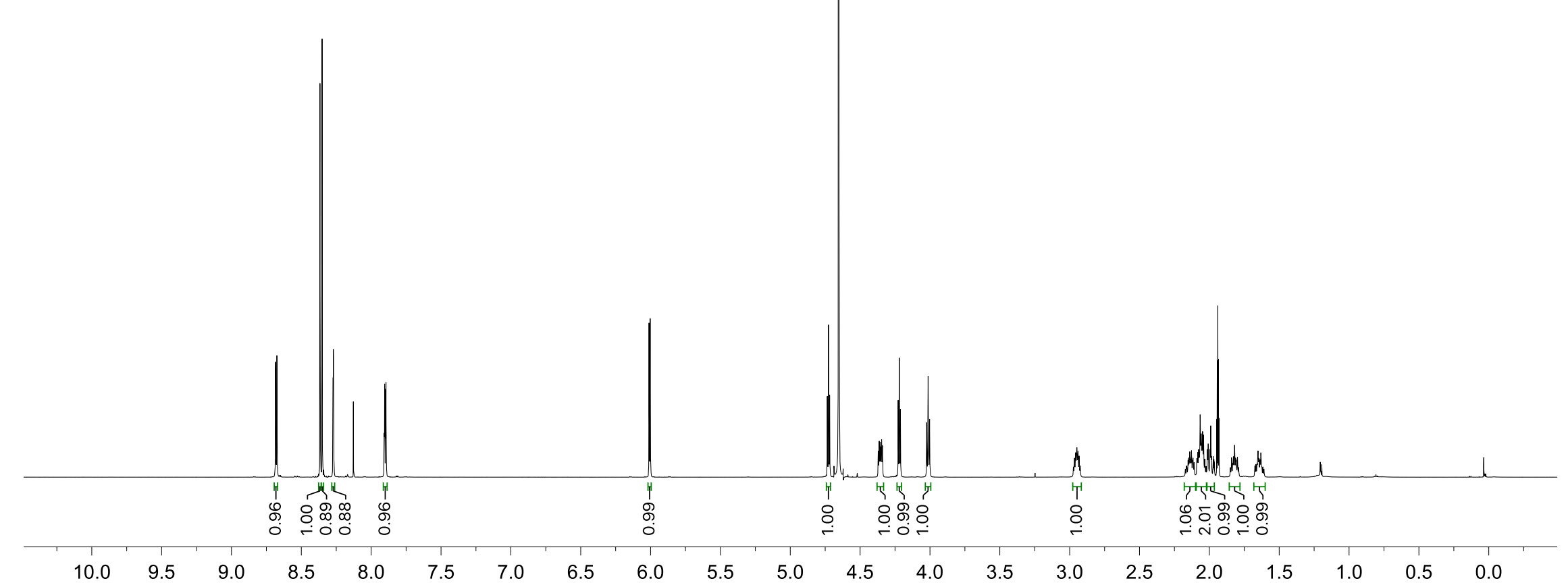




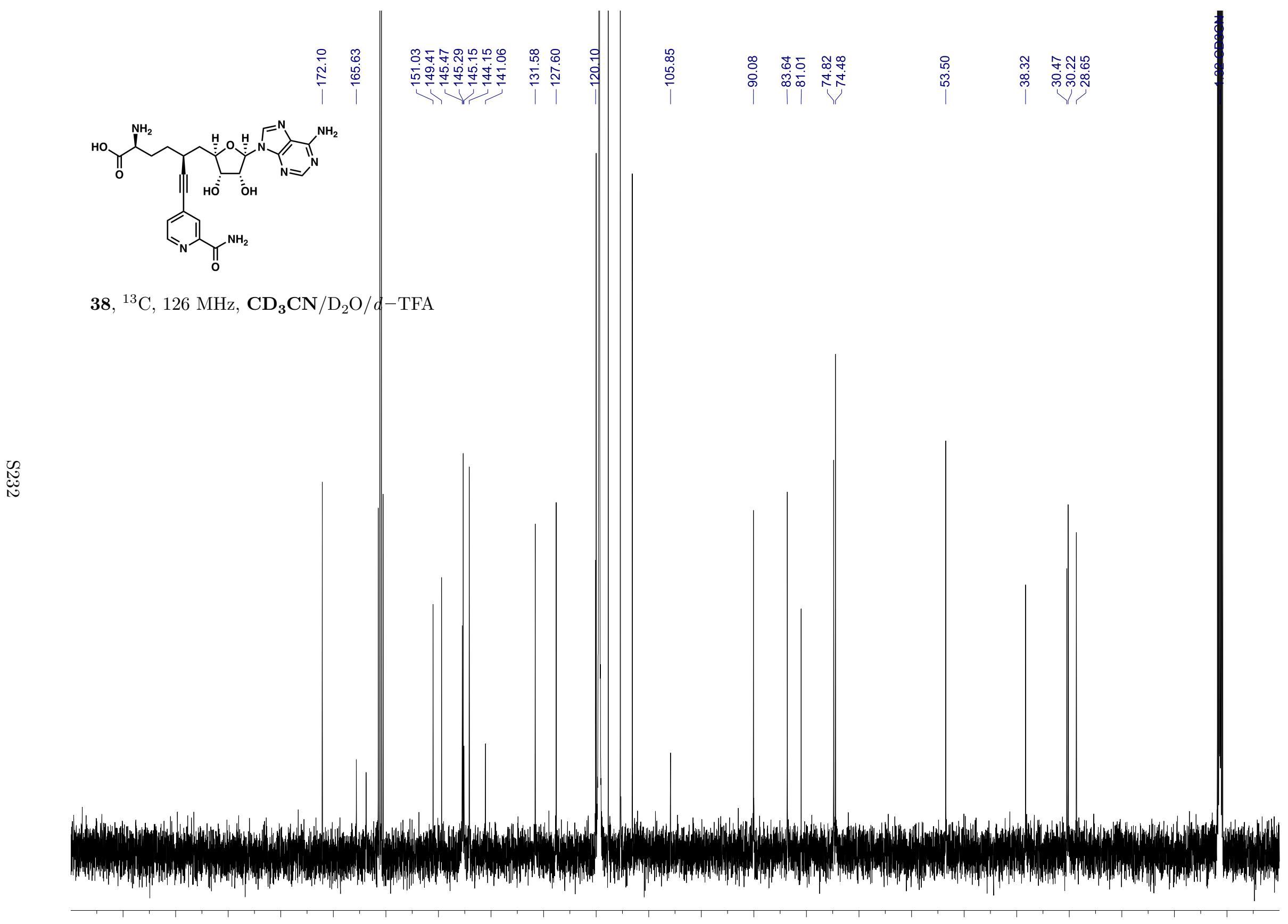

$\begin{array}{lllllll}210 & 200 & 190 & 180 & 170 & 160 & 150\end{array}$

$140 \quad 130$

8070

$60 \quad 50$

$40 \quad 30$

2010

0 


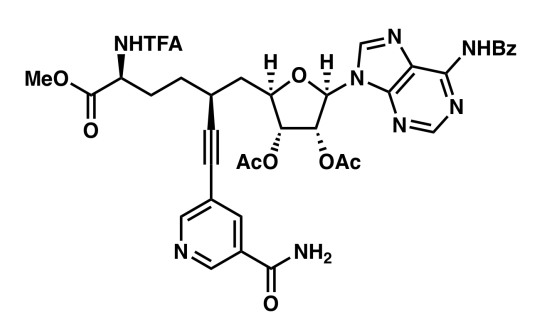

$\mathbf{S 8 4},{ }^{1} \mathrm{H}, 600 \mathrm{MHz}, \mathrm{CDCl}_{3}$

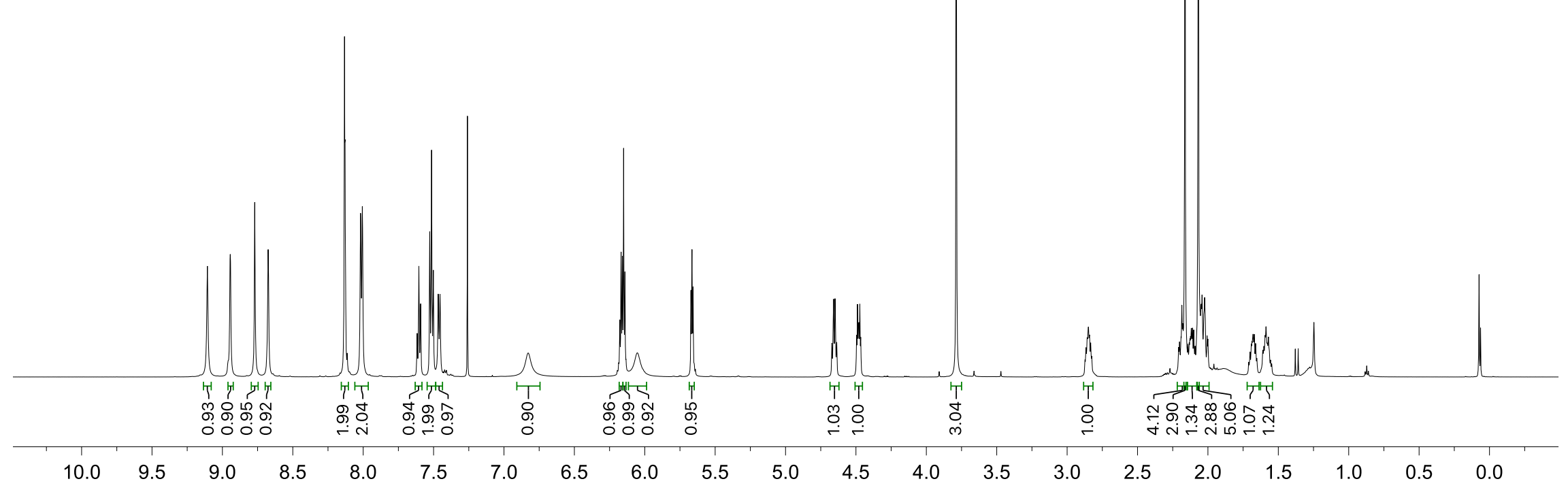



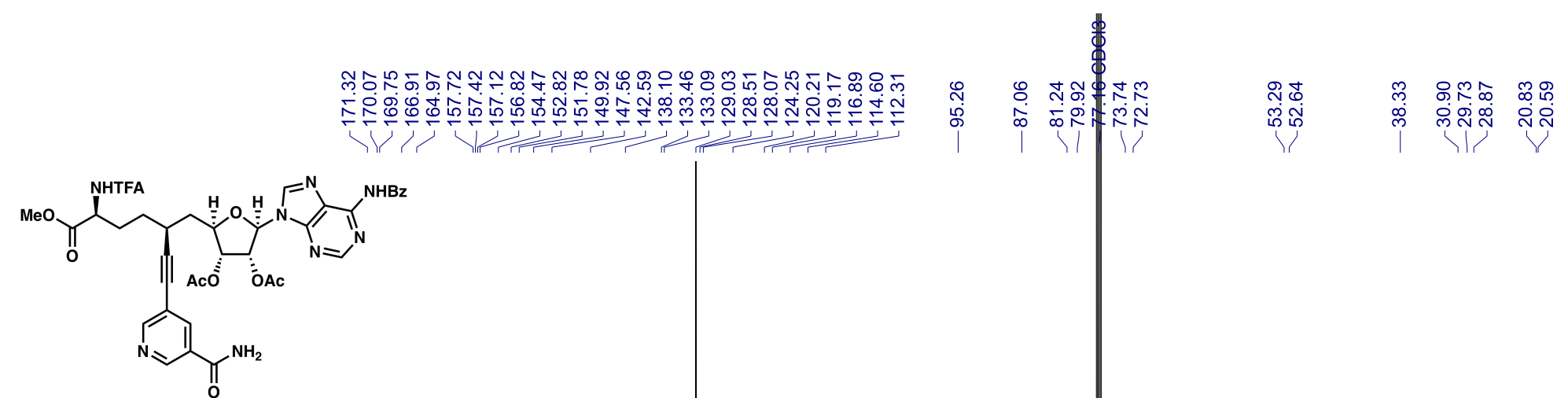

S84, ${ }^{13} \mathrm{C}, 126 \mathrm{MHz}, \mathrm{CDCl}_{3}$

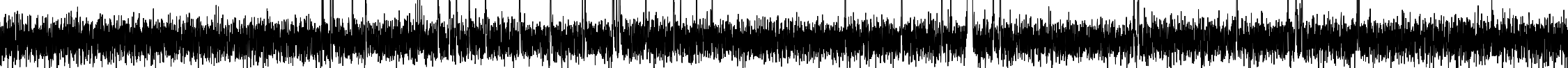




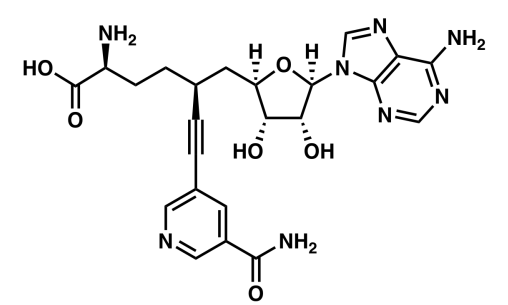

39, ${ }^{1} \mathrm{H}, 500 \mathrm{MHz}, \mathbf{C D}_{\mathbf{3}} \mathbf{C N} / \mathrm{D}_{2} \mathrm{O} / d-\mathrm{TFA}$

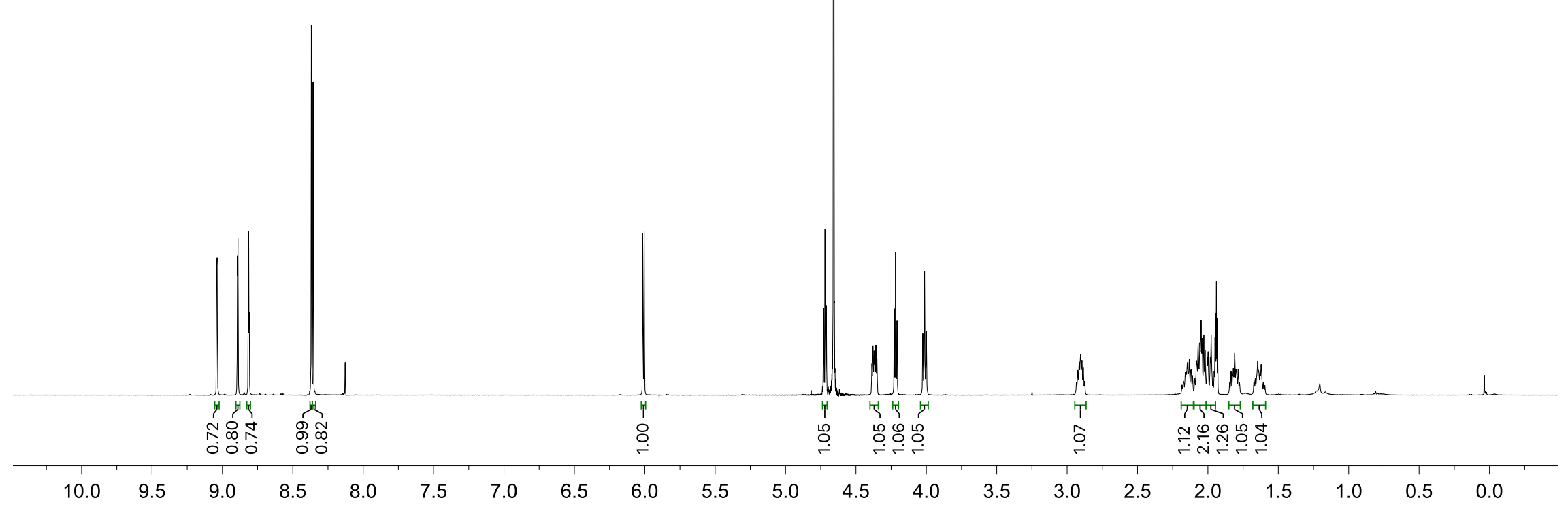




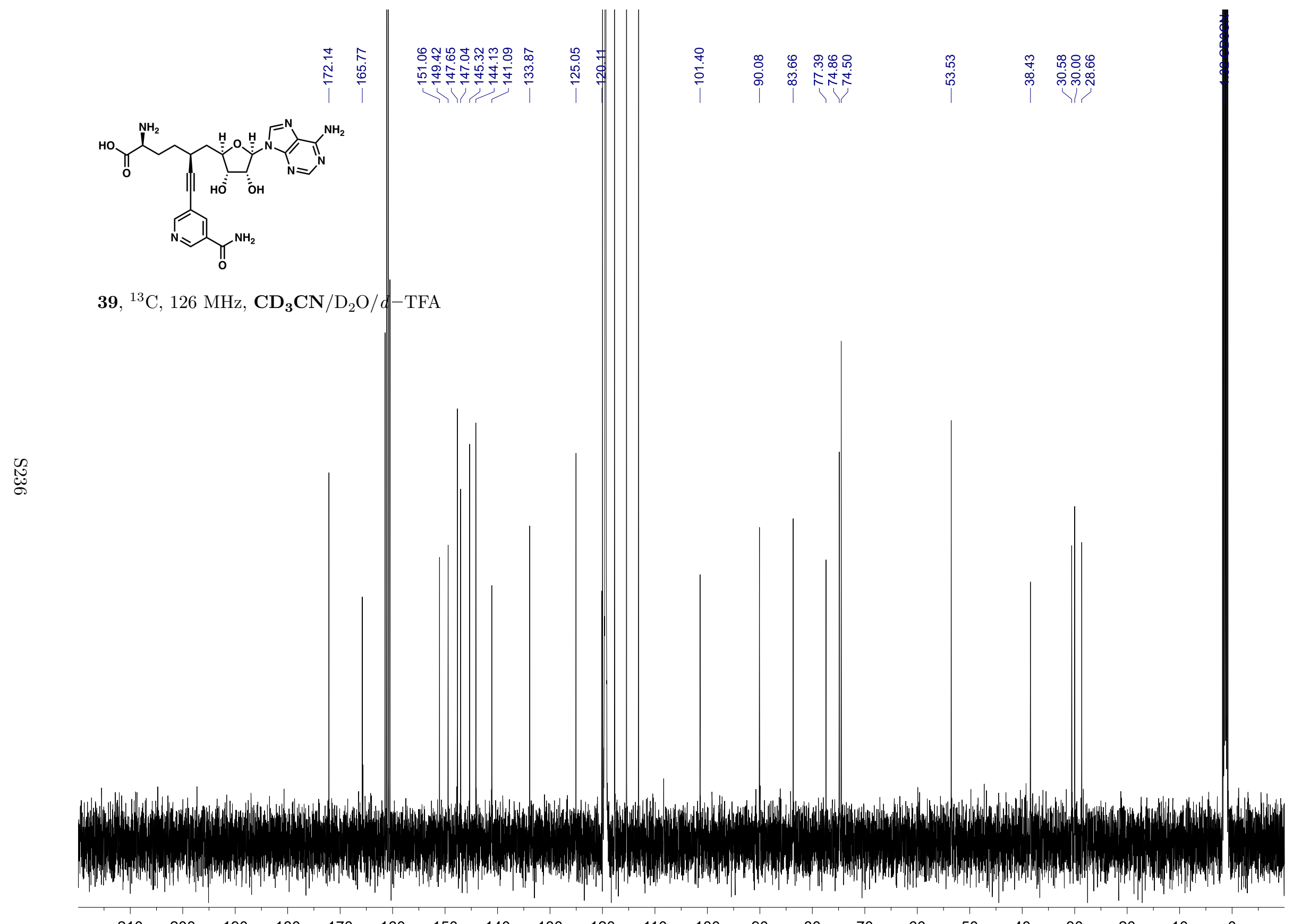

$130 \quad 120 \quad 110 \quad 100$

$90 \quad 80$

60

$40 \quad 30$ 


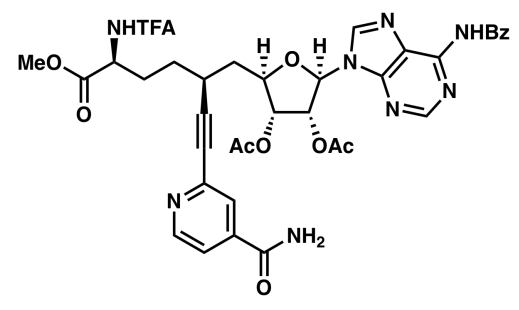

S85, ${ }^{1} \mathrm{H}, 600 \mathrm{MHz}, \mathrm{CDCl}_{3}$

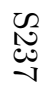

0
0
0
0
0

1

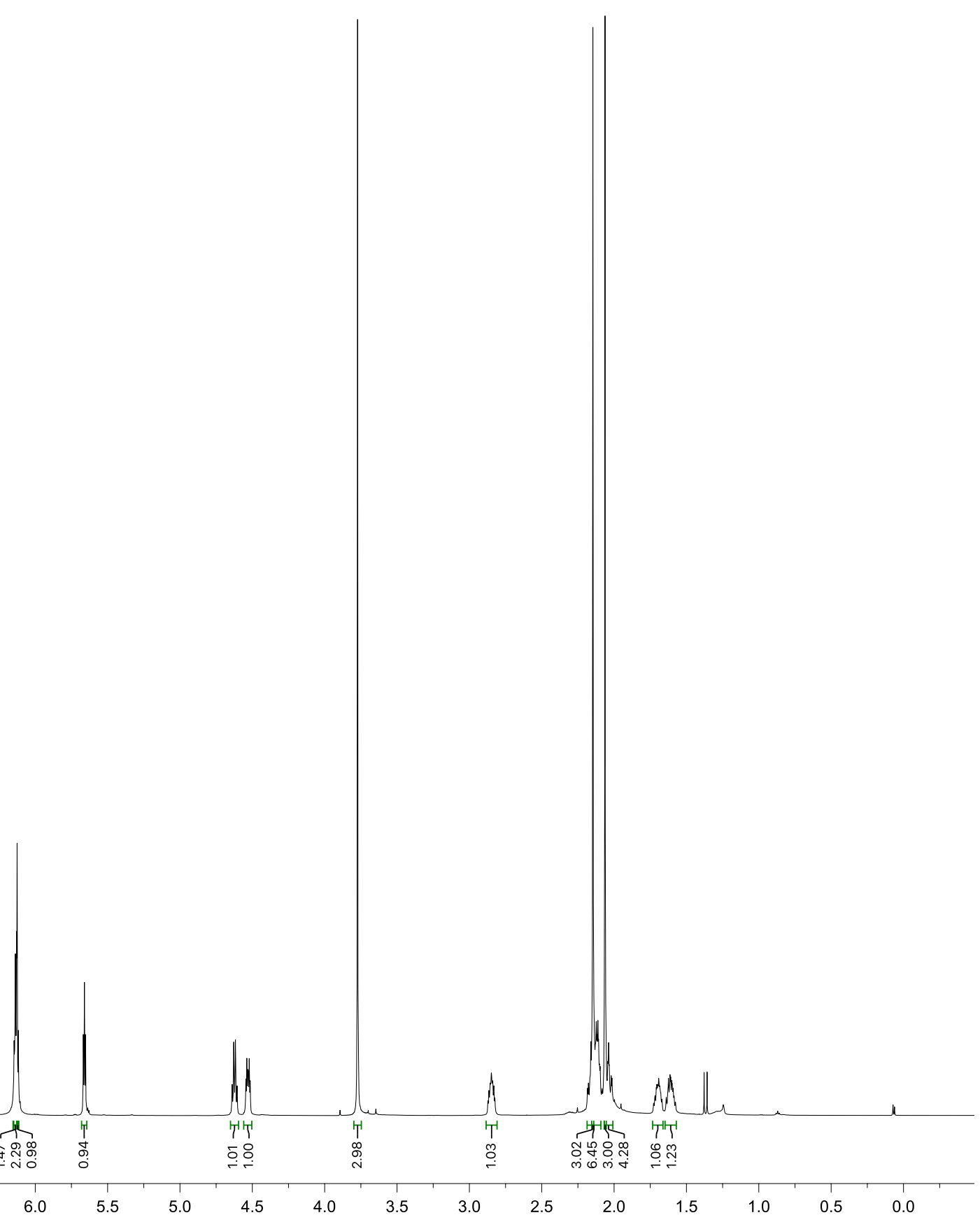




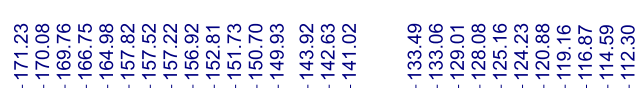

V।

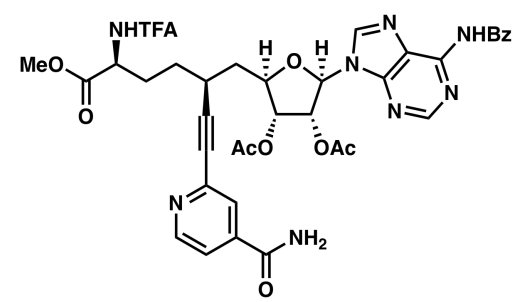

S85, ${ }^{13} \mathrm{C}, 126 \mathrm{MHz}, \mathrm{CDCl}_{3}$

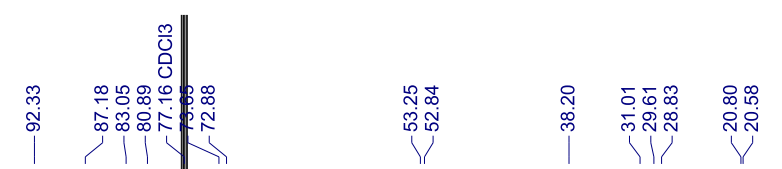

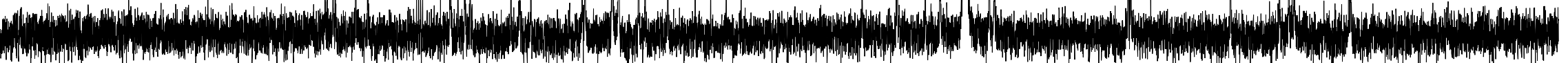

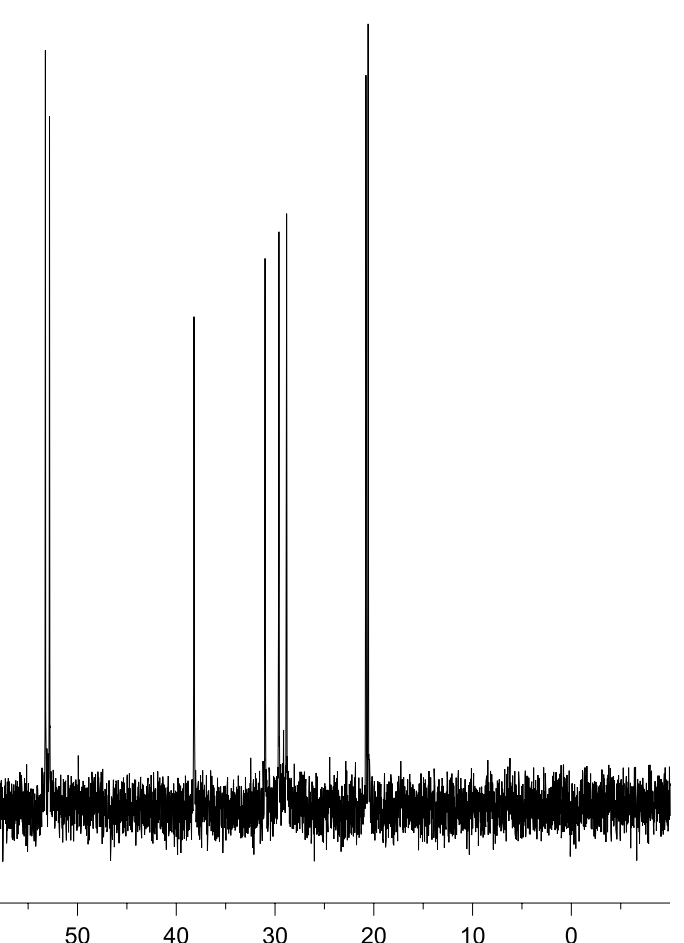




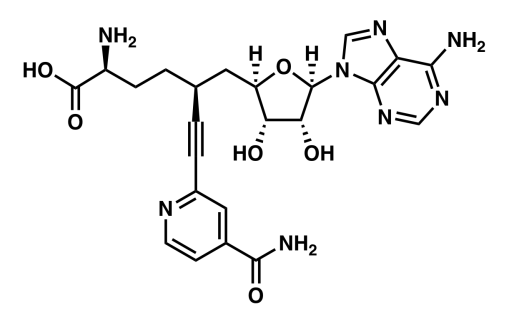

40, ${ }^{1} \mathrm{H}, 500 \mathrm{MHz}, \mathbf{C D}_{\mathbf{3}} \mathbf{C N} / \mathrm{D}_{2} \mathrm{O} / d-\mathrm{TFA}$

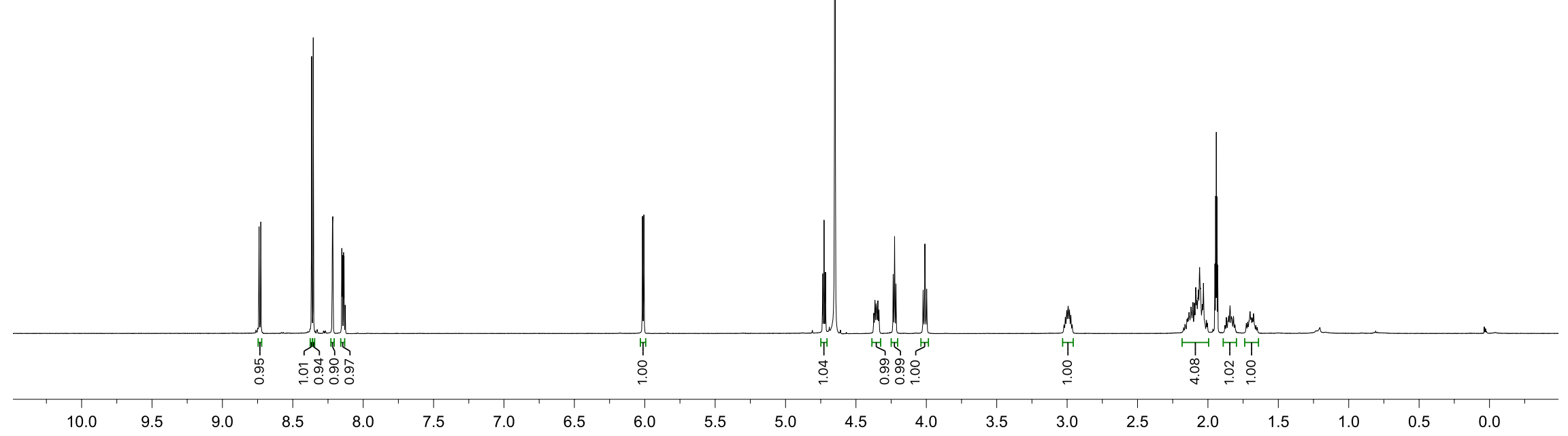




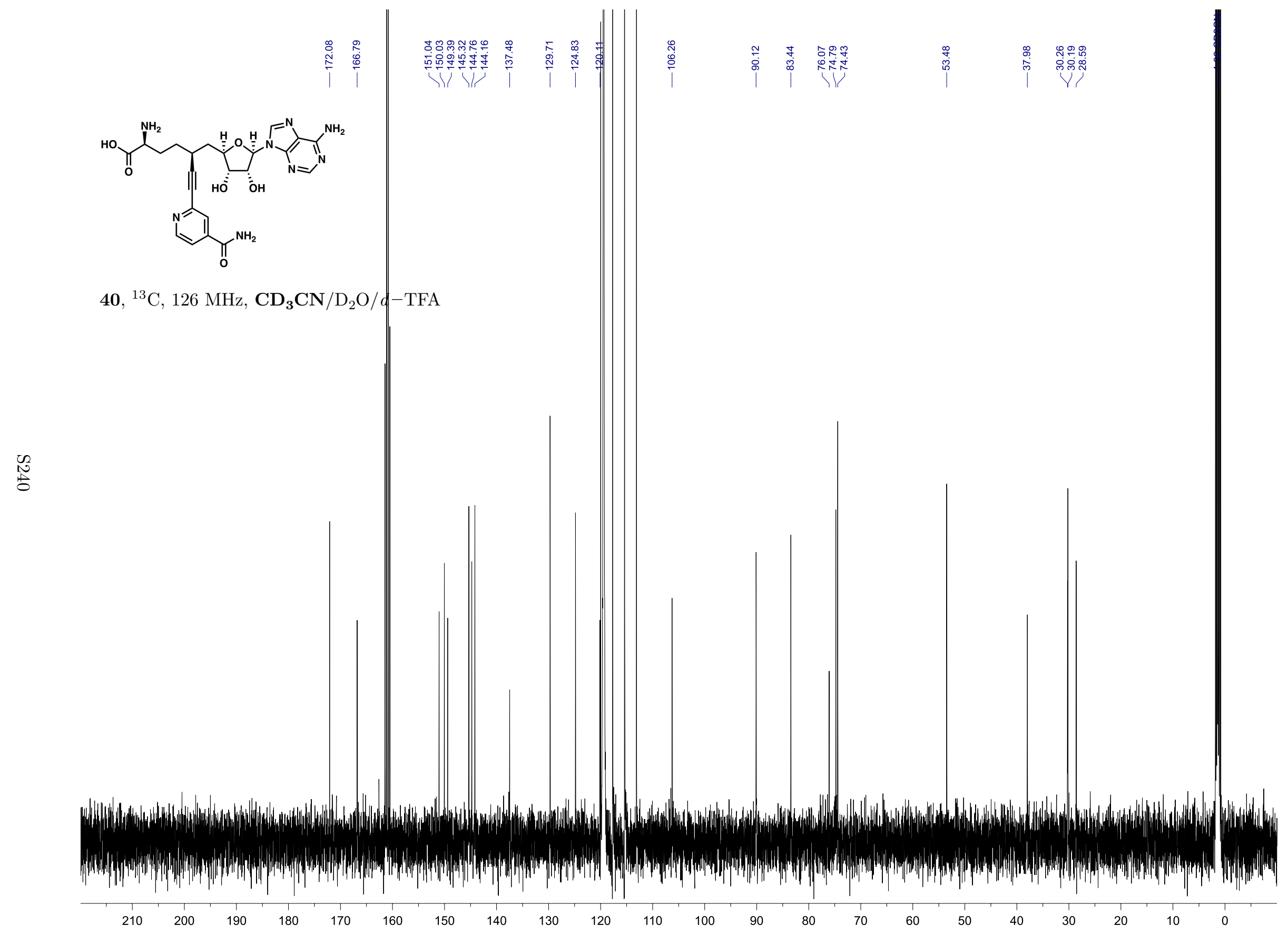




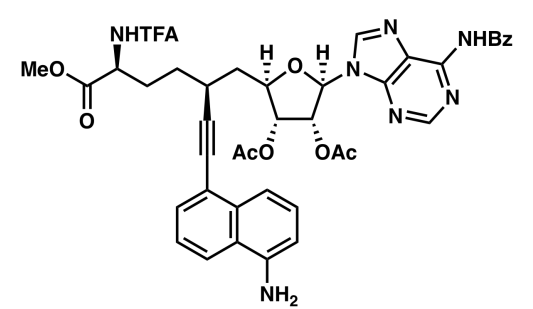

S86, ${ }^{1} \mathrm{H}, 600 \mathrm{MHz}, \mathrm{CDCl}_{3}$

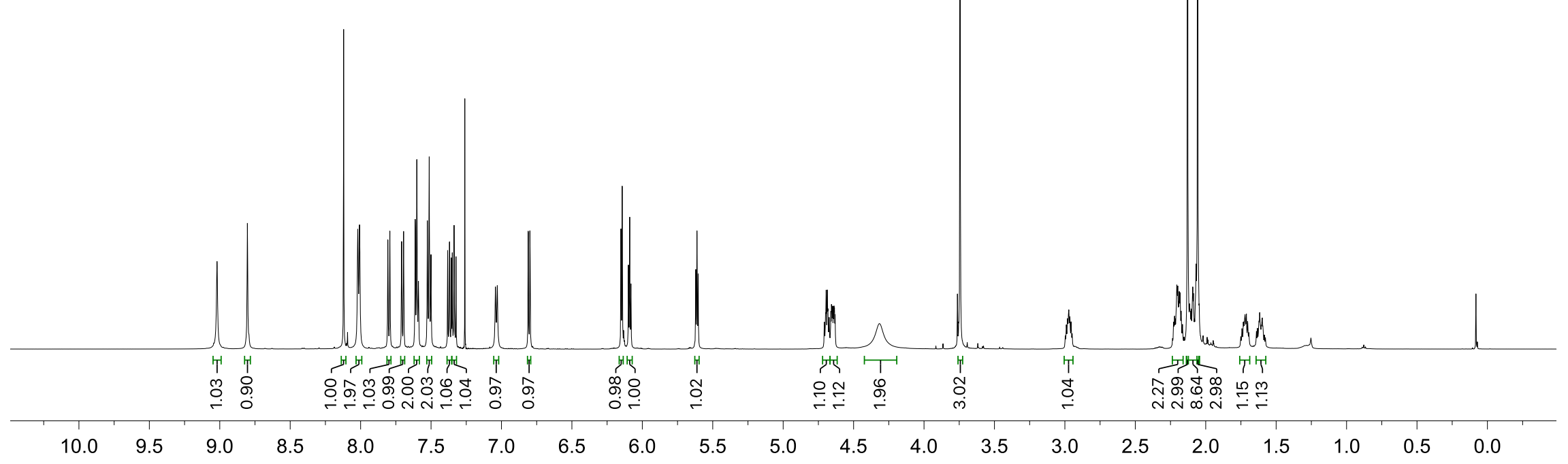



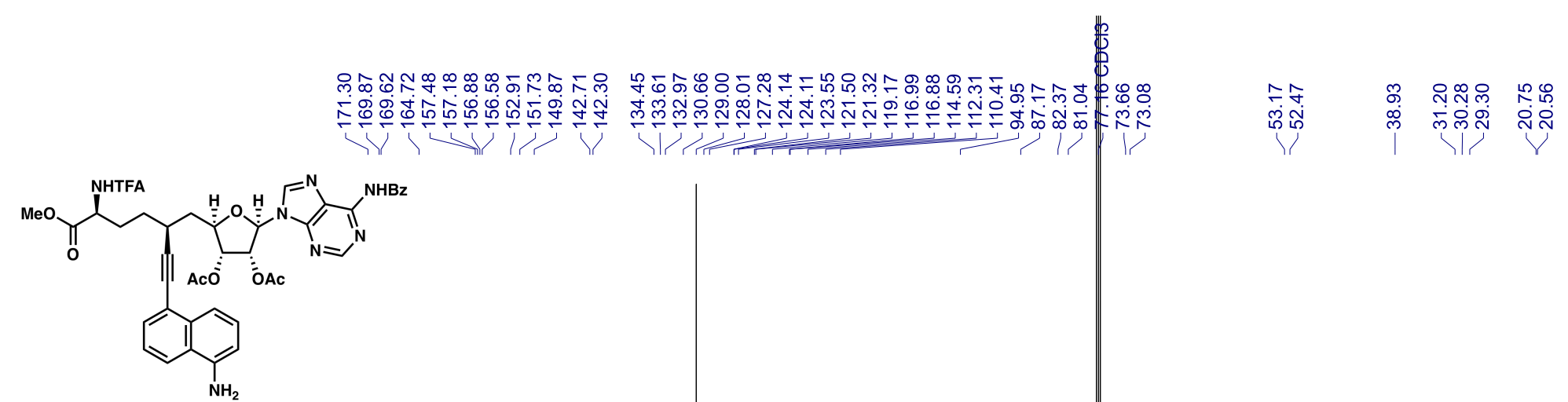

S86, ${ }^{13} \mathrm{C}, 126 \mathrm{MHz}, \mathrm{CDCl}_{3}$ 


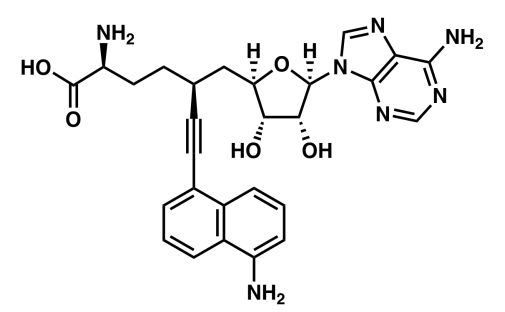

41, ${ }^{1} \mathrm{H}, 600 \mathrm{MHz}, \mathbf{C D}_{\mathbf{3}} \mathbf{C N} / \mathrm{D}_{2} \mathrm{O} / d-\mathrm{TFA}$

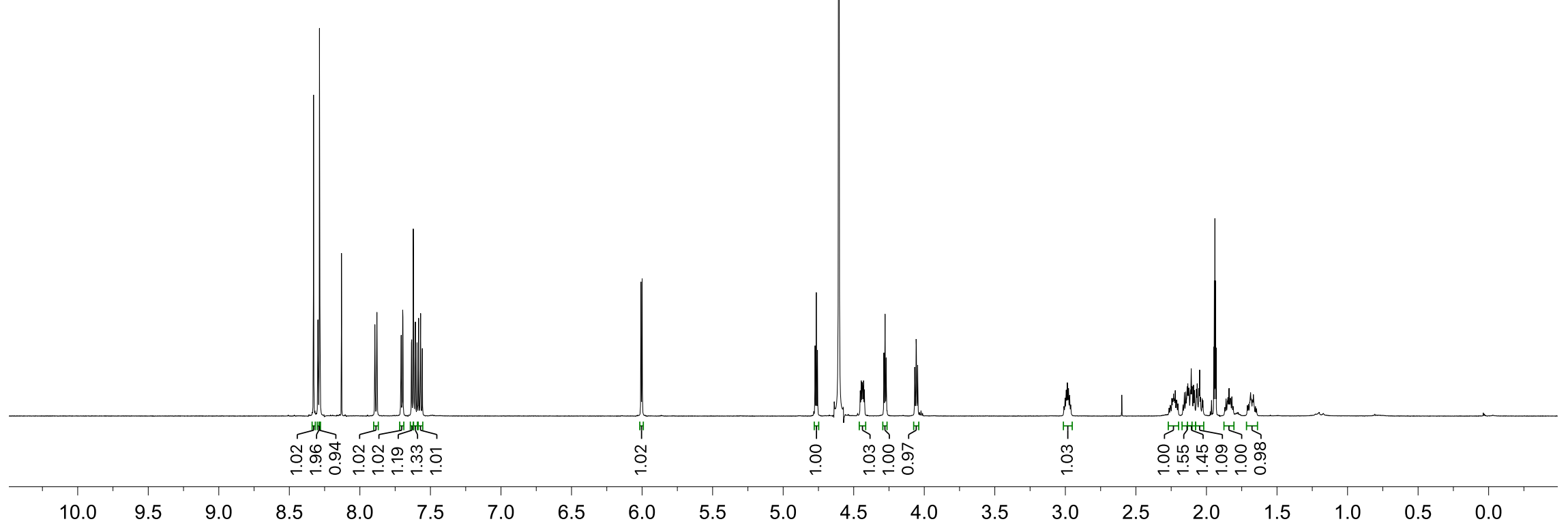




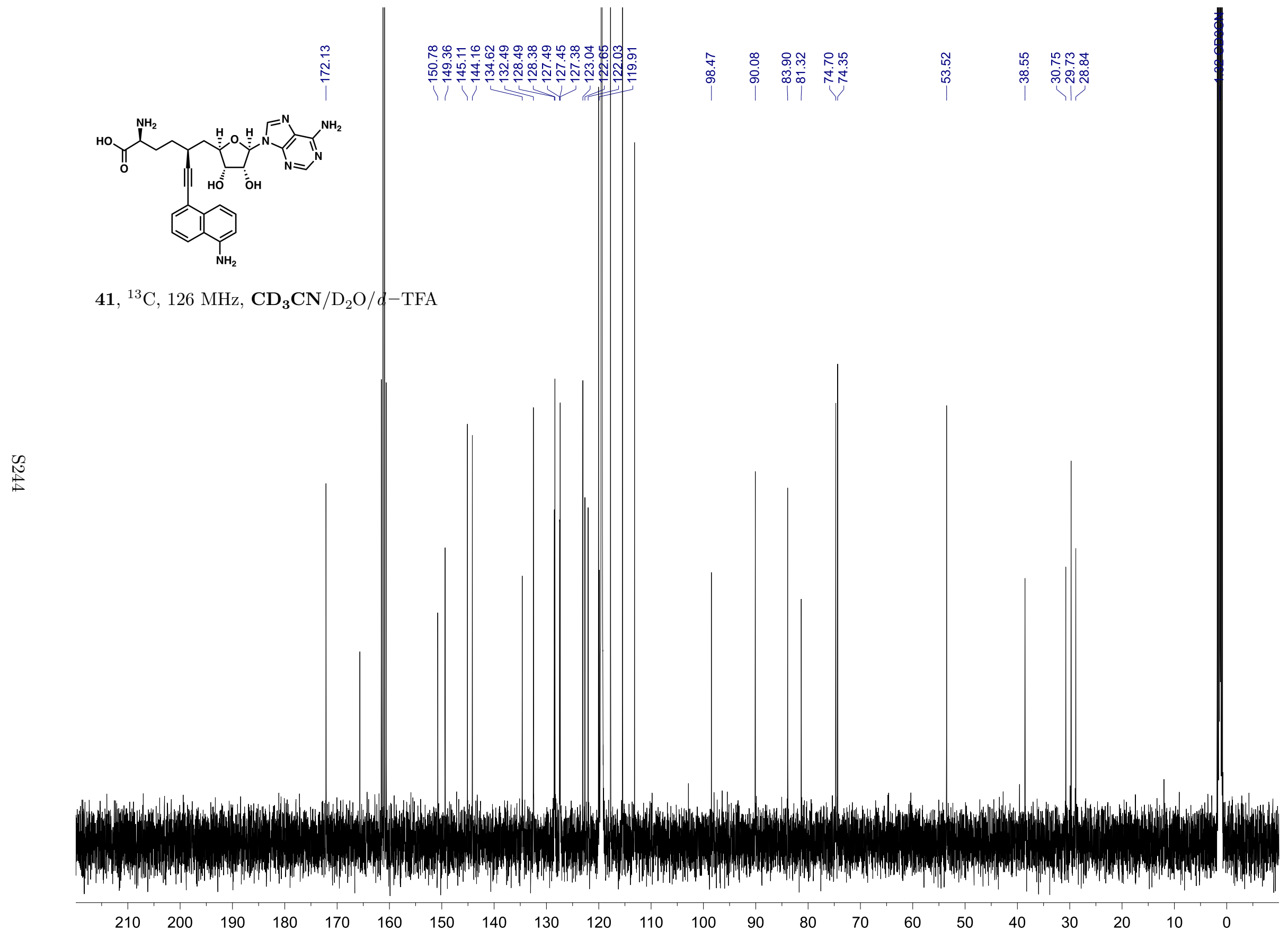

